\title{
Mechanisms of the intracellular localization of the SUMO-activating enzyme Aos1/Uba2
}

\author{
Dissertation \\ for the award of the degree \\ „Doctor rerum naturalium“ \\ Division of Mathmatics and Natural Sciences \\ of the Georg-August-Universität Göttingen
}

\author{
submitted by \\ Marie Christine Moutty \\ from Heide
}

Göttingen 2010 
Member of the Thesis Committee (First Reviewer):

Prof. Dr. Frauke Melchior

Center for Molecular Biology (ZMBH)

University of Heidelberg

Member of the Thesis Committee (Second Reviewer):

Prof. Dr. Ralf Ficner

Institute for Microbiology and Genetics, Department of Molecular Structural Biology

University of Göttingen

Member of the Thesis Committee:

Prof. Dr. Detlef Doenecke

Institute for Biochemistry and Molecular Cell Biology, Department of Molecular Biology University of Göttingen

Date of the oral examination: 04.05 .2010 


\section{Affidavit:}

I herewith declare, that this thesis has been written independently and with no other sources and aids than explicitly quoted.

Marie Christine Moutty 


\section{CONTENT}

$\begin{array}{ll}\text { ABSTRACT } & 5\end{array}$

INTRODUCTION 6

1. Posttranslational protein modification 6

1.1. Ubiquitin and ubiquitin-like modifiers $\quad 6$

1.2. The SUMO family 8

2. Molecular mechanisms of SUMOylation 9

2.1. Activation of SUMO 9

2.2. Conjugation to SUMO targets 12

2.3. Deconjugation of SUMOylated targets 13

3. Functions of SUMO modification 14

3.1. SUMO and transcriptional regulation 14

3.2. SUMO and the maintenance of chromosome stability 16

3.3. SUMO and nucleocytoplasmic transport 16

4. Nucleocytoplasmic transport $\quad 17$

4.1. Import 19

4.2. Export 20

5. Localization of SUMO enzymes 20

6. Aim of this work $\quad 23$

MATERIAL AND METHODS $\quad 24$

$\begin{array}{ll}\text { Material } & 24\end{array}$

1. Chemicals, reagents and enzymes 24

2. Reaction kits 25

3. Consumables 25

4. Buffers, media and stock solutions 26

5. Cell lines $\quad 29$

6. Oligonucleotides 29

7. Vectors and plasmids 31

8. Recombinant proteins 35 
9. Antibodies 36

10. Technical equipment and software 37

Methods $\quad 39$

1. Molecular biology methods $\quad 39$

1.1. Preparation of chemical competent bacteria $\quad 39$

1.2. Transformation of competent bacteria $\quad 39$

1.3. Plasmid DNA purification $\quad 39$

1.4. Measurement of DNA concentration 40

1.5. Agarose gel electrophoresis $\quad 40$

1.6. Isolation of DNA from agarose gels $\quad 40$

1.7. Restriction of DNA by endonucleases $\quad 40$

1.8. Ligation of DNA fragments $\quad 41$

1.9. Sequencing of DNA 41

1.10. Polymerase chain reaction (PCR) 42

1.11. Site directed mutagenesis 42

2. Biochemical methods 43

2.1. Measurement of protein concentration 43

2.2. SDS PAGE and detection of proteins 43

2.3. Protein precipitation with TCA 45

2.4. Expression and purification of recombinant proteins 45

2.5. Interaction experiments 49

2.6. Cell fractionation 50

2.7. In vitro SUMOylation reaction 52

2.8. Affinity purification of polyclonal antibodies 53

3. Cell biology methods for mammalian cells 54

3.1. Cultivation of adherent and suspension cells 54

3.2. Transient transfection 55

3.3. Fluorescence based detection of intracellular proteins 55

3.4. In vitro import assay 56

3.5. Microinjection into adherent HeLa cells 57

3.6. Analysis of nucleocytoplasmic shuttling 58

4. Yeast methods $\quad 59$

4.1. Culture and storage of yeast strains $\quad 59$

4.2. Preparation and transformation of competent yeast cells 60 
4.3. Processing of positive yeast transformants 61

4.4. Generation of yeast UBA2 shuffle strains 61

4.5. Phenotypic analysis of UBA2 shuffle strains 62

RESULTS 65

1. Generation and characterization of SUMO E1 variants 65

1.1. Reconstitution of $E 1$ complex from singly His-tagged subunits 65

1.1. Generation of fluorescently labeled E1 complex 66

1.2. Reconstituted His-Aos1/Uba2-His complex exhibits comparable specific activity to co-purified His-Aos1/Uba2 67

2. Characterization of the nuclear import of SUMO E1 68

2.1. Aos1 and Uba2 contain distinct functional NLSs 68

2.2. Importin $\beta$ binds via importin a to both Aos1 and Uba2 71

2.3. Importin $\alpha / \beta$ mediates import of Aos 1 and Uba2 in vitro and in cells $\quad 73$

2.4. Pre-assembled E1 complex can be imported into the nucleus 75

2.5. The NLS of Uba2 mediates $\mathrm{E} 1$ interaction with and import by importin $\alpha / \beta$ in vitro $\quad 77$

2.6. The SUMO E1 complex in cells is mainly imported by importin $\alpha / \beta \quad 79$

3. Analysis of the cytoplasmic E1 pool 80

3.1. Endogenous Aos1/Uba2 predominantly localizes to the nucleus of HeLa cells

3.2. SUMO E1 is found in cytosolic fractions of HeLa cells 82

3.3. Distribution of E1 in neuronal cells is analogous to HeLa cells 84

3.4. No indication for active export of the SUMO E1 86

5. Analysis of the effects of mislocalized SUMO E1 in yeast 92

5.1. Human Uba2 can not substitute for deletion of yeast Uba2 in UBA2 shuffle strains

5.2. Predominantly nuclear localization of Uba2 is not essential for the viabilty of yeast

5.3. Cytoplasmic localization of Uba2 in yeast does not significantly alter the SUMOylation pattern

1. Aos1 contains a c-Myc like NLS 100

2. Nuclear E1 is generated in two ways 101 
3. How is the cytoplasmic E1 pool generated? 103

4. Is the E1's intracellular distribution subjected to regulation? 105

5. Can the localization of Aos1 and Uba2 be seperately regulated? 106

6. Is cytoplasmic E1 a prerequisite for cytoplasmic SUMOylation? 107

$\begin{array}{ll}\text { 7. Perspectives } & 110\end{array}$

REFERENCES 111

SUPPLEMENTARY MATERIAL 132

$\begin{array}{ll}\text { ABBREVIATIONS } & 133\end{array}$

$\begin{array}{ll}\text { ACKNOWLEDGEMENTS } & 137\end{array}$

$\begin{array}{ll}\text { CURRICULUM VITAE } & 138\end{array}$ 


\section{ABSTRACT}

Dynamic posttranslational modification with ubiquitin related proteins of the SUMO (small ubiquitin-related modifier) family is an important cellular mechanism to alter the activity, abundance or localization of proteins. Reversible covalent attachment of SUMO to target proteins requires the catalytic activities of an E1-activating enzyme, an E2-conjugating enzyme, one of several E3 ligases as well as specific isopeptidases. Consistant with the existence of nuclear and cytoplasmic SUMO substrates, the components of the enzymatic machinery are also found in both compartments. This raises the interesting question how these pools are generated.

The presented work aimed to identify the mechanisms underlying the intracellular localization of the SUMO E1 complex. Since both subunits, Aos1 and Uba2, are predominantly localized in the nucleus, I initially determined the generation of the nuclear pool. I demonstrated that nuclear import can occur in two ways: Aos1 and Uba2 can be imported independently by distinct nuclear localization signals (NLSs), and the assembled complex can be imported by the NLS of Uba2. In both cases the import is mediated by the receptor importin $\beta$ and the adaptor protein importin $\alpha$.

Functional studies concerning the generation of the minor cytoplasmic pool of Aos1/Uba2 indicated that the proteins are not actively exported from the nucleus into the cytoplasm. Since the nuclear and cytoplasmic pools of E1 are not subjected to frequent exchange and the cytoplasmic fraction of $\mathrm{E} 1$ is very small I reasoned that the SUMO activating acitvity in the cytoplasm would be constantly low. This raised the question whether the E1's intracellular localization is at all of general importance for SUMOylation in different cellular compartments.

With yeast UBA2 shuffle strains, in which the endogenous UBA2 gene is deleted and cells are kept alive by a removable exogenous copy, I studied the ability of cytoplasmic E1 to substitute for endogenous E1. Surprisingly, deletion of endogenous E1 can be rescued both by exogenous cytoplasmic or nuclear E1. Yeast strains with either cytoplasmic or nuclear E1 exhibit similar modification patterns, indicating that the localization of E1 might not determine compartment specific SUMOylation. These findings suggest that thioester charged Ubc9 may shuttle to and allow efficient SUMOylation both in the nuclear and cytoplasmic compartment. 


\section{INTRODUCTION}

\section{Posttranslational protein modification}

Posttranslational modifications of proteins have crucial roles in biological systems since they participate in virtually all cellular processes. The enzymatic covalent modification of amino acid side chains within target proteins can affect protein function, activity, abundance or localization. Depending on the type of modification a distinction is drawn between the attachment of proteins, e.g. ubiquitylation or ubiquitin-like modifications, versus organic and anorganic molecules, for example acetylation, methylation, phosphorylation and glycosylation (reviewed in Walsh et al. 2005).

\subsection{Ubiquitin and ubiquitin-like modifiers}

Ubiquitin is the first discovered and best-characterized representative of a whole family of modification pathways - the ubiquitin-like (UBL) modifications. To date, 9 phylogenetic classes with at total of 17 human UBLs have been described, amongst them FAT10, ISG15, URM1, UFM1, ATG12 and the well-studied NEDD8 and SUMO (small ubiquitin-related modifier protein) pathways. All UBLs share the conserved ubiquitin or $\beta$-grasp fold exemplified in Fig. 1 and are attached to other proteins by similar pathways (Hochstrasser 2000; Welchman et al. 2005; Kerscher et al. 2006). Most UBLs are expressed as inactive precursor proteins and therefore have to be matured by C-terminal hydrolases. The mature modifier usually contains two glycine residues at the C-terminus (Amerik et al. 2004; Love et al. 2007). Despite these similarities each UBL system uses a discrete enzymatic cascade of E1 activating enzyme, E2 conjugating enzyme, and frequently E3 ligases, for modification. UBLspecific isopeptidases are required for maturation and for deconjugation of the according modification (for details see chapter INTRODUCTION - Molecular mechanism of SUMOylation).

The ubiquitin system comprises two E1 enzymes, Uba1 and Uba6, 37 E2 enzymes and more than $600 \mathrm{E} 3$ ligases (for references see Komander 2009). E3 ligases can be subdivided into two functionally different groups: HECT E3 ligases form a thioester with ubiquitin and thereby directly participate in the transfer reaction. In contrast, RING 

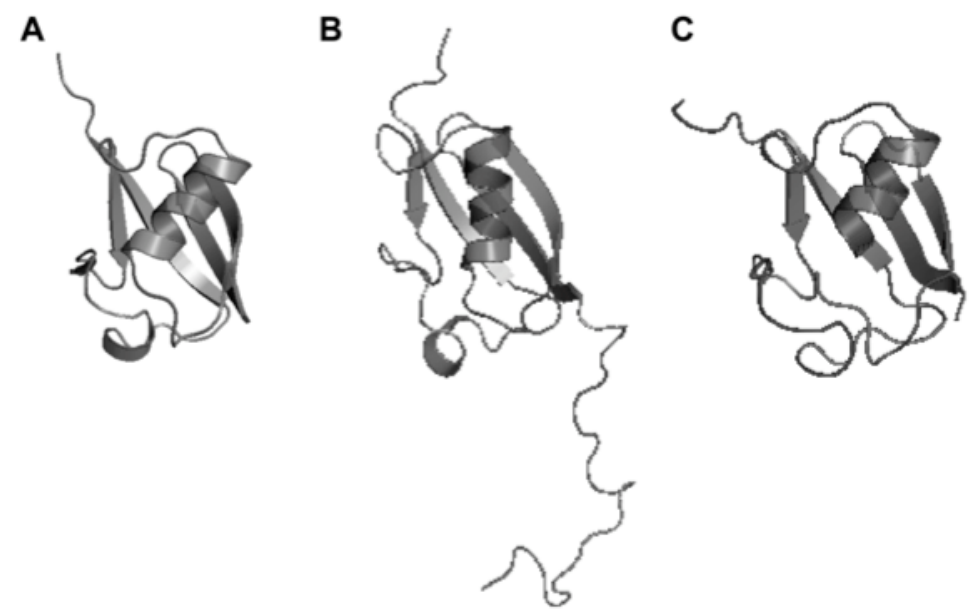

Figure 1: Conserved $\beta$-grasp fold of ubiquitin and UBLs. Cartoon of the structures of mature human ubiquitin (VijayKumar et al. 1987) (A), SUMO1 (Bayer et al. 1998) (B) and NEDD8 (Whitby et al. 1998) (C). Structures were oriented with the helix in front of the $\beta$-sheets and the C-terminal GlyGly-motifs on top. Files were obtained from the Protein Data Bank (PDB) and images were generated with PyMOL v0.99 (DeLano Scientific LLC).

finger E3 ligases facilitate the conjugation reaction by serving as bridging factors for the interaction between E2 enzymes and the target proteins (Pickart 2001). The ubiquitin system modifies thousands of substrates and is thereby involved in most, it not all, biological processes. Depending on the target protein and the set of enzymes used for modification, ubiquitylation can result in mono-, multiple mono- or polyubiquitylation, each of which exerts different effects on the same target. Monoubiquitylation is involved in many different intracellular processes, e.g. endocytosis, endosomal sorting, histone regulation, DNA repair and nuclear export (Haglund et al. 2003; Hicke et al. 2003; Mosesson et al. 2006), whereas multiple mono-ubiquitylation primarily participates in endocytosis (Haglund et al. 2003). Due to the presence of 7 internal lysine-residues targets can be modified with polyubiquitin chains (reviewed in Ikeda et al. 2008; Komander 2009). Well-known examples for polyubiquitylation are lysine48-linked chains that target substrates for proteasomal degradation (Hershko et al. 1998; Thrower et al. 2000; reviewed in Finley 2009) and lysine63-linked chains that are involved in endocytosis (Geetha et al. 2005; Duncan et al. 2006), DNA-damage repair (reviewed in Moldovan et al. 2007; Panier et al. 2009), and signal transduction (reviewed in Skaug et al. 2009). The biological signficance of other polyubiquitin chains have been less well investigated: While lysine11-linked chains have been reported to serve as proteasomal degradation signal (Baboshina et al. 1996; Xu et al. 2009) and to play a role in cell cycle of mammalian cells (Jin et al. 2008), the physiologal roles of lysine6, 27, 29, 33 linkages are largely unknown. To complicate matters, recent studies report the formation of mixed and branched ubiquitin linkages (Ben-Saadon et al. 2006; Kim et al. 2007; Kim et al. 2009). However the in vivo abundance and relevance of these chains requires further analysis. 


\subsection{The SUMO family}

Next to ubiquitin, the SUMO family is the best-characterized representative of UBL modifiers. SUMOylation has been found to be an essential process in many organisms (Johnson et al. 1997; Fraser et al. 2000; Nacerddine et al. 2005; Saracco et al. 2007) and SUMO proteins are ubiquitously expressed throughout the eukaryotic kingdom. Some organisms including $S$. cerevisiae, C. elegans and $D$. melanogaster have only one SUMO gene, whereas plants and vertebrates posses several SUMO genes. The human genome encodes for four SUMO proteins (Melchior 2000; Guo et al. 2004). Three of these paralogs, SUMO1 (also known as human Smt3c, PIC1, GMP1, sentrin and Ubl1), SUMO2 (also known as Smt3a and Sentrin3) and SUMO3 (also known as Smt3b and Sentrin2), are ubiquitously expressed in vertebrates (Guo et al. 2004). The mature forms of SUMO2 and SUMO3 are $97 \%$ identical, and can therefore functionally not be differentiated (Saitoh et al. 2000; Tatham et al. 2001). Consequently they are designated as the SUMO2/3 subfamily. SUMO2/3 and SUMO1 significantly differ in their primary sequences and are only $47 \%$ identical. Furthermore, the SUMO paralogs have different cellular functions as they are attached to different target proteins (Saitoh et al. 2000; Vertegaal et al. 2006). Whether the fourth paralog SUMO4 is also covalently attached to target proteins or acts via non-covalent mechanisms still remains to be completely clarified (Owerbach et al. 2005; Wei et al. 2008).

SUMOylation of target proteins predominantly results in conjugation of monomeric SUMO proteins. However, like ubiquitin, SUMOs can also form multimeric chains (reviewed in Ulrich 2008; Vertegaal 2010). While recent mass spectrometry studies give direct in vivo evidence for SUMO2/3 chains, attachment of SUMO1 on multimeric SUMO2/3 is suggested to limit chain extension (Matic et al. 2008; Hsiao et al. 2009), In contrast to the ubiquitin system, the enzymatic SUMOylation machinery is much less complex. To date only a single E1, human Aos1/Uba2, a single E2, human Ubc9, about ten E3 ligases and six SUMO-specific isopeptidases have been identified (reviewed in Geiss-Friedlander et al. 2007). 


\section{Molecular mechanisms of SUMOylation}

The capability of the SUMO system to modify and de-modify target proteins makes SUMOylation a reversible and highly dynamic posttranslational modification. The underlying molecular mechanisms resemble ubiquitination and other ubiquitin-like modifications, with an enzymatic cascade of E1, E2 and E3 causing conjugation of SUMO and isopeptidases catalyzing the de-conjugation (Fig.2). However, the set of enzymes involved in SUMOylation is distinct from the enzymes of other UBL systems (reviewed in Johnson 2004; Hay 2005; Geiss-Friedlander et al. 2007).

\subsection{Activation of SUMO}

SUMO proteins are translated as precursors and have to be processed by C-terminal hydrolases (SUMO specific cysteine proteases) prior to activation. Mature SUMO ends with the characteristic GlyGly-motif that is necessary for the attachment to target proteins. In the first step of the E1-E2-E3 cascade mature SUMO is activated by the SUMO specific E1 activating enzyme. The SUMO E1 consists of two subunits, Aos1

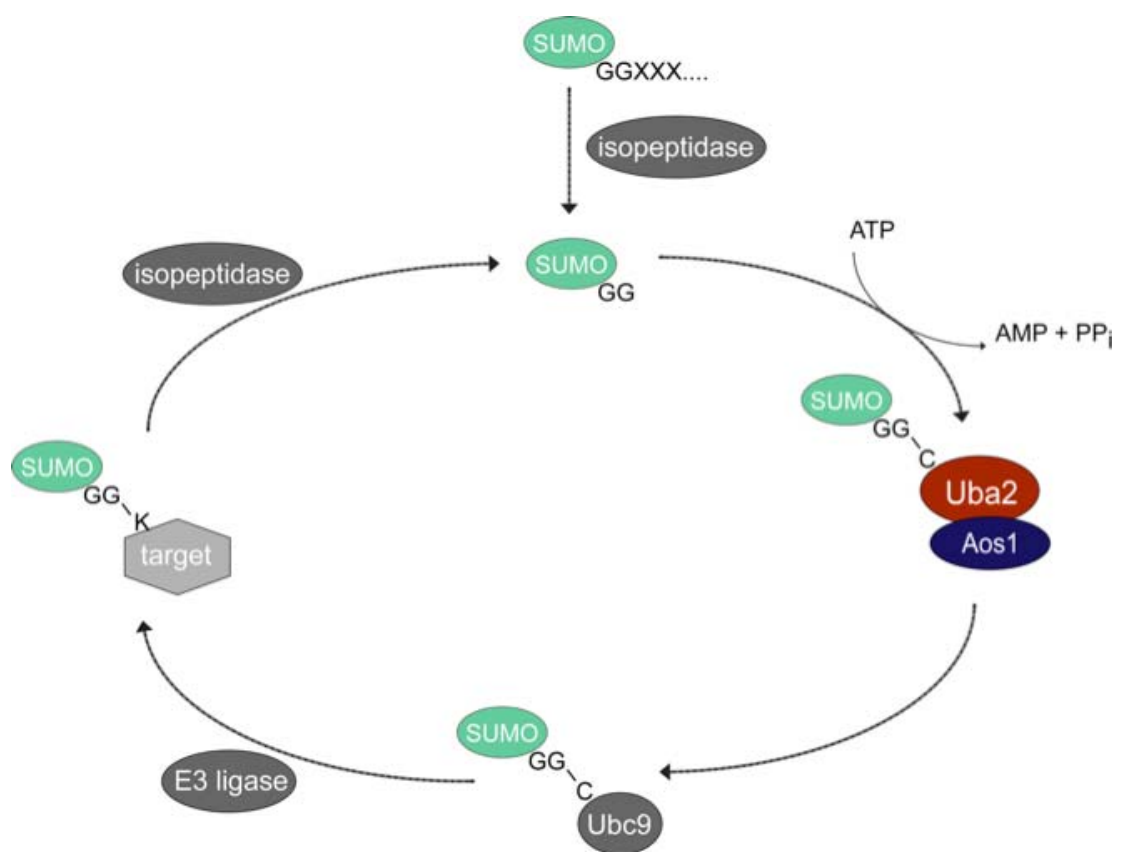

Figure 2: The enzymatic cascade of SUMOylation. Maturation of the SUMO precursor by hydrolases results in mature SUMO with a double glycin motif at the C-terminal end. The E1 activating enzyme, heterodimeric Aos $1 / \mathrm{Uba} 2$ complex, activates the C-terminal carboxy group of SUMO by forming a high-energy thioester bond with the E1's active site cysteine residue. Activated SUMO is then, in a transesterification reaction, transferred to a cysteine in the E2 conjugating enzyme Ubc9. Assisted by SUMO E3 ligases, Ubc9 conjugates SUMO to a variety of target proteins. The resulting isopeptide bond is stable, wherefore desumoylation of the targets requires the activity of specific isopeptidases. 


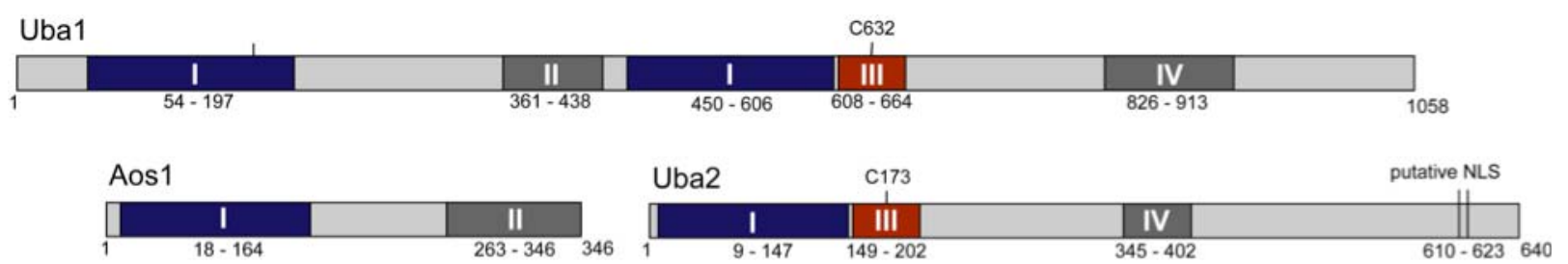

Figure 3: Schematic representation of homologous domains in the primary sequences of Ub-like E1 enzymes. Domains of high sequence similarity in the human ubiquitin E1 Uba1 and the SUMO E1 Aos1/Uba2 are highlighted. Domain I (blue) includes a potential nucleotide-binding motif and is found in a number of proteins unrelated to ubiquitin-like activating enzymes. Domain III (red) contains the active site cysteine of E1 enzymes that forms a thioester bond with the C-terminal carboxy-group of mature Ub-like proteins. The domains II and IV (dark grey) are found in several other proteins than E1 enzymes, but their function is so far unknown. Domains shown correspond to those described by Johnson et al. (Johnson et al. 1997). A putative NLS in Uba2 was described previously for the yeast homolog of Uba2 (Dohmen et al. 1995).

(also known as Sae1 or ULE1A) and Uba2 (also known as Sae2 or ULE1B) (Johnson et al. 1997; Desterro et al. 1999). The sequence of the SUMO E1 is highly similar to the ubiquitin E1 (Uba1). While Aos1 resembles the N-terminal half of Uba1, the second E1 subunit Uba2 resembles the C-terminal half (Fig. 3). Aos1 and Uba2 both contain a conserved nucleotide binding motif, GxGxxG (Wierenga et al. 1983) in domain I and a conserved region of so far undetermined function (domains II and IV) (Johnson et al. 1997; Desterro et al. 1999). The active cysteine, Cys-173 in Uba2, is located in an active-site consensus sequence $\mathrm{KxxPzCTxxxxP}$ ( $\mathrm{z}$ is an apolar residue) (Hatfield et al. 1992) within the conserved domain III. A striking difference in the primary sequences of Uba1 and Aos $1 / \mathrm{Uba} 2$ is the C-terminal region of Uba2, which is absent from the sequence of Uba1. This domain contains a putative consensus nuclear localization signal (NLS) and has been reported to be important for the nuclear localization of Uba2 (Dohmen et al. 1995; Desterro et al. 1999).

Similarities of Uba1 and Aos1/Uba2 are not only found in the primary sequences but also in the tertiary domain structure of the enzymes (Fig. 4). Comparison of the structures reveals similarities in the presence and position of the adenylation, the UbL (ubiquitin-like domain) and the catalytic cysteine domain (Lois et al. 2005). Interaction of Aos1 and Uba2 occurs via a relatively small surface in the adenylation domain of Uba2. While the catalytic cysteine domain harbours the active-site Cys173 that forms the thioester with SUMO, the UbL domain has an essential role in the recruitment of the E2 enzyme. 
A

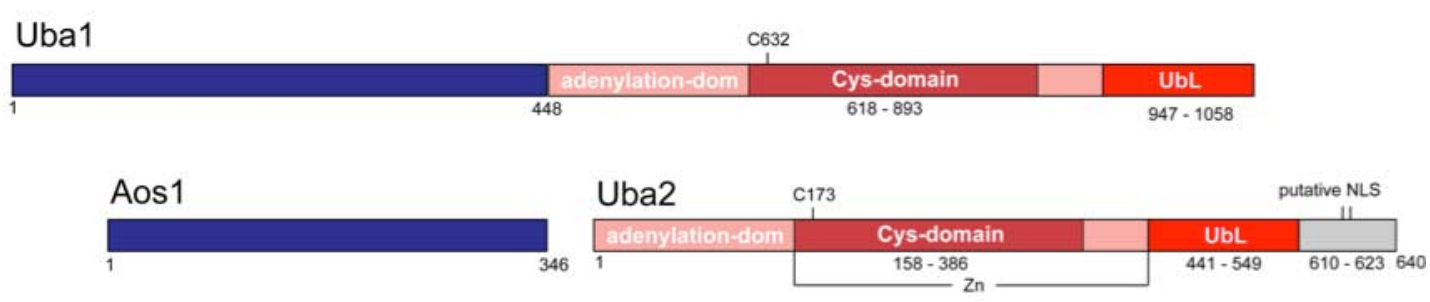

B

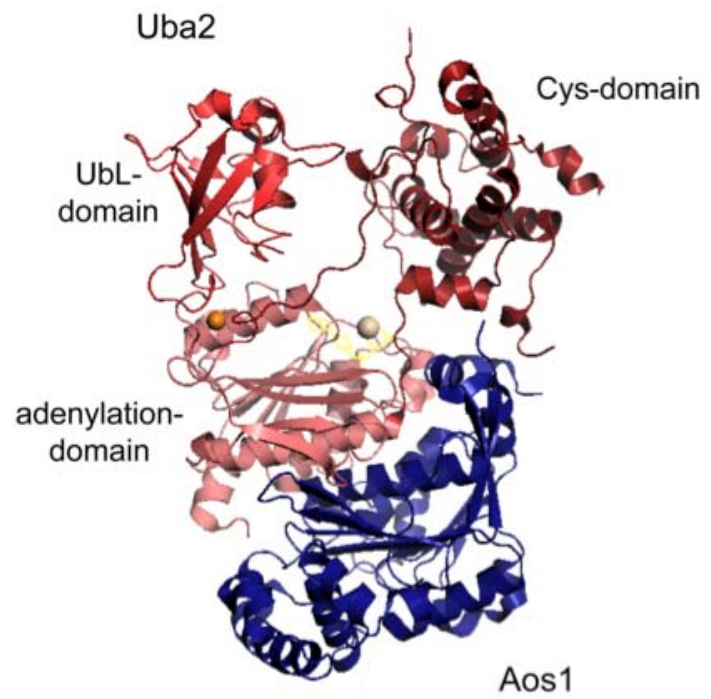

Figure 4: The tertiary domain structure of Aos/Uba2. (A) Schematic presentation of the structural domains of Uba1, Aos1 and Uba2. Aos1 is shown in blue and Uba2 in different shades of red: adenylation domain (faint red), catalytic cysteine domain (medium red), Ub-like domain (UbL) (intensive red) and the Uba2 Cterminus (grey). (B) Cartoon of the tertiary structure of Aos1/Uba2, Zn (orange ball), Mg (salmon ball) and ATP (yellow as stick diagram) (Lois et al. 2005). The colour code for Aos1/Uba2 corresponds to (A). The C-terminus of Uba2 (grey) was not present in the electron density map and is not contained in the structure. Files were obtained from the Protein Data Bank (PDB) and images were generated with PyMOL v0.99 (DeLano Scientific LLC).

Analogous to activation of ubiquitin, the ATP-dependent activation of SUMO catalyzed by human Aos1/Uba2 involves two distinct steps. First, the C-terminal carboxyl group of SUMO attacks the $\alpha$-phosphate of the ATP, forming a SUMO adenylate and releasing pyrophosphate. Then, the catalytic cysteine in Uba2 attacks the adenylate whereby a high-energy thioester bond between E1 and SUMO is formed and AMP is released (Johnson et al. 1997).

These events require dramatic rearrangements in the $E 1$ enzyme: While adenylation of SUMO is performed in an opened conformation, thioester-bond formation with Uba2 occurs in a closed conformation (Olsen et al. 2010). The structural changes from the opened to the closed state include a 130 degree rotation of the whole Cys-domain of Uba2 towards the adenylation site of the E1.

To complicate matters, activation of ubiquitin by Uba1 has been shown to involve asymmetric double loading of the E1 with two ubiquitin molecules (Haas et al. 1982; Haas et al. 1982; Schulman et al. 2009). Since a similar mechanism was found for the NEDD8 E1 complex NAE1/UBA3 (Bohnsack et al. 2003; Walden et al. 2003; Huang et al. 2007) it is very likely that a related mechanism is involved in the activation of 
SUMO by Aos1/Uba2. Thus, after thioester-bond formation, SUMO transfer from the $\mathrm{E} 1$ to the E2 is probably accompanied by the binding of a second SUMO molecule.

\subsection{Conjugation to SUMO targets}

After activation by the E1 enzyme SUMO is transferred in a transesterification reaction to the catalytic cysteine of the SUMO E2 enzyme Ubc9 (Fig. 2). This transfer reaction results in a thioester linkage between the thiol group of Ubc9's active cysteine and the C-terminal carboxy group of SUMO (Desterro et al. 1997; Johnson et al. 1997; Lee et al. 1998). In contrast to the ubiquitin system for which more than 20 E2 enzymes have been described, only a single SUMO E2 is known.

In the last step of the SUMOylation cascade SUMO is transferred to a target protein, by formation of an isopeptide bond between SUMOs C-terminal carboxy group and the $\varepsilon$-amino group of a lysine residue in the target protein. This step is mostly performed by the concerted action of the E2 enzyme and a SUMO E3 ligase that facilitates the conjugation.

Unlike HECT ubiquitin E3 ligases, which directly participate in the ubiquitin-conjugation by forming a thioester with ubiquitin, all known SUMO E3 ligases promote the direct transfer from the E2 enzyme to the target. Three types of SUMO ligases have been described in some detail (reviewed in Hay 2005; Geiss-Friedlander et al. 2007): SPRING ligases (Hochstrasser 2001), RanBP2 (Ran binding protein 2) (Pichler et al. 2002) and Pc2 (polycomb group protein 2) (Kagey et al. 2003).

SP-RING (Siz/PIAS-RING) ligases are the SUMO specific counterparts of the RING type ligases from the ubiquitin system. By direct interaction with thioester-charged Ubc9 and a target protein they position the SUMO-loaded E2 in a favorable position for the transfer of SUMO. The group of SP-RING ligases is composed of MMS21 (also known as NSE2) (Andrews et al. 2005; Potts et al. 2005; Zhao et al. 2005,) the meiosis specific yeast protein Zip3 (Cheng et al. 2006) and the PIAS family proteins (protein inhibitors of activated STAT). Recent chrystallographic analyis of the yeast PIAS-homolog Siz1 revealed that the central zinc-containing RING-like SP-RING domain and the SP-CTD (드-terminal domain) are required for the activation of the SUMO-E2 thioester, whereas the conserved N-terminal PINIT domain is essential for redirecting SUMO-conjugation to the acceptor lysine in the substrate (Takahashi et al. 2005; Yunus et al. 2009). Two PIAS family members, Siz1 and Siz2, have been described in S. cerevisiae and five in mammals, PIAS1, PIAS3, PIAS $\alpha$, PIASx $\beta$ and 
PIASy (Hochstrasser 2001; Jackson 2001). While the commonly shared SP-RING motif is essential for interaction with Ubc9, regions $\mathrm{N}$ - and C-terminal of the SP-RING motif mediate substrate recognition (Hochstrasser 2001).

In contrast to the family of SP-RING ligases, the SUMO specific ligases RanBP2 and Pc2 are unrelated to the ubiquitin E3s. The minimal catalytic domain of the nuclear pore protein RanBP2 is natively unfolded and assumes its structure upon folding around Ubc9 (Pichler et al. 2002; Pichler et al. 2004; Reverter et al. 2005). As RanBP2s minimal catalytic domain has not been detected to interact with any substrate, it is suggested to facilitate SUMO conjugation by positioning the Ubc9SUMO thioester for an optimal attack by the acceptor lysine residue in the modified target protein (Pichler et al. 2002; Tatham et al. 2005).

Pc2 is a member of the human polycomb group (PcG) proteins that form large multimeric complexes. It has been shown to stimulate SUMOylation of the transcriptional co-repressor CtBP (Kagey et al. 2003; Kagey et al. 2005). While the exact mechanisms of how Pc2 functions are not yet fully unterstood, a very recent study indicates that the SUMO interaction motifs of Pc2 play in important role in its E3 acitvity (Merrill et al. 2010).

The attachment of SUMO to target proteins typically involves specific lysine residues within the targets. Preferred regions of SUMOylation were defined as consensus sequence $\psi \operatorname{KxE}$ ( $\psi$ is a bulky hydrophobic residue) (Desterro et al. 1998; Melchior 2000). If the motif is accessible, Ubc9 can bind to it and subsequently transfer SUMO to the acceptor lysine in the consensus site of the target (Sampson et al. 2001; Bernier-Villamor et al. 2002). However, SUMOylation at consensus sites is not the only mechanism, as some targets are known to be modified at non-consensus lysines, e.g. PCNA (Hoege et al. 2002), E2-25K (Pichler et al. 2005) or USP25 (Meulmeester et al. 2008).

\subsection{Deconjugation of SUMOylated targets}

SUMO isopeptidases can remove SUMO from a modified target protein and thereby make SUMOylation a reversible and highly dynamic posttranslational modification. In addition to their isopeptidase activity, these enzymes exhibit a C-terminal hydrolase activity by which they can remove a short C-terminal peptide from the SUMO precursor protein generating mature SUMO (Fig. 2). By means of these two activities, SUMO isopeptidases are required for providing free conjugatable SUMO. 
All known SUMO isopeptidases are members of the family of Ulp cysteine proteases (ubiquitin-like modifier protease). Two SUMO-specific isopeptidases, Ulp1 and UIp2, have been identified in yeast ( $\mathrm{Li}$ et al. 1999; $\mathrm{Li}$ et al. 2000) and, to date, six mammalian Ulp homologs are known, SENP1, 2, 3, 5, 6 and 7 (sentrin-specific isopeptidase). The SENP isoforms differ from each other by their catalytic activities in maturation and deconjugation of SUMO, in their specificity towards the SUMO paralogs (Di Bacco et al. 2006; Gong et al. 2006) and also in their intracellular localization (Mukhopadhyay et al. 2007) (for details see chapter INTRODUCTION Localization of SUMO targets and enzymes). However, regarding the large number of ubiquitin specific proteases, it can be speculated that the list of identified SUMO isopeptidases might not yet be complete.

\section{Functions of SUMO modification}

The reversible and highly dynamic modification of target proteins with the small modifier SUMO can affect the characteristics of the target protein in three mechanistically different ways (Fig. 5): Attachment of the SUMO moiety can prevent or enable interactions with other macromolecules (proteins, DNA or RNA) or it can induce intramolecular structural rearrangements. Depending on the target and on the site at which the specific substrate is modified, SuMOylation can alter its function by influencing its activity, stability or localization in the cell. Due to the large number of known SUMO targets, SUMOylation contributes to a large and still growing number of pathways including transcriptional regulation, maintenance of genome integrity, signal transduction and nucleocytoplasmic transport (reviewed in Geiss-Friedlander et al. 2007; Zhao 2007). In the following, the function of SUMOylation is exemplified for some selected pathways.

\subsection{SUMO and transcriptional regulation}

One of the first discovered and probably best-known roles of SUMO modification is the regulation of transcription. The huge number of transcriptional regulators that have been shown to be SUMOylated indicates the importance of SUMOylation in transcription. 


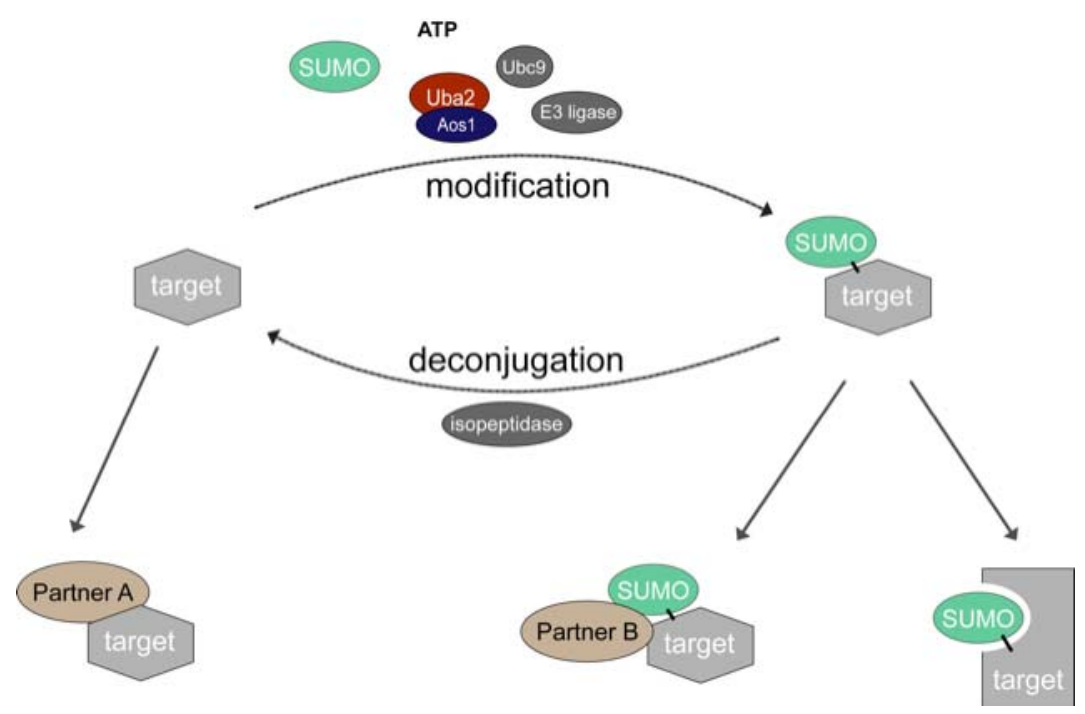

Figure 5: Molecular consequences of reversible SUMOylation: (A) SUMOylation can prevent the interaction of the target with partner $A$, only enabling the interaction in the absence of the SUMO modification. (B) SUMOylation can facilitate the interaction between the target and its partner B by providing an additional binding site for the interaction partner B. (C) SUMOylation can induce intramolecular conformational changes of the modified target protein. Adopted from (GeissFriedlander et al. 2007).

SUMOylation of transcription factors mostly causes transcriptional repression by inhibiting the activity of transcriptional activators (Gill 2005). This can occur by different mechanisms, e.g. inhibition of the DNA-binding capability (Anckar et al. 2006) or altered intracellular localization of transcription factors like LEF1 (Sachdev et al. 2001) and lipin1- $\alpha$ (Liu et al. 2009).

In addition, SUMO modification of transcription factors can lead to local formation of heterochromatin-like silenced DNA, as has been shown for the transcription factor Sp3 (Stielow et al. 2008). Sp3 SUMOylation recruits chromatin remodelling factors and histone methyltransferases leading to repressive modification of histones and attachment of heterochromatic proteins.

Modification with SUMO has in some cases also been shown to cause transcriptional activation. This is exemplified by the transcription factor Oct4 which has been reported to show increased DNA-binding upon SUMOylation (Wei et al. 2007) or the transcriptional repressor Tel whose association with DNA has been found to be impaired by its SUMOylation (Roukens et al. 2008). 


\subsection{SUMO and the maintenance of chromosome stability}

Repair mechanisms ensure the proper propagation of the cellular genome during several cycles of cell division. A very intriguing example for the role of SUMOylation in postreplication repair processes is the modification of PCNA (proliferating cell nuclear antigen) (reviewed in Watts 2006; Ulrich 2009). PCNA serves as a processivity clamp for replicative DNA polymerases, whose ubiquitylation upon DNA damage promotes the bypass of replication-blocking lesions and thereby leads to DNA damage tolerance (reviewed in Lehmann et al. 2007). In contrast, SUMOylation of PCNA has been shown to negatively affect the bypass of lesions (Stelter et al. 2003). SUMOylation of PCNA recruits the anti-recombinogenic DNA helicase Srs2 to the replication forks where the helicase prevents spontaneous recombination of the single-stranded DNA (Papouli et al. 2005; Pfander et al. 2005). Consequently, PCNA SUMOylation prevents increased recombination between sister chromatids (Robert et al. 2006).

Another example for SUMOylation influencing DNA repair is the base excision repair enzyme thymidine DNA glycosylase (TDG). SUMO modification reduces the affinity of TDG for DNA and thereby helps to release the enzyme from mismatch lesions after removal of $U$ or T (Hardeland et al. 2002; Baba et al. 2005; Steinacher et al. 2005; Fitzgerald et al. 2008).

\subsection{SUMO and nucleocytoplasmic transport}

SUMOylation has been implicated in the regulation of nuclear transport of cargo proteins. Interestingly, it has been shown by the laboratory of Frauke Melchior that the nuclear pore protein RanBP2 (Ran binding protein 2, also referred to as Nup358) contains SUMO E3 ligase activtiy (Pichler et al. 2002). RanBP2 is the main component of the cytoplasmic filaments of nuclear pores complexes (NPC) (Wilken et al. 1995; Wu et al. 1995; Yokoyama et al. 1995) and specifically interacts with the SUMOmodified form of RanGAP1, the first identified SUMO-substrate, which by itself is a soluble cytoplasmic protein (Matunis et al. 1996; Mahajan et al. 1997). In addition, the SUMO E2 enzyme Ubc9 has been shown to interact with the RanBP2/RanGAP1SUMO complex (Saitoh et al. 1997; Lee et al. 1998). Thereby SUMO modifying activity of Ubc9 and RanBP2 is combined with components of the nucleocytoplasmic tranport machinery, RanBP2 and RanGAP1. Furthermore, the isopeptidase SENP2 has been shown to localize to the nuclear site of the NPCs via interaction with the nucleoporin Nup153 (Hang et al. 2002; Zhang et al. 2002). These data indicate that SUMOylation 
and deSUMOylation can occur at the entry and exit sites of NPCs, pointing to an interesting role of dynamic SUMOylation in nucleocytoplasmic transport (Pichler et al. 2002).

Direct evidence for a role of SUMOylation in nuclear import has been obtained in $S$. cerevisiae. Mutants of the SUMO conjugation machinery have been shown to cause nuclear accumulation of the yeast importin $\alpha$-homolog Srp1 and thereby lead to inhibition of cNLS-dependent nuclear import (Stade et al. 2002).

In addition, some examples provide indirect indications for a role of SUMOylation in the nucleocytoplasmic distribution of proteins: On the one hand nuclear enrichment of SUMOylated forms has been described for targets like FAK (Kadare et al. 2003), caspase-7, -8 and procaspase-2 (Besnault-Mascard et al. 2005; Shirakura et al. 2005; Hayashi et al. 2006) and the centrosome-associated protein ninein (Cheng et al. 2006). On the other hand SUMOylation has been reported to induce nuclear export of Dictyostelium Mek1 Sobko et al. 2002 and to enhance the interaction between the bovine papilloma virus (BPV) E1 and its export receptor CRM1 (Rosas-Acosta et al. 2008). Although it is not always clear whether SUMOylation directly alters the transport process or anchors proteins in a compartment, the examples demonstrate a role of SUMOylation in the nucleocytoplasmic distribution of target proteins.

\section{Nucleocytoplasmic transport}

Millions of proteins and ribonucleoprotein particles have to enter and/or leave the nuclear compartment during the lifetime of a cell. Nuclear pore complexes (NPCs), anchored in the nuclear envelope, form the connection between the nucleus and the cytoplasm and enable a controlled exchange between these compartments. NPCs are huge complexes composed of several copies of about 30 different proteins, the nucleoporins (Cronshaw et al. 2002). Small molecules and proteins of a size below the threshold of approximately $50 \mathrm{kDa}$ can diffuse through NPCs between cytoplasm and nucleoplasm. However, transport cargoes have to be actively transported in and out of the nucleus. Specific transport receptors bind to signal sequences within the cargo protein and mediate the translocation through the nuclear pore by interacting with nucleoporins (Fig. 6). Depending on the direction of transport these transport signals are designated as nuclear import signals (NLSs) or nuclear export signals (NESs) (reviewed in Fried et al. 2003; Weis 2003). 
The direction of transport is defined by differential affinities of import or export receptor for their cargoes depending on the presence of RanGTP (Fig. 6). Import receptors bind their cargo only in the absence and release the cargo in the presence of RanGTP. In contrast, export receptors bind the cargo in the presence and release it in the absence of RanGTP. This system functions due to the compartment specific localization of Ran's key regulators, the guanine nucleotide exchange factor RCC1 and Ran's GTPase activating protein RanGAP1. While RCC1 is restricted to the nucleus and ensures a high concentration of RanGTP inside of the nucleus, RanGAP1 is localized in the cytoplasmic assuring that most cytoplasmic Ran is bound to GDP instead of GTP (for a collection of detailed reviews see Kehlenbach 2009).

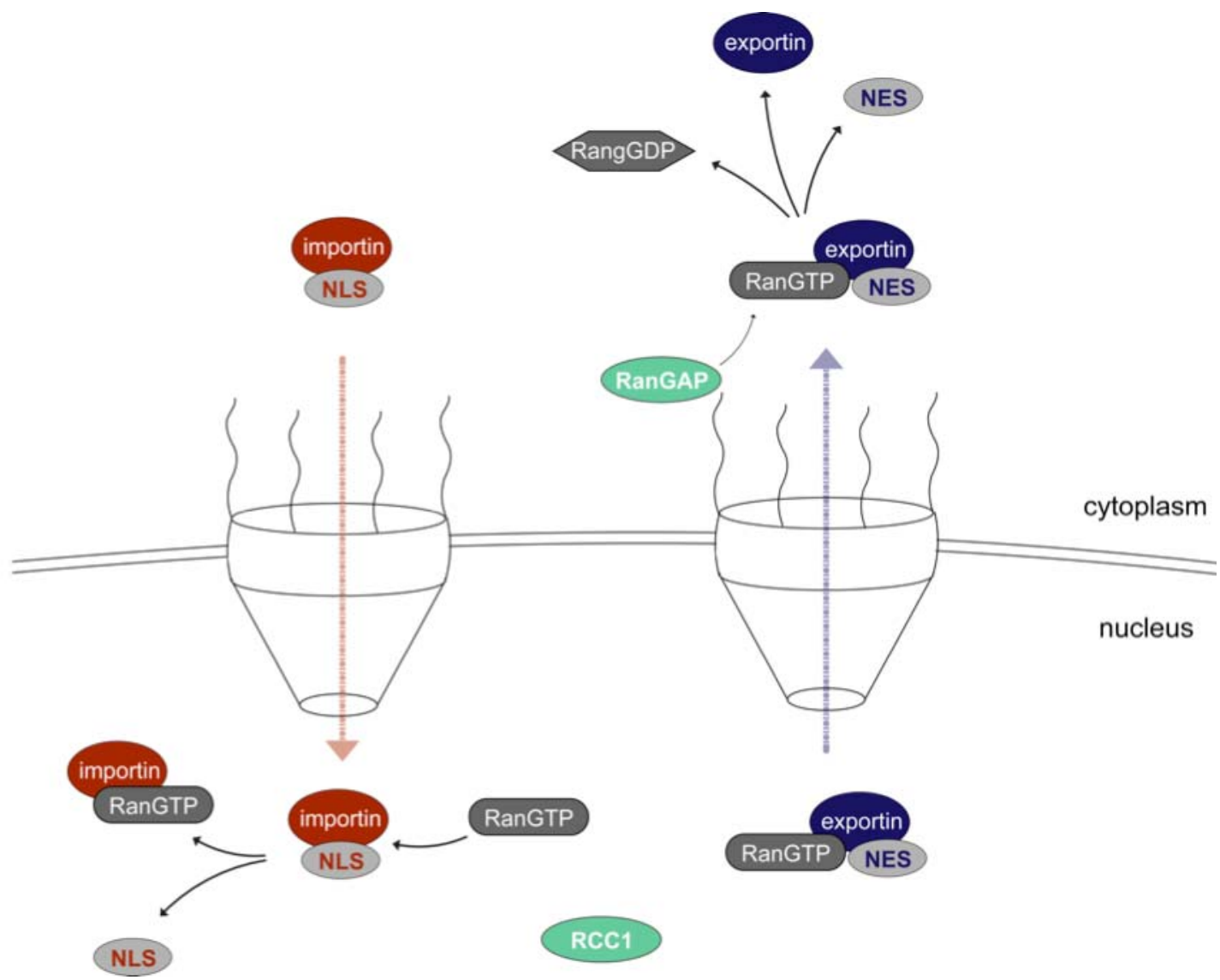

Figure 6: Simplified model of the mechanism of nucleocytoplasmic transport across the nuclear envelope. Schematic presentation of nuclear import (left) and export (right) depending on the status of Ran. The nuclear localization of the RanGEF (guanine nucleotide exchange factor) RCC1 and the cytoplasmic localized RanBP1, RanBP2 and RanGAP1 (GTPase activating enzyme) determine the compartment-specific formation or disassembly of import and export complexes. 


\subsection{Import}

Nuclear import begins in the cytoplasm, where import receptors bind to the NLS of cargo proteins. Most import receptors, amongst them transportin, importin $\beta$, importin 5,7 and 9 , belong to the importin $\beta$ family and vary in their preference for different NLSs. For example, transportins preferentially recognizes glycine-rich NLSs (M9sequences) present for example in hnRNPs (Pollard et al. 1996; Siomi et al. 1997) or RS domain containing SR proteins (Kataoka et al. 1999; Lai et al. 2001). Other family members directly bind to stretches of basic amino acids found within ribosomal proteins (Jakel et al. 1998), core histones (Mosammaparast et al. 2001; Muhlhausser et al. 2001; Mosammaparast et al. 2002) and many other proteins. However, the bestcharacterized import signals within cargo proteins are classical NLSs (cNLSs). Monopartite cNLS, such as the SV40 large T antigen NLS (Kalderon et al. 1984), consist of one cluster of basic amino acids, whereas bipartite cNLSs, first found in nucleoplasmin (Dingwall et al. 1982; Robbins et al. 1991), are composed of two basic clusters separated by approximately 10 random amino acids. Nuclear import via a cNLS involves an adaptor protein of the importin $\alpha$ family, which binds to the cargo NLS and simultaneously, by its IBB domain (importin $\beta$ binding domain), to the import receptor importin $\beta$ via its IBB (importin $\beta$ binding) domain.

The typical import complex of cargo/importin $\alpha / \beta$ is formed in the cytoplasm in the absence of RanGTP (Fig. 6, left). After translocation through the NPC the import complex reaches the nucleoplasm where a high concentration of RanGTP is present. RanGTP interacts with importin $\beta$ whereby the importin $\alpha / \beta$ complex and consequently the whole transport complex disassembles and the cargo is released into the nucleoplasm (Rexach et al. 1995; Gorlich et al. 1996). Subsequently importin $\alpha$ and importin $\beta$ have to enter the cytoplasm to be available for another round of import. While importin $\beta /$ RanGTP directly translocates back to the cytoplasm, importin $\alpha$ interacts with its own specific export receptor CAS and enters the cytoplasm in form of importin $\alpha /$ CAS/RanGTP complex (Kutay et al. 1997; Hood et al. 1998; Kunzler et al. 1998). Finally, in of the cytoplasm, GTP is hydrolyzed to GDP and the receptors are released in the cytoplasm, available for another import cycle. 


\subsection{Export}

Nuclear export of most proteins is mediated by the export receptor CRM1 (chromosome region maintenance 1) (Fornerod et al. 1997; Stade et al. 1997; Hutten et al. 2007). CRM1 binds to leucine-rich NESs within export cargos, first described for the viral HIV-1 protein Rev (Fischer et al. 1995) and the cAMP-dependent proteinkinase inhibitor PKI (Wen et al. 1995). From these classical targets a consensus leucine-rich NES L- $\mathrm{X}_{2-3}-(\mathrm{L}, \mathrm{I}, \mathrm{V}, \mathrm{M}, \mathrm{F})-\mathrm{X}_{2-3}-\mathrm{L}-\mathrm{X}-(\mathrm{L}, \mathrm{I}, \mathrm{V})$ was deduced. In addtition CRM1, CAS (Kutay et al. 1997) and the exportins t, 1, 4, 6, 7 (Arts et al. 1998; Kutay et al. 1998; Lipowsky et al. 2000; Brownawell et al. 2002; Stuven et al. 2003; Lund et al. 2004) have been shown to mediate nuclear export. Furthermore, the receptors importin13 and transportin, primarily known for their function in nuclear import, have also been shown to mediate export of selected cargoes (Gallouzi et al. 2001; Mingot et al. 2001; Shamsher et al. 2002).

Inside the nucleus, in the presence of the GTP-bound form of Ran, CRM1 forms a trimeric export complex with the NES-containing cargo protein and RanGTP (Fig. 6, right). The CRM1/RanGTP/cargo complex translocates through the NPC into the cytoplasm. At the cytoplasmic side of the NPC, the action of RanGAP1 stimulates Ran GTP-hydrolysis and thereby causes the disassembly of the export complex and the release of the export cargo into the cytoplasm. The Ran molecule that has been exported by this action is subsequently re-imported by its own import receptor NTF2 (nuclear transport factor 2) (Ribbeck et al. 1998; Smith et al. 1998).

As nuclear import or export require the presence and the accessibility of a NLS or a NES, conformational changes or posttranslational modifications of the cargo protein can sterically hinder the receptor-cargo interaction, preventing nuclear transport. These mechanisms allow the specific regulation of nuclear import and export and can thereby determine the intracellular distribution of a particular target (Fabbro et al. 2003; Terry et al. 2007).

\section{Localization of SUMO enzymes}

SUMOylation affects hundreds of proteins at different intracellular localizations. Based on proteomics studies and on immunofluorescence analysis, the majority of SUMOylated proteins are found in the nuclear compartment. Amongst those are 
nuclear body proteins such as PML and SP100 (reviewed in Seeler et al. 2001), as well as many proteins involved in transcription (reviewed in Lyst et al. 2007; GarciaDominguez et al. 2009; Ouyang et al. 2009), chromatin remodelling (reviewed in Ouyang et al. 2009) and genome integrity (reviewed in Bergink et al. 2009). However, SUMOylated proteins are also found in the cytoplasmic compartment. Famous examples are mammalian RanGAP1 at the cytoplasmic side of the nuclear pore (Matunis et al. 1996; Mahajan et al. 1997), yeast septines located at the bud neck (Johnson et al. 2001; Takahashi et al. 2001; Takahashi et al. 2008), the ER-associated tyrosine phosphatase PTP1B (Dadke et al. 2007) and a number of proteins in the plasma-membrane, e.g. the voltage-gated potassium channel Kv1.5 (Benson et al. 2007), the kainate-receptor subunit GluR6 (Martin et al. 2007), the metatropic glutamate receptor mGluR8 (Tang et al. 2005), the glucose transporters GLUT1 and 4 (Giorgino et al. 2000) and the type I TGF- $\beta$ receptor ALK5 (Kang et al. 2008).

A prerequisite for the reversible modification of SUMO targets is the presence of the enzymatic SUMO machinery. As some of the identified substrates are restricted to the cytoplasm, the enzymes should also be present in that compartment. And indeed, although most of the enzymes are enriched in the nucleus, most components have also been found in the cytoplasm (Fig. 7).

Both subunits of the SUMO E1 Aos1/Uba2 have been shown to predominantly localize to the nucleoplasm of mammalian and yeast cells (Dohmen et al. 1995; Azuma et al. 2001), but have also been detected in mammalian cytosol using fractionation studies (Pichler et al. 2002; Bossis et al. 2006). Analysis of the intracellular localization of Uba2 during embryogenesis of $D$. melanogaster revealed changes of the distribution of Uba2 between nucleus and cytoplasm during embryogenesis (Donaghue et al. 2001; Shih et al. 2002).

A mostly nuclear localization has been reported for the E2 enzyme Ubc9 (Seufert et al. 1995), but fractions of the enzyme have also been detected in the cytoplasm and associated with cytoplasmic filaments of the NPC (Lee et al. 1998; Pichler et al. 2002; Zhang et al. 2002; Bossis et al. 2006). Notably, the cytoplasmic pools of E1 and E2 have been shown to be specifically regulated by reactive oxidative species (Bossis et al. 2006): Macrophage activation causes the production of $\mathrm{H}_{2} \mathrm{O}_{2}$ close to the plasmamembrane, which in turn causes an inactivating crosslink of cytoplasmic Uba2 and Ubc9. 
Members of the PIAS family of SUMO E3 ligases have been shown to be enriched in intranuclear dots, at least in part PML bodies, and additionally localize to the nucleoplasm and at low levels also to the cytoplasm (Sachdev et al. 2001; Kotaja et al. 2002; Miyauchi et al. 2002). However, the intracellular localization of the E3 ligases RanBP2 and Pc2 is restricted to a specific compartment, as RanBP2 is a component of the cytoplasmic filaments of the NPC (Wu et al. 1995; Yokoyama et al. 1995) and Pc2 localizes to intranuclear PcG bodies (Kagey et al. 2003; Roscic et al. 2006).

Finally, SUMO isopeptidases, necessary for the deconjugation and maturation of SUMO, are also found in both compartments. For example, S. cerevisiae Ulp1 and mammalian SENP2 are enriched at NPCs (Hang et al. 2002; Zhang et al. 2002; Li et al. 2003), SENP5 is enriched in the nucleolus (Nishida et al. 2000; Di Bacco et al. 2006) although a small fraction is found and required in the cytoplasm (Zunino et al. 2007), SENP1 can shuttle between nucleus and cytoplasm (Gong et al. 2000; Bailey et al. 2004) and SENP6 is found in both nucleus (Mukhopadhyay et al. 2006) and

nucleus cytoplasm
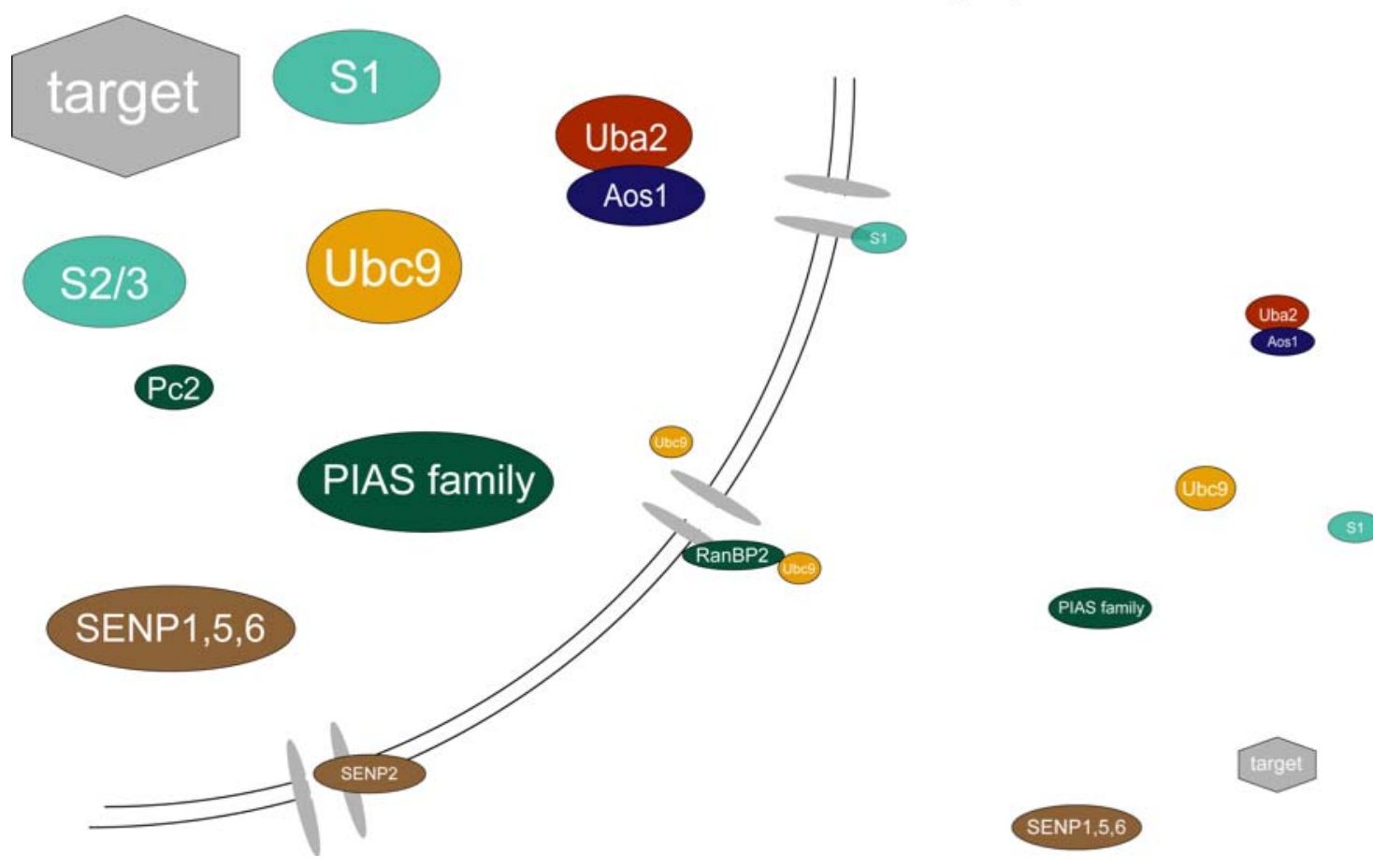

Figure 7: Schematic illustration of the estimated intracellular localization of the components of the SUMO system. Illustration of the distribution of SUMO proteins and components of the enzymatic machinery between nucleus and cytoplasm. The sizes of the icons rougly represent the relative amount of the enzymes, displaying the overall differences between the nuclear and cytoplasmic pools of the enzymes. Classes of enzymes are assigned to the following colourcode: the E1 enzyme Aos1/Uba2 in blue/red, the E2 Ubc9 in organge, E3 ligases in green and isopeptidases in brown. SUMO proteins are coloured turquoise and SUMO targets are shown in grey. 
cytoplasm (Kim et al. 2000).

In conclustion, members of all classes of SUMO enzymes are present in the nucleus and in the cytoplasm, which allows dynamic SUMOylation in both compartments. However, to date only little is known about the mechanisms generating these enzymatic pools, not to mention their regulation.

\section{Aim of this work}

The SUMO E1 enzyme Aos1/Uba2 is predominantly localized in the nuclei of cells, with small amounts also present in the cytoplasm. The aim of this work was to analyze the molecular mechanisms and significance of the intracellular distribution of the SUMO-activating enzyme.

Key questions to be addressed were if and how the individual subunits and/or the assembled complex are imported into the nucleus and if the E1 enzyme can shuttle between nucleus and cytoplasm. In addition, I aimed to address the question whether the intracellular localization of the E1 complex is critical for SUMOylation. 


\section{MATERIAL AND METHODS}

\section{Material}

\section{Chemicals, reagents and enzymes}

Standard chemicals and buffer substances were obtained from AppliChem, CARL ROTH, Serva, Sigma-Aldrich and Merck. The sources of special chemicals, reagents and enzymes are listed below:

$\left[\gamma^{32} \mathrm{P}\right] \mathrm{GTP}$

Amersham

Acrylamid (30 \%)

AppliChem

Aprotinin

Biomol

ATP

Sigma-Aldrich

BSA, fraction $V$

AppliChem

Creatinphosphate

Calbiochem

Creatinphosphate kinase

Calbiochem

Digitonin

Calbiochem

DMEM (high glucose)

Gibco

DNA marker $(1 \mathrm{~kb})$

Fermentas

dNTPs

Fermentas

sorbitol

Fluka

ECL

Pierce

FCS

Gibco

Ficoll 400

MP Biomedicals

Fluorescence Mounting Medium

DakoCytomation

GDP and GTP

Sigma-Aldrich

Hoechst 33258, Hoechst 33342

Sigma-Aldrich

IPTG

Fermentas

Leptomycin B (LMB)

present of Dr. M. Yoshida (Tokio, Japan)

Leupeptin

Biomol

Oligonucleotides

MWG; Operon; Sigma-Aldrich

OptiMEM

Invitrogen

Pepstatin

Biomol

Phusion polymerase

New England Biolabs 
PMSF

Sigma-Aldrich

Polyfect

Qiagen

Restriction enzymes

Fermentas, New England Biolabs

Rnase A

AppliChem

lodoacetamide

Sigma

Methyl methanesulfonate (MMS)

Fluka

Sodium fluoride (NaF)

Sigma

$\mathrm{N}$-Ethylmaleimide (NEM)

Sigma

Sequencing mix and buffer

Applied Biosystems

T4 DNA ligase

Fermentas

Trypane Blue

Fluka

Vent polymerase

New England Biolabs

Zymolyase 20T

MP Biomedicals

2. Reaction kits

NucleoBond $\AA$ PC 100, PC500

Macherey \& Nagel

NucleoSpin ${ }^{\circledR}$ RNAll

Macherey \& Nagel

NucleoSpin ${ }^{\circledR}$ Extract II

Macherey \& Nagel

QIAquick ${ }^{\circledR}$ Gel Extraction Kit

Qiagen

QIAquick ${ }^{\circledR}$ PCR Purification Kit

Qiagen

BCA Protein Assay kit

Pierce

BCA Protein Assay kit - Reducing Agent Compatible

Pierce

\section{Consumables}

Autoradiography films (Amersham HyperfilmMECL)

GE Healthcare

Cell culture consumables

Sarstedt, TPP

Centrifugal filter units

Millipore, Vivaspin

Cyanogen bromide-activated sepharose 4B

Sigma-Aldrich

Dialysis tubing Spectra-Por

Roth

Disposable plastic columns Bio-Spin, Poly-Prep, Econo-Pac

Bio-Rad

Filter paper 2MM Whatman

Whatman

Gloves (Rotiprotect-LATEX, -NITRIL) 
Microscope cover glasses (12 mm diameter, $18 \times 18 \mathrm{~mm}$ )

Marienfeld

Microscope slides (76x26x1 mm)

Marienfeld

ProBond ${ }^{\mathrm{TM}}$ Nickel-chelating resin

Invitrogen

PROTRAN nitrocellulose

Schleicher \& Schuell

Reaction tubes

Sarstedt, Eppendorf

Sterile filters and membranes $(0.22-0.45 \mu \mathrm{M})$

Millipore, Pall, Renner, Sartorius

\section{Buffers, media and stock solutions}

Buffers and stock solutions were prepared in deionized water and media were sterilized by autoclaving unless noted otherwise. Buffers were titrated with sodium hydroxide or hydrochloride. Stock solutions were prepared freshly or stored at $-20^{\circ} \mathrm{C}$ if possible.

Buffers and media

5-fluoroorotic acid (5-FOA)

$0.67 \%(w / v)$ bacto yeast nitrogen base without amino acids, $0.2 \%(\mathrm{w} / \mathrm{v})$ SC-Ura drop out mix, 2 $\%(w / v)$ glucose, $0.01 \%(w / v)$ uracil, $0.1 \%(w / v)$ 5-FOA, medium was filter sterilized, for plates 5FOA medium was supplemented with autoclaved solution of bacto agar to $2 \%(\mathrm{w} / \mathrm{v})$ bacto agar final

DNA loading dye (6x) $10 \mathrm{mM}$ Tris/HCl pH8, $50 \mathrm{mM}$ EDTA, $1 \%$ (w/v) SDS, $30 \%(\mathrm{v} / \mathrm{v})$ glycerol, $0.1 \%(\mathrm{w} / \mathrm{v})$ bromophenol blue, $0.1 \%(\mathrm{w} / \mathrm{v})$ xylencyanol

Ficoll buffer $18 \%$ (w/v) Ficoll-400, $10 \mathrm{mM}$ Tris/HCl pH 7.5, 20 $\mathrm{mM} \mathrm{KCl,} 5 \mathrm{mM} \mathrm{MgCl}$, 3 mM DTT, 1 mM EDTA Lämmli running buffer (10x) $250 \mathrm{mM}$ Tris, 1,92 M glycine, 0,1\% (w/v) SDS LB medium $1 \%(\mathrm{w} / \mathrm{v})$ bacto tryptone, $0.5 \%(\mathrm{w} / \mathrm{v})$ yeast extract, $1 \%(\mathrm{w} / \mathrm{v}) \mathrm{NaCl}, \mathrm{pH} 7$, for agar plates LB medium was supplemented with $1.5 \%(\mathrm{w} / \mathrm{v})$ bacto agar PBS-Tween PBS supplemented with $0.2 \%(v / v)$ Tween 20

Phosphate buffered saline (PBS) $140 \mathrm{mM} \mathrm{NaCl}, 2.7 \mathrm{mM} \mathrm{KCl}, 10 \mathrm{mM} \mathrm{Na}{ }_{2} \mathrm{HPO}_{4}, 1.5$ $\mathrm{mM} \mathrm{KH}_{2} \mathrm{PO}_{4}, \mathrm{pH} 7.5$

pull-down buffer $50 \mathrm{mM}$ Tris $\mathrm{pH}$ 7.4, $200 \mathrm{mM} \mathrm{NaCl}, 1 \mathrm{mM} \mathrm{MgCl}, 5$ $\%$ (v/v) glycerol and $2 \mathrm{mg} / \mathrm{ml}$ BSA or ovalbumine 
SC complete

SC-X (X = Ura, Leu, His or Trp)

SDS sample buffer

SOC medium

Spheroblasting buffer

Sumoylation assay buffer (SAB)

TAE

Transport buffer (TB)

Western blot transfer buffer

YPD
$0.67 \%(w / v)$ bacto yeast nitrogen base without amino acids, $0.2 \%(\mathrm{w} / \mathrm{v})$ SC complete mix, $2 \%$ (w/v) glucose, $0.005 \%$ (w/v) adenine, SC complete medium for fluorescence analysis of cells was filter sterilized

$0.67 \%(w / v)$ bacto yeast nitrogen base without amino acids, $0.2 \%(\mathrm{w} / \mathrm{v}) \mathrm{SC}-\mathrm{X}$ drop out mix, $2 \%$ (w/v) glucose, $0.005 \%(w / v)$ adenine, SC-X medium was filter sterilized, for plates SC-X medium without adenine was supplemented with autoclaved solution of bacto agar to $2 \%(\mathrm{w} / \mathrm{v})$ bacto agar final

$50 \mathrm{mM}$ Tris/HCl pH 6.8, 2 \% (w/v) SDS, $0.1 \%$ (w/v) bromophenol blue, $10 \%$ (v/v) glycerol, 100 mM DTT, final, prepared as $1 \mathrm{x}, 2 \mathrm{x}$ and $4 \mathrm{x}$ stock solutions

$2 \%(\mathrm{w} / \mathrm{v})$ tryptone, $5 \%(\mathrm{w} / \mathrm{v})$ yeast extract, $50 \mathrm{mM}$ $\mathrm{NaCl}, 2.5 \mathrm{mM} \mathrm{KCl}, 10 \mathrm{mM} \mathrm{MgCl} 2,10 \mathrm{mM} \mathrm{MgSO}_{4}$ $50 \mathrm{mM}$ Tris/ $\mathrm{HCl} \mathrm{pH} \mathrm{7.5,} 10 \mathrm{mM} \mathrm{MgCl} 2,1 \mathrm{M}$ sorbitol, $1 \mathrm{mM}$ DTT

transport buffer supplemented with $0.2 \mathrm{mg} / \mathrm{ml}$ ovalbumine, $0.05 \%$ (v/v) Tween 20, 1 mM DTT, aprotinin, leupeptin, pepstatin

$40 \mathrm{mM}$ Tris acetate $\mathrm{pH}$ 7.7, $1 \mathrm{mM}$ EDTA

$20 \mathrm{mM}$ Hepes pH 7,3, 110 mM KOAc, 2 mM $\mathrm{Mg}(\mathrm{OAc})_{2}, 1 \mathrm{mM}$ EDTA, $2 \mathrm{mM}$ DTT, $1 \mu \mathrm{g} / \mathrm{ml}$ of each aprotinin, leupeptin, pepstatin 25 mM Tris/HCl, 193 mM glycine, 20\% (v/v) methanol, 0,036\% (v/v) SDS $1 \%(\mathrm{w} / \mathrm{v})$ bacto yeast extract, $2 \%(\mathrm{w} / \mathrm{v})$ bacto peptone, $2 \%$ (w/v) glucose, for plates YPD medium was supplemented with $2 \%(\mathrm{w} / \mathrm{v})$ bacto agar 
Stock Solutions

Ampicillin

Aprotinin (1000x)

ATP

Chloramphenicol

Coomassie destainer

Coomassiestaining solution

Digitonin

Dithiothreitol (DTT)

Gel drying solution

Hoechst 33258

Kanamycin

Leupeptin/Pepstatin (1000x)

PMSF

Ponceau-S

P1

P2

$\mathrm{P} 3$

Silver gel fixing solution

Silver gel developing solution

TAE (50x)

TFB-I

TFB-II
$100 \mathrm{mg} / \mathrm{ml}$

$1 \mathrm{mg} / \mathrm{ml}$

$100 \mathrm{mM}$ in $100 \mathrm{mM} \mathrm{Mg}(\mathrm{OAc})_{2}, 20 \mathrm{mM}$ HEPES, $\mathrm{pH} 7.4$

$30 \mathrm{mg} / \mathrm{ml}$

$50 \%(\mathrm{v} / \mathrm{v})$ methanol, $10 \%(\mathrm{v} / \mathrm{v})$ acetic acid $50 \%(\mathrm{v} / \mathrm{v})$ methanol, $10 \%(\mathrm{v} / \mathrm{v})$ acetic acid, $2.5 \%(\mathrm{w} / \mathrm{v})$ Brill. Blue R250

$10 \%(w / v)$ in DMSO

$1 \mathrm{M}$

$20 \%(\mathrm{v} / \mathrm{v})$ ethanol, $1 \%(\mathrm{v} / \mathrm{v})$ glycerol

$0.1 \mathrm{mg} / \mathrm{ml}$

$50 \mathrm{mg} / \mathrm{ml}$

$1 \mathrm{mg} / \mathrm{ml}$ each in DMSO

$100 \mathrm{mM}$ in 2-propanol

0,5 \% (w/v) Ponceau-S, $1 \%$ (v/v) acetic acid $50 \mathrm{mM}$ Tris/ $\mathrm{HCl} \mathrm{pH}$ 8.0, $10 \mathrm{mM}$ EDTA, $100 \mu \mathrm{g} / \mathrm{ml}$ RNase A $200 \mathrm{mM} \mathrm{NaOH}, 1 \%$ (v/v) SDS $3 \mathrm{M} \mathrm{KOAc} \mathrm{pH} 5.5$

$50 \%(\mathrm{v} / \mathrm{v})$ ethanol, $12 \%(\mathrm{v} / \mathrm{v})$ acetic acid, $0.018 \%(w / v)$ formaldehyde $3 \%(\mathrm{w} / \mathrm{v}) \mathrm{Na}_{2} \mathrm{CO}_{3}, 0.018 \%(\mathrm{v} / \mathrm{v})$, formaldehyde, $0.0005 \%(\mathrm{w} / \mathrm{v}) \mathrm{Na}_{2} \mathrm{~S}_{2} \mathrm{O}_{3}$ $2 \mathrm{M}$ Tris acetate $\mathrm{pH} 7.7,0,05 \mathrm{M}$ EDTA $100 \mathrm{mM} \mathrm{RbCl}, 15 \%$ (v/v) glycerol, $0.5 \mathrm{mM}$ $\mathrm{LiCl}, \mathrm{pH} 5.8$

$10 \mathrm{mM}$ MOPS pH 7, $10 \mathrm{mM} \mathrm{RbCl}, 75 \mathrm{mM} \mathrm{CaCl}_{2}$, $15 \%(v / v)$ glycerol 
5. Cell lines

Bacterial strains

$\mathrm{DH} 5 \alpha$

F- \$80lacZ $\Delta$ M15 $\Delta$ (lacZYA-argF) U169 deoR recA1 endA1 hsdR17( $\left.r_{K^{-}}, m_{K^{+}}\right)$supE44 thi-1 gyrA96 relA1 $\lambda$ -

BL21 (DE3) F- ompT hsdS $\mathrm{B}_{\mathrm{B}}\left(\mathrm{r}_{\mathrm{B}}-\mathrm{m}_{\mathrm{B}^{-}}\right)$gal dcm $\lambda(\mathrm{DE} 3)$

BL21 (DE3) pLysS

F- ompT hsdS ${ }_{B}\left(r_{B}-m_{B^{-}}\right)$gal dcm $\lambda(D E 3)$ pLysS $\left(\mathrm{Cm}_{R}\right)$

Yeast strains

ESM356-1 (from Elmar Schiebel*)

(Spore2.1) MATa ura3-52 leu2 $\Delta 1$ his3 $\Delta 200$ $\operatorname{trp} 1 \triangle 63$ (ADE2) Ura- Trp- His- Leu- G418 ${ }^{\mathrm{S}}$

ESM356-1/pRS316UBA2/uba2A::nat UBA2 shuffle strain based on ESM356-1

Mammalian cell lines

adherent HeLa cells

HeLa suspension cells (CSH HeLa strain)

human cervix carcinoma cell line

$\mathrm{NIH} 3 \mathrm{~T} 3$ human cervix carcinoma cell line mouse embryonic fibroblast cell line

* Prof. Dr. Elmar Schiebel, ZMBH in Heidelberg

\section{Oligonucleotides}

Oligonucleotides for cloning

\begin{tabular}{|c|c|c|c|c|}
\hline ampli & acceptor & & \# & sequence \\
\hline CFP & pET28a & for & 327 & $\begin{array}{l}\text { ATTTAAGCTAGCATGGTGAGCAAGGGCGAGG } \\
\text { AGC }\end{array}$ \\
\hline & & rev & 328 & CGGGATCCCTTGTACAGCTCGTCCATGCCG \\
\hline YFP & $\mathrm{pET} 28 \mathrm{~b}$ & for & 995 & TGCTAGCAACATGGTGAGCAAGGGC \\
\hline & & rev & 996 & CGGATCCCCCTTGTACAGCTCGT \\
\hline GFP & $\begin{array}{l}\text { p413-Uba2, } \\
\text { p413-S.c.Uba2 }\end{array}$ & for & 1541 & AA CTCGAG ATGGTGAGCAAGGGCGAGGAGC \\
\hline & & rev & 1542 & $\begin{array}{l}\text { TTGGTACCCTACTCGTCCATGCCGAGAGTGAT } \\
\text { C }\end{array}$ \\
\hline
\end{tabular}




\begin{tabular}{|c|c|c|c|c|}
\hline \multirow[t]{2}{*}{ Aos1 } & pET28a-CFP & for & 991 & AGAATTCATGGTGGAGAAGGAGGAGGC \\
\hline & & rev & 992 & GTGCCTTGGCCCCAAGTGAAAGCTTAAA \\
\hline \multirow[t]{2}{*}{ Uba2 } & $\begin{array}{l}\text { pET28b, } \\
\text { pET28-YFP }\end{array}$ & for & 1091 & AACCATGGGGATGGCACTGTCGCGGGGGCTG \\
\hline & & rev & 994 & $\begin{array}{l}\text { TGCTAGCTCCATCTAATGCTATGACATCATCAA } \\
\text { G }\end{array}$ \\
\hline \multirow[t]{2}{*}{$\begin{array}{l}\text { Uba2- } \\
\text { YFP }\end{array}$} & pcDNA3.1(-) & for & 1410 & AACTCGAGGAAATGGCACTGTCGCG \\
\hline & & rev & 1413 & TTGAATTCCTACAGCTCGTCCATGC \\
\hline \multirow[t]{2}{*}{ Uba2 } & p413 & for & 1527 & AAACTAGTATGGCACTGTCGCGGGGGCTG \\
\hline & & rev & 1528 & TTGTCGACTCAATCTAATGCTATGACATCATC \\
\hline \multirow[t]{2}{*}{ S.c.Uba2 } & p413 & for & 1532 & AAACTAGTATGCCAAGGGAAACAAGTTTGG \\
\hline & & rev & 1533 & TTGTCGACTCAGTCTAATTCAACAATATCAGAA \\
\hline \multirow[t]{2}{*}{ S.c.Uba2 } & p413-GFP & for & 1532 & AA ACTAGTATGCCAAGGGAAACAAGTTTGG \\
\hline & & rev & 1534 & TTGTCGACGTCTAATTCAACAATATCAGAA \\
\hline \multirow[t]{2}{*}{ S.c.Uba2 } & p413-NES & for & 1532 & AAACTAGTATGCCAAGGGAAACAAGTTTGG \\
\hline & & rev & 1535 & $\begin{array}{l}\text { TTGTCGACTCATAGTGCTAGTGCTGCTAGTGC } \\
\text { TAG GTCTAATTCAACAATATCAGAA }\end{array}$ \\
\hline \multirow[t]{2}{*}{ S.c.Uba2 } & p413-NES-GFP & for & 1532 & AA ACTAGTATGCCAAGGGAAACAAGTTTGG \\
\hline & & rev & 1536 & $\begin{array}{l}\text { TTGTCGACTAGTGCTAGTGCTGCTAGTGCTAG } \\
\text { GTCTAATTCAACAATATCAGAA }\end{array}$ \\
\hline \multirow[t]{2}{*}{ natNT2 } & UBA2-deletion & for & 1543 & $\begin{array}{l}\text { CTTCAACAGACACACAAGATCAGGAGCGCTAC } \\
\text { GCCAAACAAGAAAAGAAAATGCGTACGCTGCA } \\
\text { GGTCGAC }\end{array}$ \\
\hline & & rev & 1544 & $\begin{array}{l}\text { ACTGCGAACCACGATTAAATAAATATATAGATA } \\
\text { CCTTTTCTTATTTATTAATCGATGAATTCGAGC } \\
\text { TCG }\end{array}$ \\
\hline \multirow[t]{2}{*}{ UBA2 } & pRS316 & for & 1539 & AAACTAGTGAGCTTTTTCTTTCСССССТTCAAG \\
\hline & & rev & 1540 & TTGTCGACGAAATTCAAGATCACGCAAGAGGG \\
\hline
\end{tabular}

Oligonucleotides for mutagenesis

\begin{tabular}{|c|c|c|c|}
\hline mutation(s) & & \# & sequence \\
\hline \multirow[t]{2}{*}{ Aos1-P192A } & for & 1118 & CCAAGGAGTAGAAGATGGGGCCGACACCAAGAGAGC \\
\hline & rev & 1119 & GCTCTCTTGGTGTCGGCCCCATCTTCTACTCCTTGG \\
\hline \multirow[t]{2}{*}{$\begin{array}{l}\text { Aos1- } \\
\text { KR195,196A }\end{array}$} & for & 1067 & $\begin{array}{l}\text { GAAGATGGGCCCGACACCGCGGCAGCAAAACTTGATT } \\
\text { CTTCTGAG }\end{array}$ \\
\hline & rev & 1068 & $\begin{array}{l}\text { CTCAGAAGAATCAAGTTTTGCTGCCGCGGTGTCGGGC } \\
\text { CCATCTTC }\end{array}$ \\
\hline \multirow[t]{2}{*}{$\begin{array}{l}\text { Aos1-KRAK195- } \\
\text { 198A }_{4}\end{array}$} & for & 1069 & $\begin{array}{l}\text { GGGCCCGACACCGCGGCAGCAGCACTTGATTCTTCT } \\
\text { GAGACAACG }\end{array}$ \\
\hline & rev & 1070 & $\begin{array}{l}\text { CGTTGTCTCAGAAGAATCAAGTGCTGCTGCCGCGGTG } \\
\text { TCGGGCCC }\end{array}$ \\
\hline
\end{tabular}




\begin{tabular}{|c|c|c|c|}
\hline \multirow[t]{2}{*}{$\begin{array}{l}\text { Aos1- } \\
\text { LD199,200A }\end{array}$} & for & 1120 & $\begin{array}{l}\text { CGACACCAAGAGAGCAAAAGCTGCTTCTTCTGAGACA } \\
\text { ACG }\end{array}$ \\
\hline & rev & 1121 & $\begin{array}{l}\text { CGTTGTCTCAGAAGAAGCAGCTTTTGCTCTCTTGGTGT } \\
\text { CG }\end{array}$ \\
\hline \multirow[t]{2}{*}{$\begin{array}{l}\text { Aos1- } \\
\text { KK207,208A } 2\end{array}$} & for & 1071 & $\begin{array}{l}\text { CTTCTGAGACAACGATGGTCGCAGCGAAGGTGGTCTT } \\
\text { CTGCCCTGC }\end{array}$ \\
\hline & rev & 1072 & $\begin{array}{l}\text { GCAGGGCAGAAGACCACCTTCGCTGCGACCATCGTT } \\
\text { GTCTCAGAAG }\end{array}$ \\
\hline \multirow[t]{2}{*}{$\begin{array}{l}\text { Aos1-KKKV207- } \\
210 A_{4}\end{array}$} & for & 1073 & $\begin{array}{l}\text { GAGACAACGATGGTCGCAGCGGCGGCGGTCTTCTGC } \\
\text { CCTGTTAAAGAAGC }\end{array}$ \\
\hline & rev & 1074 & $\begin{array}{l}\text { GCTTCTTTAACAGGGCAGAAGACCGCCGCCGCTGCG } \\
\text { ACCATCGTTGTCTC }\end{array}$ \\
\hline \multirow[t]{2}{*}{$\begin{array}{l}\text { Uba2- } \\
\text { RK610,611A }\end{array}$} & for & 1059 & $\begin{array}{l}\text { CGTCAGTGAAGAAGAGAGAAGCGCCGCGAGGAAATT } \\
\text { AGATGAGAAAGAG }\end{array}$ \\
\hline & rev & 1060 & $\begin{array}{l}\text { CTCTTTCTCATCTAATTTCCTCGCGGCGCTTCTCTCTT } \\
\text { CTTCACTGACG }\end{array}$ \\
\hline \multirow[t]{2}{*}{$\begin{array}{l}\text { Uba2-RKRK610- } \\
613 A_{4}\end{array}$} & for & 1061 & $\begin{array}{l}\text { GAAGAGAGAAGCGCCGCGGCGGCATTAGATGAGAAA } \\
\text { GAGAATCTC }\end{array}$ \\
\hline & rev & 1062 & $\begin{array}{l}\text { GAGATTCTCTTTCTCATCTAATGCCGCCGCGGCGCTT } \\
\text { СTCTCTTC }\end{array}$ \\
\hline \multirow[t]{2}{*}{$\begin{array}{l}\text { Uba2- } \\
\text { KR623,624A } 2\end{array}$} & for & 1063 & $\begin{array}{l}\text { GATGAGAAAGAGAATCTCAGTGCAGCGGCGTCACGTA } \\
\text { TAGAACAGAAGGAAGAGC }\end{array}$ \\
\hline & rev & 1064 & $\begin{array}{l}\text { GCTCTTCCTTCTGTTCTATACGTGACGCCGCTGCACT } \\
\text { GAGATTCTCTTTCTCATC }\end{array}$ \\
\hline \multirow[t]{2}{*}{$\begin{array}{l}\text { Uba2-KRSR623- } \\
626 \mathrm{~A}_{4}\end{array}$} & for & 1065 & $\begin{array}{l}\text { GAGAAAGAGAATCTCAGTGCAGCGGCGGCAGCTATA } \\
\text { GAACAGAAGGAAGAGC }\end{array}$ \\
\hline & rev & 1066 & $\begin{array}{l}\text { GCTCTTCCTTCTGTTCTATAGCTGCCGCCGCTGCACT } \\
\text { GAGATTCTCTTTCTC }\end{array}$ \\
\hline \multirow[t]{2}{*}{$\begin{array}{l}\text { S.c.Uba2- } \\
\text { KRTK619- } \\
\text { 622AATA }\end{array}$} & for & 1551 & $\begin{array}{l}\text { GAGGCGCCCAGTAACGCAGCGACAGCGTTAGTTAATG } \\
\text { AACCG }\end{array}$ \\
\hline & rev & 1552 & $\begin{array}{l}\text { CGGTTCATTAACTAACGCTGTCGCTGCGTTACTGGGC } \\
\text { GCCTC }\end{array}$ \\
\hline
\end{tabular}

7. Vectors and plasmids

Vectors

$\begin{array}{lll}\text { name } & \text { designated use } & \text { source } \\ \text { pECFP-C1 } & \text { amplification of ECFP } & \text { Clontech } \\ \text { pEYFP-C1 } & \text { amplification of EYFP } & \text { Clontech } \\ \text { pET11d } & \text { bacterial expression } & \text { Novagen } \\ \text { pET28a } & \text { bacterial expression } & \text { Novagen } \\ \text { pET28a-ECFP } & \text { bacterial expression } & \text { this work } \\ \text { pET28b } & \text { bacterial expression } & \text { Novagen }\end{array}$




\begin{tabular}{|c|c|c|}
\hline pET28b-EYFP & bacterial expression & this work \\
\hline pcDNA3.1(-) & mammalian expression & Invitrogen \\
\hline pGBT9 & yeast expression & Manfred Kögl* \\
\hline p413 & yeast expression & Elmar Schiebel ${ }^{\#}$ \\
\hline pRS316 & yeast expression & Elmar Schiebel ${ }^{\#}$ \\
\hline
\end{tabular}

Plasmids

All sequences, except for GFP-derivates, are coding for human proteins unless noted otherwise. Tags are always located at the respective terminus shown in the plasmid construct.

\begin{tabular}{|c|c|}
\hline name & source \\
\hline pET28a-His-Aos1 & (Pichler et al. 2002)* $^{*}$ \\
\hline pET28a-His-ECFP-Aos1 & this work \\
\hline pET28a-His-ECFP-Aos1-KR195,196A2 & this work \\
\hline pET28b-Uba2-EYFP-His & this work \\
\hline pET28b-Uba2-KR623,624A2-EYFP-His & this work \\
\hline pET28b-Uba2-His & this work, (Werner et al. 2009) \\
\hline pET11d-Uba2 & (Pichler et al. 2002)* $^{*}$ \\
\hline pcDNA3.1(-)-ECFP-Aos1 & this work \\
\hline pcDNA3.1(-)-ECFP-Aos1-KR195,196A 2 & this work \\
\hline pcDNA3.1(-)-ECFP-Aos1-KRAK195-198A 4 & this work \\
\hline pcDNA3.1(-)-ECFP-Aos1-KK207,208A2 & this work \\
\hline pcDNA3.1(-)-ECFP-Aos1-KKKV207-210A 4 & this work \\
\hline pcDNA3.1(-)-ECFP-Aos1-P192A & this work \\
\hline pcDNA3.1(-)-ECFP-Aos1-LD199,200A 2 & this work \\
\hline pcDNA3.1(-)-Uba2-EYFP & this work \\
\hline pcDNA3.1(-)-Uba2-RK610,611A2-EYFP & this work \\
\hline pcDNA3.1(-)-Uba2-RKRK610-613A4-EYFP & this work \\
\hline pcDNA3.1(-)-Uba2-KR623,624A2-EYFP & this work \\
\hline pcDNA3.1(-)-Uba2-KRSR623-626A 4 -EYFP & this work \\
\hline pET11d-S.c.Uba2 & Erica Johnson $\#$ \\
\hline
\end{tabular}




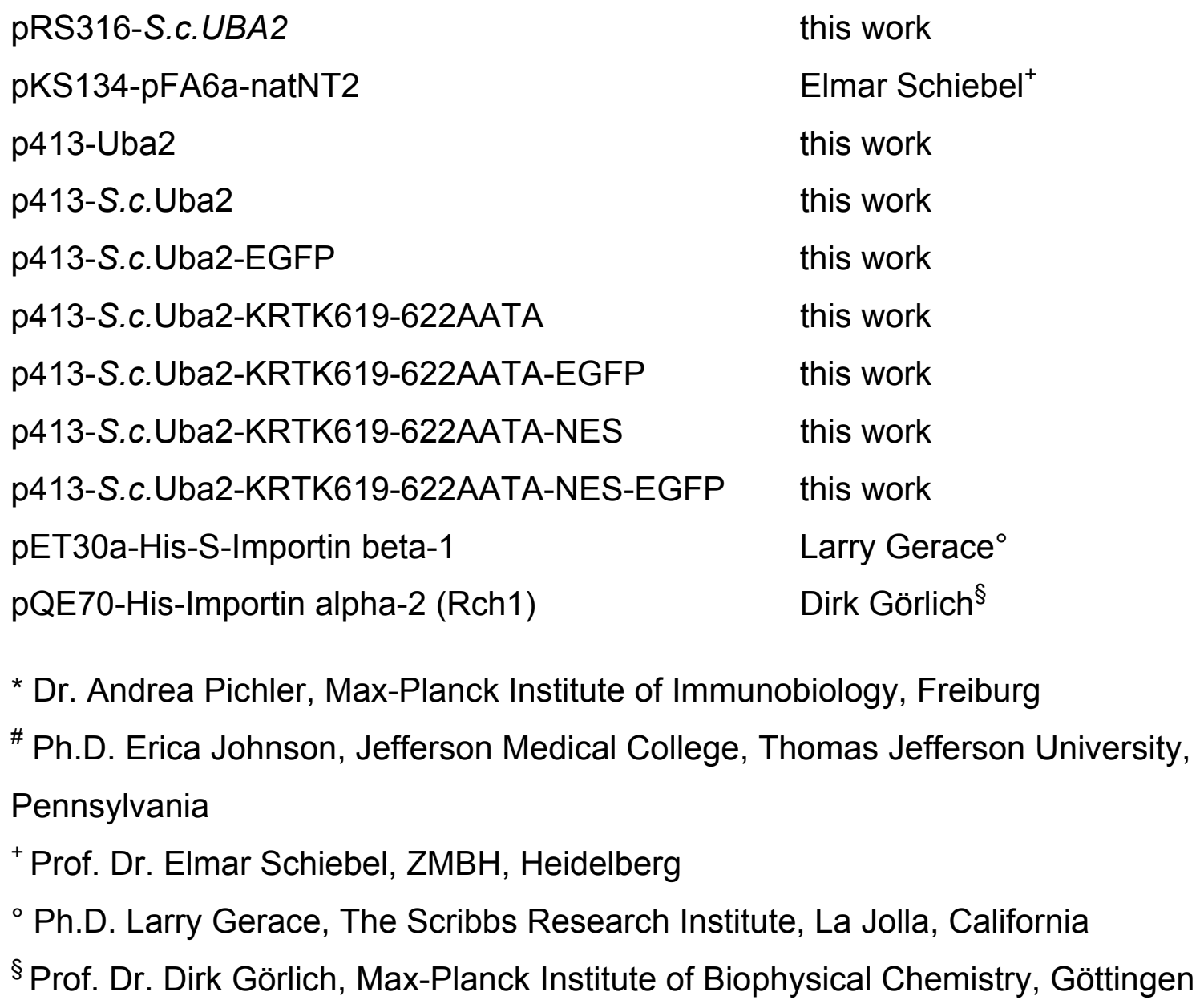

Generation of plasmids and vectors within this work pET28a-ECFP, pET28b-EYFP: ECFP was PCR amplified (\#327/328) from pECFPC1 and cloned into pET28a via Nhel and BamHI sites. Likewise EYFP was amplified (\#995/996) from pEYFP-C1 and cloned into Nhel and BamHI sites of pET28b.

pET28a-His-ECFP-Aos1 wt and -KR195,196A 2 : Human Aos1 was PCR amplified (\#991/992) from pET28a-Aos1 (Pichler et al., 2002) or pcDNA3.1(-)-KR195,196A2, respectively, and cloned into pET28a-ECFP via EcoRI and HindIII sites.

pET28b-Uba2-His, pET28b-Uba2-EYFP-His, pET28b-Uba2-KR623,624A2-EYFPHis: Human Uba2 wt and mutant were PCR amplified (\#1091/994) from pET11dUba2 (Pichler et al., 2002) or pcDNA3.1(-)-Uba2-KR623,624A $A_{2} E Y F P$, respectively, and cloned via Ncol and Nhel sites into pET28b and / or pET28b-EYFP.

pcDNA3.1(-)-ECFP-Aos1: ECFP-Aos1 was swapped from pET28a-His-ECFP-Aos1 into pcDNA3.1(-) via Nhel and HindllI sites.

pcDNA3.1(-)-ECFP-Aos1-P192A, -KR195,196A $\underline{2}_{2},-L D 199,200 A_{2}$ and $-K K 207,208 A_{2}:$ Indicated amino acids were changed to alanines by site directed mutagenesis on 
pcDNA3.1(-)-ECFP-Aos1 (P192 \#1118/1119, KR195,196 \#1067/1068, LD199,200 \#1120/1121, KK207,208 \#1071/1072).

pcDNA3.1(-)-ECFP-Aos1-KRAK195-198A 4 and -KKKV207-210A 4 : The indicated amino acids were changed to alanines in two steps, performing site-directed mutagenesis on already mutated templates. KRAK195-198 were changed by mutagenesis (\#1069/1070) on pcDNA3.1(-)-ECFP-Aos1-KR195,196A 2 and KKKV207-210 by further mutagenizing (\#1073/1074) pcDNA3.1(-)-ECFP-Aos1KK207,208A2.

pcDNA3.1(-)-Uba2-EYFP: Human Uba2-EYFP was PCR amplified (\#1410/1413) from pET28b-Uba2-EYFP-His and cloned into pcDNA3.1(-) via Xhol and EcoRI sites.

pcDNA3.1(-)-Uba2-RK610,611A 2 -EYFP and -KR623,624A 2 -EYFP: The indicated amino acids were changed to alanines by site directed mutagenesis (RK610,611 \#1059/1060, KR623,624 \#1063/1064) on pcDNA3.1(-)-Uba2-EYFP.

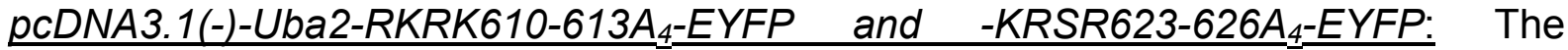
indicated amino acids were changed to alanines in two steps, performing sitedirected mutagenesis on already mutated templates. RKRK610-613 were changed by mutagenesis (\#1061/1062) on pcDNA3.1(-)-Uba2-RK610,611A2-EYFP and KRSR623-626 by mutagenizing (\#1065/1066) pcDNA3.1(-)-Uba2-KR623,624A EYFP.

pRS316-S.c.UBA2: The UBA2 gene from yeast, including approximately 500 bp in the 5'- and 3'-prime untranslated region, was PCR amplified (\#1539/1540) from isolated genomic yeast DNA provided by the lab of Elmar Schiebel $(\mathrm{ZMBH}$, University of Heidelberg) and cloned into pRS316 via Spel and Sall sites.

p413-Uba2, p413-S.c.Uba2: Human and yeast Uba2 were PCR amplified (human \#1527/1528, yeast \#1532/1533) from pET28b-Uba2 or pET11d-S.c.Uba2 and cloned via Spel and Sall sites into p413.

p413-S.C.Uba2-KRTK619-622AATA: The change of the indicated amino acids to alanines was performed by PCR amplification in two steps. First, two independent PCRs were performed to introduce the mutation, with reaction one (\#1532/1552) producing an amplicon from the 5'- start to the mutation site and reaction two (\#1551/1533) producing an amplicon from the mutation site to the 3'-end. Then, these amplicons were used as template DNA in a PCR (\#1532/1533) resulting in the complete final insert which was cloned into p413 via Spel and Sall sites. 
p413-S.c.Uba2-KRTK619-622AATA-NES: S.c.Uba2-KRTK619-622AATA was PCR amplified (\#1532/1535) from p413-S.c.Uba2-KRTK619-622AATA introducing a nuclear export sequence (NES) by the primer for the 3'-end. Then, the amplicon was cloned via Spel and Sall into $\mathrm{p} 413$.

p413-S.c.Uba2-EGFP: In the first step, a plasmid of p413 with yeast Uba2 not containing a stop-codon in the ORF was constructed. Therefore, yeast Uba2 was PCR amplified (\#1532/1534) from pET11d-S.c.Uba2 and cloned into p413 via Spel and Sall. Secondly, EGFP was introduced into the created construct via Sall and Xhol sites after PCR amplification (\#1541/1542) from pET28a-CFP.

p413-S.c.Uba2-KRTK619-622AATA-EGFP: This plasmid was created in a similar way to p413-S.c.Uba2-EGFP. First, a plasmid of yeast Uba2-KRTK619-622AATA without stop-codon was constructed by PCR amplification (\#1532/1534) and subsequent cloning of S.c.Uba2-KRTK619-622AATA via Spel and Sall in p413. Then, PCR amplified (\#1541/1542) EGFP was inserted into the created construct via Sall and Xhol sites.

p413-S.c.Uba2-KRTK619-622AATA-NES-EGFP: First, yeast Uba2-KRTK619622AATA-NES was PCR amplified (\#1532/1535) from p413-S.c.Uba2-KRTK619622AATA and cloned into p413 via Spel and Sall, generating a construct whose ORF did not contain a STOP-codon. Then, EGFP was PCR amplified (\#1541/1542) from $\mathrm{pET} 28 \mathrm{a}-\mathrm{CFP}$ and cloned via Sall and Xhol into the before generated plasmid.

\section{Recombinant proteins}

All recombinant proteins are, except for GFP-derivate tags human proteins unless noted otherwise. Tags are always located at the respective terminus indicated by the given name.

name

His-Aos1

His-ECFP-Aos1

His-ECFP-Aos1-KR195,196A 2

His-Aos1/Uba2

Uba2-His

Uba2-EYFP-His

Uba2-KR623,624A 2 -EYFP-His source

this work

this work

this work

this work

this work

this work

this work 
Ubc9 (mouse)

SUMO1 $\triangle \mathrm{C} 4$

RanGAP1 (mouse)

YFP-SUMO1 $1 \triangle \mathrm{C} 4$

CFP-GAPtail (mouse)

His-S-Importin beta 1

His-Importin alpha (Rch1)

His-Transportin

His-Importin 5

His-Importin 7 (xenopus)

His-Importin 9

His-Importin 13

His-Crm1

Ran, RanQ69L

His-YFP-M9 common stock ${ }^{*}$

common stock ${ }^{\star}$

common stock

common stock

common stock ${ }^{\star}$

this work

this work

Ralph Kehlenbach\#

Ralph Kehlenbach ${ }^{\#}$

Ralph Kehlenbach ${ }^{\#}$

Ralph Kehlenbach ${ }^{\#}$

Ralph Kehlenbach\#

Ralph Kehlenbach ${ }^{\#}$

Ralph Kehlenbach ${ }^{\#}$

Ralph Kehlenbach ${ }^{\#}$

*These proteins were purified alternatingly by members of the Melchior lab and are available as common protein stocks in the lab.

\# Dr. habil. Ralph Kehlenbach, University of Göttingen

\section{Antibodies}

Primary antibodies

\begin{tabular}{|c|c|c|c|}
\hline name & source & concentr. & working dilution \\
\hline goat $\alpha$-Aos 1 (affinity purified) & Melchior lab & $0.5 \mathrm{mg} / \mathrm{ml}$ & IF $1: 100$, WB $1: 1000$ \\
\hline goat $\alpha$-Uba2 (affinity purified) & Melchior lab & $0.5 \mathrm{mg} / \mathrm{ml}$ & IF $1: 100$, WB $1: 1000$ \\
\hline goat $\alpha-U b c 9$ (affinity purified) & Melchior lab & $1.5 \mathrm{mg} / \mathrm{ml}$ & WB 1: 500 \\
\hline goat $\alpha-G M 130$ clone 35 & BD Biosciences & $250 \mu \mathrm{g} / \mathrm{ml}$ & WB 1:250 \\
\hline goat $\alpha$-LDH & $\begin{array}{l}\text { Santa Cruz } \\
\text { Biochtechnology }\end{array}$ & $200 \mu \mathrm{g} / \mathrm{ml}$ & WB 1:500 \\
\hline goat $\alpha$-Nop1 & $\begin{array}{l}\text { Santa Cruz } \\
\text { Biotechnology }\end{array}$ & $200 \mu \mathrm{g} / \mathrm{ml}$ & WB 1:100 \\
\hline rabbit $\alpha-R 6 I P 2$ (ELKS) & Melchior lab & $0.5 \mathrm{mg} / \mathrm{ml}$ & WB 1:1000 \\
\hline
\end{tabular}




\begin{tabular}{|c|c|c|c|}
\hline rabbit $\alpha$-smt3 & Helle Ulrich* & unknown & WB 1:4000 \\
\hline mouse $\alpha-N M D A R 1$ & Nils Brose ${ }^{\#}$ & unknown & WB 1:1000 \\
\hline mouse $\alpha$-NTF97 clone 3E9 & Abcam & $\begin{array}{l}\text { unknown, } \\
\text { ascites }\end{array}$ & inhibiton studies $1: 2$ \\
\hline mouse $\alpha$-Pgk1 clone 22C5D8 & Molecular Probes & $1 \mathrm{mg} / \mathrm{ml}$ & WB 1:4000 \\
\hline mouse $\alpha$-RCC1 clone 9 & BD Biosciences & $250 \mu \mathrm{g} / \mathrm{ml}$ & WB $1: 1000$ \\
\hline
\end{tabular}

* Prof. Dr. Helle Ulrich, London Research Institue, London, UK

\# Prof. Dr. Nils Brose, Max-Planck Institute for Experimental Medicine, Göttingen

\section{Secondary antibodies}

Horseradish peroxidase-conjugated antibodies raised in donkey against IgGs from goat, mouse or rabbit were obtained from Dianova. They were used for western blot analysis in dilutions of 1:5,000-1:10,000.

Alexa488- and Alexa594-conjugated antibodies from donkey against goat IgG were obtained from Molecular Probes and used in an dilution of 1:500 - 1:1,000 for immunofluorescence analyses.

\section{Technical equipment and software}

Technical equipment

Bacterial incubator Kelvitron $\mathrm{t}$

Heraeus

Cell culture incubator Heracell Heraeus

Cell culture incubator Incucell MMM Medcenter

Centrifuges 5415C, 5424, 5415, 5430, 5417R Eppendorf

Chromatography system Äkta Purifier GE Healthcare

Confocal microscope CLSM510meta Zeiss

Electrophoresis Power Supply EPS300 Pharmacia Biotech

Elektrophoresis and blotting chambers EmulsiFlex-C5 Avestin

Film developing machine Curix 60 Agfa Fluoroskan Ascent microtiter plate reader Thermo Scientific Live cell imaging system CellObserver HighSpeed 
Microinjector

Eppendorf

NanoDrop ND1000

Thermo Scientific

Rotors TLA-100.3, JS-5.2, Type45Ti, Type60Ti, JA-20, Type70.1Ti Beckman Coulter

Scanner 4990 Photo

Epson

Sterile cell culture hood Herasafe

Heraeus

Thermocycler Primus

MWG Biotech

Thermocycler T3000 and Tprofessional

Biometra

Thermomixer Compact

Eppendorf

Ultracentrifuge Optima Max, Optima L-80 XP

Beckman Coulter

Vacuum pump LABOPORT N480.3FTP

KNF Neuberger

Vortex Genie 2

Scientific Industries

Software

Adobe acrobat 9 pro

Adobe

Adobe creative suite 4

Adobe

Axiovision software 4 Zeiss

BioMath Calculators

ClustalW2

http://www.promega.com/biomath/calc11.htm, Promega

Endnote $\mathrm{X} 2$ http://www.ebi.ac.uk/Tools/clustalw2/index.html, EMBL

PyMOL v0.99

Thomson Reuters

Rotor Calculator

DeLano Scientific LLC

SigmaPlot 8.02

Beckman Coulter

Vector NTI Systat Software Inc. Invitrogen 


\section{Methods}

Standard procedures in molecular biology, biochemistry and cell biology were performed according to basic methods described in Molecular Cloning: A Laboratory Manual (Maniatis et al. 1989), Current Protocols in Protein Science (Coligan et al. 2003), Current Protocols in Cell Biology (Bonifacino et al. 2000), and Cells: A Laboratory Manual (Spector et al. 1998).

\section{Molecular biology methods}

\subsection{Preparation of chemical competent bacteria}

Chemical competent E.coli were prepared from a $200 \mathrm{ml}$ growing culture with an optical density $\mathrm{OD}_{600 \mathrm{~nm}}$ of 0.5 . After 10 minutes incubation on ice, bacteria were harvested by centrifugation with $5,000 \mathrm{~g}$ at $4^{\circ} \mathrm{C}$. The cell pellet was resuspended in $200 \mathrm{ml}$ ice-cold TFB-I buffer and incubated on ice for 2 hours. The cells were again collected with $5,000 \mathrm{~g}$ at $4^{\circ} \mathrm{C}$ and resuspended in $8 \mathrm{ml}$ of sterile cold TFB-Il buffer. Aliquots of $100 \mu \mathrm{l}$ were frozen in liquid nitrogen and stored at $-80^{\circ} \mathrm{C}$.

\subsection{Transformation of competent bacteria}

Chemical competent E.coli were thawed on ice and incubated with DNA for 30 minutes on ice. Incorporation of the DNA was achieved by heat-shock at $42^{\circ} \mathrm{C}$ for 90 seconds, followed by incubation on ice for 2 minutes. For regeneration the bacteria were supplemented with $500 \mu \mathrm{LB}$ medium and incubated in a shaker at $37^{\circ} \mathrm{C}$ for 1 hour. Finally the transformed cells were plated on LB agar plates supplemented with the respective antibiotic for the transformed plasmid.

\subsection{Plasmid DNA purification}

Small amounts of plasmid DNA were prepared by alkaline lysis (Birnboim et al. 1979) and subsequent precipitation. $2 \mathrm{ml}$ overnight cultures of E.coli DH5 $\alpha$ were harvested for 5 minutes with $3,000 \mathrm{~g}$ at room temperature and resuspended in $300 \mu \mathrm{l}$ resuspension buffer P1. After mixing with $300 \mu \mathrm{l}$ alkaline lysis buffer P2 the lysate was incubated for 5 minutes at room temperature before proteins and chromosomal DNA were precipitated by the addition of $300 \mu$ neutralization buffer P3. The lysates were cleared from unsoluble components by centrifugation for 30 minutes with 
$13,000 \mathrm{~g}$ at $4^{\circ} \mathrm{C}$ and the plasmid DNA was precipitated from $800 \mu \mathrm{l}$ supernatant with $640 \mu \mathrm{l}$ 2-propanol. After collecting DNA by centrifugation for 15 minutes with 13,000 $\mathrm{g}$ at room temperature the DNA was washed with $70 \%(\mathrm{v} / \mathrm{v})$ ethanol, dried and resuspended in $30-50 \mu$ l water.

High quality isolation of DNA was necessary for plasmids used for transfection of cultured cells. Purification of small or large quantities of DNA was performed using NucleoBond PC 100 or PC500 Kit from Macherey \& Nagel according to the manufactorer's instructions.

\subsection{Measurement of DNA concentration}

The concentration of DNA was quantified by measuring the $O D_{260 \mathrm{~nm}}$, with an appropriate dilution ensuring that the $O D$ is in the dynamic range of the spectrometer. The ratio of $\mathrm{OD}_{260 \mathrm{~nm}}$ to $\mathrm{OD}_{280 \mathrm{~nm}}$, ideally 1.8 , revealed information about the purity of the DNA.

\subsection{Agarose gel electrophoresis}

DNA fragments of different size were separated by agarose gel electrophoresis. Depending on the fragment sizes $0.5-3 \%(\mathrm{w} / \mathrm{v})$ agarose were dissolved in $50 \mathrm{ml}$ TAE buffer by heating and the solid gel was formed by cooling down in a gel chamber. The gel was loaded with $8 \mu \mathrm{l}$ of $1 \mathrm{~kb}$ DNA marker and with DNA samples supplemented with an appropriate amount of 6x DNA loading dye. After seperation at 80 Volt DNA was stained in a bath with $1 \mu \mathrm{g} / \mathrm{ml}$ ethidiumbromide and was visualized with UV light of $365 \mathrm{~nm}$.

\subsection{Isolation of DNA from agarose gels}

After separation of DNA fragments by agarose gel electrophoresis bands were cut out of the gel and DNA fragments were extracted using NucleoSpin (Extract II Kit from Macherey \& Nagel according to the manufacturer's instructions. DNA was eluted in $20-40 \mu$ l elution buffer.

\subsection{Restriction of DNA by endonucleases}

The buffers and enzymes of Fermentas were used for DNA restriction. Reaction conditions were chosen according to the manufactorer's instructions for the 
respective enzymes used. In preparative restrictions $1 \mu \mathrm{g}$ DNA was converted in 100 $\mu$ reactions containing approximately $2-10$ units of enzyme for $2-4$ hours at $37^{\circ} \mathrm{C}$, analytical reactions were performed in $20 \mu$ reactions containing $200-500$ ng DNA. To prevent star-activity the amount of enzymes never exceeded $1 / 10$ of the total reaction volume.

\subsection{Ligation of DNA fragments}

Ligations were set up with vector to insert at a molar ratio of $1: 3-1: 5$ in $10 \mu \mathrm{l}$ reactions containing 1 unit T4 DNA ligase (Fermentas), ligation buffer and some additional ATP. Ligation was performed for $2 \mathrm{~h}$ at $30{ }^{\circ} \mathrm{C}$ or at $16{ }^{\circ} \mathrm{C}$ overnight and the reaction was transformed into $\mathrm{DH} 5 \alpha$.

\subsection{Sequencing of DNA}

All plasmids constructed via PCR amplification were verified by DNA sequencing based on the chain-teminating method in principle established by Sanger, Nicklen and Coulsen (Sanger et al. 1977). The sequencing reactions were performed with BigDye Terminator v1.1 cycle sequencing kit (Applied Biosystems) and contained 50 - 200 ng plasmid DNA, 10 pmol primer, $2 \mu$ sequencing buffer and $1 \mu$ l sequencing mix in a final volume of $10 \mu \mathrm{l}$. PCR was performed according to the following program: initial denaturation 2 minutes at $96^{\circ} \mathrm{C}$, denaturation during cycling 10 second at $96{ }^{\circ} \mathrm{C}$, annealing for 15 seconds at $55{ }^{\circ} \mathrm{C}$, elongation at $60{ }^{\circ} \mathrm{C}$ for 4 minutes, 25 cycles.

After stopping the reaction with $1 \mu \mathrm{l}$ of each $3 \mathrm{M} \mathrm{Na}(\mathrm{OAc}) \mathrm{pH} 5.2$ and $125 \mathrm{mM}$ EDTA the PCR product was precipitated by addition of $50 \mu \mathrm{l}$ ice cold absolute ethanol and centrifugation for 15 minutes with $13,000 \mathrm{~g}$. The pellet was washed with $70 \%(\mathrm{v} / \mathrm{v})$ ethanol, was dried and was resuspended in $30 \mu \mathrm{l}$ sterile water. Read-out was performed in a Genetic Analyzer 3100 (Applied Biosystems) at the Göttinger Zentrum für Molekulare Biowissenschaften (GZMB) of the University of Göttingen.

Alternatively sequencing was carried out by GATC Biotech from $30 \mu \mathrm{l}$ of $30-100$ $\mathrm{ng} / \mu \mathrm{l}$ plasmid DNA and $10 \mathrm{pmol} / \mu \mathrm{l}$ primer.

The sequences were analyzed using Lasergene Gene Quest (DNAstar) or a free of charge version of the software Vector NTI (Invitrogen). 


\subsection{Polymerase chain reaction (PCR)}

The principle of the polymerase chain reactions traced back to K.B. Mullis (Mullis 1990). Amplification of specific DNA fragments was performed by PCR with two primers defining beginning and end of the amplicon. Reactions were set up in a final volume of $50 \mu \mathrm{l}$ with $50-100 \mathrm{ng}$ template DNA or $1-2 \mu \mathrm{l}$ cDNA, 20 pmol of each forward and reverse primer, $200 \mu \mathrm{l}$ of each dNTP and 1 unit Phusion (Finnzymes) or Vent (NEB) polymerase. In the case of GC-rich DNA $3 \%(\mathrm{v} / \mathrm{v})$ DMSO were added to the reaction. The annealing temperature for the appropriate oligonucleotide was calculated according to a web-based calculator (http://www.promega.com/biomath/ calc11.htm). $3{ }^{\circ} \mathrm{C}$ were added to the calculated temperature when phusion polymerase was used, whereas $5-6{ }^{\circ} \mathrm{C}$ were substracted when DMSO was added to the reaction. Amplification time was chosen in dependence of the processivity of the used polymerase. In general, amplification was performed according the following program:

initial denaturation 1 minute $95{ }^{\circ} \mathrm{C}$, denaturation during cycling 30 seconds $95{ }^{\circ} \mathrm{C}$, annealing for 30 seconds at the calculated temperature, elongation at $72{ }^{\circ} \mathrm{C}$ for the calculated time, standardwise 35 cycles and an additional final elongation step at 72 ${ }^{\circ} \mathrm{C}$ for $2-5$ minutes.

\subsection{Site directed mutagenesis}

PCR reactions for site directed in vitro-mutagenesis of plasmids were based on the protocol QuickChange Site-Directed Mutagenesis Kits from Stratagene. The used oligonucleotides were reverse-complementary to each other and comprised the desired mutations. Reactions of $50 \mu$ final volume contained $25 \mathrm{ng}$ plasmid DNA, 5 pmol of each primer, $5 \mathrm{nmol}$ of each dNTP, $2.5 \mu \mathrm{l}$ 10fold Pfu buffer and 1.5 units Pfu DNA polymerase. Amplification was performed according to the following program: initial denaturation 2 minutes $95^{\circ} \mathrm{C}$, denaturation during cycling 30 seconds $95{ }^{\circ} \mathrm{C}$, annealing for 1 minute at the calculated temperature (see above), elongation at $72{ }^{\circ} \mathrm{C}$ for the calculated time, standardwise 28 cycles and an additional final elongation step at $72{ }^{\circ} \mathrm{C}$ for $2-5$ minutes. Subsequently the reaction was cooled to $4{ }^{\circ} \mathrm{C}$, incubated with 10 units $\mathrm{Dpnl}$ for $1 \mathrm{~h}$ at $37^{\circ} \mathrm{C}$ for selective degradation of the methylated template DNA and transformed into competent bacteria. 


\section{Biochemical methods}

\subsection{Measurement of protein concentration}

The concentration of protein solutions was determined with a BCA-test essentially established by Smith et al. (Smith et al. 1985). In alkaline solution proteins form a complex with $\mathrm{Cu}^{2+}$ ions (Biuret-reaction) and the reduced ions subsequently form a violet complex with bicinchinoninic acid (BCA) that can be measured at $562 \mathrm{~nm}$. Concetrations were measured using BCA Protein Assay kit (Pierce) according to the manufacturer's instructions. As standard the result was confirmed by comparison to different defined amounts of BSA analyzed in the same Coomassie stained gel.

\subsection{SDS PAGE and detection of proteins}

SDS polyacrylamide gel electrophoresis (SDS PAGE)

Separation of proteins was performed by SDS polyacrylamide gel electrophoresis essentially according to the system described by Laemmli (Laemmli 1970). In most cases $10 \%$ gels or continous $5-20 \%$ gradient gels were used. Gels were prepared in blocks of eight gels, first casting the separation gel containing $10 \%(w / v)$ polyacrylamide (for $10 \%$ gels) in $0.4 \mathrm{M}$ Tris- $\mathrm{HCl} \mathrm{pH} 8.8,0.1 \%$ (w/v) SDS, $0.001 \%$ $(\mathrm{w} / \mathrm{v})(\mathrm{v} / \mathrm{v})$ of each APS and TEMED; an overlay of 2-propanol assured an even surface. Gradient gels were prepared using a double-cylindrical gradient mixer, each cylinder filled with equal amounts of $5 \%$ and $20 \%(\mathrm{w} / \mathrm{v})$ polyacrylamide solutions. The mixture of these solutions was filled into the block resulting in a continous gradient of $5 \%$ at the top and $20 \%$ towards the bottom of the gels. After polymerization of the separation gel the 2-propanol was removed and the stacking gel consisting of $4 \%(\mathrm{w} / \mathrm{v})$ polyacrylamide, $50 \mathrm{mM}$ Tris- $\mathrm{HCl} \mathrm{pH} 6.8,0.1 \%(\mathrm{w} / \mathrm{v}) \mathrm{SDS}$, $0.001 \%(w / v)$ APS and $0.001 \%(v / v)$ TEMED was poured on top of the separation gel. After insertion of combs the stacking gel polymerized for approximately 2 hours. The gels were run with Laemmli buffer at $20 \mathrm{mAmp} / 300 \mathrm{~V}$ per gel at room temperature.

Before loading the samples were adjusted to approximately $50 \mathrm{mM}$ Tris- $\mathrm{HCl} \mathrm{pH} 6.8$, $2 \%(\mathrm{w} / \mathrm{v})$ SDS, $0.1 \%$ (w/v) bromophenol blue, $10 \%$ glycerol, $100 \mathrm{mM}$ DTT with one, two-, or four-fold concentrated SDS sample buffer and were boiled at $95{ }^{\circ} \mathrm{C}$ for a few minutes. DTT sensitive samples like thioester bonds were mixed 1:1 with TLB 
(50 mM Tris pH 6.8, 2 \% SDS, 4 M Urea, $10 \%$ glycerol) supplemented with $0.1 \%$ $(\mathrm{w} / \mathrm{v})$ bromophenol blue and were incubated for 10 minutes at $60^{\circ} \mathrm{C}$.

\section{Coomassie staining}

For Coomassie staining, gels were stained for at least 1 hour in Coomassie-staining solution and then incubated in Coomassie destaining solution until all excess dye was removed from the gel.

Alternatively gels were fixed in a solution of $40 \%$ ethanol and $10 \%$ acetic acid for 20 - 30 minutes, the gels were rehydrated for some minutes in water and were stained in a solution of $10 \%$ acetic acid and $0.005 \%$ Coomassie R-250. In this case, destaining of the gel due to high background was not necessary.

For documentation gels were incubated for $30-60$ minutes in gel drying solution, mounted between cellophane sheets and clamped between two plastic frames for drying.

\section{Silver staining}

Detection of small amounts of proteins in gels was achieved by silver staining, a method principally developed by Merril et al. (Merril et al. 1981). For this, the gel was fixed for at least 1.5 hours in fixing solution, washed three times for 20 minutes in 50 $\%(\mathrm{v} / \mathrm{v})$ ethanol, incubated in fresh $0.01 \%(\mathrm{w} / \mathrm{v})$ disodium-thiosulfate $\mathrm{Na}_{2} \mathrm{~S}_{2} \mathrm{O}_{3}$ for 1 minute, washed three times for 20 seconds in water and stained for 20 minutes in fresh $0.1 \%(\mathrm{w} / \mathrm{v})$ silver nitrate $\mathrm{AgNO}_{3}$. The stained gels were developed by incubation in developing solution for up to 10 minutes. When the protein bands became visible, the gel was washed with water and the reaction was stopped with 10 mM EDTA.

\section{Immunoblotting}

Prior to immunological detection, the proteins were transferred in a semi-dry western blot apparatus from the gel onto a nitrocellulose membrane with a pore size of 0.2 $\mu \mathrm{m}$ (Kyhse-Andersen 1984). Three layers of Whatman 3MM paper, a nitrocellulose membrane, the gel and again three layers Whatman paper soaked in transfer buffer were prepared from anode to kathode. Protein transfer was executed with 20 volt for 1 - 2 hours depending on the size of the protein of interest. The transfer was controlled by staining protein with Ponceau-S solution and excess dye was removed by washing with $1 \%(\mathrm{v} / \mathrm{v})$ acetic acid. 
Unspecific binding sites on the membrane were blocked by incubation with blocking buffer (5 \% (w/v) skim milk in PBS-Tween) for 1 hour at room temperature. Appropriate dilutions of primary and HRP-coupled secondary antibody in blocking buffer were prepared; the blot was incubated with primary antibody for at least 1 hour at room temperature or overnight at $4{ }^{\circ} \mathrm{C}$, washed three times for 15 minutes with PBS-Tween and then incubated with secondary antibody for $1-2$ hours at room temperature. After removing unbound antibody by washing with PBS-Tween, bound antibody was detected by chemiluminescence using ECL kits from Pierce or Millipore. Exposure times of the films were chosen according to the strength of the signal; films were developed using an automatic developing machine.

\subsection{Protein precipitation with TCA}

To concentrate protein solutions for SDS PAGE, proteins were precipitated and redissolved. Precipitation was performed by mixing the protein solution with an equal volume of $20 \%(\mathrm{w} / \mathrm{v})$ trichloroacetic acid (TCA), followed by incubation for 30 minutes on ice. The precipitated proteins were collected by centrifugation with 14,000 $x \mathrm{~g}$ for 30 minutes at $4{ }^{\circ} \mathrm{C}$, the TCA solution was completely removed and the protein was dissolved in 4 x SDS sample buffer facilitated by sonication.

\subsection{Expression and purification of recombinant proteins}

Recombinant Aos1, Uba2 or E1 complexes were expressed and purified according to the detailed protocol of Werner et al. (Werner et al. 2009); the following paragraphs briefly describe the applied procedures.

\section{Expression and purification of recombinant Aos1}

For recombinant expression of N-terminally His- or His-CFP-tagged Aos1 the respective pET28a expression construct was transformed into $E$. coli strain BL21(DE3) and directly used for inoculation of LB medium containing $30 \mu \mathrm{g} / \mathrm{ml}$ kanamycin. After growth over-night at $37^{\circ} \mathrm{C}$, bacteria were harvested by centrifugation, resuspended in fresh medium and grown at $37{ }^{\circ} \mathrm{C}$ until they reached an $\mathrm{OD}_{600 \mathrm{~nm}}$ of 0,6 . Then, protein expression was induced by addition of a final concentration of $1 \mathrm{mM}$ IPTG and bacteria were grown for another 6 hours at $25^{\circ} \mathrm{C}$. 
After harvesting by centrifugation at 4000 rpm in a Beckmann JS-5.2 rotor, bacteria were resuspended in $50 \mathrm{ml} \mathrm{E1}$ lysis buffer $(50 \mathrm{mM}$ Na-phosphate $\mathrm{pH}$ 8.0, $300 \mathrm{mM}$ $\mathrm{NaCl}, 10 \mathrm{mM}$ imidazole), flash frozen in liquid nitrogen and stored at $-80^{\circ} \mathrm{C}$.

Once thawed, $1 \mathrm{mM} \beta$-mercaptoethanol, $0.1 \mathrm{mM} \mathrm{PMSF}$ and $1 \mu \mathrm{g} / \mathrm{mL}$ each of aprotinin, leupeptin and pepstatin were added before cells were lyzed using an emulsion flex. Bacterial debris was removed by centrifugation at $100,000 \times \mathrm{g}$ at $4{ }^{\circ} \mathrm{C}$ for $1 \mathrm{~h}$ in a Beckman Type 45 Ti rotor. Per 1 I bacterial culture $1 \mathrm{ml}$ pure ProBond Nickel-chelating resin (Invitrogen) was transferred into a column and equilibrated in E1 lysis buffer supplemented with $1 \mathrm{mM} \beta$-mercaptoethanol and $1 \mu \mathrm{g} / \mathrm{mL}$ AP and LP. The protein supernatant was applied to the column at $4{ }^{\circ} \mathrm{C}$ and His-Aos 1 was collected on the beads by gravity flow. The column was extensively washed with E1 wash buffer (50 mM Na-phosphate, pH 8.0, 300 mM NaCl, 20 mM imidazole, $1 \mathrm{mM}$ $\beta$-mercaptoethanol, $1 \mu \mathrm{g} / \mathrm{ml}$ AP and LP) until no more proteins were detected in the flow-through. Bead-bound proteins were eluted with at least 5 column volumes elution buffer (50 mM Na-phosphate, pH 8.0, $300 \mathrm{mM} \mathrm{NaCl}, 250 \mathrm{mM}$ imidazole, 1 $\mathrm{mM} \beta$-mercaptoethanol, $1 \mu \mathrm{g} / \mathrm{ml} \mathrm{AP}$ and LP) and collected in $1.5 \mathrm{ml}$ fractions. Protein-containing fractions were combined and concentrated to approximately $5 \mathrm{ml}$. After filtration of the concentrate through a $0.2 \mu \mathrm{m}$ low protein binding filter, the solution was injected onto a HiLoad 26/60 Superdex 200 pg column (GE Healthcare) equilibrated in S200 buffer $(50 \mathrm{mM}$ Tris- $\mathrm{HCl}, \mathrm{pH} 7.5,50 \mathrm{mM} \mathrm{NaCl}, 1 \mathrm{mM}$ DTT, 1 $\mu \mathrm{g} / \mathrm{ml} \mathrm{AP}$ and LP). $5 \mathrm{ml}$ fractions were collected, analyzed by SDS-PAGE and fractions containing His-Aos1 were combined and dialyzed against TB. If necessary, the purified protein was concentrated and aliquots were flash frozen in liquid nitrogen and stored at $-80{ }^{\circ} \mathrm{C}$. Using to this protocol approximately $5-7 \mathrm{mg}$ His-Aos 1 or HisCFP-Aos can be purified per liter of $E$. coli culture.

\section{Expression and purification of recombinant Uba2}

For expression of C-terminally His- or YFP-His-tagged Uba2 the respecitve pET28b expression construct was tranformed into chemically competent BL21(DE3) and streaked on LB agar plates containing $30 \mu \mathrm{g} / \mathrm{ml}$ kanamycin. LB medium supplemented with kanamycin was inoculated with a single colony and grown overnight for approximately 18 hours at $37^{\circ} \mathrm{C}$. Protein induction and bacterial lysis as well as the purification step via ProBond resin and preparative Superdex 200 gel filtration column (GE Healthcare) exactly corresponded to the description of the purification of 
Aos1. However, purification of Uba2 requires one additional purification step. Therefore, Uba2 containing fractions after gel filtration chromatography were combined and applied to a MonoQ anion-exchange column (GE Healthcare) equilibrated with $S 200$ buffer. Elution from the MonoQ was performed by a linear gradient of $50 \mathrm{mM}$ to $500 \mathrm{mM} \mathrm{NaCl}$ in S200 buffer. Fractions of $0.5 \mathrm{ml}$ were collected and analyzed for Uba2 content by SDS-PAGE and Coomassie staining. Despite a predicted size of $72 \mathrm{kDa}$, untagged Uba2 migrates at $90 \mathrm{kDa}$ in SDS PAGE and tagged protein correspondingly slower. Combined fractions were concentrated, dialyzed against TB and aliquots were flash-frozen in liquid nitrogen and stored at -80 ${ }^{\circ} \mathrm{C}$. Purification results in a yield of approximately $1.5-3 \mathrm{mg}$ Uba2-His or Uba2-YFPHis per liter E. coli culture.

\section{Expression and purification of recombinant SUMO E1 enzyme}

SUMO-E1 complex can be obtained by two different approaches. The classical protocol is based on co-expression of N-terminally His-tagged Aos 1 and untagged Uba2, whereas an alternative approach developed as part of this thesis $\mathrm{N}$-terminally His-tagged Aos1 and C-terminally His-tagged Uba2 are independently purified as single subunits and E1 complex is subsequently reconstituted.

For the classical E1 purification pET28a-His-Aos1 and pET11d-Uba2 expression constructs are co-transformed into BL21(DE3) and immediately used for inocculation of LB medium supplemented with $50 \mu \mathrm{g} / \mathrm{ml}$ ampicillin and $30 \mu \mathrm{g} / \mathrm{ml}$ kanamycin. The subsequent procedure exactly matches the previously described protocol for expression and purification of Uba2. The resulting fractions of the MonoQ purification step are analyzed by SDS-PAGE and Coomassie staining and fractions containing equal amounts of His-Aos1 and Uba2 were combined and processed as described.

In the alternative protocol equimolar amounts of purified His-Aos1 and Uba2-His were incubated in TB on ice for $1-2 \mathrm{~h}$ in order to form heterodimeric $\mathrm{E} 1 \mathrm{complex}$. To separate assembled complex from any excess of one subunit or of partially unfolded protein His-Aos1/Uba2-His was subsequently purified by a preparative Superdex 200 gel filtration, flash frozen in liquid nitrogen and stored at $-80^{\circ} \mathrm{C}$.

\section{Expression and purification of recombinant import receptors}

His-Importin $\alpha$ was expressed and purified essentially as described by $\mathrm{Hu}$ et al. (Hu et al. 1996). Briefly, BL21 cells were transformed with the expression construct and streaked on LB agar containing ampicillin. Subsequently LB supplemented with 50 
$\mu \mathrm{g} / \mathrm{ml}$ ampicillin was inocculated with all colonies of the plate of BL21 cells. The bacterial culture was grown at $37^{\circ} \mathrm{C}$ until an $\mathrm{OD}_{600 \mathrm{~nm}}$ of 0.6 was reached and recombinant protein was expressed in the presence of $1 \mathrm{mM}$ IPTG at $25^{\circ} \mathrm{C}$ for $5-6$ hours. Cells were harvested, resuspended in lysis buffer (50 mM Tris, pH 8.0, 500 $\mathrm{mM} \mathrm{NaCl}$ ) and stored at $-80{ }^{\circ} \mathrm{C}$. After addition of $5 \mathrm{mM} \circledast$-mercaptoethanol, $1 \mathrm{mM}$ PMSF and $1 \mu \mathrm{g} / \mathrm{ml}$ AP, LP bacteria were lyzed using an Emulsion flex and subjected to ultracentrifugation. After addition of $10 \mathrm{mM}$ imidazol the supernatant was transferred onto an equilibrated column of $0.5 \mathrm{ml} \mathrm{Ni-beads} \mathrm{per} \mathrm{liter} \mathrm{of} \mathrm{bacterial}$ culture, washed with $12 \mathrm{mM}$ imidazol in lysis buffer and eluted with S200 buffer (20 $\mathrm{mM}$ Tris, $\mathrm{pH}$ 7.5, $150 \mathrm{mM} \mathrm{NaCl}, 1 \mathrm{mM}$ DTT) containing $250 \mathrm{mM}$ imidazol. Proteincontaining fractions were combined, concentrated and injected onto a Superdex 200 preparative column equilibrated and run with $\$ 200$ buffer. Fractions containing importin $\alpha$ were combined, concentrated, flash-frozen in liquid nitrogen and stored at $-80{ }^{\circ} \mathrm{C}$.

Purification of His-S-Importin $\beta$ was performed according to Chi et al. (Chi et al. 1997). The expression construct was transformed into BL21(DE3)-pLysS, streaked on a LB agar plate containing chloramphenicol and kanamycin and LB medium supplemented with kanamycin was inocculated with all colonies from the fresh plate. The culture was grown at $37^{\circ} \mathrm{C}$ to $\mathrm{OD}_{600 \mathrm{~nm}}$ of $0.3-0.4$, temperature was adjusted to $20{ }^{\circ} \mathrm{C}$ and at $\mathrm{OD}_{600 \mathrm{~nm}}$ of 0.6 protein expression was induced by addition of $1 \mathrm{mM}$ IPTG. After expression for 4 hours, bacteria were harvested, resuspended in lysis buffer (20 mM Tris, pH 8.0, 2 mM MgCl $2,250 \mathrm{mM} \mathrm{NaCl}, 10 \%$ (v/v) glycerol) and stored at $-80^{\circ} \mathrm{C}$. The cells were lyzed with an Emulsion flex in the presence of $4 \mathrm{mM}$ $\beta$-mercaptoethanol, $1 \mathrm{mM}$ PMSF and $1 \mu \mathrm{g} / \mathrm{ml}$ AP, LP and centrifuged for 1 hour with $100,000 \mathrm{~g}$ at $4{ }^{\circ} \mathrm{C}$. After addition of $10 \mathrm{mM}$ imidazol the supernatant was transferred to $0.5 \mathrm{ml} \mathrm{Ni-beads} \mathrm{per} \mathrm{liter} \mathrm{of} \mathrm{bacterial} \mathrm{culture} \mathrm{equilibrated} \mathrm{in} \mathrm{lysis} \mathrm{buffer} \mathrm{containing}$ protease inhibitors, washed with $10 \mathrm{mM}$ imidazol in S200 buffer (20 mM Tris, $\mathrm{pH} \mathrm{7.5,}$ $150 \mathrm{mM} \mathrm{NaCl}, 1 \mathrm{mM}$ DTT) and eluted with S200 buffer supplemented with $300 \mathrm{mM}$ imidazol. Protein-containing fractions were combined, concentrated and subjected to a Superdex 200 preparative gel filtration in S200 buffer. Fractions of importin ${ }^{\circledR}$ were dialyzed against MonoQ buffer (20 mM Tris, pH 7.5, $50 \mathrm{mM} \mathrm{NaCl}, 1 \mathrm{mM}$ DTT) and subsequently purified further by a MonoQ anion-exchange column. Proteins were eluted with a gradient of $50 \mathrm{mM}-500 \mathrm{mM} \mathrm{NaCl}$. After combining and concentrating importin $\AA$ fractions the solution was flash frozen and stored at $-80{ }^{\circ} \mathrm{C}$. 
Purified recombinant His-transportin (Baake et al. 2001), His-importin 5 (Jakel et al. 1998), His-importin 7 (Wohlwend et al. 2007), His-importin 9 (Muhlhausser et al. 2001) His-importin 13 (Jakel et al. 1998; Mingot et al. 2001) and His-CRM1 (Hutten et al. 2006) were kindly provided by Dr. habil. Ralph H. Kehlenbach, University of Göttingen, purified as described.

\subsection{Interaction experiments}

\section{Pull-down experiments}

For pulldown experiments with import receptors recombinant His-CFP-Aos1 or Uba2YFP-His were immobilized on cyanogenbromide-activated sepharose (Sigma Aldrich) according to the manufacturer's instructions, with final concentrations of $0.014 \mathrm{nmol}$ Aos 1 or $0.01 \mathrm{nmol}$ Uba2 per $1 \mu \mathrm{l}$ pure beads. $15 \mu \mathrm{l}$ beads were incubated for $1.5 \mathrm{~h}$ at $4{ }^{\circ} \mathrm{C}$ in $500 \mu \mathrm{l}$ pull-down buffer (50 mM Tris pH 7.4, $200 \mathrm{mM} \mathrm{NaCl}, 1 \mathrm{mM} \mathrm{MgCl}, 5 \%$ (v/v) glycerol) with $2 \mathrm{mg} / \mathrm{ml}$ BSA or ovalbumin and $0.05 \mathrm{nmol}$ recombinant import receptor. Where indicated, reactions contained $1 \mu \mathrm{M}$ RanQ69L loaded with GTP as described by Kehlenbach et al. (Kehlenbach et al. 1999). After three times washing with pulldown buffer, bound proteins were eluted with SDS-sample buffer and subjected to SDS-PAGE followed by silver staining.

Pulldown experiments with the export receptor CRM1 were carried out in $100 \mu \mathrm{l}$ pulldown buffer containing $20 \mu \mathrm{l}$ His-CRM1 immobilized on CNBr-activated sepharose beads with $0.5 \mu \mathrm{g}$ protein $/ \mathrm{ml}$ beads and $2.5 \mu \mathrm{g}$ His-Aos 1, $2.5 \mu \mathrm{g}$ His-Uba2 or $3 \mu \mathrm{g} \mathrm{His-Aos1/Uba2} \mathrm{cargo} \mathrm{in} \mathrm{the} \mathrm{absence} \mathrm{or} \mathrm{presence} \mathrm{of} \mathrm{GTP-loaded} \mathrm{RanQ69L}$ (Kehlenbach et al. 1999). Reactions with empty beads served as control for the detection of unspecific background binding of the tested cargo proteins to the beads.

\section{Analytical gel filtration}

Different combinations of $1 \mu \mathrm{M}$ purified recombinant His-CFP-Aos/Uba2-YFP-His complex (wt/wt, wt/mut, mut/wt, mut/mut) were incubated with $5 \mu \mathrm{M}$ of each recombinant importin $\alpha$ and importin $\beta$ in a total volume of $100 \mu$ transport buffer with $1 \mathrm{mM}$ DTT rotating for 1 hour at $4{ }^{\circ} \mathrm{C}$. Analytical gel filtration was performed with a Superose6-HR10/30 column in an Äkta purifier system (GE Healthcare). After equilibration of the column with 2 column volumes of transport buffer with $1 \mathrm{mM}$ DTT the sample was injected onto the column. The analytical filtration was performed with a flow of $0.2 \mathrm{ml} / \mathrm{min}$ and fractions of $0.3 \mathrm{ml}$ were collected. For evaluating the protein 
composition $50 \mu \mathrm{l}$ of every second fraction was loaded on a $5 \%$ - $20 \%$ gradient SDS gel and visualized by Coomassie-staining. Calculation of the corresponding elution profile from the Äkta (GE Healthcare) was done with sigma plot 8.02 (Systat Software Inc.).

\section{RanGAP1 assay}

Labeling of recombinant, purified Ran with [Y $\left.{ }^{32} \mathrm{P}\right] \mathrm{GTP}$ and RanGAP1 assays were essentially performed as described (Askjaer et al. 1999; Kehlenbach et al. 2001). The final reactions contained $1 \mu \mathrm{M}$ CRM1, $30 \mathrm{nM}\left[\mathrm{Y}^{32} \mathrm{P}\right]$ RanGTP , $60 \mathrm{nM}$ RanGAP1, 200 nM GTP and 3 or $5 \mu \mathrm{M}$ Aos1, Uba2 or E1 complex in a total volume of $50 \mu \mathrm{l} \mathrm{TB}$. Reactions with 1 or $3 \mu \mathrm{M}$ nuclear export sequence (NES; NS2 protein of minute virus of mice, CVDEMTKKFGTLTIHDTEK; Askjaer et al. 1999) served as positive controls. All components except for [ $\left.\mathrm{Y}^{32} \mathrm{P}\right] \mathrm{RanGTP}$ and RanGAP were mixed, after addition of $\left[Y^{32} \mathrm{P}\right]$ RanGTP and incubation for 10 minutes at room temperature RanGAP1 was added. 10 minutes after incubation at room temperature, the reaction was stopped with stop solution ( $7 \%$ charcoal, $10 \%$ ethanol, $0.1 \mathrm{M} \mathrm{HCl}, 10 \mathrm{mM} \mathrm{NaH}{ }_{2} \mathrm{PO}_{4}$ ), the charcoal was collected by centrifugation for 4 minutes at $14,000 \times \mathrm{g}$ and the released $\left[{ }^{32} \mathrm{P}\right]$ phosphate in the supernatant was measured by scintillation counting. Background counts from a reaction without RanGAP were subtracted and GTP hydrolysis was expressed as the percentage of the value of radioactivity measured in a reaction with RanGAP and without CRM1. A reaction with 1 or $3 \mu \mathrm{M}$ nuclear export signal (NES) served as positive control.

\subsection{Cell fractionation}

Cell fractionation by differential centrifugation

A 3 I culture of cycling HeLa suspension cells was harvested at a density of $5-8 x$ $10^{5} \mathrm{cells} / \mathrm{ml}$ by centrifugation with $70 \mathrm{xg}$ for 10 minutes at room temperature. The cells were washed two times with cold HBS (10 mM HEPES pH 7.4, $145 \mathrm{mM} \mathrm{NaCl}$ ), the resulting pellet was resuspended in 3.5 volumes of ice cold hypotonic lysis buffer (15 mM KCl, 1.5 mM MgOAc, 1 mM DTT, 10 mM HEPES-KOH pH 7.5, 1 mM PMSF, $1 \mu \mathrm{g} / \mathrm{ml}$ of each aprotinin, leupeptin, pepstatin) and then subjected to swelling on ice for 10 minutes. Subsequently the cells were lyzed with 10 strokes in a tight-fitting glass dounce homogenizer. Effective lysis was confirmed by microscopic analysis of $5 \mu \mathrm{l}$ cell suspension gently mixed with $5 \mu \mathrm{l}$ trypan blue revealing a blue staining for 


\begin{tabular}{|c|c|c|c|c|c|c|}
\hline$\underline{k \text {-factor }}$ & $\mathrm{g}_{\text {average }}$ & rpm & time [min] & $\underline{\mathrm{S}_{20}, \mathrm{w}}$ & organelle & fraction \\
\hline $42,639.3$ & 376 & 2,300 & 5 & $5 \times 10^{5}$ & $\begin{array}{l}>10^{7} \text { whole cells } \\
>10^{5} \text { intact nuclei }\end{array}$ & 1 \\
\hline $12,199.1$ & 1,313 & 4,300 & 10 & $7 \times 10^{4}$ & nuclear debris & 2 \\
\hline $4,119.1$ & 3,888 & 7,400 & 20 & $1 \times 10^{4}$ & $\begin{array}{c}>10^{4} \text { mitochondria, } \\
\text { lysosomes }\end{array}$ & 3 \\
\hline $1,219.5$ & 13,134 & 13,600 & 37 & $2 \times 10^{3}$ & $\begin{array}{c}>2 \times 10^{3} \text { lysosomes, } \\
\text { peroxisomes }\end{array}$ & 4 \\
\hline 122.0 & 131,294 & 43,000 & 15 & $5 \times 10^{2}$ & $\begin{array}{c}>5 \times 10^{2} \text { endoplasmic } \\
\text { reticulum }\end{array}$ & 5 \\
\hline 97.9 & 163,602 & $\begin{array}{r}48,000 \\
\text { SN }\end{array}$ & 55 & $1 \times 10^{2}$ & $\begin{array}{l}>10^{2} \text { polysomes } \\
\text { cytosol }\end{array}$ & $\begin{array}{c}6 \\
\mathrm{SN}\end{array}$ \\
\hline
\end{tabular}

Table 1: Centrifugation steps for fractionation of HeLa cells by differential centrifugation. Based on the S20,w values (Svedberg constants at $20^{\circ} \mathrm{C}$ in water) described by Sheeler Sheeler 1981 and chosen appropriate centrifugation times, the k-factors for the sedimentation of the according organelles were calculated according to $k$-factor $=S_{20, w} \cdot$ time [h]. Subsequently the corresponding speed for a Beckman Type 60 Ti rotor was calculated using Beckman's online rotor calculations tool.

most nuclei. The resulting homogenate was mixed with $1 / 5$ volume of ice cold hypertonic buffer (375 mM KCl, 22.5 mM MgOAc, 1 mM DTT, $220 \mathrm{mM} \mathrm{HEPES-KOH}$ $\mathrm{pH} 7.5,1 \mathrm{mM}$ PMSF, $1 \mu \mathrm{g} / \mathrm{ml}$ of each aprotinin, leupeptin, pepstatin) and centrifuged at $4{ }^{\circ} \mathrm{C}$ with 2,300 rpm in a Beckman Type $60 \mathrm{Ti}$ rotor for 5 minutes. The resulting supernatant was subjected to the next centrifugation step as listed in table 1 and this procedure was repeated until the last centrifugation step of 50,000 rpm for $1 \mathrm{~h}$. Calculations were carried out based on $S_{20, w}$ values (Svedberg-constants at $20^{\circ} \mathrm{C}$ in water) for different organelles as described by Sheeler (Sheeler 1981). Using the equation $k$-factor $=S_{20, w} \cdot$ time $[\mathrm{h}]$ and a chosen centrifugation time, $k$-factors for specific sedimentation of a certain organelle were calculated. The required centrifugation speed, expressed in rpm and gaverage were computed for the used rotor from the resulting $k$-factors by using Beckman's rotor calculations online tool (www.beckmancoulter.com/resourcecenter/labresources/centrifuges/rotorcalc.asp).

Resulting pellets from the different steps were resuspended in an appropriate volume of tranport buffer supplemented with $1 \mathrm{mg} / \mathrm{ml}$ of each aprotinin, leupeptin and pepstatin. Protein concentration of all samples, including the homogenate and the final supernatant, was determined and then adjusted to a final protein concentration 
of $1 \mathrm{mg} / \mathrm{ml}$ with $2 \times$ SDS sample buffer. $40 \mu \mathrm{l}$ of the resulting samples were loaded on a $5 \%$ - $20 \%$ gradient SDS gel and subsequently analyzed by immunoblotting.

Fractionation of murine hippocampal neurons

Samples of subcellular fractions of hippocampal neurons from mouse were kindly provided by Prof. Dr. Nils Brose, Max-Planck Institute for Experimental Medicine, Göttingen. The fractionations were prepared essentially as described previously (Jones et al. 1974) and designated as follows: H, homogenate; P1, nuclear pellet; S1, supernatant after nuclear sedimentation; P2, crude synaptosomal pellet; P3, light membrane pellet; S3, cytosolic fraction; LP1, heavy membrane pellet; LS1, intermediate fraction; SMP, synaptic plasma membranes; LP2, crude synaptic vesicle fraction; LS2, cytosolic synaptosomal fraction. $20 \mu \mathrm{g}$ of total protein per fraction were analyzed by SDS PAGE followed by immunoblotting.

\subsection{In vitro SUMOylation reaction}

\section{Detection via SDS-PAGE}

For analysis of $\mathrm{E} 1$ activity in in vitro SUMOylation reactions by SDS-PAGE, $79 \mathrm{nM}$ substrate (RanGAP1) was incubated with $225 \mathrm{nM}$ SUMO-1 $\triangle \mathrm{C} 4,27 \mathrm{nM}$ Ubc9 and 1.6 nM E1 in a total volume of $20 \mu \mathrm{SAB}$ buffer in the presence or absence of $1 \mathrm{mM}$ ATP at $30{ }^{\circ} \mathrm{C}$ for different periods of time. Reactions were stopped by addition of an equal volume $2 \times$ SDS sample buffer. $6 \mu$ of each sample was separated on a $5-20 \%$ gradient SDS gel and the substrate was subsequently detected by immunoblotting.

\section{Detection via FRET}

Fluorescence resonance energy transfer (FRET) based SUMOylation assays allows to measure kinetic, quantitative and high-throughput SUMOylation reactions (Bossis et al. 2005; Stankovic-Valentin et al. 2009). Briefly, the assay is set up as a standard in vitro SUMOylation reaction using a CFP-tagged substrate and YFP-tagged SUMO. If the CFP-substrate is modified with YFP-SUMO, the proximity of the fluorescent molecules is often close enough (less than $10 \mathrm{~nm}$ ) to enable efficient energy transfer from the excited CFP to YFP and thereby lowering the detected emission of CFP and enhancing the detected YFP emission. Therefore, the ratio between YFP emission $(527 \mathrm{~nm})$ / CFP emission $(485 \mathrm{~nm})$ increases. 
For the purpose of comparing kinetics of different concentrations of E1 enzymes, 100 nM CFP-RanGAPtail, $100 \mathrm{nM}$ YFP-SUMO and $44 \mathrm{nM}$ Ubc9 were incubated with 22 $\mathrm{nM}, 1.4 \mathrm{nM}$ or $86 \mathrm{pM} \mathrm{E1}$ in SAB buffer in a final reaction volume of $25 \mu \mathrm{l}$ in a 384-well fluorescence microtiter plate. The reaction was preincubated in a microplate fluorometer (Fluoroskan Ascent from Thermo Scientific) to reach the desired reaction temperature of $30^{\circ} \mathrm{C}$ and subsequently started by automatical addition of $5 \mu \mathrm{l}$ of 5 mM ATP. Simultaneous analysis of the fluoresecent signals was performed with a $430 \mathrm{~nm}$ excitation filter and $485 \mathrm{~nm}$ and $527 \mathrm{~nm}$ emission filters.

\subsection{Affinity purification of polyclonal antibodies}

The goat sera of polyclonal $\alpha$-Aos 1 and $\alpha$-Uba2 antibodies, raised against Histagged human full-length proteins, were available in the lab. Prior to use these antibodies had to be affinity purified. After depletion of the serum from antibodies against the His-tag by pre-adsorption against an unrelated immobilized His-tagged protein, antibodies were affinity purified from the serum using affinity columns containing the corresponding antigens. Briefly, the recombinant protein was extensively dialyzed against carbonate buffer $\left(0.2 \mathrm{M} \mathrm{NaHCO}_{3} \mathrm{pH} 8.9\right)$ overnight with at least two buffer changes. $\mathrm{CNBr}$ beads (Sigma) were prepared according to the manufacturer's instructions and incubated for $4 \mathrm{~h}$ at room temperature with the respective amount of protein to reach a final concentration of approximately $1 \mathrm{mg}$ protein $/ \mathrm{ml}$ beads. To determine the efficiency of coupling, the $O D$ at $280 \mathrm{~nm}$ was checked before and after coupling. The beads were washed two times with carbonate buffer and incubated with $100 \mathrm{mM}$ ethanolamine for $1 \mathrm{~h}$ at room temperature to block all remaining coupling sites. After three washing steps with 500 $\mathrm{mM} \mathrm{NaCl}$ in PBS the beads were used for His-depletion and affinity purification of antibodies from the serum. For this, $1-2 \mathrm{ml}$ of the His-tag affinity matrix $(1 \mathrm{mg}$ protein per $1 \mathrm{ml}$ pure beads) was incubated with $25 \mathrm{ml}$ serum and $25 \mathrm{ml}$ PBS in a falcon tube rotating at $4{ }^{\circ} \mathrm{C}$ for $3 \mathrm{~h}$ and subsequently the resulting supernatant was incubated with $2.5 \mathrm{ml}$ of the antigen affinity matrix (1 $\mathrm{mg}$ protein per $1 \mathrm{ml}$ pure beads) rotating at $4{ }^{\circ} \mathrm{C}$ over night. The beads were transferred into a column and washed two times with at least $50 \mathrm{ml}$ PBS supplemented with $0.5 \mathrm{M} \mathrm{NaCl}$. Washing was continued until no more protein was detected in the flow through by spotting on nitrocellulose followed by ponceau staining. When the column was completely emptied by gravity flow, the elution buffer $(0.2 \mathrm{M}$ acetic acid pH 2.7, $500 \mathrm{mM} \mathrm{NaCl})$ 
was carefully applied onto the column and antibodies were eluted with approximately 10 column volumes. Fractions of $500 \mu \mathrm{l}$ were collected and the $\mathrm{pH}$ was immediately neutralized by addition of $100 \mu$ of $1 \mathrm{M}$ Tris base. The antibody content of the fractions was determined by $\mathrm{OD}_{280 \mathrm{~nm}}$ and positive fractions were combined and concentrated to approximately $1 \mathrm{mg} / \mathrm{ml}$. The buffer was changed to PBS, antibodies were mixed with 1 volume of $87 \%(\mathrm{v} / \mathrm{v})$ glycerol and stored in aliquots at $-20{ }^{\circ} \mathrm{C}$.

Before usage, purified antibodies were generally tested for detection of the recombinant antigen, the endogenous antigen in HeLa cell lysate and cross reactivity with other human proteins in an immunoblot of HeLa lysate. Finally the antibodies were titrated and tested in immunoblotting and immunofluorescence to determine appropriate dilutions for usage. Purification using the described protocol yielded in approximately $1.2 \mathrm{mg} \alpha$-Aos 1 or $0.7 \mathrm{mg} \alpha$-Uba2 per $20 \mathrm{ml}$ of serum from final blood.

\section{Cell biology methods for mammalian cells}

\subsection{Cultivation of adherent and suspension cells}

Adherent HeLa cells were cultured in Dulbecco's modified Eagle Medium (DMEM, Gibco) supplemented with $10 \%$ (v/v) FBS, maintained in a humidified incubator with $5 \%(\mathrm{v} / \mathrm{v}) \mathrm{CO}_{2}$ at $37^{\circ} \mathrm{C}$. In general, cells were split at a $1 / 10$ ratio just before reaching confluency. For this purpose the cells were washed with sterile PBS, detached from the culture dishes with trypsin/EDTA and diluted with fresh medium to the designated confluency.

HeLa suspension cells were propagated in Jokliks medium,10 mM HEPES, pH 7.1 and $24 \mathrm{mM} \mathrm{NaHCO}_{3}$, supplemented with $5 \%$ (v/v) NCS, $5 \%(\mathrm{v} / \mathrm{v})$ FBS and, if the medium was older than two weeks, $2 \mathrm{mM}$ glutamine. The cells were cultured in spinner flasks at $100 \mathrm{rpm}$ at $37{ }^{\circ} \mathrm{C}$ at $3-10 \times 10^{5}$ cells $/ \mathrm{ml}$. The cell density was adjusted daily after determining the density using a Neubauer counting chamber.

For long term storage, exponentially growing cells were trypsinized and diluted into serum-containing medium to inactivate the protease. The cells were collected by centrifugation for 5 minutes at room temperature with $70 \times \mathrm{g}$, resuspended in FBS or NCS in case of suspension cells and were then supplemented dropwise with $7-10$ $\%(\mathrm{v} / \mathrm{v})$ DMSO under gentle agitation. Aliquots were slowly frozen at $-80{ }^{\circ} \mathrm{C}$ in box insulated with 2-propanol and for long-term storage transferred to liquid nitrogen tanks. 


\subsection{Transient transfection}

Adherent HeLa cells were seeded in 24-well plates 24 hours prior to transfection. Transient transfection was usually performed with $0.75 \mu \mathrm{g}$ DNA of the designated expression construct or of the corresponding empty vector using Polyfect transfection reagent (Qiagen) according to the manufacturer's instructions. Analysis of expression was carried out with fluorescence microscopy after fixation and processing as described for general detection of fluorescent proteins.

\subsection{Fluorescence based detection of intracellular proteins}

Overexpression and detection of fluorescent proteins

HeLa cells were seeded in 24-well plates on autoclaved $12 \mathrm{~mm}$ glass coverslips 24 hours prior to transfection. Transient chemical transfection was performed with 0.75 $\mu \mathrm{g}$ DNA of each expression construct using Polyfect transfection reagent (Qiagen) according to the manufacturer's instructions. 24 hours after transfection cells were washed three times with PBS to remove the medium. Cells were generally fixed with $3.7 \%(\mathrm{v} / \mathrm{v})$ formaldehyde in phosphate buffered saline (PBS) for 15 minutes, subjected to two washing steps in PBS and were then mounted onto glass slides with fluorescent mounting medium (Dako). If not stated differently, fluorescence was analyzed by confocal microscopy using a confocal LSM 510meta microscope (Zeiss) with a LCl Plan-Neofluar 63x/1.3 Imm Korr DIC objective and appropriate filter settings.

\section{Indirect immunofluorescence on cells}

HeLa cells grown on $12 \mathrm{~mm}$ glass coverslips were fixed for 15 minutes in $3.7 \%(\mathrm{v} / \mathrm{v})$ paraformaldehyde in PBS, washed with PBS and permeabilized for 5 minutes with icecold $0.2 \%(\mathrm{v} / \mathrm{v})$ Triton X-100 in PBS. After washing, cells were blocked with $2 \%$ $(w / w)$ BSA in PBS for 30 minutes. The coverslips were incubated for one hour at room temperature in 1:1,000 diluted affinity-purified anti-Aos1- or anti-Uba2 antibody from goat (approximately $0.5 \mu \mathrm{g} / \mathrm{ml}$ final concentration) in $2 \%(\mathrm{w} / \mathrm{v}) \mathrm{BSA} / \mathrm{PBS}$, washed with PBS and then incubated in a 1:1,000 dilution of Alexa488-coupled donkey anti-goat-antibody ( $2 \mu \mathrm{g} / \mathrm{ml}$ final concentration) (Molecular Probes). After extensive washing with PBS the cells were mounted and analyzed as described for general detection of fluorescent proteins. 


\section{Immunohistochemistry}

Staining of endogenous Uba2 in paraformaldehyde-fixed and paraffin-embedded slides of normal murine brain (Abcam). The manufacturer cut the tissue section to a thickness of $5 \mu \mathrm{m}$, mounted them on positively charged glass slides and stained one slide of each lot by haemotoxylin and eosin to ensure quality.

Slides were deparaffinized by incubating them twice for 10 minutes in xylene (Sigma), followed by hydration in $100 \%, 95 \%, 85 \%$ and $75 \%(\mathrm{v} / \mathrm{v})$ ethanol in distilled water for 5 minutes each. After transfer to pure distilled water for 5 minutes, slides were incubated in TBS ( $50 \mathrm{mM}$ Tris $\mathrm{pH} 7.6,100 \mathrm{mM} \mathrm{NaCl}$ ) three times for 5 minutes. For antigen retrieval, slides were boiled in $10 \mathrm{mM} \mathrm{Na}$-citrate $\mathrm{pH} 6.0$ supplemented with $0.05 \%(\mathrm{v} / \mathrm{v})$ Tween20 for 30 minutes in a microwave, cooled down to room temperature and transferred first into distilled water and then into TBS, three times for 5 minutes each. After 30 minutes blocking with $3 \%(w / v)$ BSA in TBS at room temperature, the tissue section on the slide was covered with a 1:20 dilution of affinity purified goat anti-Uba2 antibody (25 $\mu \mathrm{g} / \mathrm{ml}$ final concentration) in $1 \%(\mathrm{w} / \mathrm{v})$ BSA/TBS and incubated for 1 hour at room temperature. The slides were washed twice for 5 minutes with TBS and then stained for 1 hour at room temperature in the dark with $1 \%(\mathrm{w} / \mathrm{v})$ BSA/TBS containing 1:100 diluted Alexa488-coupled donkey anti-goat (Molecular Probes) and $0.25 \mu \mathrm{g} / \mathrm{ml}$ Hoechst33342. After extensive washing 3 times for 5 minutes with TBS, the tissue slides were mounted onto glass slides with $20 \mu \mathrm{l}$ SLOW-FADE Gold mounting medium (Invitrogen) and fluorescent signals were detected with a CellObserver (Zeiss) using Colibri LED, Plan-Apochromat 20x/0,8 objective and appropriate filters.

\subsection{In vitro import assay}

Import reactions were performed based on the method established by Adam et al. (Adam et al. 1990). HeLa cells grown on sterile $12 \mathrm{~mm}$ glass coverslips to $40-70 \%$ confluency were washed twice for 5 min on ice with transport buffer $(20 \mathrm{mM}$ Hepes $\mathrm{pH}$ 7,3, $110 \mathrm{mM} \mathrm{KOAc}, 2 \mathrm{mM} \mathrm{Mg}(\mathrm{OAc})_{2}, 1 \mathrm{mM}$ EDTA) supplemented with $1 \mathrm{mg} / \mathrm{ml}$ AP, LP and $2 \mathrm{mM}$ DTT. The plasmamembrane was permeabilized with $0.01 \%(\mathrm{w} / \mathrm{v})$ digitonin in transport buffer on ice for 4 minutes. Permeabilized cells were washed three times for 5 minutes and were then incubated for 45 minutes at $37^{\circ} \mathrm{C}$ with $30 \mu \mathrm{l}$ of a transport reaction mix containing fluorescently labeled cargo protein $(5 \mu \mathrm{M}$ of 
single E1 subunits or $1 \mu \mathrm{M}$ of E1 complexes), $15 \mu \mathrm{l}$ of cytosolic HeLa extract in transport buffer and either $1.5 \mu \mathrm{l}$ of an energy-regenerating system (final concentrations $1 \mathrm{mM}$ ATP, $5 \mathrm{mM}$ creatinphosphate and $100 \mathrm{U} / \mathrm{ml}$ creatine phosphate kinase) or as a specificity control with $2 \mu$ l energy-depleting system (final concentration $16 \mathrm{U}$ hexokinase and $5 \mathrm{mM}$ glucose in TB). Import assays with recombinant import receptors were performed with reaction mixes containing $1 \mu \mathrm{M}$ cargo protein (single E1 subunits or formed E1 complex), $1.5 \mu \mathrm{M}$ importin $\alpha$ and/or 1 $\mu \mathrm{M}$ importin $\beta$ and either $12 \mu \mathrm{M}$ Ran-GTP or RanQ69L-GTP, loaded with GTP as previously described (Kehlenbach et al. 1999). After the import reactions, cells were washed three times for 5 minutes with TB and were subsequently fixed and processed as described for general detection of fluorescent proteins.

\section{Preparation of cytosolic HeLa extract}

Cycling HeLa suspension cells were harvested at a density of $5-8 \times 10^{5}$ cells $/ \mathrm{ml}$ by centrifugation at $250 \times \mathrm{g}$ for 10 minutes at $4{ }^{\circ} \mathrm{C}$ in a Beckman JS-5.2 rotor. After washing two times with ice cold PBS and once with ice cold washing buffer $(10 \mathrm{mM}$ HEPES $\mathrm{pH} 7.3,110 \mathrm{mM} \mathrm{KOAc}, 2 \mathrm{mM} \mathrm{Mg}(\mathrm{OAc})_{2}, 2 \mathrm{mM}$ DTT), the cell pellet was resuspended in an equal volume of hypotonic lysis buffer (5 mM HEPES pH 7.3, 10 $\mathrm{mM} \mathrm{KOAc}, 2 \mathrm{mM} \mathrm{Mg}(\mathrm{OAc})_{2}$, $2 \mathrm{mM}$ DTT, $1 \mathrm{mM}$ PMSF, $1 \mu \mathrm{g} / \mathrm{ml}$ of each leupeptin, pepstatin, aprotinin) and then subjected to swelling on ice for 10 minutes. The cells were lyzed by 5 strokes in a tight-fitting stainless steel dounce homogenizer and the resulting homogenate was centrifuged at $1,500 \times \mathrm{g}$ for 15 minutes at $4{ }^{\circ} \mathrm{C}$ to remove cell debris and nuclei. The supernatant was first centrifuged at $15,000 \times \mathrm{g}$ for 20 minutes in a Beckman JA-20 rotor and subsequently at 100,000 $\mathrm{xg}$ for 1 hour in a Beckman $70.1 \mathrm{Ti}$ rotor. The final supernatant was dialyzed for 3 hours against 3 changes of transport buffer, concentrated to $10 \mathrm{mg} / \mathrm{ml}$ and aliquots were flash frozen in liquid nitrogen prior to storage at $-80^{\circ} \mathrm{C}$.

\subsection{Microinjection into adherent HeLa cells}

HeLa cells were grown on autoclaved $12 \mathrm{~mm}$ coverslides and medium was changed from DMEM to $\mathrm{CO}_{2}$-independent medium (Gibco) supplemented with $4 \mathrm{mM} \mathrm{L}$ glutamine 1 hour prior to injection. Microinjection mixes were prepared as follows: microinjection of pre-assembled E1 complexes was performed with mixtures of different combinations (wt/wt, wt/mut, mut/wt or mut/mut) of at least $1.4(-2.4) \mu \mathrm{M}$ 
purified recombinant His-CFP-Aos1/Uba2-YFP-His complex and $1.5 \mu \mathrm{M}$ Dextrancoupled TRITC, whereas injection mixtures of single subunits contained $4.5 \mu \mathrm{M}$ TRITC-Dextran and either $17 \mu \mathrm{M}$ His-CFP-Aos1 or $8 \mu \mathrm{M}$ Uba2-YFP-His. Inhibition experiments with monoclonal anti-Importin ${ }^{\circledR}$ antibody clone 3E9 (Abcam) were carried out with $2: 1: 1$ mixtures of antibody, TRITC-Dextran (final $1.6 \mu \mathrm{M}$ ) and either His-CFP-Aos1 (final $5.6 \mu \mathrm{M}$ ), Uba2-YFP-His (final $3.3 \mu \mathrm{M}$ ), YFP-M9 (final 8.3 $\mu \mathrm{M}$ ) or pre-assembled His-CFP-Aos1/Uba2-YFP-His (final $3 \mu \mathrm{M}$ ). After microinjecting cells using an eppendorf femtojet at room temperatur for approximately $20-30$ minutes, cells were washed with PBS and incubated for 30 minutes at $37^{\circ} \mathrm{C}$ and $5 \%$ $\mathrm{CO}_{2}$ atmosphere in DMEM (Gibco) supplemented with $10 \%(\mathrm{v} / \mathrm{v})$ fetal bovine serum. After extensive washing with PBS the cells were fixed and processed as described for general detection of fluorescent proteins.

\subsection{Analysis of nucleocytoplasmic shuttling}

Interspecies heterokaryon assay

Heterokaryon assays were performed essentially as described (Roth et al. 1998). Briefly, HeLa cells, seeded in 24-well plates, were transfected with the fluorescent expression construct pcDNA3.1(-)-CFP-Aos1 48 hours prior to the experiment. 24 hours before the assay, HeLa cells were mixed in a 2:3 ration with mouse 3 T 3 cells and seeded in one well of a 6 -well plate containing $4-5$ glass coverslips. The cells were subjected to medium supplemented with $100 \mu \mathrm{g} / \mathrm{ml}$ cycloheximide in order to inhibit protein biosynthesis and were incubated for 3 hours. The cells were then washed two times with PBS and fused in 50 \% (v/v) PEG2000 in PBS for 3 minutes at room temperature. PEG was washed away by three wash steps with PBS and subsequently the cells were incubated in cycloheximide-containing medium. Samples were taken at different time points and processed and analyzed as described for detection of fluorescent proteins. As de novo protein biosynthesis was inhibited by the addition of cycloheximide any fluorescent signal in the blue spotted mouse nuclei would indicate shuttling of CFP-Aos1.

\section{Combined FRAP and FLIP in multinuclear cells}

Nucleocytoplasmic shuttling was analyzed performing combined FRAP (fluorescence recovery after photobleaching) and FLIP (fluorescence loss in photobleaching) experiments in multinuclear cells with modifications based on the described 
techniques (Koster et al. 2005; Belaya et al. 2006). HeLa cells were grown in 4-well Lab-Tek $^{\mathrm{TM}}$ II coverglass chambers and transfected with the fluorescent expression construct pcDNA3.1(-)-CFP-Aos1 24 hours prior to the experiment. The cells were washed with PBS and fused to homopolykaryons in $50 \%$ (v/v) PEG2000 in PBS for 3 minutes at room temperature. PEG was washed away by three wash steps with PBS and subsequently the cells were incubated in medium at $37{ }^{\circ} \mathrm{C}$ in the incubation chamber of the microscope. The experiments were performed using a confocal LSM 510meta microscope (Zeiss) with a LCl Plan-Neofluar 63x/1.3 Imm Korr DIC objective and appropriate filter settings. Due to a high mobility of nuclear CFP-Aos1, measured in preparatory experiments, the fluorescence in one nucleus was completely bleached by scanning an approximately $15 \mu \mathrm{m} \times 10 \mu \mathrm{m}$ ROI (region of interest) within this nucleus 300 times with $100 \%$ intensity of the $458 \mathrm{~nm}$ argon laser and a speed of $6.39 \mu \mathrm{m} / \mathrm{sec}$. Pictures were taken before and at different time points after bleaching the nucleus as generally described in Detection of fluorescent proteins. To circumvent bleaching of the fluorescence signal by repeated image acquisition, the laser intensity was adjusted to $10 \%$ and exposure time for detection was minimized resulting in low-resolution images. For data interpretation fluorescence intensities of different ROls were calculated in percentage of the intensity of ROI 1, located within the bleached nucleus, before bleaching. Changes of the fluorescence intensity in ROI 1 within the bleached nucleus was compared to the intensity in ROI 2, 3 and 4 within unbleached nuclei in the same cell and the cytoplasmic background signal measured in ROI 5. Furthermore, the slopes of the signals after bleaching were calculated for ROI 1, average of the ROls 2-4 and the cytoplasmic background to compare the speed of signal accumulation. A steeper slope of ROI 1 compared to ROIs 2-4 would indicate shuttling of the transporter protein CFP-Aos1.

\section{Yeast methods}

\subsection{Culture and storage of yeast strains}

Yeast cells from glycerol stocks were first streaked on plates with a sterile glass pipette. For growth of cells in liquid culture, a preculture was inoculated with cells from plates and incubated over nigth. The main culture was inoculated with the preculture considering the growth rate of the strain and the growth temperature, with 
an optimal growth temperature of wild type strains of $30^{\circ} \mathrm{C}$. Wild type strains were grown in YPD or SC-complete medium, whereas plasmid containing strains were selected for growth in SC medium lacking the amino acid or base whose synthesis was encoded by the selective marker on the plasmid. If yeast strains were manipulated by chromosomal integration of genes, the transformants were selected by growth on the respective selective medium.

Stocks for long term storage of yeast strains were obtained by transferring fresh cell material from plates into a sterile cryo-tube containing $1.5 \mathrm{ml} 15 \%(\mathrm{v} / \mathrm{v})$ glycerol and subsequent dispersion of the cells by vortexing. To avoid the sedimentation of cells, the mixture was flash-frozen in liquid nitrogen prior to storage at $-80^{\circ} \mathrm{C}$.

\subsection{Preparation and transformation of competent yeast cells}

The transformation of yeast cells was performed according to a protocol adapted from Schiebel and Rietz (Schiestl et al. 1989).

A $10 \mathrm{ml}$ preculture, which was grown over night at $30{ }^{\circ} \mathrm{C}$, was diluted to $\mathrm{OD}_{600 \mathrm{~nm}}$ of 0.1 in $50 \mathrm{ml}$ and grown to an $\mathrm{OD}_{600 \mathrm{~nm}}$ of $0.6-0.8$. The cells were harvested by centrifugation at 3,200 rpm for 2 minutes at room temperature followed by one wash with $25 \mathrm{ml}$ sterile $\mathrm{ddH}_{2} \mathrm{O}$ and one wash in $12.5 \mathrm{ml}$ LiSorb $(10 \mathrm{mM}$ Tris- $\mathrm{HCl}, \mathrm{pH}$ 8.0, 1 M sorbitol, $100 \mathrm{mM}$ LiOAc, $1 \mathrm{mM}$ EDTA). Once all residual LiSorb was removed by an additional centrifugation step, cells were resuspended in $300 \mu \mathrm{l}$ LiSorb and $50 \mu \mathrm{l}$ carrier DNA (Salmon Sperm, Invitrogen) that had been denatured at $95{ }^{\circ} \mathrm{C}$ for 5 minutes and cooled on ice. Competent yeast cells were used immediately or stored at $-80^{\circ} \mathrm{C}$ in $110 \mu$ laliquots.

For each transformation $50 \mu \mathrm{l}$ of competent cells, thawed at room temperature, were mixed with DNA and incubated at room temperature. After 15 minutes $300 \mu$ LiPEG (10 mM Tris-HCl, pH 8.0, 40 \% (v/v) PEG3350, 100 mM LiOAc, 1 mM EDTA) were added, the mixture was briefly vortexed and incubated for 15 minutes at room temperature. $30 \mu \mathrm{L}$ DSO was added and mixed immediately by vortexing. The cells were subjected to heat-shock by incubation in a water bath at $42{ }^{\circ} \mathrm{C}$ for 10 minutes. Finally, the cells were centrifuged at 3,200 rpm for 3 minutes, resuspended in $200 \mu \mathrm{l}$ PBS, plated onto selective plates and incubated at the appropriate temperature.

No recovery was necessary when auxotrophic markers were used. However, for chromosomal integration of a transformed deletion cassette and subsequent 
selection with nourseothricin cells were centrifuged after heat shock, resuspended in $1 \mathrm{ml} \mathrm{YPD}$ and incubated with shaking for 6 hours before plating.

For transformation of PCR cassettes $5 \mu$ of a standard cassette PCR reaction (Janke et al. 2004) was used with $50 \mu \mathrm{l}$ of competent cells, whereas for transformation of plasmids $1 \mu \mathrm{g}$ DNA was used.

\subsection{Processing of positive yeast transformants}

Positive transformants were picked with a sterile pipette tip and re-streaked on selective plates to isolate single colonies. Single colonies were then streaked as a patch onto a fresh plate and, in case of antibiotic selection, single colonies were again streaked on fresh selection plates to identify positive ones. For storage of newly generated yeast strains, glycerol stocks of two independent clones were prepared.

\subsection{Generation of yeast UBA2 shuffle strains}

For phenotypic analysis of the essential UBA2 gene (Dohmen et al. 1995), an UBA2 shuffle strain was constructed. In a shuffle strain the wild type copy of a gene is deleted and replaced with a marker, while cells are kept alive by another copy of this gene on an URA3 plasmid.

First, the original strain ESM356-1 was transformed with the URA3 plasmid containing the UBA2 gene including $500 \mathrm{bp}$ up- and downstream of the coding region (pRS316-UBA2), resulting in the yeast strain ESM356-1*/pRS316-UBA2. Subsequently, the genomic copy of the UBA2 gene was deleted and replaced by a cassette containing a resistance marker for the antibiotic nourseothricin (for details see Cassette PCR). Disruption of the genomic copy of the gene in the constructed shuffle strain ESM356-1*/pRS316-UBA2/uba2A::nat was confirmed by colony PCR (for details see Yeast colony PCR). Subsequently, constructs of the HIS3 plasmid p413 containing ORFs for differents Uba2 variants were transformed into the shuffle strain ESM356-1*/pRS316-UBA2/uba2A::nat. Single colonies of these transformants were characterized.

\section{Cassette PCR}

For deletion of the UBA2 gene in yeast the cassette module natNT2 was amplified with S1-/S2-primers, designed to anneal 500 bp up- and downstream of UBA2, using 
pKS134:pFA6y-natNT2 as template as described by Janke and Knop (Janke et al. 2004). A standard $50 \mu \mathrm{l}$ reaction contained $5 \mu \mathrm{l} 10$ x buffer 1 (500 mM Tris-HCl, pH 9.2, $160 \mathrm{mM}\left(\mathrm{NH}_{4}\right)_{2} \mathrm{SO}_{4}, 7.5 \mathrm{mM} \mathrm{MgCl} 2,2 \mathrm{mM}$ each dNTP), $10 \mu \mathrm{M}$ of each primer, $100 \mathrm{ng}$ cassette plasmid DNA and $2 \mathrm{U}$ Taq polymerase mixed with $0.4 \mathrm{U}$ Vent polymerase. Initial denaturation ( $97^{\circ} \mathrm{C}-5$ minutes) was followed by 10 cycles of denaturation $\left(97{ }^{\circ} \mathrm{C}-30\right.$ seconds), annealing $\left(52^{\circ} \mathrm{C}-30\right.$ seconds) and extension $\left(68{ }^{\circ} \mathrm{C}-1 \mathrm{~min} / \mathrm{kb}\right.$ ) and 20 cycles of denaturation $\left(97^{\circ} \mathrm{C}-30\right.$ seconds), annealing (52 ${ }^{\circ} \mathrm{C}-30$ seconds) and extension $\left(6{ }^{\circ} \mathrm{C}-1 \mathrm{~min} / \mathrm{kb}+20\right.$ ses/cycle). Products were purified after analysis on an agarose gel.

\section{Yeast colony PCR}

Colony PCR was used to directly confirm the disruption of the UBA2 gene in yeast. The 5'-primer was designed to anneal 112 bp upstream and the 3'-primer annealed $109 \mathrm{bp}$ downstream of the gene. A wildtype yeast strain was included as negative control. A small amount of yeast cells was resuspended in $50 \mu \mathrm{l} 0.01 \% \mathrm{~N}$-Lauroyl sarcosine, $0.02 \mathrm{~N} \mathrm{NaOH}$ and boiled for 10 minutes at $95{ }^{\circ} \mathrm{C}$. $1 \mu \mathrm{l}$ of the resulting extracts was used for a PCR reaction. The $25 \propto$ l reaction volume contained $2.5 \mu \mathrm{l}$ Taq polymerase buffer, $2.5 \mu \mathrm{l}$ dNTP-Mix (final $2 \mathrm{mM}$ each), $1.5 \mu \mathrm{l}$ of each primer (final $10 \mu \mathrm{M}$ ) and $1 \mu \mathrm{l}$ Taq polymerase $(1 \mathrm{U} / \mu \mathrm{l})$. Initial denaturation $\left(97{ }^{\circ} \mathrm{C}-5\right.$ minutes) was followed by 35 cycles of denaturation ( $97^{\circ} \mathrm{C}-30$ seconds), annealing $\left(52{ }^{\circ} \mathrm{C}-30\right.$ seconds) and extension $\left(68^{\circ} \mathrm{C}-1 \mathrm{~min} / \mathrm{kb}\right)$ and a final extension step $(68$ ${ }^{\circ} \mathrm{C}$ - 3 minutes). Products were analyzed on agarose gels.

\subsection{Phenotypic analysis of UBA2 shuffle strains}

For the phenotypic analysis of exogenous encoded Uba2 variants the transformed shuffle strains were subjected to selection on plates containing 5-FOA leading to the loss of the URA3 plasmid pRS316-UBA2 containing the wild type copy of the UBA2 gene and thereby selecting for cells with the protein encoded by the transformed overexpression construct. The URA3 gene encodes orotidine-5'phosphate decarboxylase, an enzyme required for the biosynthesis of uracil. URA3 cells convert 5-FOA into toxic 5-fluorouracil and thus URA3-positive cells die, whereas ura3negative cells are resistant to the drug. 
Intracellular localization of C-terminally GFP-tagged Uba2 variants was determined by fluorescence microscopy. For this, precultures inoculated with colonies from 5FOA plates and grown over night at $30{ }^{\circ} \mathrm{C}$ in filter-sterilized SC-complete medium were diluted to $\mathrm{OD}_{600 \mathrm{~nm}}$ of 0.05 and grown to an $\mathrm{OD}_{600 \mathrm{~nm}}$ of $0.3-0.5 .2 .5 \mathrm{ml}$ of the cultures were harvested by centrifugation at 3,200 rpm for 3 minutes at room temperature and the pellet was resuspended in $1 \mathrm{ml}$ of $3.7 \%(\mathrm{v} / \mathrm{v})$ PFA in PBS. After incubation for 10 minutes at room temperature the cells were spun down, $500 \mu$ PBS were added and the samples were kept on ice. DNA was stained with $1 \mathrm{ml}$ of $1 \mu \mathrm{g} / \mathrm{ml}$ Hoechst33342 in PBS for 1 hour at $4{ }^{\circ} \mathrm{C}$ in the dark. The cells were washed two times for 5 minutes with PBS and the resulting pellet was resuspended in $20 \mu \mathrm{l}$ antifade mounting medium (DakoCytomation). $3.5 \mu$ of the suspension were applied onto a glass slide and covered with a coverslip. Fluorescent signals were detected with a Cell Observer (Zeiss) using Colibri LED with appropriate filters and a PlanNeoFluar 100x/1.30 oil objective.

Growth test of UBA2 shuffle strains

Logarithmically growing cells were harvested and resuspended in PBS at a concentration of $1 \mathrm{OD}_{600 \mathrm{~nm}}$ per $\mathrm{ml}$ corresponding to approximately $2 \times 10^{7}$ cells $/ \mathrm{ml}$. Serial 1:5 dilutions in PBS were prepared and $2-3 \mu$ of each dilution was spotted onto plates and incubated at appropriate temperatures.

For analysis of rescue of UBA2 deletion by different Uba2 variants the same dilutions of cells were tested for growth on SC-His plates and 5-FOA plates in parallel. SC-His plates only selected for the presence of the p413 expression construct, thus allowing to compare the dilutions, whereas 5-FOA plates selected for cells without the UBA2 gene encoded in the pRS316 construct and thereby allowed to compare rescue efficiency of the overexpressed Uba2 variant.

Fractionation of UBA2 shuffle strains

A $500 \mathrm{ml}$ culture of logarithmically growing cells with $\mathrm{OD}_{600 \mathrm{~nm}}$ of $0.5-0.6$ was harvested by centrifugation at 3,200 rpm for 3 minutes and resuspended in spheroblasting buffer $(50 \mathrm{mM}$ Tris- $\mathrm{HCl}, \mathrm{pH} 7.5,10 \mathrm{mM} \mathrm{MgCl} 2,1 \mathrm{M}$ sorbitol, $1 \mathrm{mM}$ DTT). After 15 minutes incubation at room temperature the cells were harvested, resuspended in $15 \mathrm{ml}$ spheroblasting buffer supplemented with $0.75 \mathrm{mg} / \mathrm{ml}$ zymolyase $20 \mathrm{~T}$ and incubated for 2 hours at $30{ }^{\circ} \mathrm{C}$. Spheroblasts were spun down 
with 3,200 rpm for 5 minutes and all following steps were performed on ice or at $4{ }^{\circ} \mathrm{C}$. The suspension was washed 3 times with spheroblast buffer and the resulting pellet was resuspended in $5 \mathrm{ml}$ Ficoll buffer (18\% Ficoll-400, $10 \mathrm{mM}$ Tris- $\mathrm{HCl}, \mathrm{pH} 7.5,20$ $\mathrm{mM} \mathrm{KCl}, 5 \mathrm{mM} \mathrm{MgCl}$, $3 \mathrm{mM}$ DTT, $1 \mathrm{mM}$ EDTA, $0.1 \mathrm{mM} \mathrm{PMSF}, 1 \mu \mathrm{g} / \mathrm{ml}$ AP and LP, $25 \mathrm{mM} \mathrm{N}$-Ethylmaleimide (NEM), $25 \mathrm{mM}$ iodoacetamide, $50 \mathrm{mM} \mathrm{NaF}$ ). Cells were homogenized with approximately 50 strokes in a tight-fitting glass dounce homogenizer. After confirmation of efficient lysis with a light microscope the homogenate was centrifuged with 4,500 rpm for 5 minutes in a $5417 \mathrm{R}$ refrigerated microcentrifuge (Eppendorf) to remove non-lyzed cells and cell debris. The supernatant was transferred into fresh tubes and subjected to repeated centrifugation in fresh tubes unitl no further pellet formation was detected. The final centrifugation step with 14,000 rpm for 20 minutes separated the nuclear fraction in the pellet from the cytosolic fraction in the supernatant. The nuclear fraction was resuspended in $2 \mathrm{x}$ SDS sample buffer and proteins in the cytosolic fraction were enriched by TCA precipitation followed by resuspension in $4 \times$ SDS sample buffer. All samples were stored at $-80{ }^{\circ} \mathrm{C}$. For analysis of the fractions the protein concentration was determined in the SDS samples using reducing agent-compatible BCA Protein Assay (Pierce). Equivalent amounts of protein from the samples were analyzed by SDS PAGE and subsequent immunoblotting. 


\section{RESULTS}

\section{Generation and characterization of SUMO E1 variants}

A number of protocols in the literature describe the generation and purification of recombinant SUMO E1 enzyme (Johnson et al. 1997; Desterro et al. 1999; Gong et al. 1999; Okuma et al. 1999; Long et al. 2000; Pichler et al. 2002; Werner et al. 2009). Many of these protocols, including the standart protocol from our laboratory, are based on co-expression and subsequent co-purification of the two subunits Aos1 and Uba (Okuma et al. 1999; Long et al. 2000; Pichler et al. 2002). Since a number of experiments whithin this thesis required the reconstitution of E1 complexes containing different combinations of Aos1- and Uba2-variants with or without fluorescent tag I established a system in which single subunits are purified and active E1 complex is subsequently reconstituted, allowing to flexibly combine different variants of Aos1 and Uba2 (Werner et al. 2009).

\subsection{Reconstitution of $E 1$ complex from singly His-tagged subunits}

Preliminary data from our lab revealed that combination of His-Aos1 and His-Uba2 (N-terminally tagged) does not lead to a very active E1 complex (K. Chmielarska, $\mathrm{PhD}$ thesis), whereas previous studies based on recombinant SUMO E1 with Uba2His demonstrated that E1 composed of C-terminally tagged Uba2 does exhibit E1 activity (Johnson et al. 1997; Okuma et al. 1999; Long et al. 2000). Therefore I established a protocol in which E1 is reconstituted from N-terminally His-tagged Aos1 and C-terminally His-tagged Uba2 (Werner et al. 2009).

As shown in Figure 8A, His-Aos1 and Uba2-His were independently expressed from pET28a- or pET28b-based constructs and the recombinant proteins were purified via nickel-pull-down and gel filtration and, in the case of Uba2, also anion-exchange chromatography. After combination of purified His-Aos1 and Uba2-His, the assembled E1 complex was separated from excess of any of the single subunits by gel filtration. Comparison of E1 purified according to the co-expression protocol versus the new reconstitution protocol (Fig. 8B) demonstrated that both ways of E1 purification resulted in complexes of comparable purity. Due to a higher expression 
A

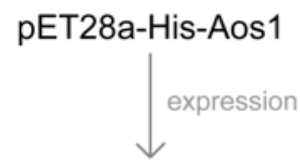

bacterial lysate

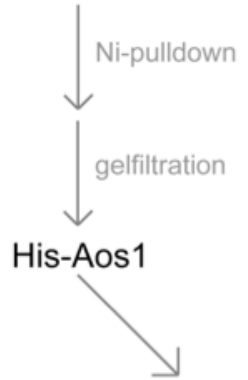

His-Aos1 / Uba2-His
B

pET28b-Uba2-His

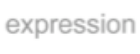

bacterial lysate

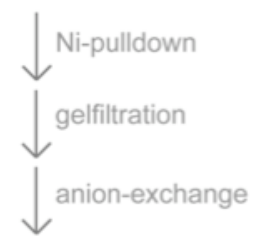

Uba2-His

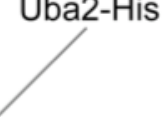

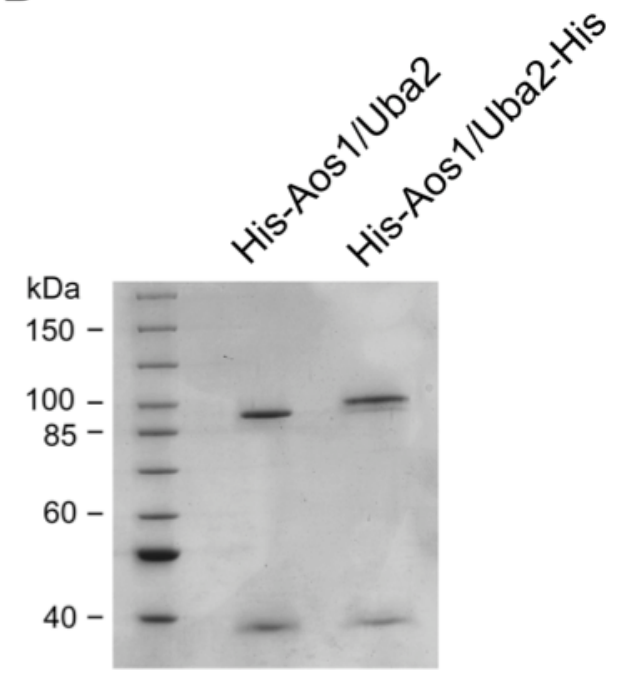

Figure 8: Purification of His-Aos1/Uba2-His. (A) Schematic illustration of E1 purification procedure according to the new reconstitution protocol. (B) Comparison of recombinant HisAos1/Uba2 and His-Aos1/Uba2-His purified according to the old co-expression protocol or the new reconstitution protocol respectively. The Coomassie stained SDS gel reveals identical purity of E1 complex by means of both purification procedures.

level of singly expressed Uba2-His compared to untagged Uba2 during coexpression, the amount of E1 obtained by the standard co-expression protocol of 0.5 - $1.5 \mathrm{mg}$ per liter $E$. coli culture was lower compared to $1.5-3 \mathrm{mg}$ Uba2-His and 5 $7 \mathrm{mg}$ His-Aos 1 per liter culture obtained by the new protocol.

\subsection{Generation of fluorescently labeled E1 complex}

Analysis of the intracellular localization of SUMO E1 complex required fluorescence microscopic detection of the single subunits within assembled E1 complexes. For this reason, the bacterial expression vectors $\mathrm{pET} 28 \mathrm{a}$ and $\mathrm{pET} 28 \mathrm{~b}$ were modified by insertion of ECFP or EYFP (PCR amplified from pECFP-C1 or pEYFP-C1) into the multiple cloning sites, resulting in expression vectors that coded for a $\mathrm{N}$-terminal HisCFP tag ( $p E T 28 a-E C F P)$ or a C-terminal YFP-His tag (pET28b-EYFP). Expression of Aos1 and Uba2 from these constructs allowed purification of His-CFP-Aos1 and Uba2-YFP-His and subsequent reconstitution of His-CFP-Aos1/Uba2-YFP-His complex. The expression and purifciation procedures were identical to HisAos1/Uba2-His and resulted in comparable amounts of equally pure E1 complex (demonstrated in Fig. 18). 


\subsection{Reconstituted His-Aos1/Uba2-His complex exhibits comparable} specific activity to co-purified His-Aos1/Uba2

In contrast to the commonly used E1 that contained His-tagged Aos 1 and untagged Uba2, E1 complex generated according to the new protocol included His-tagged Uba2. Since it has to date not been tested whether C-terminal tagging of Uba2 to some extent interferes with the catalytical E1 activity, I compared the E1 activities of recombinant purified His-Aos1/Uba2 and His-Aos1/Uba2-His in in vitro SUMOylation assays.

In vitro SUMOylation of RanGAP1 was set up with E1 activity as the rate-limiting factor of the reaction and the assay was performed with identical concentrations of His-Aos1/Uba2 or His-Aos1/Uba2-His (Fig. 8B) in parallel. Analysis by immunoblotting with $\alpha$-RanGAP1 antibody (Fig. 9A) revealed that both SUMOylation reactions proceeded with approximately similar kinetics. For a more detailed analysis, I turned to a FRET-based in vitro SUMOylation assay developed in our lab (Bossis et al. 2005). This assay involves YFP-tagged SUMO and CFP-tagged model substrate (RanGAP-tail). Upon conjugation FRET can occur between CFP and YFP, which can be followed online using a fluorescence plate reader, by measuring emission at 480 and $527 \mathrm{~nm}$ after excitation of CFP at $430 \mathrm{~nm}$. Figure 9B clearly shows that the reaction rates for different concentrations of E1 were very similar between His-Aos1/Uba2 and His-Aos1/Uba2-His.

Taken together, these data demonstrate that purification of Aos1/Uba2 according to

A

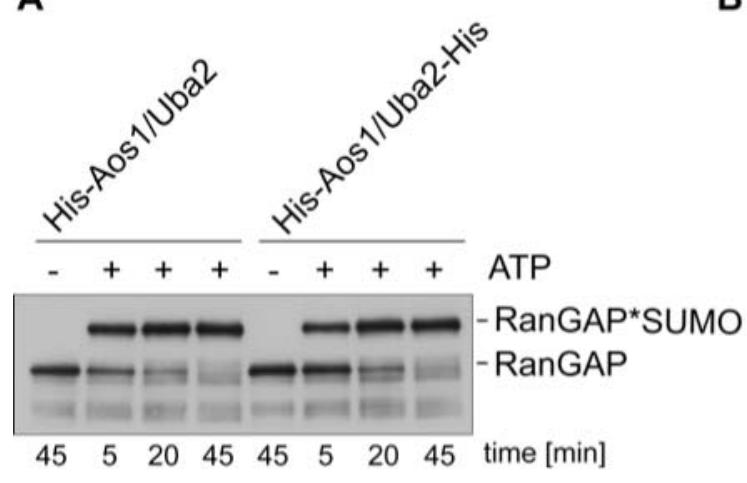

B

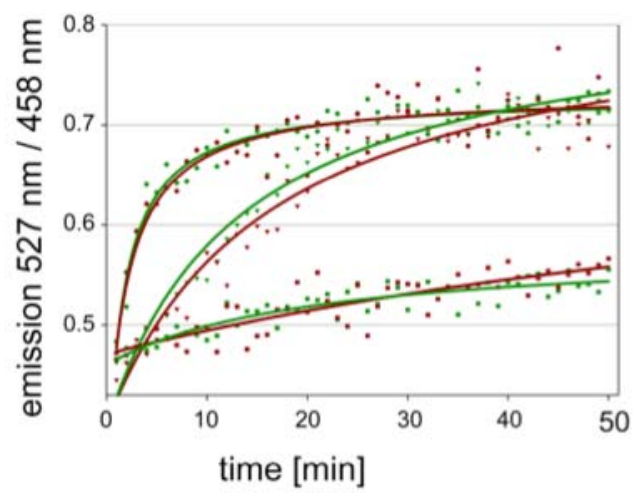

Figure 9: Identical E1 activities of His-Aos1/Uba2 and His-Aos1/Uba2-His. (A) In vitro SUMOylation of RanGAP with limiting amounts of E1 detected by SDS PAGE and $\alpha$-RanGAP immunoblotting. (B) In vitro SUMOylation reaction detected by FRET reveals identical kinetics for His-Aos1/Uba2-His (green) and His-Aos1/Uba2 (red) at the tested concentrations of $22 \mathrm{nM}$ (dots), $1.4 \mathrm{nN}$ (triangles) and $86 \mathrm{pM} \mathrm{E1}$ (quadrates) respectively. 
the new protocol results in E1 enzye of the same specific activity as obtained by the standard protocol based on co-expression.

\section{Characterization of the nuclear import of SUMO E1}

Based on the predominantly nuclear localization of Aos1 and Uba2 in cells (Dohmen et al. 1995; Azuma et al. 2001; Pichler et al. 2002) it is obvious that the proteins have to be imported into the nucleus after synthesis in the cytoplasm as well as after nuclear breakdown in mitosis. Since the mechanisms underlying the generation of active nuclear SUMO E1 are to date largely unknown, I performed a detailed characterization of the nuclear import of Aos1/Uba2.

\subsection{Aos1 and Uba2 contain distinct functional NLSs}

To gain insights into the import mechanisms of Aos1/Uba2, I first addressed the question whether import of the single subunits can occur independently of complex formation. For the Uba2 homolog from Saccharomyces cerevisiae it has previously been shown by overexpression of a C-terminally GFP-tagged deletion fragment of the protein that the C-terminal region (amino acids 551 - 636) is necessary for nuclear localization (Dohmen et al. 1995). Sequence alignments of the C-termini of Uba2 from different species (Fig. 10) revealed a cluster of basic amino acids

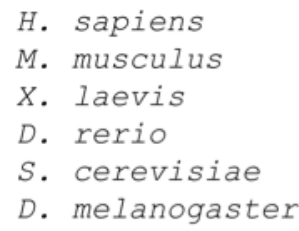

DVEFEVVGDAPEKVGPKQAEDA-AKSITNGSDDGAQPSTS--TAQEQDDVLIVDSDEEDS 57 DVEFEVVGDSPEKVGPKQAEDA-AKSIANGSDDGAQPSTS--TAQEQDDVLIVDSDEEGP 57 ---FEVVGDVPEKGPQKPPEES-VKNITNGSDDGAQPSTS--KAQDQDDVLIVDSDEESP 54 -----VVGDAPDKAPAPSAPEE-GKNIANGNKDSAQPSTSSKAAVEDDDVLLVDSDEEPS 54 ---CNTCSLPDVEVPLIKANNSPSKNEEEEKNEKGADVVATTNSHGKDGIVILD-DDEGE 56 -------DDGPSTSKRSRPNEVVEEDDDDCLVIEEDEDQADVVVVATDKLSVQSPPKSGS 53

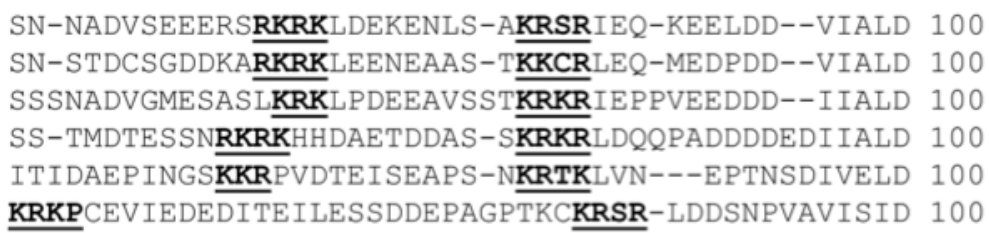

Figure 10: Sequence alignment of the C-terminus of Uba2. The C-terminal 100 amino acids of Uba2 from $H$. sapiens, $M$. musculus, $D$. rerio, $X$. laevis, $D$. melanogaster and $S$. cerevisiae were aligned using ClustalW2. Conserved clusters of basic amino acids potentially participating in nuclear import are underlined and highlighted in bold. 

H. sapiens
M. musculus
D. rerio
$X$. laevis
D. melanogaster
S. cerevisiae

H. sapiens

M. musculus

D. rerio

$X$. laevis

D. melanogaster

S. cerevisiae

H. sapiens

M. musculus

D. rerio

$X$. laevis

D. melanogaster

S. cerevisiae

DIEKKPESFFTQFDAVCLTCCSRDVIVKVDQICHKNSIKFFTGDVFGYHGYTFANLG-EH 172 DVEKKPESFFTKFDAVCLTCCSRDVI IKVDQICHRNSIKFFTGDVFGYHGYTFANLG-EH 176 PVESKPDDFFFQFDAVCLTRCSRDLMVRVDQLCASRNIKVFCGDVYGYNGYMFSDLGQEY 174 NINQKSDDFFTQFDVVCLTSCPSDLLVRVNHICHKHNIKFFTGDVYGYHGSMFADLG-EH 170 PLKEKTSEFFGQFDVVVVNGATNEELLRIDTICRDLGVKFIATDVWGTFGFYFASLQ-KH 175 DLQEKDEEFFQQFDLVVATEMQIDEAIKINTLTRKLNIPLYVAGSNGLFAYVFIDLI--- 167

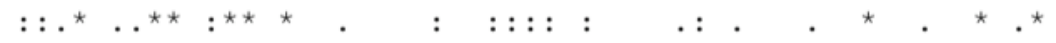

EFVEEKTKVAKVSQGVEDGPDTKRAKLDSSE----------TTMVKKKVVFCPVKEALEV 222 EFVEEKTKVAKVSQGVEDGPEAKRAKLDSSE----------TTMVKKKVLFCPVKEALEV 226 HYVEEKPKVVKGSNEANDGPEAKKPKIDPNE--------TTMVKKTISFCSLKEALEV 224 EFVEEKAKVTKAKPLVEDGPEA KKAKIDPTE---------TILVKKKVQFCPLKDALEI 220 SYVEDVINHKVVAN-------SEKKKKYETV----------SIPTQRDVDYPGYSAWLDF 218 EFISEDEKLQSVRPTTVGPISSNRSIIEVTTRKDEEDEKKTYERIKTKNCYRPLNEVLST 227 $::: \quad: \quad::$ :

DWSSEKAKAALKRTTSDYFLLQVLLKFRTDKGRDPSSDTYEEDSELLLQIRNDVLDSLGI 282 DWSGEKAKAALKRTAPDYFLLQVLLKFRTDKGRDPTSESYKEDAELLLQIRNDVFDSLGI 286 DWTTEKAKSSLKRI PADYFLLQVLLKFRTDKGRDPQPDSFAEDSQLLLQIRDDVLETMGL 284 DWRSEKAKSALKKTPTDYFLLQVLMKFRTDKGRDPQPSSYQEDSELLLQICSDVLDSLGV 280 DVTEPSYLRKLKRNGPGVLLLSVLQKFRTTHKRDPSYKTREADLELLRGIRDELLPNS-- 276 ATLKEKMTQRQLKR-VTSILPLTLSILQYDLNQKGKAISFEQMKRDAAVWCENLGVPATV 286

Figure 11: Sequence alignment of Aos1. Amino acid sequences of Aos1 from $H$. sapiens, $M$. musculus, D. rerio, $X$. laevis, D. melanogaster and S. cerevisiae were aligned using ClustalW2. Conserved clusters of basic amino acids potentially participating in nuclear import are underlined and highlighted in bold. This figure shows the section from amino acid 114 to 282 of of the alignment, for the complete alignment see Supplemantal Fig.1

conserved from human to fly that were reminiscent of a classical bipartite NLS. Before I started my investigations there was no evidence for a functional NLS in the E1 subunit Aos1. However, an alignment of Aos1 homologs from different species (Fig. 11) revealed two conserved clusters of basic amino acids matching the characteristics of classical NLSs.

To test whether these basic sequences are required for nuclear import I introduced mutations substituting amino acids of the potential NLSs by alanines (Fig. 12A) and compared the localization of wild type and mutant variants of CFP-Aos1 and Uba2YFP upon transient transfection of HeLa cells (Fig. 12B). The analysis of Aos1 revealed that CFP-Aos1-KRAK195-198A 4 but not CFP-Aos1-KKKV207-210A 4 localized to the cytoplasm of transfected cells (Fig. 12B, upper panel). This result showed that only the first of the two basic clusters participates in mediating nuclear import of Aos1 and pointed towards a monopartite and not a bipartite classical NLS. Interestingly, a more detailed comparison with identified import sequences of other proteins revealed a striking similarity of the identified region of the Aos1 NLS with the conserved residues of the NLS of the oncoprotein c-Myc $\left({ }^{320} \mathrm{PxxKRxKLD}^{328}\right)$ (Stone et al. 1987; Dang et al. 1988). Since all required residues of the c-Myc NLS are found 

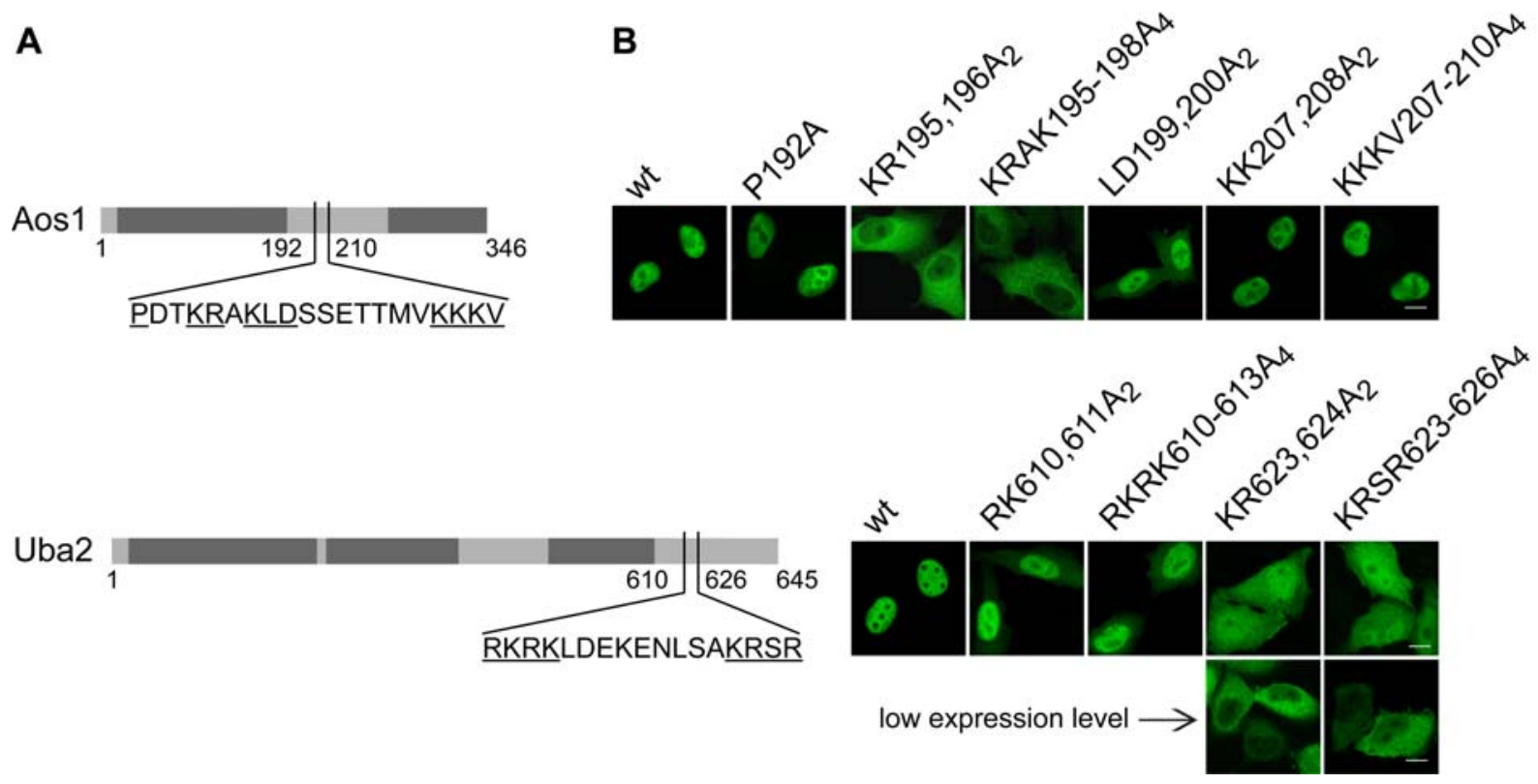

Figure 12: Both Aos1 and Uba2 contain distinct NLSs. (A) Schematic illustration of potential NLSs in Aos1 or Uba2. Amino acids changed by alanines for analysis of their participation in nuclear import are underlined. (B) HeLa cells were transiently transfected with pET28a-CFP-Aos1 (upper panel) or pET28b-Uba2-YFP (lower panel). 24 hours post transfection intracellular localization of overexpressed wild type and variant CFP-Aos1 or Uba2-YFP was detected by fluorescence microscopy. Localization of Uba2 variants upon low expression levels is indicated. Bar, $10 \mu \mathrm{m}$.

within Aos1 (Conti et al. 2000), I additionally performed a mutational analysis to verify the importance of these residues in mediating nuclear import of Aos1. Figure 12B demonstrates that Aos1-LD199,200A 2 partially mislocalized to the cytoplasm, whereas substitution of the proline did not alter the localization. Hence, the identified NLS of Aos 1 consists of a cluster of basic residues directly followed by leucine and aspartate and thereby largely matches the conserved amino acids $\left({ }^{320} \mathrm{PxxKRxKLD}{ }^{328}\right)$ of the unconventional NLS of c-Myc (Stone et al. 1987; Dang et al. 1988; Makkerh et al. 1996).

Mutational analysis of Uba2-YFP revealed an important role of two clusters of basic amino aicds, RKRK610-613 and KRSR623-626, in mediating nuclear import (Fig. $12 \mathrm{~B}$, lower panel). While replacement of the first cluster by alanines resulted in only partial mislocalization of Uba2, Uba2-KRSR623-626A 4 showed a strong cytoplasmic accumulation. The observed effect was most apparent in cells expressing lower levels of the protein. Thus, these results confirm the suggestion by Dohmen et al. that a basic cluster of amino acids in the C-terminal region of Uba2 participates in nuclear import (Dohmen et al. 1995). However, whether the import sequence of Uba2 is a bipartite cNLS or contains two overlapping monopartite NLSs could not be conclusively clarified. 
A
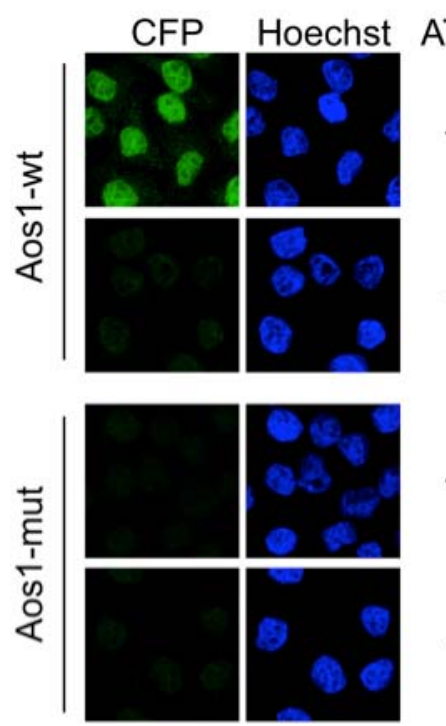
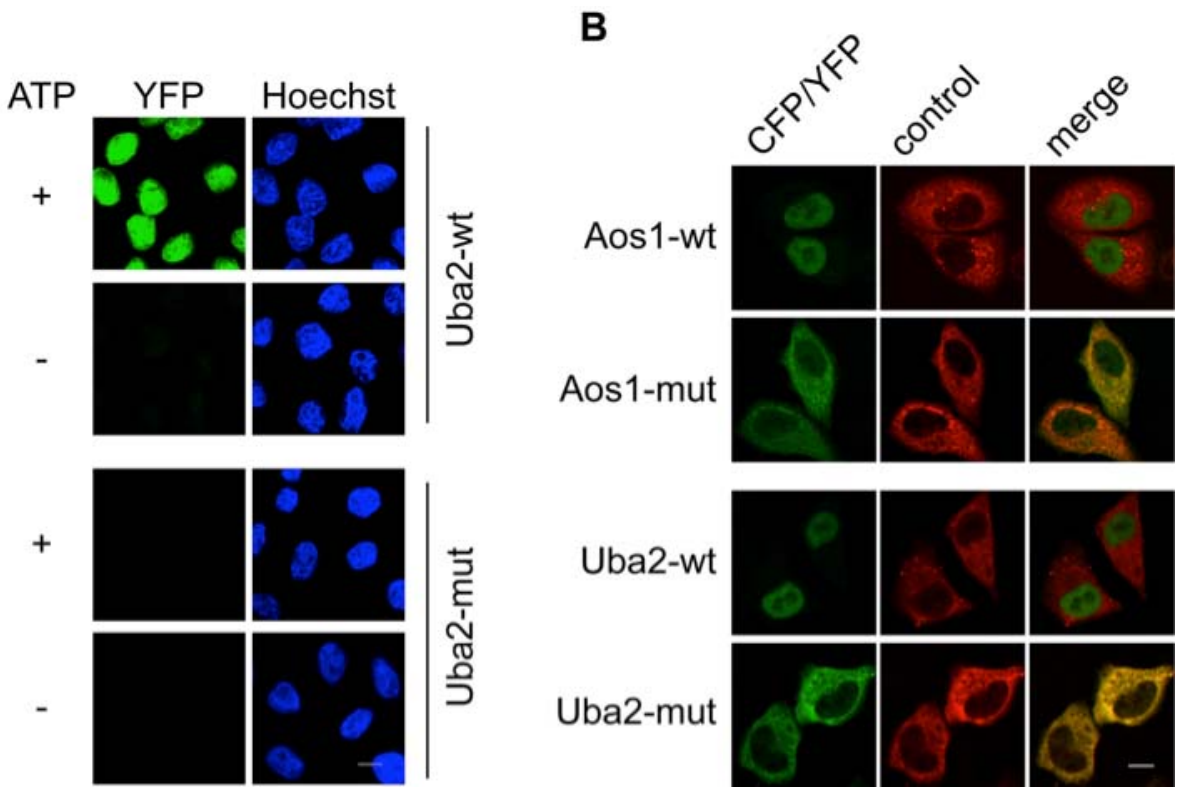

Figure 13: Aos1 and Uba2 are independently imported in HeLa cells. (A) In vitro import of recombinant purified CFP-Aos1 or Uba2-YFP by cytosolic extract in semipermeabilized HeLa cells. DNA was stained with Hoechst (blue) and nuclear accumulation of wild type proteins (CFP-Aos1-

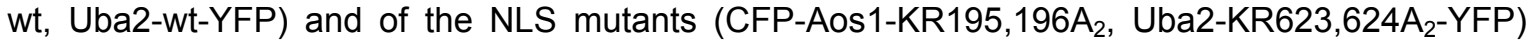
was analyzed by fluorescence microscopy (green). Assays were performed in the presence of ATP-regenerating system (ATP +), whereas negativ control experiments contained ATP-depleting system (ATP -). Bar, $10 \mu \mathrm{m}$. (B) Intracellular localization CFP-Aos1 or Uba2-YFP after microinjection into the cytoplasm of HeLa cells. Prior to injection, recombinant wildtype proteins or

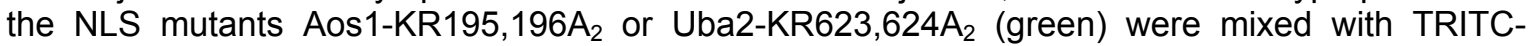
dextran which resided in the injected compartment (red). Intracellular localization was analyzed by fluorescence microscopy. Bar, $10 \mu \mathrm{m}$.

The finding that both E1 subunits contain distinct NLSs by which they can separately be imported into the nucleus could be confirmed by in vitro import assays and in vivo microinjection studies. Both recombinant wild type CFP-Aos1 and wild type Uba2YFP accumulated in the nucleus of semi-permeabilized HeLa cells in in vitro import assays with cytosolic extract (Fig. 13A) and upon microinjection into the cytoplasm of living HeLa cells (Fig. 13B). Consistent with the transfection studies, the NLS mutants CFP-Aos1-KR195,196A 2 as well as Uba2-KR623,624A $\mathrm{A}_{2}$-YFP were not imported into the nucleus (Fig. 13A, B).

\subsection{Importin $\beta$ binds via importin $\alpha$ to both Aos1 and Uba2}

The existence of independent NLSs in Aos1 and Uba2 immediately raised the question which transport receptors mediate the import of the two proteins. Since Aos1 contains a c-Myc-like import signal it may, like the c-Myc NLS peptide, interact with importin $\alpha$ and be imported via heterodimeric importin $\alpha / \beta$ (Conti et al. 2000). 
Likewise is the classical bipartite NLS of Uba2 assumed to mediate interaction with importin $\alpha / \beta$ (Dingwall et al. 1982; Robbins et al. 1991).

In order to identify transport receptors capable of binding to the SUMO E1 subunits, I performed pull-down assays with immobilized Aos1 or Uba2 on CNBr-activated sepharose testing a variety of import receptors. Figure 14A shows that heterodimeric importin $\alpha / \beta$ indeed interacted with Aos 1 wild type protein but not with the NLS mutant Aos1-KR195,196A 2 demonstrating that the interaction occurred via the identified nuclear localization signal in Aos1. Additionally, two other transport receptors, transportin and importin 13, were also capable of binding to Aos1. While binding of transportin required the NLS of Aos 1 , importin 13 bound to both wild type and NLS mutant Aos1. This result indicates that the interaction of importin 13 and

A

\section{Aos1-agarose}

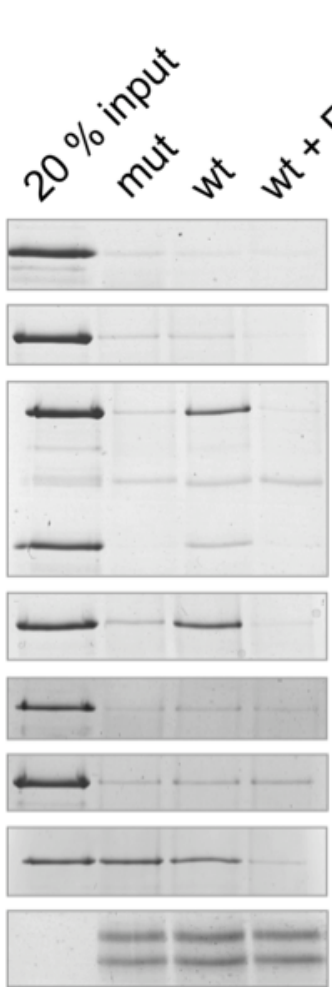

B

\section{Uba2-agarose}<smiles>[Co]=[Co]</smiles>

importin a

importin $\beta$

importin $\alpha / \beta$

transportin

importin 5

importin 7

importin 13

ovalbumin
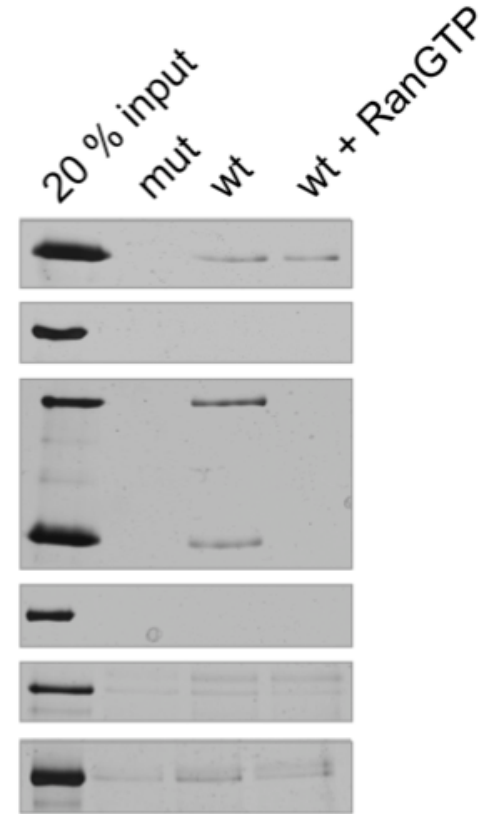

-

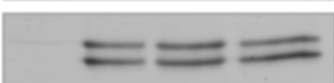

Figure 14: Importin $\beta$ binds to Aos1 and to Uba2 via the adaptor importin $\alpha$. Pulldown of recombinant import receptors importin $\alpha, \beta, \alpha / \beta$, transportin, importin 5,7 or 13 with immobilized Aos1 $(A)$ or Uba2 $(B)$. Import receptors that bound to wild type beads (Aos1-wt, Uba2-wt) and to beads with NLS mutants (Aos1-KR195,196A 2 , Uba2-KR623,624A $)$ were eluted by SDS sample buffer and compared to $20 \%$ of the input upon SDS PAGE and silverstaining. To control for specificity of binding, experiments were also performed in the presence of RanGTP that should interfere with formation of a typical receptor-cargo complex. 
Aos1 involved another region of Aos 1 than its identified NLS. However, the previous finding that the mutation of the Aos1 NLS lead to diminished nuclear import (Fig. 13, 14) suggests that importin 13, while interacting with Aos 1 in vitro, is unlikely to be the main import receptor in cells (see also below Fig. 16).

When Uba2 was immobilized only monomeric importin $\alpha$ and heterodimeric importin $\alpha / \beta$ were able to bind (Fig. 14B) demonstrating that importin $\alpha$ mediates interaction with the receptor importin $\beta$. The interactions occurred via the mapped NLS of Uba2 since the receptor heterodimer bound to wild type Uba2 but not to the immobilized NLS mutant Uba2-KR623,624A 2 (Fig. 14B).

To control specificity of the interactions detected in pull-down assays, the effect of RanGTP addition was tested. RanGTP sensitivity is characteristic for bona fide import receptor/cargo complexes. As shown in Figure 14, interactions of Aos1 with importin $\alpha / \beta$, transportin and importin 13 , and interaction of Uba2 with importin $\alpha / \beta$ are indeed RanGTP sensitive. Due to the fact that importin $\alpha$ itself is not an import receptor but only an adaptor protein, the interaction of monomeric importin $\alpha$ with Uba2 was not inhibited by RanGTP.

\subsection{Importin $\alpha / \beta$ mediates import of Aos 1 and Uba2 in vitro and in cells}

As importin $\alpha / \beta$ binds to both $\mathrm{E} 1$ subunits in a receptor/cargo-like manner, I performed in vitro import assays to test whether importin $\alpha / \beta$ can directly mediate import of Aos1 and Uba2 (Fig. 15). While importin $\alpha$ and importin $\beta$ alone were not sufficient for nuclear accumulation of CFP-Aos1 and Uba2-YFP, the heterodimeric importin $\alpha / \beta$ complex efficiently mediated nuclear import of CFP-Aos1 and Uba2YFP, but not of the NLS mutants. In control experiments, the addition of GTP-loaded RanQ69L, which binds to and blocks importin $\beta$, completely inhibited nuclear import. This indicates that the observed accumulation in the nucleus is due to active receptor-mediated nuclear import rather than passive diffusion through the nuclear pore complexes. Together, the experiments prove that the identified NLSs of Aos1 and Uba2 are indeed functional as nuclear import signals for import by dimeric importin $\alpha / \beta$.

As living cells comprise more than the import receptors tested for in vitro interaction (Fig. 14) I wanted to find out whether transport by importin $\alpha / \beta$ is the main import 


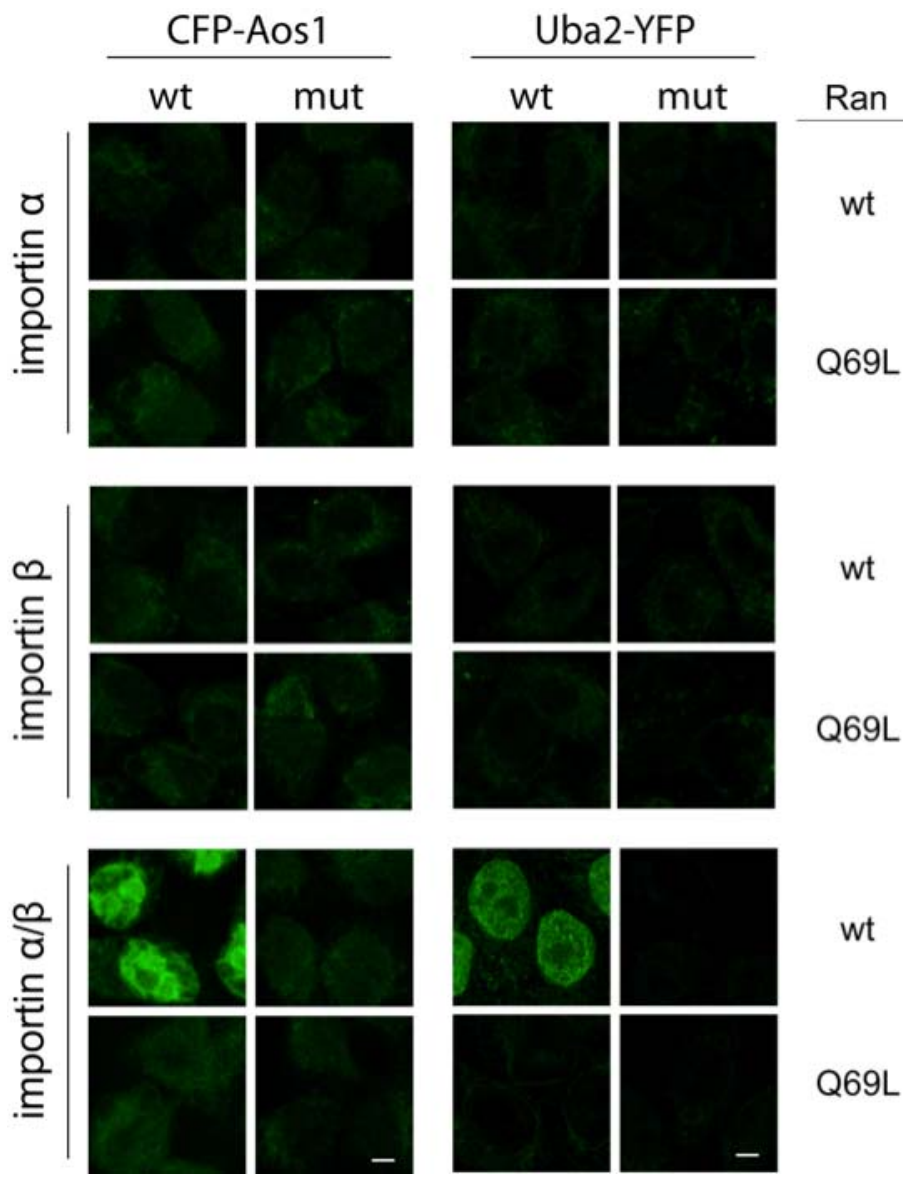

Figure 15: Importin $\alpha / \beta$ mediates in vitro import of Aos1 and Uba2. In vitro import of CFP-Aos1 or Uba2YFP by importin $\alpha, \beta$ or $\alpha$ and $\beta$ together in semipermeabilized HeLa cells. Nuclear accumulation of wild type proteins (Aos1-wt, Uba2-wt) and of the NLS mutants (Aos1KR195,196A,$\quad$ Uba2-KR623,624A ${ }_{2}$ ) was analyzed by fluorescence microscopy (green). Experiments were performed in the presence of wtRan or the mutant RanQ69L that can not hydrolyse GTP. Bar, $10 \mu \mathrm{m}$.

wt

Q69L

pathway or whether other cellular transport factors are even more potent in mediating nuclear import of Aos1 or Uba2. To address this question I microinjected fluorescent Aos1 and Uba2 into the cytoplasm of HeLa cells (Fig. 16) and compared the efficiency of nuclear import in the presence or absence of monoclonal $\alpha$-importin $\beta$ antibody, which was previously described to inhibit importin $\beta$-dependent nuclear import (Chi et al. 1995). Figure 16 shows that the import of both E1 subunits was completely abolished upon inhibition of importin $\beta$ whereas nuclear import of the control M9-NLS, which is imported by transportin (Siomi et al. 1995; Nakielny et al. 1996), remained unaffected.

In combination with the in vitro finding that importin $\beta$ can only bind to and import Aos1 or Uba2 in the presence of the adaptor importin $\alpha$ (Fig. 14, 15) these data demonstrate that nuclear import of monomeric Aos1 and Uba2 in cells is mainly mediated via the importin $\alpha / \beta$ pathway. 


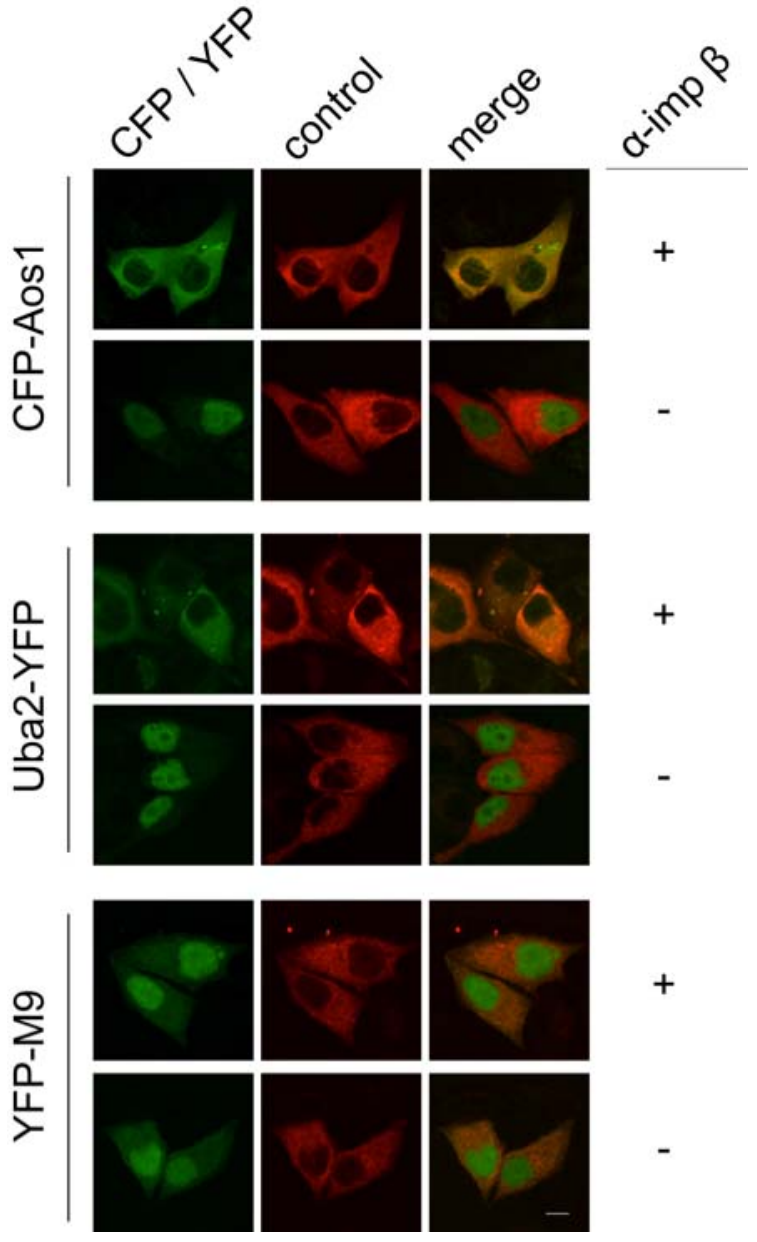

Figure 16: Importin $\beta$ mediates import of Aos1 and Uba2 in cells. Mixtures of the fluorescent target protein (green), the injection control TRITC-dextran (red) and either monoclonal inhibitory $\alpha$ importin $\beta$ antibody 3E9 $(\alpha-$ imp $\beta+)$ or transport buffer ( $\alpha$-imp $\beta \quad-)$ were microinjected into the cytoplasm of HeLa cells. Intracellular localization of CFP-Aos1, Uba2-YFP and YFP-M9 were analyzed by fluorescence microscopy. YFP-M9 is a cargo protein of the import receptor transportin (Siomi et al. 1995; Nakielny et al. 1996) and is used as control cargo whose nuclear import should be unaltered by inhibition of the importin $\beta$-transport pathway. Bar, $10 \mu \mathrm{m}$.

\subsection{Pre-assembled E1 complex can be imported into the nucleus}

While import of newly synthesized individual subunits may be sufficient to explain intranuclear localization of the SUMO E1 enzyme, preformed complexes need to reenter the nucleus after mitosis. To test whether whether assembled SUMO E1 complex can generally be imported into the nucleus, I first carried out in vitro import assays in the presence of HeLa cytosol. Figure 17A reveals that pre-formed wild type E1 complex consisting of CFP-Aos1-wt and Uba2-wt-YFP accumulated in the nuclei in an energy-dependent manner (rows 1 and 2).

This finding allowed to test nuclear import of different combinations of wild type and mutant Aos1 and Uba2. Remarkably, a complex of mutant Aos1 and wild type Uba2 (CFP-Aos1-KR195,196A 2 /Uba2-wt-YFP) also accumulated in the nuclei (rows 5 and 6). In contrast, E1 complexes with the import-defective NLS mutant of Uba2 (CFPAos1-wt/Uba2-KR623,624A ${ }_{2}$-YFP and CFP-Aos1-KR195,196A $2 / U b a 2-K R 623,624 A_{2}-$ YFP) were not imported (Fig. 17A, rows 3,4,7 and 8). Together these findings demonstrate that the NLS of Uba2, but not of Aos1, is required and sufficient for 
import of the assembled SUMO E1 complex. In addition to these in vitro findings, I investigated nuclear import of pre-assembled Aos1/Uba2 complex in vivo by microinjection in cells. As shown in Figure 17B, the complex containing wild type Uba2 localized to the nucleus (rows 1 and 3), whereas complex with Uba2-

A
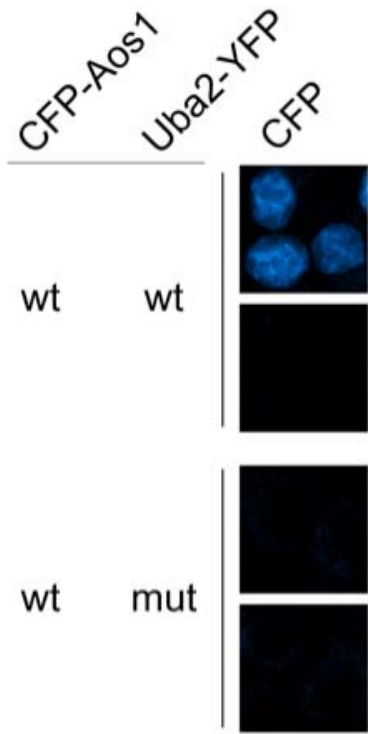

wt mut

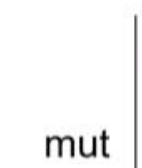

mut
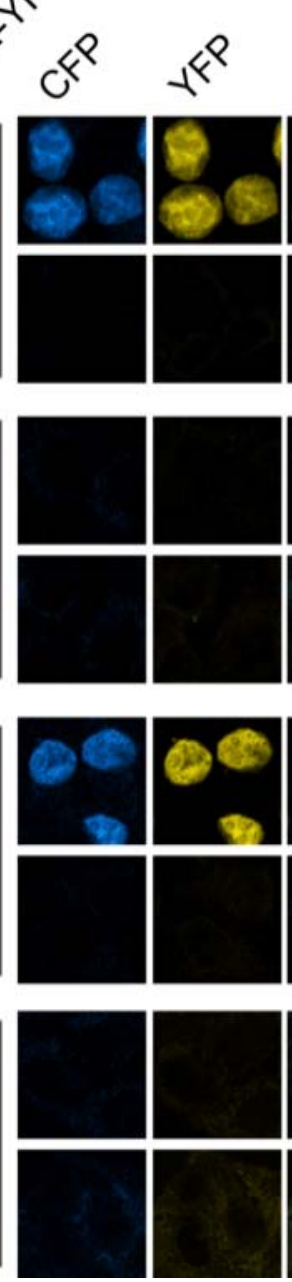

mut mut
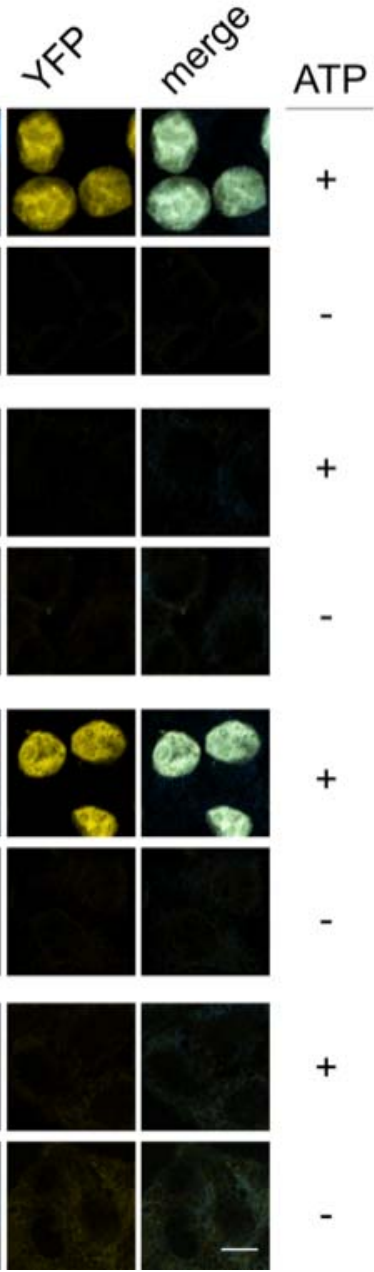

B

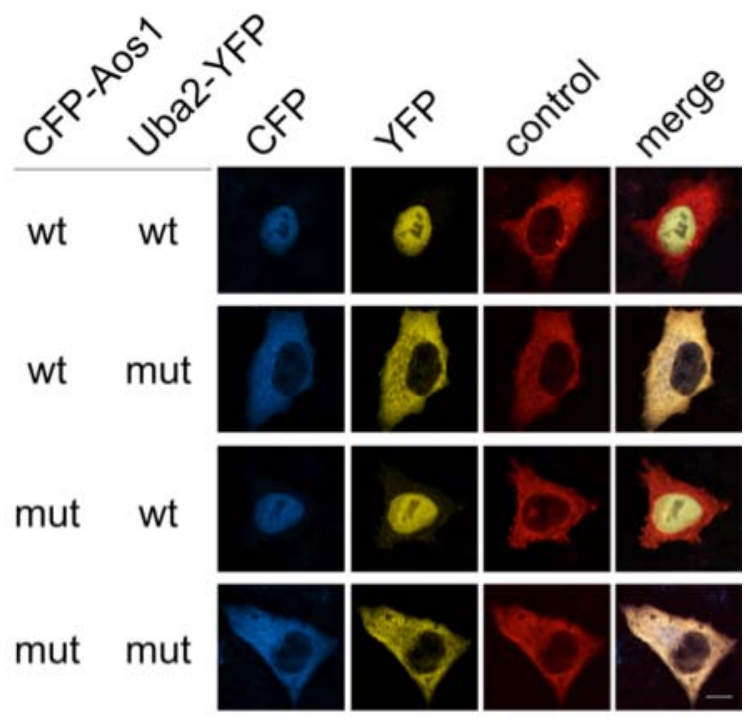

Figure 17: The Uba2 NLS is required and sufficient for import of assembled E1 complex. (A) In vitro import of different combinations of pre-assembled CFP-Aos1/Uba2-YFP complex. Prior to the experiment, complexes were reconstituted of wild type proteins (CFP-Aos1-wt, Uba2-wt-YFP) and/or NLS mutants (CFP-Aos1-KR195,196A 2 , Uba2-KR623,624A 2 -YFP). The assays were performed in semipermeabilized HeLa cells in the presence of cytosol and ATP-regenerating system (ATP +). Negativ control experiments contained ATP-depleting system (ATP -) instead. DNA was stained with Hoechst (blue) and nuclear accumulation of Aos1 (blue) and Uba2 (yellow) was analyzed by fluorescence microscopy. Bar, $10 \mu \mathrm{m}$. (B) Intracellular localization of CFP-Aos 1 and Uba2-YFP after microinjection of pre-assembled complex into the cytoplasm of HeLa cells. E1 complexes composed of wild type proteins and/or NLS mutants were reconstituted prior to the experiment as elucidated in (A). TRITC-dextran (red) is contained in the injection mix and resided in the injected compartment, thereby serving as injection control. Intracellular localization of CFPAos1 (blue) and Uba2-YFP (yellow) was analyzed by fluorescence microscopy. Bar, $10 \mu \mathrm{m}$. 
$\mathrm{KR} 623,624 \mathrm{~A}_{2}$ remained in the cytoplasm (rows 2 and 4). These microinjection experiments document that the NLS of Uba2 is indeed required and sufficient for mediating nuclear import of pre-formed SUMO E1 complex in cells.

\subsection{The NLS of Uba2 mediates E1 interaction with and import by importin $\alpha / \beta$ in vitro}

Since I identified importin $\alpha / \beta$ as the main import receptor for both $E 1$ subunits, I also analyzed its capability to interact with assembled E1 complex. For this purpose, I incubated different combinations of pre-formed E1 complex with a 5-fold molar excess of recombinant import receptors and subsequently analyzed the formation of complexes by analytical gel filtration (Fig. 18). Using a vast excess of import receptors allowed formation of receptor/cargo complexes containing more than one

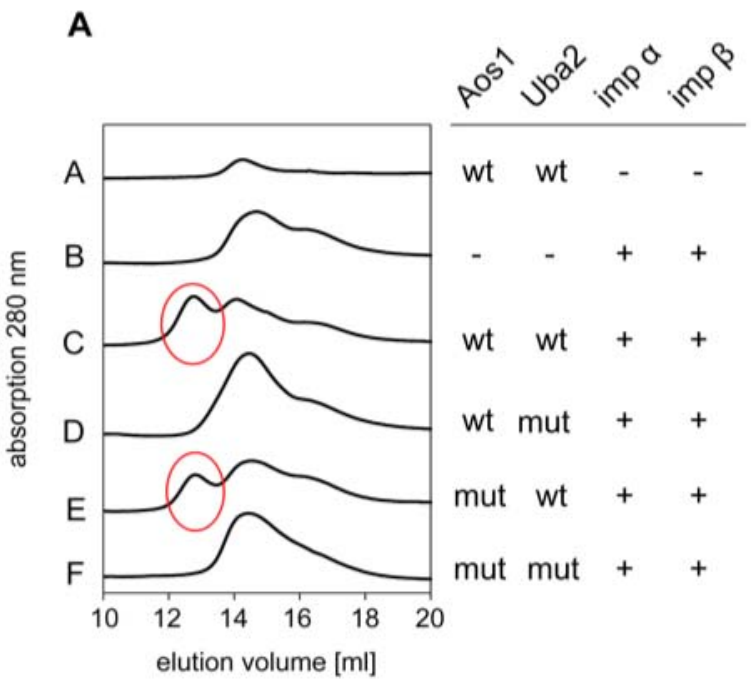

B

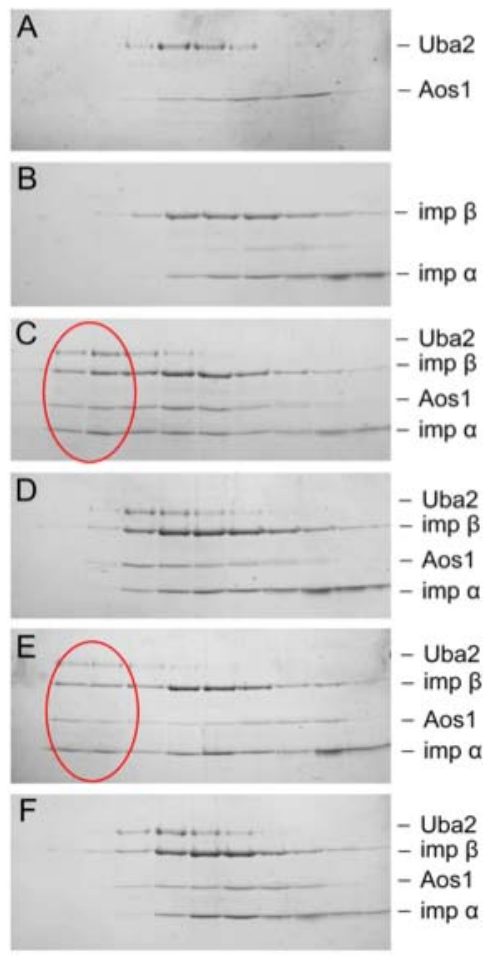

Figure 18: Importin $\alpha / \beta$ binds to assembled E1 complex exclusively via the Uba2 NLS. Analytical gelfiltration was performed after incubation of assembled CFP-Aos1/Uba2-YFP complexes with a 5 fold molar excess of importins $\alpha$ and $\beta$. E1 complexes were reconstituted of Aos1-wt (wt) or Aos1-KR195,196A (mut) and Uba2-wt (wt) or Uba2-KR623,624A 2 (mut). Elution profiles from the superose6-HR10/30 column were recorded by the Äkta purifier system (GE Healthcare) and processed with sigma plot 8.02 (Systat Software Inc.) (A). Fractions of $0.3 \mathrm{ml}$ were collected and $50 \mu \mathrm{l}$ of each fraction was analyzed by SDS PAGE and Coomassie-staining (B). $\mathrm{E} 1 /$ importin $\alpha / \beta$ complexes are highlighted in red. 
receptor per cargo complex. Compared to the running behaviour of E1 complex alone (Fig. 18A, profile 1) or importins alone (profile 2), the elution-profile of Aos1wt/Uba2-wt pre-incubated with importin $\left\langle/{ }^{\circledR}\right.$ showed an additional high-molecular weight peak eluting from the column at approximately $12.5 \mathrm{ml}$ (profile 3). Subsequent SDS PAGE analysis revealed that this peak contained a complex composed of Aos1/Uba2/importin $\alpha / \beta$ in equimolar ratios (Fig. 18B, gel 3). A peak at the same elution volume was also detected upon incubation of importin $\alpha / \beta$ with Aos 1 mut/Uba2-wt. This complex showed no difference compared to the wild type complex (profile and gel 5). In contrast, no complex formation was detected when the NLS in Uba2 was mutated (profiles and gels 4 and 6), demonstrating that the Uba2 NLS is absolutely required for formation of the tetrameric import complex Aos $1 /$ Uba2/importin $\alpha / \beta$.

Next, I wanted to test whether the tetrameric Aos $1 /$ Uba2/importin $\alpha / \beta$ complex is functional in nuclear transport. Therefore, I tested the ability of recombinant importin $\alpha / \beta$ to mediate nuclear import of different pre-assembled $E 1$ complexes in in vitro

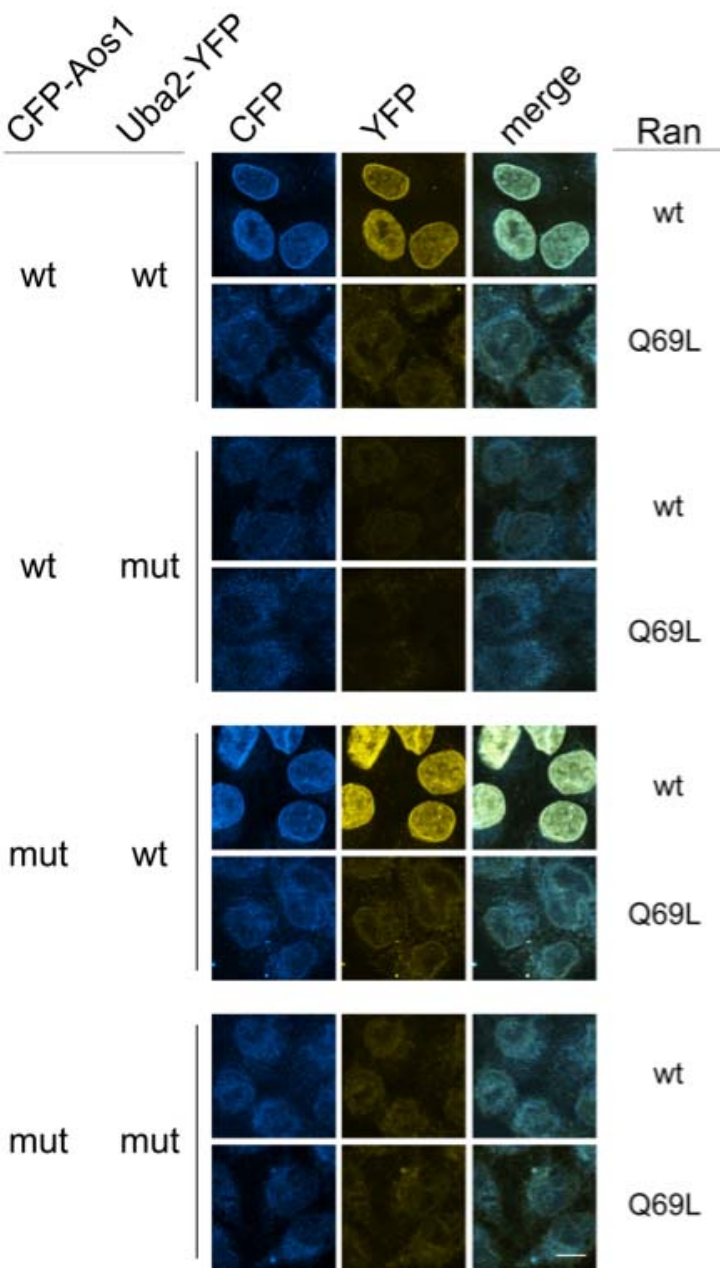

Figure 19: In vitro nuclear import of Aos1/Uba2 complex is mediated by importin $\alpha / \beta$ via interaction with the Uba2 NLS. In vitro import of preassembled CFP-Aos1/Uba2-YFP complexes by importin $\alpha, \beta$ or $\alpha$ and $\beta$ together. E1 complexes are composed of CFP-Aos1-wt (wt) or CFP-Aos1KR195,196A ${ }_{2}$ (mut) and Uba2-wt-YFP (wt) or Uba2-KR623,624A - YFP (mut). The experiments were performed in the presence of wt Ran or RanQ69L. Nuclear accumulation of CFP-Aos1 (blue) and Uba2-YFP (yellow) was analyzed by fluorescence microscopy. Bar, $10 \mu \mathrm{m}$. 
import assays (Fig. 19). SUMO E1 complex containing wild type Uba2 (rows 1, 2, 5 and 6) strongly accumulated in the nuclei in the presence of wild type Ran, whereas E1 complex with the NLS mutant Uba2-KR623,624A 2 -YFP was not imported (rows 3 , 4, 7 and 8). Consistent with the microinjection data, application of wild type or NLS mutant Aos1 did not influence the import behaviour of the pre-formed E1 complex (comparison of the rows 1 and 5 or 3 and 7). As expected, RanQ69L inhibited nuclear import.

Altogether, these results demonstrate that the NLS of Uba2 is required and sufficient for the interaction of pre-assembled E1 with importin $\alpha / \beta$ and resulting nuclear import of E1 complex.

\subsection{The SUMO E1 complex in cells is mainly imported by importin $\alpha / \beta$}

The finding that the NLS of Uba2 is required for E1 import upon microinjection into living cells (Fig. 17B) in combination with the fact that the heterodimer importin $\alpha / \beta$ is required to import Uba2 alone (Fig. 14B, 15, 16) leads to the suggestion that the importin $\alpha / \beta$-pathway is also the main cellular import pathway for assembled E1.

To test whether any cellular import receptor other than importin $\beta$ is capable of mediating nuclear import of E1 complex, I analyzed the effect of inhibitory $\alpha$-importin $\beta$ antibody on import of micorinjected E1 comeplex (Fig. 20). Indeed, inhibition of importin $\beta$ completely abolished nuclear import of E1 complex, which allows to conclude that the importin $\alpha / \beta$-pathway is the main import-pathway for assembled

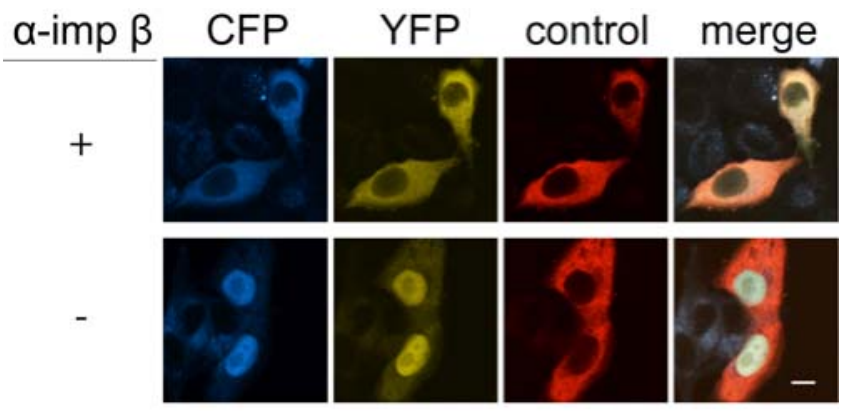

Figure 20: Inhibition of importin $\beta$ prevents nuclear import of assembled E1 complex in cells. Mixtures of CFP-Aos1/Uba2-YFP complex (blue and yellow), the injection control TRITCdextran (red) and either inhibitory $\alpha$-importin $\beta$ antibody $(\alpha$-imp $\beta+)$ or transport buffer $(\alpha$-imp $\beta-)$ were microinjected into the cytoplasm of HeLa cells. Intracellular localization of CFP-Aos1 (blue) and Uba2-YFP (vellow) were analyzed by confocal fluorescence microscopy. Bar, $10 \mu \mathrm{m}$. 
Aos1/Uba2 complex in HeLa cells.

In conclusion, the results described here reveal that nuclear E1 complex can be generated in two ways (Fig. 21). Single subunits can be imported by importin $\alpha / \beta$ independently of each other via distinct NLSs mapped in Aos1 and Uba2 (left side); in addition, the pre-assembled Aos1/Uba2 complex can undergo nuclear import by importin $\alpha / \beta$ via interaction with the NLS in Uba2 (right side).

\section{Analysis of the cytoplasmic E1 pool}

Published work on the localization of the two SUMO E1 subunits Aos1 and Uba2

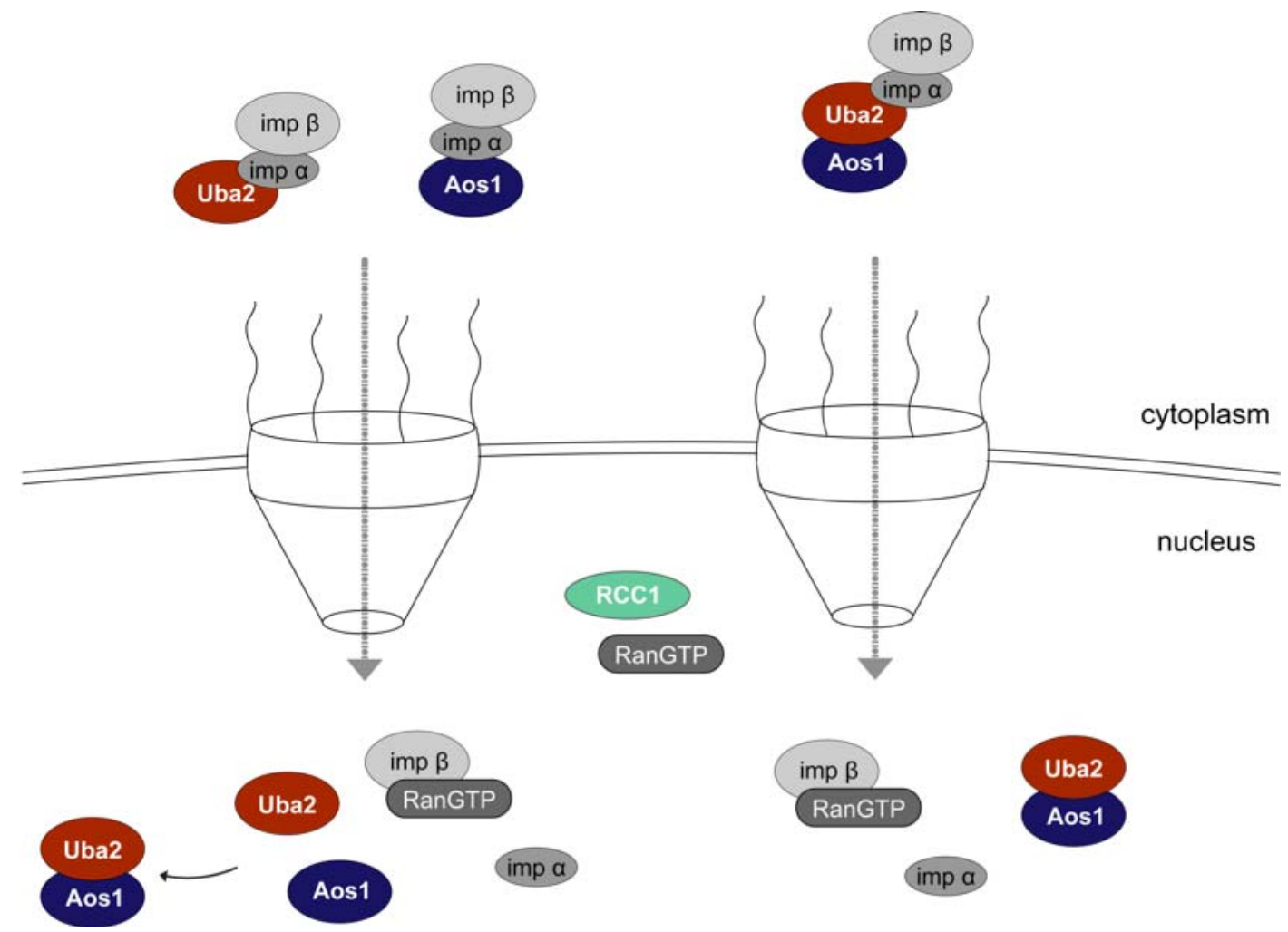

Figure 21: Schematic model of the generation of active nuclear E1 complex. (left) The single subunits Aos1 (blue) and Uba2 (red) are idependently imported by importin $\alpha / \beta$ via their distinct NLSs. After translocation through the nuclear pore the cargoes are released into the nucleus and can assemble to active SUMO E1 complex. (right) Already formed E1 complex interacts via the NLS of Uba2 with importin $\alpha / \beta$, which mediate the translocation into the nucleus.

revealed that both proteins predominantly localize in the nuclear compartment (Dohmen et al. 1995; Azuma et al. 2001; Pichler et al. 2002). However, data from Dr. 
Guillaume Bossis and Dr. Andrea Pichler showed the presence of both subunits also in cytosolic fractions and even pointed to a regulatory event specific to the cytosolic E1 pool (Pichler et al. 2002; Bossis et al. 2006). Furthermore, preliminary results obtained by Dr. K. Chmielarska in our lab suggested an enrichment of Aos1 at the Golgi apparatus (K. Chmielarska, PhD thesis). To obtain a more coherent picture of E1's localization I aimed to perform a more detailed analysis of the intracellular localization of the SUMO E1 enzyme in HeLa cells and hippocampal neurons.

\subsection{Endogenous Aos1/Uba2 predominantly localizes to the nucleus of}

HeLa cells

Previous immunofluorescence studies using epifluorescence microscopes suggested that the main pool of SUMO E1 resides in the nuclei of cells (Dohmen et al. 1995; Azuma et al. 2001; Pichler et al. 2002). As epifluorescent microscopes detect the emitted light from the whole, or at least multiple layers of the specimen, the resulting image displays the sum of fluorescent intensities of all these layers. As the nucleus of a cell is thicker than the cytoplasm, a picture from a signal equally distributed between these compartments might therefore show increased signal intensity in the nucleus compared to the cytoplasm. Consequently, the epifluorescent images from the literature, which illustrated the nuclear enrichment of SUMO E1 in adherent cells, might lead to a partially wrong conclusion regarding the enzymes's distribution

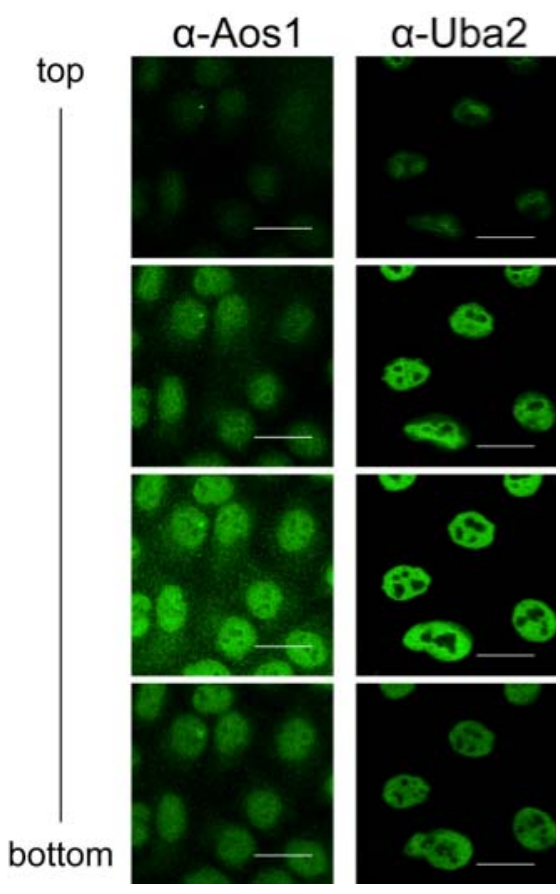

Figure 22: Endogenous Aos1 and Uba2 predominantly localize to the nucleus. Fixed HeLa cells were immunostained with affinity purified goat $\alpha$ Aos 1 or goat $\alpha$-Uba2 antibody/donkey $\alpha$-goat-Alexa488 (green). Images from different confocal planes through the cells were acquired by confocal laser scanning microscopy using a confocal LSM510meta (Zeiss). Bar, $20 \mu \mathrm{m}$. 
between nucleus and cytoplasm. In order to analyze the intracellular distribution of endogenous Aos1 and Uba2 more accurately I verified the subcellular localization of the proteins by indirect immunofluorescence analyzed with confocal microscopy. Using this technique, only fluorescent light emitted very close to the focal plane in the specimen was detected, producing images with signal intensities that better reflect the intracellular concentrations of the fluorophores.

Images from different confocal planes ranging from the top to the bottom of the cells (Fig. 22) illustrate that endogenous SUMO E1 was significantly higher concentrated in the nucleus than in the cytoplasm. Aos1, but not Uba2, was to some extent also detected in the cytoplasm. This finding might be explained by an access of Aos1 over Uba2, which has previously been shown to occur during cell cycle progression (Azuma et al. 2001). A reason why endogenous Uba2 was not detected in the cytoplasm could be that high affinity epitopes of the protein are masked in the cytoplasm or that the amount of cytoplamic Uba2 is simply below the detection limit of the antibody. Consequently, this result confirms a predominant nuclear localization of both SUMO E1 subunits obtained by previous studies with epifluorescent microscopic analysis (Dohmen et al. 1995; Azuma et al. 2001; Pichler et al. 2002).

\subsection{SUMO E1 is found in cytosolic fractions of HeLa cells}

To address the question of the intracellular localization with an additinoal method that allows to detect small pools of Aos1/Uba2, I performed differential centrifugation of HeLa cells to obtain fractions of different cellular compartments. Equal protein amounts of each fraction were analyzed by SDS PAGE and immunoblotting to verify the presence of specific proteins (Fig. 23). Detection of the marker proteins RCC1 (nucleus), LDH (cytosol) and GM130 (Golgi apparatus) in the predicted fractions illustrated the successful separation of the different cellular compartments. In line with previous data from Dr. Guillaume Bossis and Dr. Andrea Pichler both SUMO E1 subunits Aos1 and Uba2 were clearly present in the nuclear and cytosolic fractions (Fig. 11, lane 1, 2, SN) (Pichler et al. 2002; Bossis et al. 2006). Interestingly, in contrast to the cytosolic marker protein LDH, Aos1 was also found in fraction 3 and Uba2 was found in fractions $3-6$, demonstrating that Aos1 and Uba2 are also present in other compartments besides nucleus and cytosol. The detection of both subunits in fraction 3, which contains proteins of the Golgi apparatus (apparent from the Golgi marker GM130), is in line with preliminary data from Dr. Katarzyna 


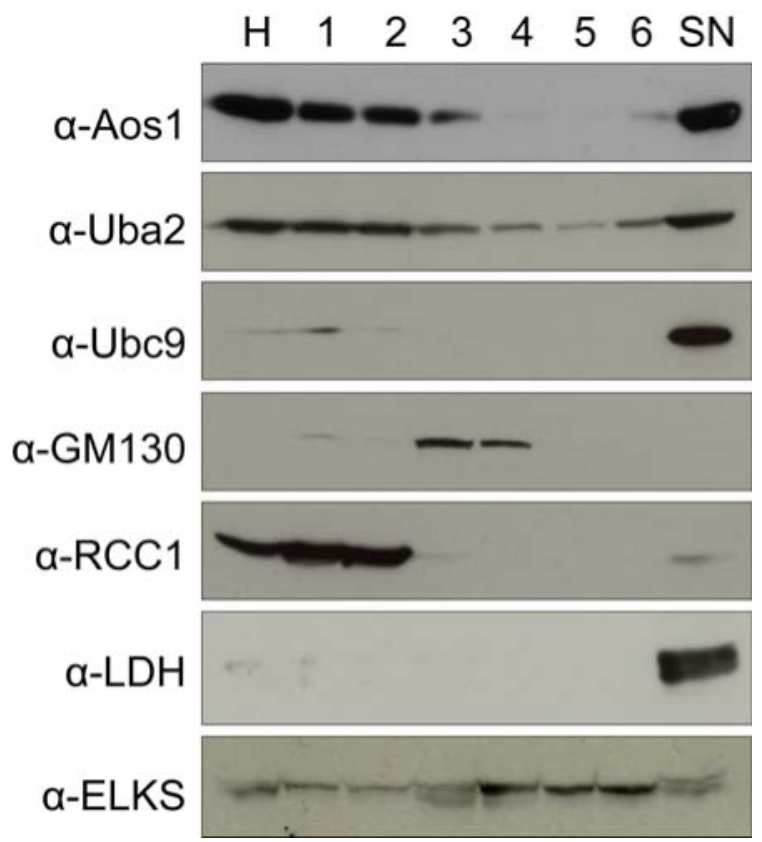

Figure 23: Aos1 and Uba2 are present in the cytososlic fraction of HeLa cells. Fractions of lysed HeLa suspension cells were obtained by differential centrifugation. Protein equivalents of the homogenate $(H)$, fractions containing whole cells and intact nuclei (1), nuclear debris (2), mitochondria, lysosomes and golgi (3), lysosomes, golgi and peroxisomes (4), endoplasmic reticulum (5), polysomes (6) and of the cytosolic fraction (SN) were analyzed by immunoblotting with $\alpha$-Aos1, $\alpha$-Uba2, $\alpha$ Ubc9, $\alpha$-GM130, $\alpha$-RCC1, $\alpha$-LDH and $\alpha-$ ELKS for the presence of these proteins.

Chmielarska indicating the localization of Aos1 and Uba2 at the Golgi apparatus (K. Chmielarska, PhD thesis).

While analysis of protein equivalents, shown in Figure 23, reveals the presence of E2 enzyme mainly in the cytosol but not in the homogenate, analysis of cell equivalents show that the SUMO E2 is also present in the nuclear fraction as well as in the homogenate (data not shown). The analysis of cell equivalents (not shown) reveals that the amounts of Aos1, Uba2, and Ubc9 in the nuclear fraction was lower than the amounts detected in the cytosolic supernatant. This finding coincides with previous fractionation experiments performed by Dr. Andrea Pichler (Pichler et al. 2002) and contrasts the result of immunofluorescence analysis (Fig. 22), which revealed significantly higher amounts in the nucleus. A possible explanation for the observed discrepancy may be the fractionation procedure: The incubation on ice or the hypotonic swelling may trigger active export into the cytosol, or the lysis procedure slightly damages the nuclei and thereby causes leaking out of the nuclei.

Taken together, the immunofluorescence analysis of the distribution of Aos 1 and Uba2 illustrates that the endogenous E1 subunits are predominantely localized in the nucleus of cells. While a pool of Aos1 was also found in the cytoplasm, Uba2 was only found in the nucleus. However, fractionation experiments verify the presence of both $\mathrm{E} 1$ subunits in the cytosol of cells. Consequently, the question how much SUMO E1 enzyme is located in the cytoplasm remains open. 


\subsection{Distribution of E1 in neuronal cells is analogous to HeLa cells}

To expand the analysis of the intracellular localization of Aos1/Uba2, I turned to neuronal cells, where SUMOylation of synapse proteins like the transmembrane kainate-receptor subunit GluR6 and the metatropic glutamate receptor mGluR8 has been found to play a role in the regulation of synaptic function (Tang et al. 2005; Martin et al. 2007; reviewed in Coussen et al. 2007; Martin et al. 2007). These previous data raise the question whether the intracellular distiribution of the SUMO E1 enzyme in neuronal cells differs from the localization in HeLa cells.

To address this question, I analyzed the intracellular distribution of Aos1 and Uba2 in subcellular fractions of hippocampal neurons from mice (Fig. 24). Samples of the fractionation of isolated cells were kindly provided by the laboratory of Prof. Dr. Nils Brose (Max-Planck Institute for Experimental Medicine, Göttingen). Enrichment of the marker protein NMDAR1 comparing the homogenate $(\mathrm{H})$ and the fraction of synaptic plasma membranes (SPM) served as an established indicator to control for successful SPM purification. Aos1 and Uba2 were detected in soluble fractions, mainly in the supernatant after nuclear sedimentation (S1) and in the cytosolic fraction (S3). Significantly, both subunits were also found in the cytosolic

A

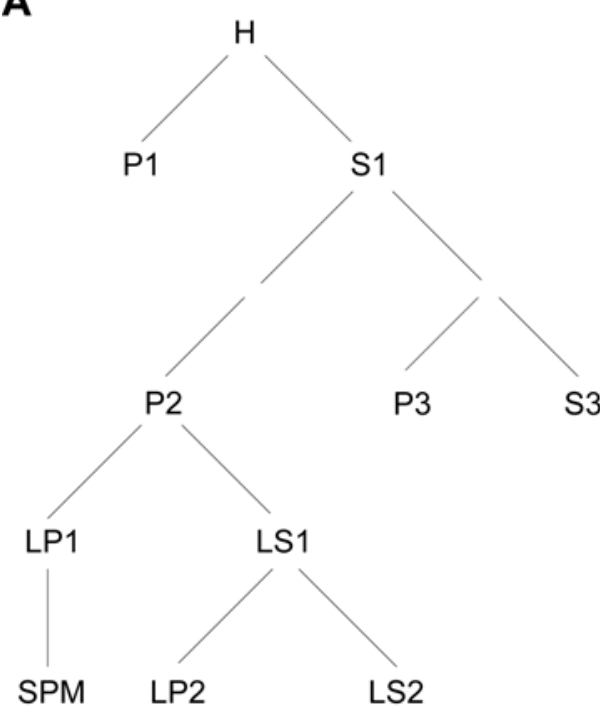

B

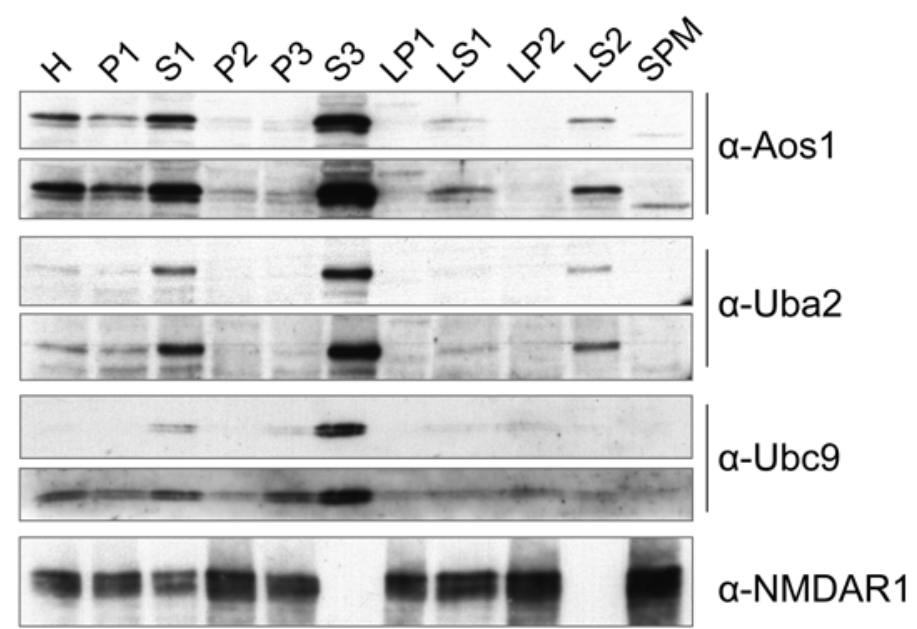

Figure 24: Aos1 and Uba2 are present in the cytosolic synaptosomal fraction of murine hippocampal neurons. Samples of fractionated hippocampal neurons from mice were kindly provided by the lab of Prof. Dr. Nils Brose (Max-Planck Institute for Experimental Medicine, Göttingen). (A) Schematic outline of the fractionation procedure according to (Jones et al. 1974). (B) Protein equivalents of the homogenate $(\mathrm{H})$, nuclear pellet (P1), supernatant after nuclear sedimentation (S1), crude synaptosomal pellet (P2), light membrane pellet (P3), cytosolic fraction (S3), heavy membrane pellet (LP1), intermediate fraction LS1 (LS1), crude synaptic vesicles (LP2), cytosolic synaptosomal fraction (LS2) and synaptic plasma membranes (SPM) were analyzed by immunoblotting with $\alpha$-Aos1, $\alpha$-Uba2, $\alpha$-Ubc9, $\alpha$-NMDAR1. 
synaptosomal fraction (LS2), revealing that some E1 indeed localizes to the cytoplasm of neuronal synaptic cells. The fact that the detected signal of Aos1 and Uba2 in the LS2 fraction was relatively low compared to S3 is not problematic, since the fractions were differentially purified from the fraction $\mathrm{S} 1$ and can therefore not be directly compared (Fig. 12A). However, the purification of LS2 from the crude synaptosomal pellet (P2) over LS1 goes along with a clear enrichment of Aos1 and Uba2, indicating the significance of the detected signal. For Ubc9 a similar enrichment in S1 and S3 was observed, but the protein was not found in fractions derived from the crude synaptosomal pellet P2.

To gain further insights into the intracellular localization of the SUMO E1 in different neuronal cells, I performed immunohistochemical analyses of endogenous Uba2 in tissue slides from normal mouse brain (Fig. 25). For this purpose commercially available tissue slides were deparaffinized, rehydrated and stained using $\alpha$-Uba2 antibody and Hoechst. The resulting images presented in Figure 13 exemplary show DNA, Uba2 and background fluorescence from four different regions of the brain. The samples displayed strong autofluorescence in the green and in the red channel, whereby the signal from the Uba2-staining was overlaid with background fluorescence. Comparing the background signal of the red channel with the green channel, the images from the regions 1 and 2 did not show any Uba2 signal above fluorescent background, whereas in the regions 3 and 4 specific Uba2 signal was detected. This Uba2-specific signal co-localized with Hoechst-stained DNA, in line with the predominantly nuclear Uba2 localization in cultured fibroblasts. However, no conclusions about the absence or presence of a smaller cytoplasmic pool of Uba2 could be drawn due to the high background fluorescence. Consequently, this experiment does not allow further analysis of the distribution of SUMO E1 in neuronal cells. Whether the finding that Uba2 was not detectable at all in some regions suggests cell type-specific expression of Uba2 or wheter it was due to technical problems requires further experiments. 


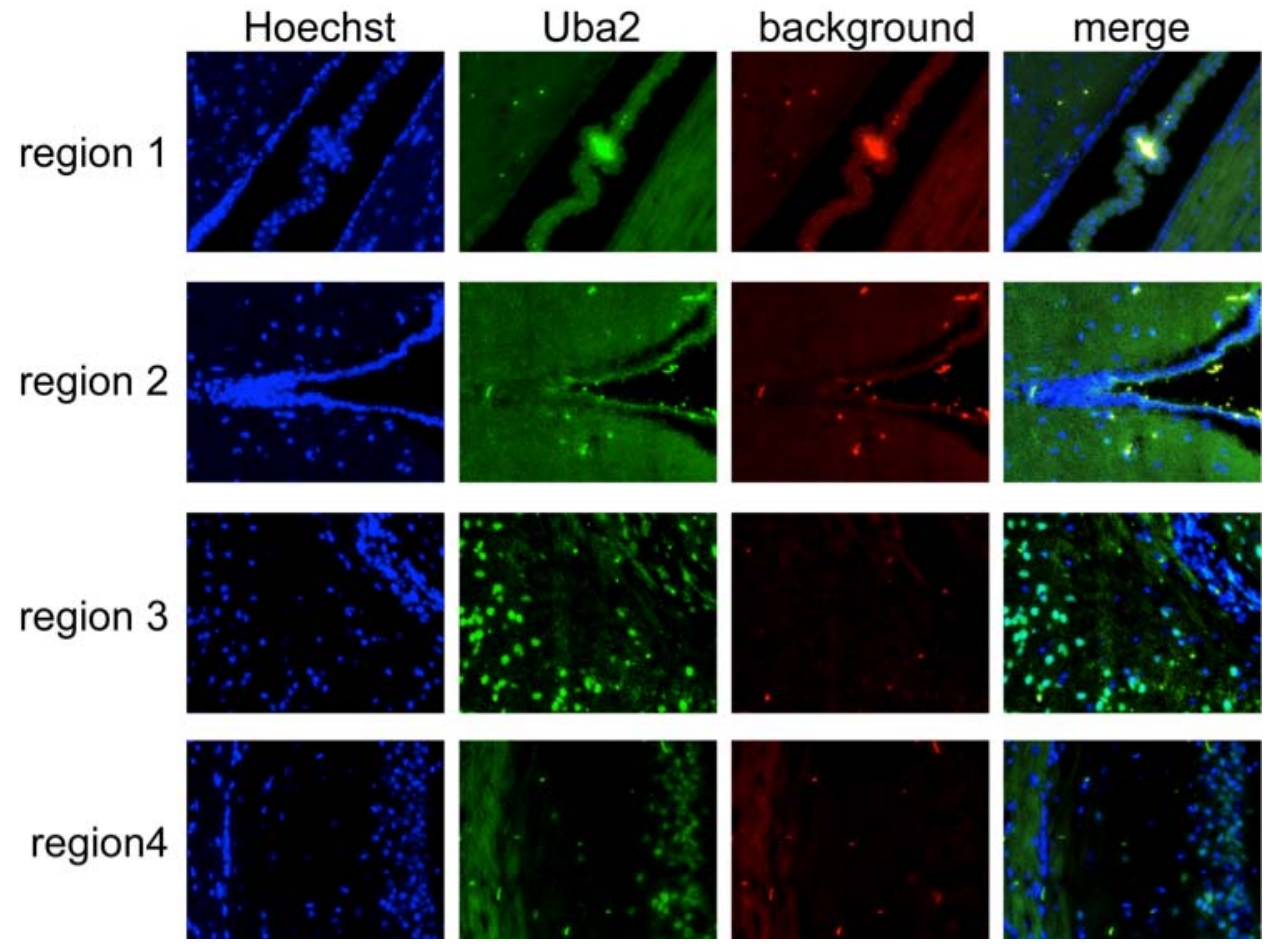

Figure 25: Detection of endogenous Uba2 in slides of murine brain. Tissue slides from murine brain were immunohistochemically stained with goat $\alpha$-Uba2/donkey $\alpha$-goat-Alexa488 (green), DNA was stained with Hoechst (blue) and autofluorescence background of the samples was detected in the red chanel (red). Images were acquired by fluorescence microscopy using a CellObserver (Zeiss).

The results obtained by analysis of neuronal cells reveal the presence of $E 1$ in cytosolic synaptosomal fractions purified from murine brain but point to predominantly nuclear localization of the enzyme, as described for HeLa cells. As already mentioned, a number of identified SUMO targets are transmembrane proteins localized and modified at the synapses of neuronal cells (Tang et al. 2005; Martin et al. 2007; reviewed in Coussen et al. 2007; Martin et al. 2007). Whether SUMOylation of these targets requires the localization of a small E1 pool at the synapses and whether this localization of the E1 is due to passive diffusion or to an active transport mechanism are very interesting questions for future studies.

\subsection{No indication for active export of the SUMO E1}

The existence of a small cytoplasmic pool of Aos1/Uba2 immediately raises the question how this pool is generated. As both E1 subunits are predominantly localized in the nucleus, active shuttling between the nucleus and the cytoplasm could be one mechanism underlying the generation of the small cytoplasmic E1 pool. For this 
reason I tested whether the E1 enzyme can be actively exported from the nucleus into the cytoplasm.

\section{Aos1 and Uba2 do not interact with CRM1 in vitro}

CRM1 is the major receptor for the export of proteins out of the nucleus (Fornerod et al. 1997; Stade et al. 1997; reviewed in Hutten et al. 2007). In most instances, the export complex is formed via interaction of CRM1 with a leucine-rich nuclear export signal (NES) within cargo proteins in the presence of RanGTP (Fischer et al. 1995). The fact that most proteins are exported via CRM1 in combination with the finding that potential NES consensus sequences $L-X_{2-3}(L, I, V, M, F)-X_{2-3}-L-X-(L, I, V)$ are present in Aos1 and in Uba2 (Fig. 26A) make CRM1 a promising candidate for mediating potential nuclear export of the SUMO E1. However, analysis of the Aos1/Uba2 structure revealed, that the consensus NESs are located at the interaction surfaces of both subunits, for which reason interaction CRM1 with assembled E1 complex is rather unlikely whereas the NESs are accessible in the monomeric subunits (Fig. 26B).

To test whether Aos1, Uba2 or assembled E1 complex binds to the export receptor CRM1 I performed pull-down experiments with immobilized CRM1. However,

A

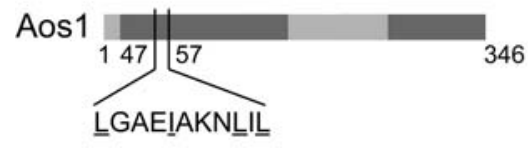

Uba2

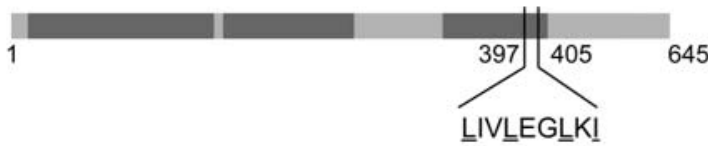

B

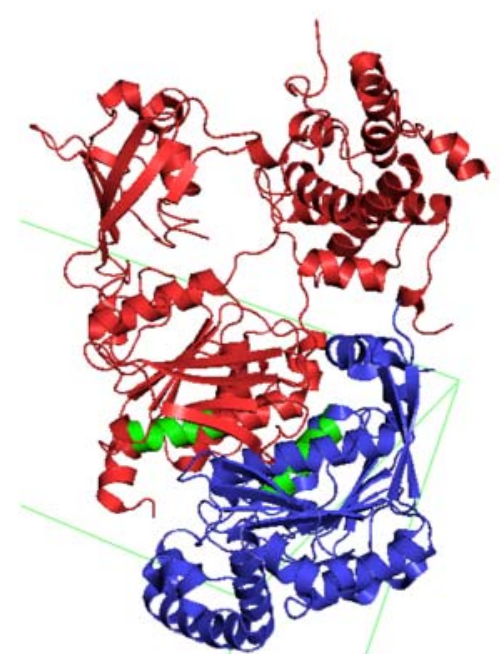

Figure 26: Aos1 and Uba2 contain a potential consensus NES. (A) Schematic illustration of the position and the sequence of the potential consensus NES in Aos1 and Uba2. (B) Illustration of the spatial localization of the potential NESs of Aos1 and Uba2 in a cartoon of the crystal structure of heterodimeric Aos1/Uba2 complex solved by Lois et al. (Lois et al. 2005). Aos1 is coloured in blue, Uba2 is shown in red and the potential consensus NESs are highlighted in green. The raw data were obtained from the Protein Data Bank (PDB) and images were generated with PyMOL v0.99 (DeLano Scientific LLC). 


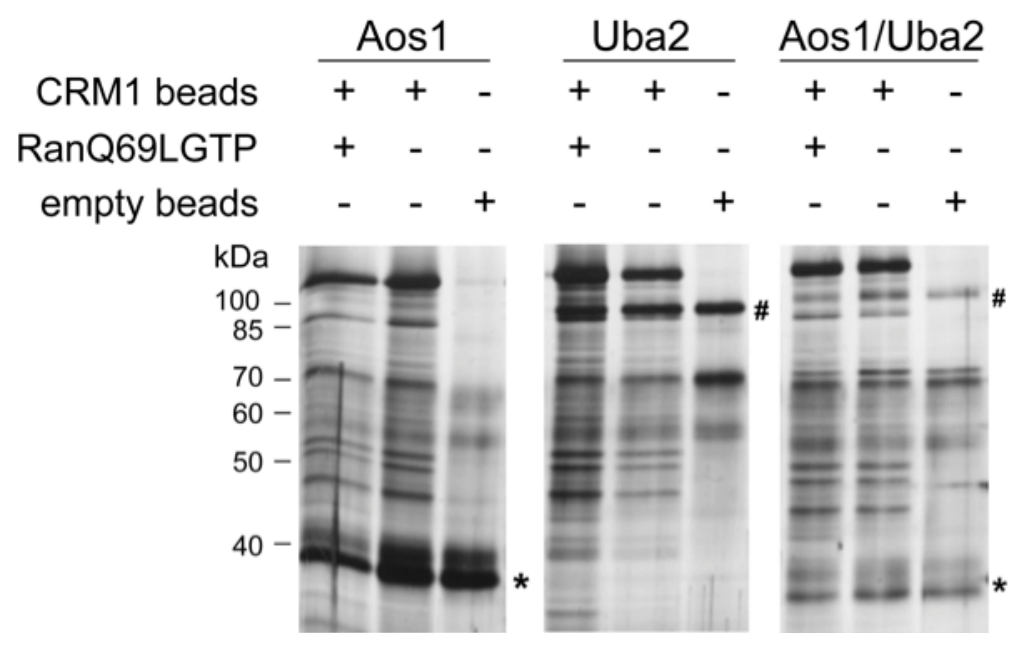

Figure 27: No interaction of Aos1 or Uba2 with immobilized CRM1 in pulldown assays. Aos1 $\left({ }^{*}\right)$, Uba2 $\left(^{\#}\right)$ or assembled E1 complex $\left({ }^{*},{ }^{*}\right)$ were subjected to CRM1 binding by incubation with beads containing immobilized CRM1. The assays were performed in the presence of RanQ69L whereas the negative control experiments did not contain Ran. Unspecific binding to beads was verified with empty beads. Bead bound proteins were analyzed by SDS PAGE and visualized by silver-staining.

comparable amounts of Aos1, Uba2 and assembled E1 comples bound to CRM1 beads and to empty beads (Fig. 27). This strong background binding to the empty beads indicates that the conditions of the pull-down experiments were not appropriate for the analysis of any specific interaction of the potential cargoes with CRM1.

Therefore, I turned to RanGAP assays, which are well-established experiments for analysis of CRM1-cargo interactions. The assay is based on the fact that RanGTP is protected from RanGAP-stimulated GTP-hydrolysis when RanGTP is present in a trimeric CRM1/RanGTP/cargo export complex, while in the absence of cargo RanGTP is hydrolyzed as assessed by the production of free phosphate. This method is applicable to verify even weak or transient interactions between CRM1 and NES bearing cargo proteins (Paraskeva et al. 1999; Engelsma et al. 2004). The positive control experiment in the presence of the NES peptide (NS2 protein of minute virus of mice, CVDEMTKKFGTLTIHDTEK; Askjaer et al. 1999) caused a significant decrease of GTP-hydrolysis by RanGAP (Fig. 28, reactions 4 and 5). The amount of free $\gamma$-phosphate decreased to $40 \%$ compared to the reactions without any export signal containing cargo (reactions 2, 3 and 5), indicating that the NES peptide allowed the assembly of export complexes and thereby protected RanGTP from hydrolysis. In contrast, GTP-hydrolysis was unaltered upon addition of Aos1, 


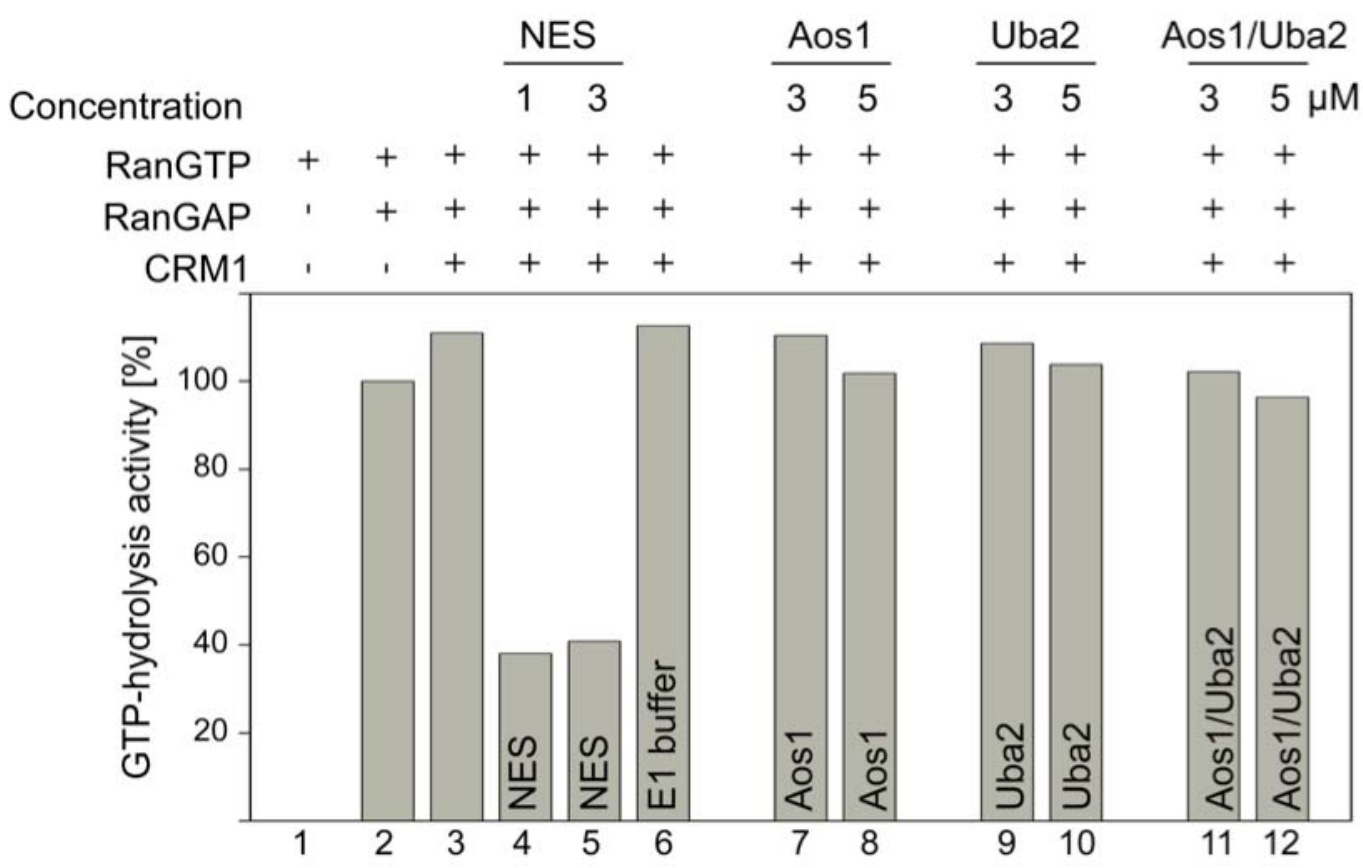

Figure 28: Aos1, Uba2 or assembled E1 do not interact with CRM1 in RanGAP assays. Aos1, Uba2 or assembled Aos1/Uba2 complex (3 or $5 \mu \mathrm{M}$ final) were incubated with CRM1 (1 $\mu \mathrm{M})$, RanGAP (60 nM), GTP $(200 \mathrm{nM})$ and a limiting amount of $\left[\gamma^{32} \mathrm{P}\right] \operatorname{RanGTP}(30 \mathrm{nM})$. The reaction was stopped, $\left[\gamma-{ }^{32} \mathrm{P}\right]$ RanGTP was removed by centrifugation with charcoal and the released $\left[\gamma_{-}{ }^{32} \mathrm{P}\right]$ was measured by scintillation counting. Background counts from a reaction without RanGAP were subtracted and GTP hydrolysis was expressed as the percentage of the value of radioactivity measured in a reaction with RanGAP and without CRM1. Positive control reactions contained nuclear export signal (NES) $(1$ or $3 \mu \mathrm{M})$ instead of SUMO E1.

Uba2 or assembled E1 complex (reactions 7 to 12), revealing that neither the single E1 subunits nor pre-formed E1 complex interact with the export receptor CRM1.

While CRM1 is the best-characterized export receptor, it certainly is not the only one. Other export receptors, as well as the transport receptors transportin and importin 13, primarily known for their import activity, have been shown to also mediate nuclear export of selected cargoes (Gallouzi et al. 2001; Mingot et al. 2001; Shamsher et al. 2002). Interestingly, transportin and importin 13 have both been shown to interact with Aos1 in in vitro pull-down assays, raising the question whether one of these transport receptors can mediate export of SUMO E1. However, their interaction with SUMO E1 subunits was inhibited in the presence of RanGTP (see Fig. 18) necessitating a more complex mechanism.

No evidence for shuttling of CFP-Aos1 in HeLa cells

To test in cells whether SUMO E1 is in general actively exported from the nucleus I analyzed the shuttling behaviour of Aos 1 in interspecies heterokaryon assays. For 


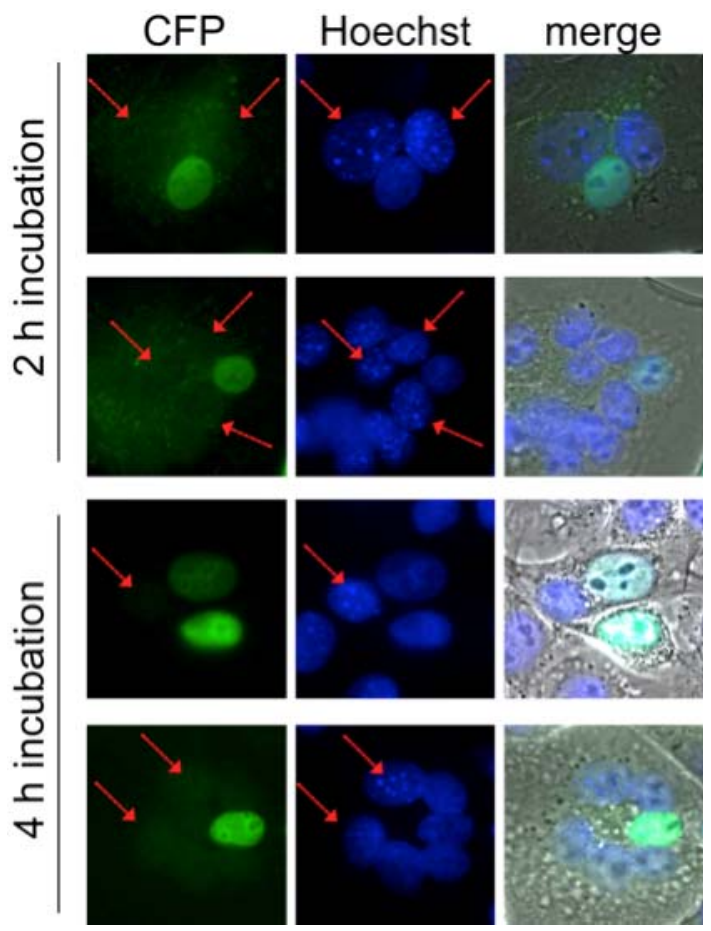

Figure 29: No shuttling of Aos1 in heterokaryon assays. Heterokaryate cells were obtained by fusion of untransfected mouse 3T3 cells with transiently transfected HeLa cells over expressing CFP-Aos1. After 2 or 4 hours of incubation DNA was stained with Hoechst (blue) and intracellular distribution of CFP-Aos1 (green) was analyzed by fluorescence microscopy. Nuclei from mouse 3T3 cells show characteristic bright heterochromatin in the Hoechst channel which are abscent from human HeLa nuclei.

these assays CFP-Aos1-expressing human cells and untransfected mouse cells were fused to heterokaryon cells containing at least one human and one murine nucleus. De novo protein biosynthesis was inhibited by the addition of cycloheximide, and the intracellular distribution of CFP-Aos1 was analyzed (Fig. 29). Since CFPAos1 was not newly synthesized, the accumulation of fluorescent signal in the blue spotted mouse nuclei (marked by arrows) would point towards shuttling of the CFPAos 1 reporter protein: In this case, Aos 1 would have been actively exported from a tranfected HeLa nucleus and subsequently re-imported into a mouse nucleus. Figure 29 demonstrates that the CFP signal did not accumulate in the mouse nuclei within 4 hours. Hence, shuttling of CFP-Aos1 was not detected.

However, interspecies heterokaryon assays only allow detection of shuttling if the levels of export are relatively high. In contrast, fluorescence microscopy techniques based on photobleaching are more sensitive methods that allowing the detection of even low levels of nucleocytoplasmic shuttling.

For this reason, I tested shuttling of CFP-Aos1 in multinuclear cells by using combined FRAP and FLIP (for details see chapter METHODS - Combined FRAP and FLIP in multinuclear cells). HeLa cells expressing the reporter protein CFP-Aos1 were fused to multinuclear cells. Then, the fluorescent signal in one of the nuclei was bleached (Fig. 30A, ROI-1, red) and images from different time points after bleaching were collected for quantification. Some selective images are shown in Fig. 30A. For 
A

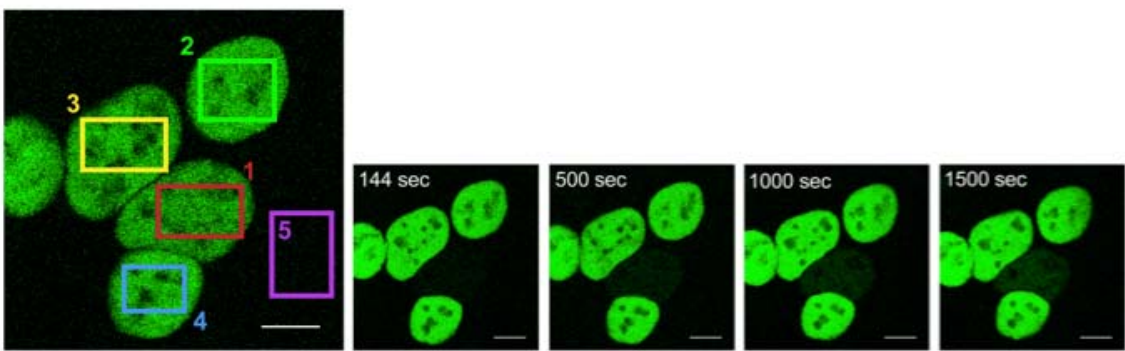

B

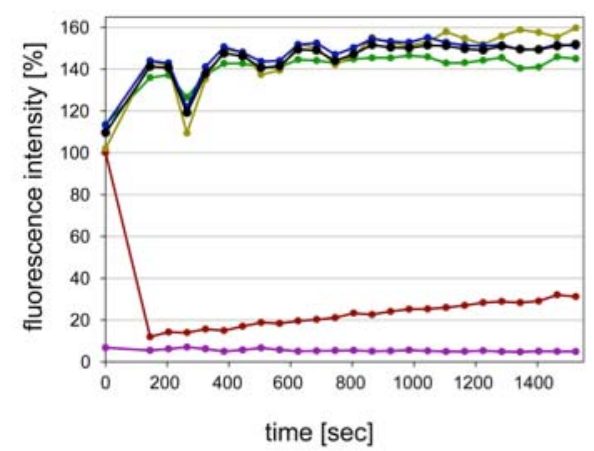

C

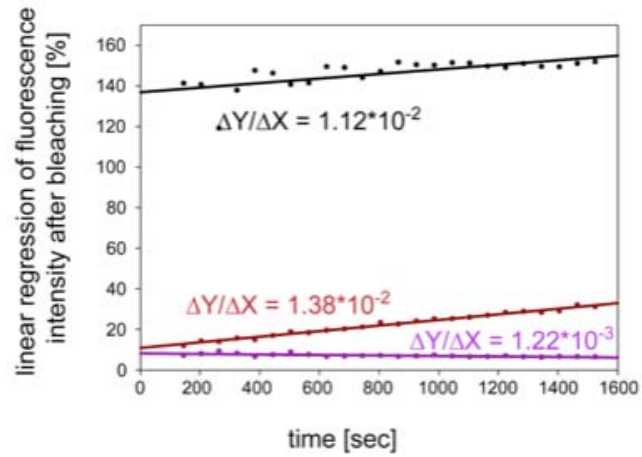

Figure 30: Combined FRAP and FLIP analysis shows no shuttling of Aos1. HeLa cells were transiently transfected with pcDNA3.1(-)-CFP-Aos1 and fused to homopolycaryons. CFP signal in one nucleus was completely bleached by multiple iteration of scanning with $100 \%$ intensity of 458 $\mathrm{nm}$ from the argon laser using a CLSM510 (Zeiss). Images were aquired at different time points and signal intensities in 5 different ROls (regions of interest) were tracked. (A) Selective images of different time points before and after bleaching. ROls used for subsequent calculations are marked as follows: bleached nucleus ROI 1 (red), unbleached neighbouring nuclei ROls $2-4$ (green, yellow, blue) and cytoplasmic background ROI 5 (pink). (B) Graphs of the fluorescent signals of the ROls $1-5$ displayed in (A), are presented in the according colours red, green, yellow, blue and pink. The average signal from ROls $2-4$ are represented by the black graph. (C) Linear regression curves of the fluorescent signals in ROI 1 (red), ROls $2-4$ (black) and ROI 5 (pink) after bleaching. The calculated slopes represent the speed of signal accumulation. Data were processed with sigma plot 8.02 (Systat Software Inc.). Bar, $10 \mu \mathrm{m}$.

data processing, the fluorescent signal in the bleached nucleus (Fig. 30A, ROI-1, red), the surrounding unbleached nuclei (ROls 2-4, green, yellow and blue) and in the unbleached cytoplasmic background (ROI-5, purple) were tracked within defined regions of interest (ROIs). Subsequently, fluorescent intensities in the ROls were calculated in percentage of the intensity of ROI 1 before bleaching (Fig. 30B). The diagrams in Fig. 30B show that after bleaching of the marked nucleus (red graph) no strong increase in the bleached (FRAP) or decrease in an unbleached nucleus (FLIP) (green, yellow, blue graphs) was detected within the range of 25 minutes. Pictures taken at later time points, after re-adjusting the focus, showed similar signal distribution (data not shown). Linear post-bleaching regression curves were calculated of the signal in the bleached nucleus (Fig. 30C, red graph), of the average 
signal from the unbleached nuclei (black graph) and from the background fluorescence (purple graph). To compare the speed of changes in the fluorescent signal, the slopes of the linear regressions corresponding to the speed were calculated. The resulting data of this analysis reveal that the fluorescent signal in bleached and unbleached nuclei increased with similar speed, whereas the background signal changed approximately 10 times slower. This result shows that no significant FRAP or FLIP occured, which would have become apparent by a faster increase in the bleached nucleus (ROI 1, red graph) compared to the unbleached nucleus (ROls 2-4, black graph) in a static low background.

Since it has been shown that the level of SUMOylation of target proteins can be altered by exposing cells to different stresses (Mao et al. 2000; Sacher et al. 2006; reviewed in Tempe et al. 2008), cytoplasmic E1 may be generated by re-localization induced by a certain stress stimulus. However, analyses of the intracellular localization of endogenous Aos1 and Uba2 after incubation at $0.04 \% \mathrm{CO}_{2}$, on ice or in the presence of $1-10 \mathrm{mM} \mathrm{H}_{2} \mathrm{O}_{2}$ did not point towards nuclear export of the $\mathrm{E} 1$ upon exposure to the tested stresses (data not shown).

Taken together, in of the performed experiments neither active export of Aos 1 nor binding of Aos1, Uba2 or E1 complex to the export receptor CRM1 was detected, suggesting that cytoplasmic pool of SUMO E1 is not generated by constant export out of the nucleus. Hence, other mechanisms such as the inhibition of import or cytoplasmic retention may account for the generation of cytoplasmic E1 (for details see chapter DISCUSSION).

\section{Analysis of the effects of mislocalized SUMO E1 in yeast}

As described in the introduction, SUMOylation takes place both in the nuclear and cytoplasmic compartment. However, SUMO E1 enzyme is barely detectable in the cytoplasm and does not appear to shuttle between these compartments. One explanation for this apparent discrepancy is that the E1 enzyme is expressed in large excess, such that the low levels in the cytoplasm are not rate limiting. Alternatively 
E1 may not be needed in a specific compartment, which is conceivable if the E2 enzyme can shuttle after beeing loaded with SUMO.

To test whether nuclear localization of E1 is required, I analyzed the effects of mislocalized SUMO E1 in yeast cells, which have the same natural E1 distribution as human cells (Dohmen et al. 1995; Azuma et al. 2001; Pichler et al. 2002). Since Aos1/Uba2 is essential in S. cerevisiae (Dohmen et al. 1995; del Olmo et al. 1997), this system allowed the generation of UBA2 shuffle strains. Shuffle strains are yeast strains in which the endogenous copy of an essential gene is deleted and replaced with a marker, while cells are kept alive by an exogenous copy of this gene. Selective loss of this rescue gene and parallel expression of a plasmid-encoded variant protein subsequently allows phenotypical analysis of the variant. By means of a UBA2 shuffle strain I aimed to analyze the cellular consequences of cytoplasmically localized Uba2 without the background of endogenous Uba2.

\subsection{Human Uba2 can not substitute for deletion of yeast Uba2 in UBA2 shuffle strains}

By introduction of a shuffle plasmid containing the UBA2 gene and disruption of the genomic UBA2 gene I generated a yeast UBA2 shuffle strain. The cells can grow on normal medium due to expression of Uba2 encoded by the shuffle plasmid, whereas 5-FOA containing medium selects for cells that have lost the shuffle plasmid. Since UBA2 is an essential gene (Dohmen et al. 1995; del Olmo et al. 1997), the created shuffle strains could not survive without the shuffle plasmid and would consequently not grow on the selctive 5-FOA medium. However, cells with an additional 5-FOA insensitive plasmid with the ORF of Uba2 would still be able to grow in the presence of 5 -FOA. 


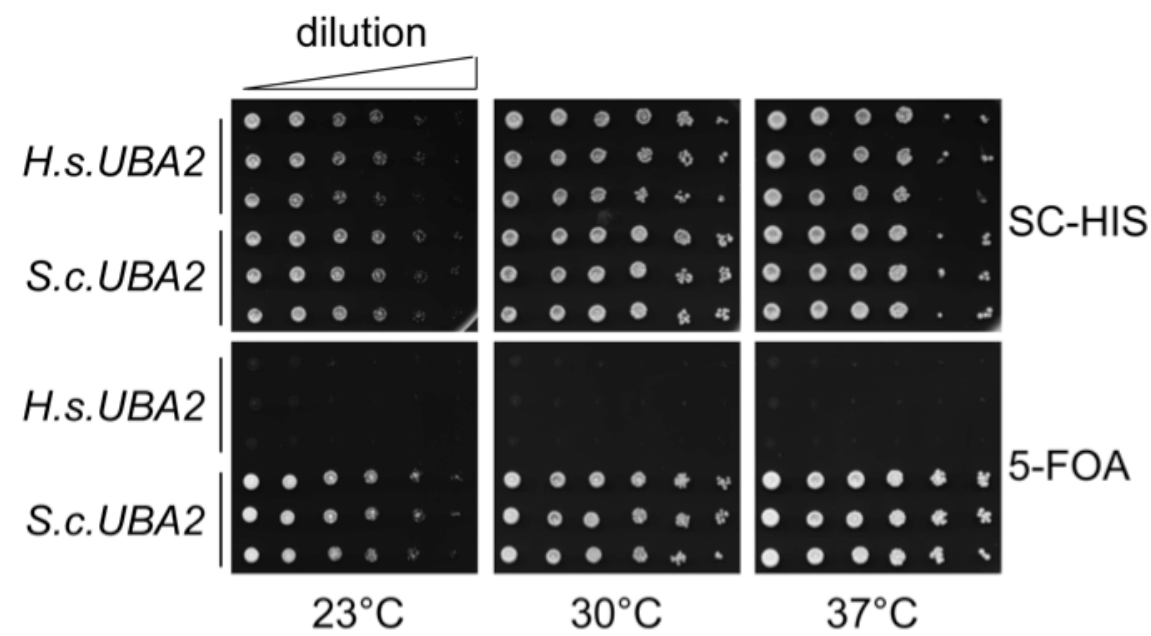

Figure 31: Expression of S.c.Uba2 but not H.s.Uba2 rescues the deletion of endogenous Uba2 in yeast. Endogenous Uba2 in yeast was replaced by expressing exogenous Uba2 in the shuffle strain ESM356-1/pRS316-UBA2/uba2A::nat. Exogenous Uba2 from S.cerevisiae or H.sapiens was overexpressed under ADH promoter from p413-expression constructs. Logarithmic growing cells with indicated genotypes were serially diluted and spotted onto plates that were incubated for 2 days at indicated temperatures. Growth on SC-HIS plates verifies equal dilutions and rescue for deletion of endogenous Uba2 was analyzed without the background of genomic Uba2 on 5-FOA plates.

To test the UBA2 shuffle strain for functionality and to test whether human Uba2 can complement yeast Uba2, either human or yeast Uba2 was mildly overexpressed under $\mathrm{ADH}$-promoter from a p413-construct that was transformed into the yeast strains (Fig. 31). Equal growth in the presence of the UBA2 shuffle plasmid on SCHIS plates demonstrated that an equal cell number of the two strains was spotted. Analysis of growth in the absence of UBA2 shuffle plasmid on 5-FOA plates showed that the cells only survived when yeast but not when human Uba2 was overexpressed from the p413-construct. These experiments showed that the shuffle strain is functional and revealed human Uba2 does not rescue disruption of the essential UBA2 gene in $S$. cerevisiae. Consequently, all following rescue experiments concerning the phenotypic analysis of Uba2 mutants had to be performed with variants of yeast Uba2.

\subsection{Predominantly nuclear localization of Uba2 is not essential for the viabilty of yeast}

To address the question whether nuclear localization of SUMO E1 is important I tested mislocalized NLS mutants of Uba2 for complementation of endogenous Uba2 
in the generated yeast shuffle strains. For this purpose, UBA2 shuffle strains expressing wild type yeast Uba2 (Uba2-wt) were compared to strains expressing the NLS mutant of yeast Uba2 (Uba2- $\Delta$ NLS) or the NLS mutant Uba2 with an additional NES (Uba2- $\Delta$ NLS-NES) to reinforce cytoplasmic localization.

A dilution

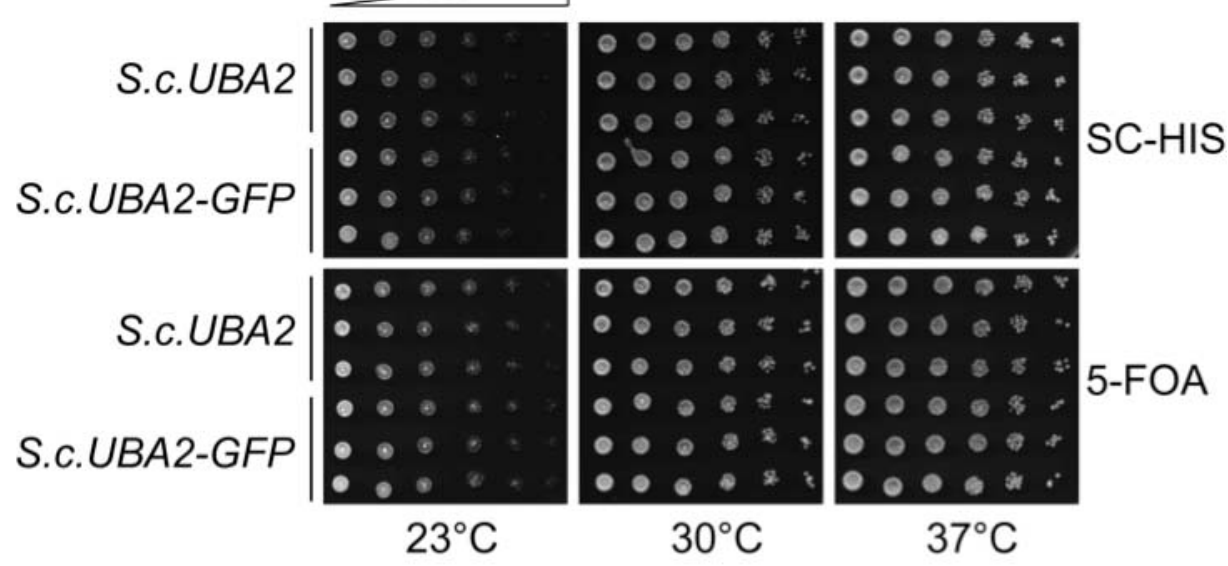

B

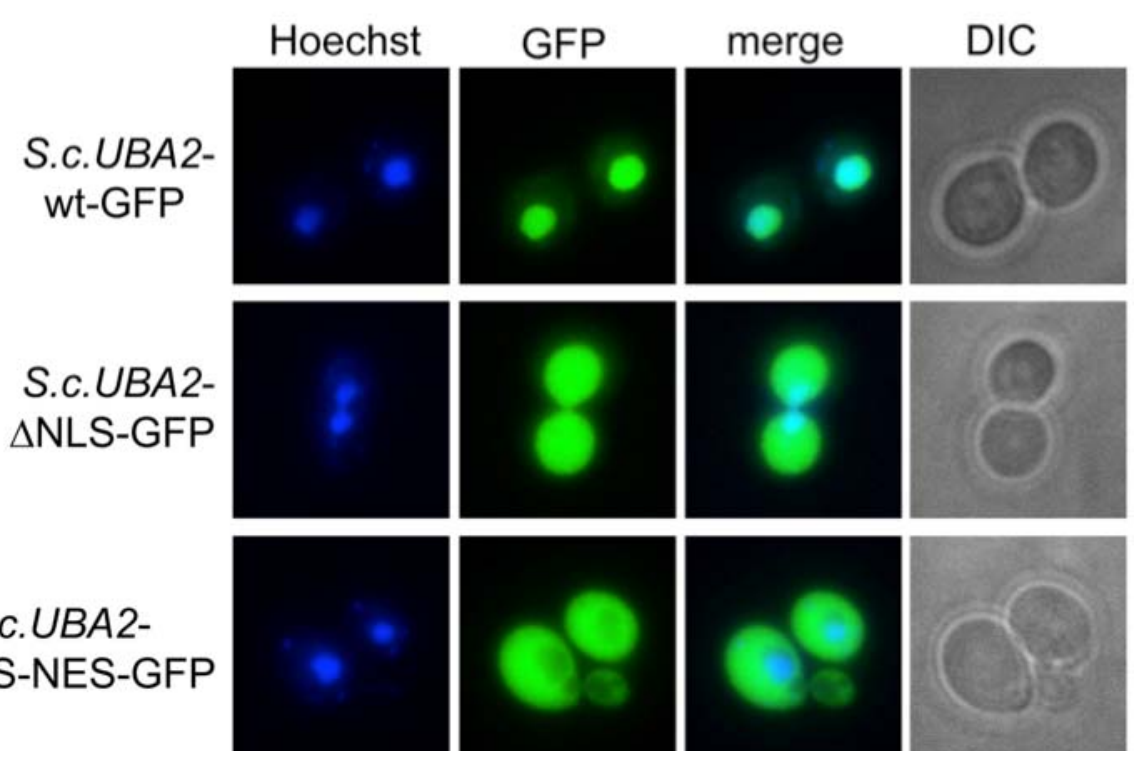

Figure 32: Localization of S.c.Uba2-GFP in UBA2 shuffle strains. (A) Influence of a C-terminal GFP-tag on the functionality of Uba2 was analyzed by comparing the rescue ability of S.c.Uba2 and S.c.Uba2-GFP in the UBA2 shuffle strain by growth on 5-FOA plates. (B) Variants of Uba2 were expressed in the shuffle strain without background of endogenous Uba2 after selection on 5FOA plates. Abbreviations accord to the following variants: wild type (wt), KRTK619-622AATA $(\triangle N L S)$ and $\triangle N L S$ with an additional NES in the C-terminus of Uba2 ( $\triangle N L S-N E S)$. Cell cultures were harvested, DNA was stained with Hoechst (blue) and intracellular distribution of Uba2-GFP (green) was analyzed by fluorescence microscopy. 
A comparison of the rescue by untagged and by C-terminally GFP-tagged yeast Uba2 (Fig. 32A) revealed that the GFP-tag, which was introduced for intracellular detection, did not alter viability of the strain. Then, fluorescence microscopic analyses of shuffle strains expressing GFP-tagged yeast Uba2 variants were performed to illustrate the intracellular localization of the variants. While the wild type protein was exclusively located in the nucleus, visualized by Hoechst staining of the DNA, the NLS mutant Uba2- $\Delta$ NLS mislocalized to the cytoplasm (Fig. 32B). However, the NLS mutant was additionally detected in the nucleus of the yeast cells. This partial nuclear localization of overexpressed Uba2- $\Delta$ NLS might be due to the fact that the NLS (according to the mapped NLS of the human homolog) was only partially mutated, and is conform with the localization of overexpressed human Uba2 NLS mutants in HeLa cells (Fig. 16). Since I aimed to analyse the phenotype of only cytoplasmically located Uba2, I created a variant that contains a mutated NLS and an additional nuclear export signal (NES). This Uba2 variant, Uba2- $\Delta$ NLS-NES, indeed mainly localized to the cytoplasm, which can be seen by the negative staining of the nucleus (Fig. 32B).

After ensuring cytoplasmic localization of the Uba2 variants, the variant strains were tested for rescue of the disruption of endogenous UBA2 (Fig. 33). All strains showed comparable growth on SC-HIS plates verifying equal numbers of spotted cells. Unlike the negative control with human Uba2, the three strains expressing differently localizing yeast Uba2 variants grew similarly on 5-FOA, indicating that the intracellular distribution of Uba2 and thereby of SUMO E1 does not influence the viability of yeast under normal conditions.

Several examples from the literature document that the level of SUMO modification can change in response to stress (Saitoh et al. 2000; Kurepa et al. 2003; Zhou et al.

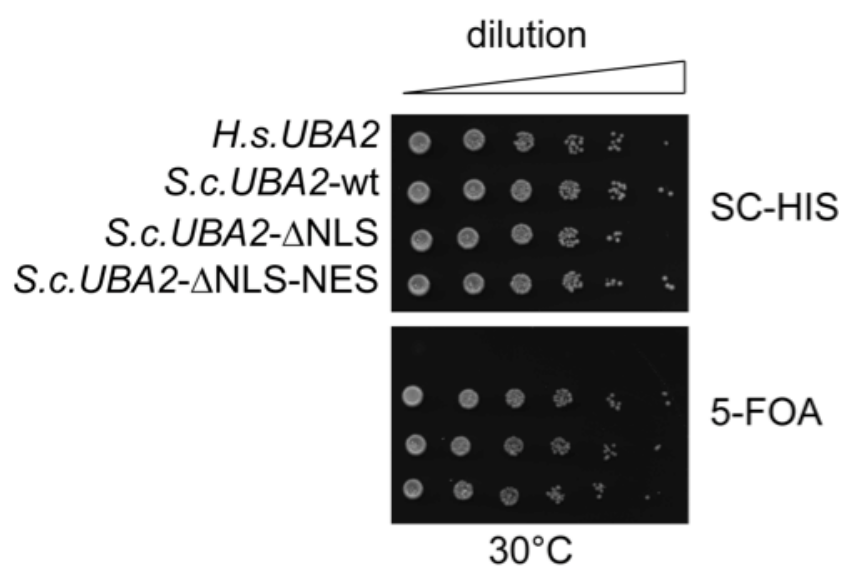

Figure 33: Cytoplasmic S.C.Uba2 rescues the deletion of endogenous Uba2 in yeast. Serial dilutions of logarithmic growing shuffle strains with indicated genotypes were spotted onto plates and incubated for 2 days at $30{ }^{\circ} \mathrm{C}$. Rescue for deletion of endogenous Uba2 was analyzed without the background of genomic Uba2 on 5-FOA plates with cells expressing H.s.Uba2 serving as negative control. 
A

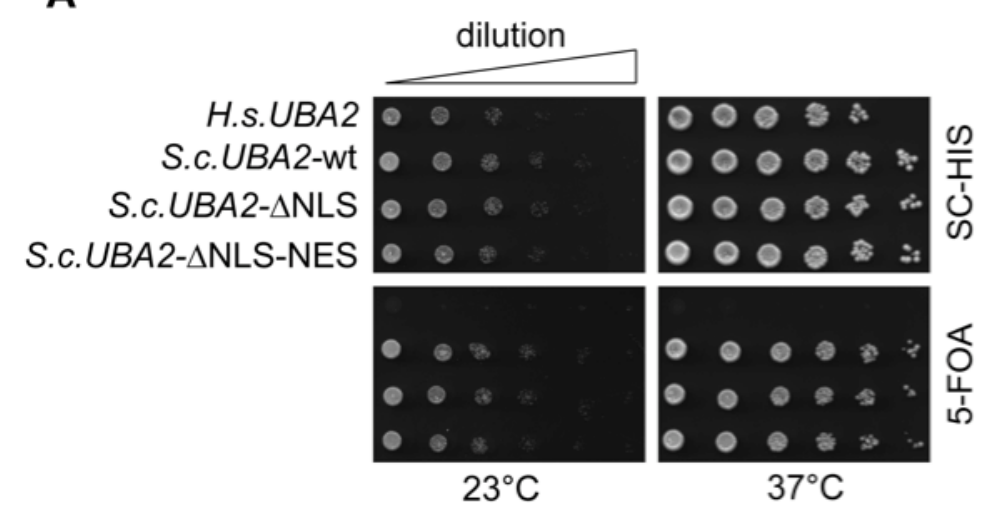

B

dilution

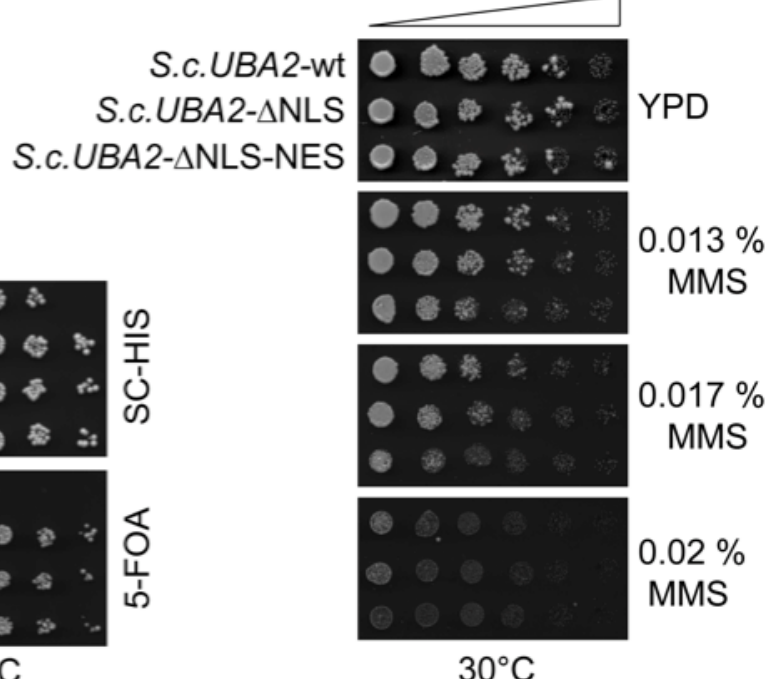

$30^{\circ} \mathrm{C}$

Figure 34: Viability of yeasts with cytoplasmically localized Uba2 is not altered by suboptimal temperatures or increased DNA damage. Serial dilutions of UBA2 shuffle strains with indicated genotypes were spottet onto plates and incubated for 2 days. Growth tests under conditions of suboptimal temperatures were performed on 5-FOA plates at 23 or $37^{\circ} \mathrm{C}(\mathrm{A})$. Growth under increased DNA damage stress was tested on YPD plates supplemented with different indicated amounts of the DNA damaging reagent MMS at $30^{\circ} \mathrm{C}(\mathrm{B})$.

2004; Golebiowski et al. 2009; reviewed in Bossis et al. 2006; Tempe et al. 2008). A very recent study of rice SUMOylation showed the accumulation of high levels of SUMO conjugates under conditions of cold stress and salt stress (Chaikam et al. 2010), suggesting an interesting role of SUMOylation in handling stress conditions. Due to the role of SUMOylation in stress-response, I wanted to test whether the viability of yeast strains exposed to stress conditions is altered when the SUMO E1 is predominantly localized in the cytoplasm. Growth of the shuffle strain expressing partially or completely cytoplasmically localizing Uba2 (S.c.UBA2- $\mathrm{NLS}$, S.c.UBA2$\triangle$ NLS-NES) was not altered compared to nuclear wild type Uba2 (S.c.UBA2-wt) when cells were subjected to conditions of suboptimal temperatures (Fig. 34A). Furthermore, the ability of the UBA2 variants to rescue disruption of endogenous UBA2 was tested under conditions of different intensities of DNA damage stress caused by growth on MMS-containing medium (Fig. 34B). Again, no drastic differences in viability of strains with nuclear versus cytoplasmic SUMO E1 were detected. Taken together, these results indicate that a predominantly nuclear localization of Uba2 and therefore of the SUMO E1 complex is not essential for the viability of Saccharomyces cerevisiae. 


\subsection{Cytoplasmic localization of Uba2 in yeast does not significantly alter the SUMOylation pattern}

Since the E1 localization had no obvious effects on the viability of yeast, the question emerged whether SUMO modification is altered at all by mislocalization of E1. Published data, based on studies with a C-terminally truncated Uba2 fragment, indicate that the overall SUMOylation pattern in crude yeast cell lysate is slightly altered (Lois et al. 2005). However, I wanted to verify whether SUMOylation in the nucleus or the cytoplasm is altered specifically by mislocalization of the E1 enzyme. To address this question, I analyzed the Smt3 (yeast SUMO) conjugation pattern in nuclear and cytosolic fractions of yeast strains expressing nuclear (S.c.UBA2-wt) or cytoplasmic localized Uba2 (S.c.UBA2- $\mathrm{NLS}$, S.c.UBA2- $\triangle \mathrm{NLS}-\mathrm{NES}$ ).

Figure 35 shows the analysis of protein equivalents of whole cell lysates (I), nuclear $(\mathrm{N})$ and cytosolic fractions $(\mathrm{C})$ of the indicated strains. The nuclear protein Nop1 (nucleolar protein 1) and the cytoplasmic protein PGK1 (phosphoglycerate kinase 1) were used as marker proteins to control for proper fractionation. Surprisingly, in 3 independent experiments, the overall level of Smt3 modified species in S.c.UBA2-wt strains were lower compared to the strains expressing the Uba2 mutants. However the reasons are still not understood. Nevertheless a longer exposure of the blot allowed to compare the Smt3 conjugation patterns.

The patterns of S.c.UBA2- $\triangle \mathrm{NLS}$ and S.C.UBA2- $\triangle \mathrm{NLS}-\mathrm{NES}$ only showed marginal differences in the intensities of the two bands at $120 \mathrm{kDa}$ and $110 \mathrm{kDa}$ in the cytosolic fraction (marked with *). The pattern of the wild type shuffle strain also showed low intensity of these two bands and furthermore completely lacked a $35 \mathrm{kDa}$ band (marked with \#) present in all fractions of the mutant strains. Besides these differences the overall ratio of Smt3-modified species in nuclear versus cytoplasmic fractions was similar in the tested strains, confirming findings from previous studies with C-terminally truncated Uba2 by Lois et al. (Lois et al. 2005).

Altogether, these results indicate that the localization of the SUMO E1 does not dramatically influence SUMOylation in the nucleus or in the cytoplasm. Since immunoblotting only allows detection of abundant SUMO targets, mass spectrometry analysis will be required for complete elucidation of effects caused on the level of individual targets.

How can it be that SUMOylation in the nucleus and in the cytoplasm is independent of the localization of the essential E1 enzyme? One attractive hypothesis is that the 
SUMO E2 enzyme Ubc9 shuttles between both compartments in its free and in its thioester-charged form (for details see chapter DISCUSSION). This will be a topic for future investigations.

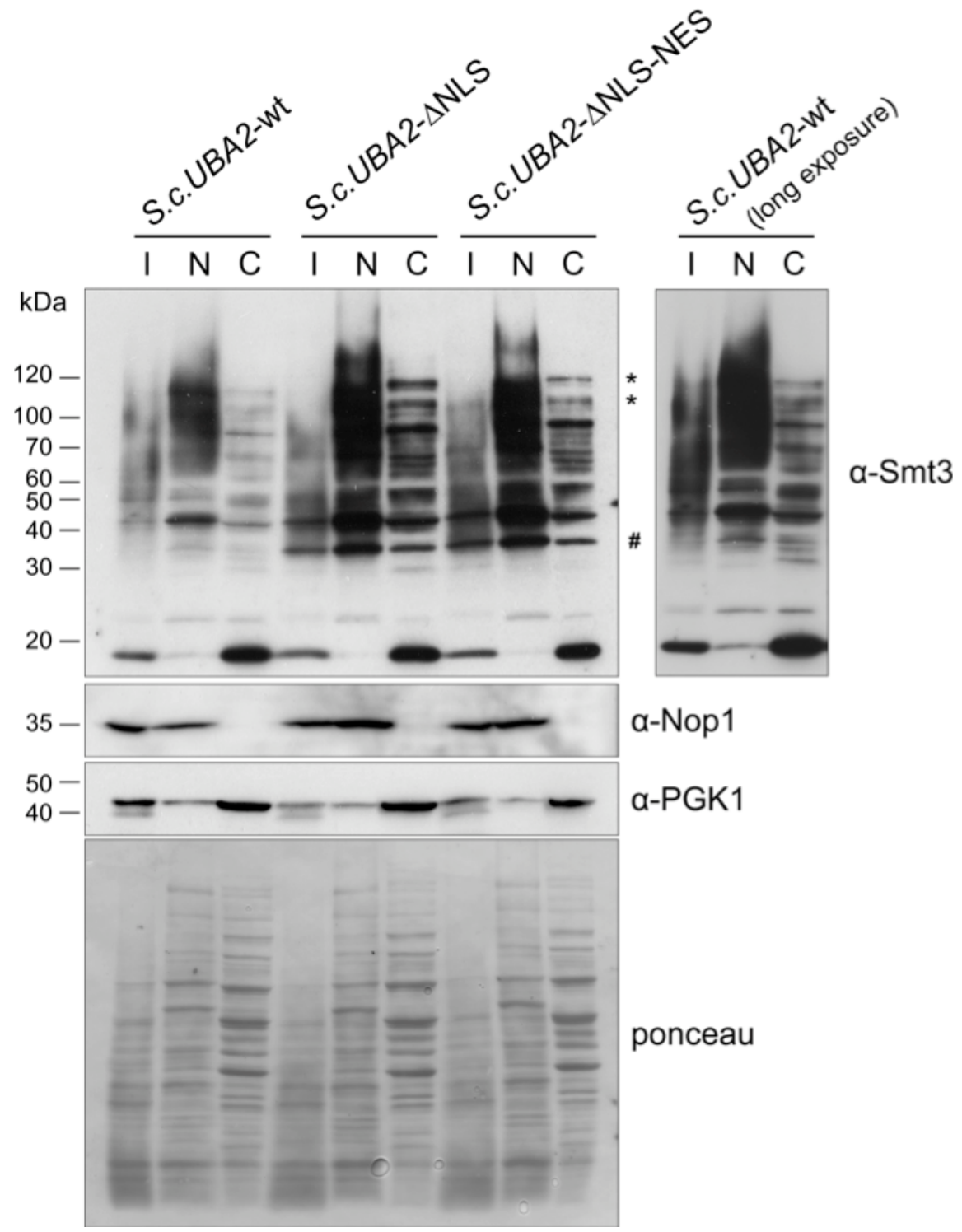

Figure 35: No significant changes in SUMO-pattern of nuclear and cytosolic fractions upon cytoplasmic localization of Uba2 in yeast. Cultures of UBA2 shuffle strains with indicated genotypes were harvested and treated with zymosan. Resulting spheroblasts were homogenized and fractionated into nuclear and cytosolic fractions by centrifugation. Protein equivalents of input (I), nuclear (N) and cytosolic (C) fractions were subjected to SDS PAGE and analyzed by immunoblotting with $\alpha$-Smt3, $\alpha$-Nop1 or $\alpha$-PGK1 antibody. A longer exposure of the wild type samples from the same blot allows better comparison. 


\section{DISCUSSION}

The heterodimeric Aos1/Uba2 complex is to date the only enzyme which is known to activate SUMO proteins. The work presented in this thesis aims to better understand the mechanisms that underlie the distinct intracellular localization of Aos1 and Uba2. Consistent with previous data I could demonstrate that the E1 complex predominantly localizes to the nucleus of cells whereas the cytoplasmic fraction of the enzyme is very small. The separation of the cellular SUMO E1 into a cytoplasmic and a nuclear pool served as a starting point to characterize the molecular mechanisms underlying the generation of these pools. In my studies I focused on the characterization of the nuclear import, which led to the identification of two ways of generating nuclear E1 complex. Additional investigations on potential nuclear export of the enzyme indicated that there is no frequent exchange between the two E1 pools. In combination with the fact that the cytoplasmic pool of Aos1 and Uba2 is very small, this led to the conclusion that the cytoplasmic SUMO activating activity is constantly low raising the question whether the E1's intracellular localization is at all important for SUMOylation in different cellular compartments.

In the following section some important details and interesting aspects arising from the presented results will be discussed in more detail.

\section{Aos1 contains a c-Myc like NLS}

Mutational analysis of the E1 subunit of Aos1 revealed that nuclear accumulation of Aos 1 requires a cluster of three basic amino acids ${ }^{195} \mathrm{KRAK}^{198}$ (Fig. 12, upper lane). This essential cluster exactly matches the consensus sequence K-K/R-X-K/R of classical monopartite NLSs (Chelsky et al. 1989). Interestingly, further analysis of Aos1 revealed that the adjacent residues Leu-199 and Asp-200 also participate in the import of Aos1 (Fig. 12, upper lane). Hence, the identified NLS of Aos1 shares most residues of the conserved motif PxxKRxKLD of the unconventional NLS of the oncoprotein C-Myc $\left({ }^{320}\right.$ PAAKRVKLD $\left.{ }^{328}\right)$ (Stone et al. 1987; Dang et al. 1988). While Pro-320 in the c-Myc NLS has been shown to be required for nuclear accumulation (Makkerh et al. 1996), the substitution of the corresponding Pro-192 in the sequence of Aos1 ( ${ }^{192}$ PDTKRAK ${ }^{198}$ ) did not alter Aos1's intracellular distribution. An explanation for the apparent discrepancy could be varying roles of the corresponding prolines in 
the two motifs: The Pro-320 in c-Myc might be a necessary element to ensure structural flexibility required for the interaction with importin $\alpha$, whereas in Aos 1 this structural function might simply be carried out by other residues. In line with this hypothesis, crystallographic analysis by the laboratory of John Kuriyan revealed that the main interactions between the c-Myc NLS peptide $\left({ }^{319}\right.$ GPAAKRVKLDS $\left.{ }^{329}\right)$ and importin $\alpha$ occur via the cluster ${ }^{323} \mathrm{KRVKL}^{327}$ (Conti et al. 2000). This motif binds in an extended conformation to the binding pockets of the large binding site of importin $\alpha$ forming the maximal number of hydrogen bonds possible and a number of salt bridges. In contrast, the hydrophobic N-terminal residues of c-Myc's NLS $\left({ }^{320} \mathrm{PAA}^{322}\right)$ are only anchored by a single hydrogen bond and are therefore likely to not mediate crucial interactions. Regarding Aos1, the crystal structure of SUMO E1 complex revealed that the identified NLS $\left({ }^{195} \mathrm{KRAKLD}^{200}\right)$ is located in a flexible loop (Lois et al. 2005). Due to insufficient electron density, the loop between Thr-179 and Glu-203 is missing in the solved structure of Aos1, indicating that the loop has a high structural flexibility. Since the loop already begins more than 10 amino acids $\mathrm{N}$ terminal of the NLS, the flexibility may depend on structural elements other than Pro192.

Altogether, the data from crystallographic analyses of the NLSs of c-Myc and of Aos1 support the suggestion that the prolines adjacent to the basic cores of the NLSs probably have different functions. It would be interesting to test whether an intermolecular exchange of the NLSs in c-Myc and Aos1 would result in an altered sensitivity of the nuclear import of the proteins towards mutation of the respective proline residues.

\section{Nuclear E1 is generated in two ways}

The characterization of the nuclear import of SUMO E1 resulted in a model schematically illustrated in Figure 21. According to this model active nuclear E1 can be generated in two ways: One possibility is the independent import of the single subunits via their distinct import signals by importin $\alpha / \beta$ and subsequent assembly of active Aos1/Uba2 complex inside the nucleus (Fig. 21, left). The second mechanism allows the import of pre-assembled E1 complex via the NLS of Uba2, which is also mediated by heterodimeric importin $\alpha / \beta$ (right). 
Nuclear import of Aos1 and Uba2 is required in two scenarios, after de novo protein biosynthesis and after breakdown and reformation of the nucleus during mitosis. The model for E1 import allows for a solution for both situations: After de novo synthesis of the subunits in the cytoplasm Aos1 and Uba2 could be imported independently by their distinct NLSs followed by E1 complex formation. On the other hand, re-import of already assembled E1 complex after mitosis can occur via the NLS of Uba2 and thereby makes disassembly before import and reassembly after import unnecessary. Biochemical and cell biological analyses revealed that the interaction of importin $\alpha / \beta$ with already formed E1 complex occurs exclusively via the NLS of Uba2, whereas the NLS of Aos1 does not mediate importin-E1 interactions. This observation may be explained by altered accessibilities of the NLSs in the single subunits compared to assembled E1 complex. The crystal structure of the Aos1/Uba2 complex, solved by the laboratory of Chris Lima allows comparing the spatial positions of the NLSs of Aos1 and of Uba2 (Fig. 36) (Lois et al. 2005):

In the case of Uba2 the whole C-terminal domain from amino acid 551 on has not been solved in the structure, which is why the closest residue Ala-550 must serve as a marker for the approximate position of this domain. Fig. 36A gives a rough idea of the potentially exposed position of the missing domain adjacent to the UbL-domain of Uba2. The relatively long distance of this domain from the interaction sites of Aos 1

A

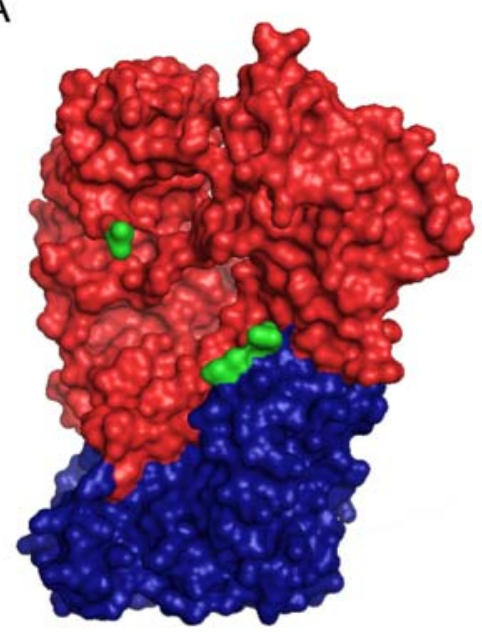

B

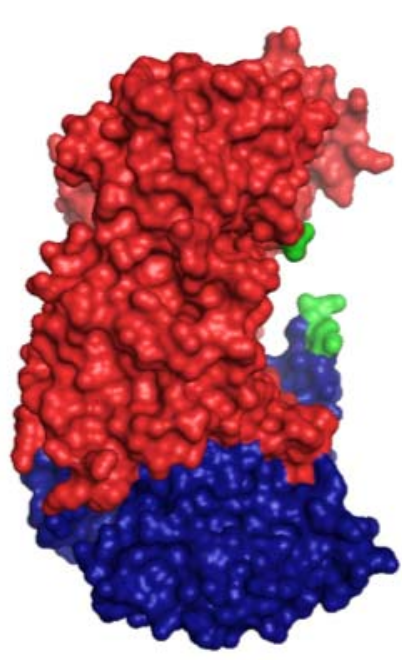

Figure 36: Crystal structure of Aos1 and Uba2. Illustration of the crystal structure of heterodimeric Aos1/Uba2 complex solved by Lois et al. (Lois et al. 2005). Aos1 is coloured in blue with Thr-179 and Glu-223, which flank the missing loop with the identified NLS, marked in green. Uba2 is shown in red and the C-terminal Ala-550 of the solved Uba2 fragment highlighted in green. $(A)$ and $(B)$ represent different views on the same complex. The raw data were obtained from the Protein Data Bank (PDB) and images were generated with PyMOL v0.99 (DeLano Scientific LLC). 
and Uba2 suggests that the accessibility of Uba2's NLS is probably not altered by complex formation.

In the case of Aos1, the loop in which the NLS is located was also too flexible for detection. Therefore, the adjacent amino acids Thr-179 and Glu-223 indicate the position of the loop (Fig. 36B). The structure of the E1 complex shows that the NLS of Aos1 extends into a cavity-like topology formed by both subunits. In fact, this hidden position of the Aos1 NLS Aos1 is even more drastic than displayed since the missing domain of Uba2 is just above this region of Aos1. Due to this direct surrounding of the Aos1 NLS by Uba2, it is obvious, that formation of the E1 complex causes masking of the NLS of Aos1.

Consequently, the accessible position of the Uba2 NLS and the hidden localization of the Aos1 NLS in assembled E1 complex provide a likely explanation why only the Uba2 NLS is capable of mediating interaction of formed SUMO E1 complex with importin $\alpha / \beta$.

\section{How is the cytoplasmic E1 pool generated?}

Immunofluorescent analyses by epi- and confocal fluorescence microscopy indicate that Aos1 and Uba2 are almost exclusively located in the nuclei of cells (Fig. 22) (Dohmen et al. 1995; Azuma et al. 2001; Pichler et al. 2002; Pichler et al. 2004). However, the analysis of subcellular fractions of HeLa cells and hippocampal neurons performed within this work, in line with fractionation of RAW264.7 macrophages by Dr. Guillaume Bossis (Bossis et al. 2006), emphasize that a portion of Aos1 and Uba2 is located in the cytoplasm of cells. The existence of a cytoplasmic SUMO E1 pool immediately raises the question how this pool is generated.

Analogous to its nuclear import, Aos1 and Uba2 could be exported from the nucleus into the cytoplasm. Since data obtained within this work indicate that active export via the main export receptor CRM1 does most likely not occur, I also investigated the general behaviour of Aos1 regarding nucleocytoplasmic shuttling. The applied method of combined FRAP and FLIP in homopolykaryons is a state-of-art method for the analysis of a slow shuttling reporter construct. However, in addition to the lack of a positive control for the performed experiment, the experimental setup itself was not well suited to analyze E1 shuttling: The assumption that most cellular Aos1 and Uba2 exists in form of a stable heterodimeric complex suggests that potential shuttling 
would occur as heterodimeric Aos1/Uba2 complex. For this reason the experiment should be set up such that potential shuttling of E1 complex can be determined. In the performed experiment this was not ensured. Since Aos1 alone is rather stable, the performed experiment with the overexpressed CFP-Aos1 reporter protein served to analyze single Aos1 rather than the Aos1/Uba2 complex. In contrast to monomeric Aos1, single Uba2 has been shown to be strongly degraded, whereas complex formation with Aos1 protects it from degradation (Boggio et al. 2007). Therefore, expression of Uba2 likely results in low levels of expressed Uba2, which would mostly exist in a complex with Aos1. Consequently, instead of CFP-Aos1, an UbaYFP reporter construct or co-expression of both subunits would be more suited for FRAP/FLIP experiments to find out if the E1 complex shuttles between nucleus and cytoplasm.

Inhibition of nuclear import by posttranslational modifications displays an additional potential mechanism to generate cytoplasmic SUMO E1. Selective inhibition of nuclear import of target proteins could for example result from phosphorylation of residues adjacent to NLSs which have been shown to decrease the affinity for importin $\alpha$ (Kaffman et al. 1999; Harreman et al. 2004). Since the Uba2 NLS is required for nuclear import of SUMO E1 complex, masking of the Uba2 NLS would be sufficient to keep active E1 in the cytoplasmic compartment. And indeed, both basic clusters of the Uba2 NLS contain adjacent serine residues that could potentially be phosphorylated. In addition, SUMOylation itself presents another intriguing possibility to regulate the localization of the E1 enzyme. Interestingly, the lysines 623 and 630 of Uba2, which are located in (Lys-623) or adjacent (Lys-630) to the second cluster of the identified NLS, have been shown to function as a SUMO acceptor site in vitro (Hsiao et al. 2009). This finding immediately raises the question whether SUMOylation itself might influence the intracellular distribution of the E1 enzyme. Hence, it would be of special interest to investigate the posttranslational modification of Aos1 and Uba2, especially their cytoplasmic pools, in detail.

An alternative mechanism for potential cytoplasmic retention of $E 1$ could be specific interactions with proteins that recruit E1 complex to the cytoplasm. Interaction of cytoplasmically localized proteins with Aos1 or Uba2 could, due to the high stability of assembled E1 complex, retain the whole SUMO E1 complex in the cytoplasm. Potential candidates could for example be the Rab6-interacting protein 2 (ELKS) that has previously been shown to interact with Aos1 (K. Chmielarska, PhD thesis) or the 
partially cytoplasmic protein DJ-1 (PARK7), which interacted with Uba2 in a yeast two-hybrid screen by Eunsung Junn and his coworkers (Junn et al. 2005). However, co-immunoprecipitation experiments performed within this work failed to confirm the potential interaction of DJ-1 and Uba2 (data not shown). For the identification of new interaction partners of the SUMO E1 I set up yeast two-hybrid screens with full length Aos1 and full length Uba2 as baits. Unfortunately, the screens carried out by Dr. Manfred Kögl from the Genomics and Proteomics Core Facilities of the DKFZ in Heidelberg did not result in any potential interaction partner for Aos1 or Uba2 (data not shown).

Altogether, neither previous data nor analyses performed within this work give any indication which mechanism underlies the constitution of cytoplasmic SUMO E1. Consequently, the question how cytoplasmic $\mathrm{E} 1$ enzyme is generated remains open.

\section{Is the E1's intracellular distribution subjected to regulation?}

The regulation of SUMOylation on the level of the enzymatic machinery has to date, unlike regulation on the level of individual targets, hardly been characterized. Potential mechanisms could alter the activity, abundance or localization of SUMO enzymes and thereby regulate SUMOylation in total (reviewed in Bossis et al. 2006). Various cellular stresses like heat shock (Saitoh et al. 2000), osmotic stress (Kurepa et al. 2003), oxidative stress (Zhou et al. 2004; Bossis et al. 2006) or genotoxic stress (Mao et al. 2000; Mo et al. 2002; Park et al. 2005; Wang et al. 2005; Sacher et al. 2006) as well as hibernation (Lee et al. 2007) have already been shown to generally affect SUMOylation (reviewed in Tempe et al. 2008).

Regarding the E1 enzyme, altered abundance of Aos1/Uba2 by the CELO adenovirus (Boggio et al. 2005) and altered activity induced by oxidative stress (Bossis et al. 2006) have been reported to decrease overall SUMOylation. In addition, previous studies on the intracellular localization of the Uba2 homolog from Drosophila melanogaster revealed drastic changes in the localization of D.m.Uba2 in different developmental stages (Donaghue et al. 2001; Shih et al. 2002). Whereas Uba2 is initially distributed throughout the whole cell it has been shown to gradually enrich in the nucleus during embryogenesis. Considering the fact that most human Uba2 exists in form of assembled E1 complex (Azuma et al. 2001), this finding points towards an interesting and so far unidentified regulation of the intracellular 
localization of Aos1/Uba2. Besides this developmental change of the intracellular distribution, additional mechanisms may exist that also affect the localization of Aos1/Uba2 in non-deviding cells.

Different localization of the SUMO E1 could for example be triggered by a specific stimulus like cellular stresses, some of which have been shown to inhibit classical nuclear import pathways (Stochaj et al. 2000; Kodiha et al. 2009). However, preliminary analysis of the effect of $\mathrm{H}_{2} \mathrm{O}_{2}$ suggests that oxidative stress does not influence the distribution of Aos1 or Uba2 (data not shown).

Alternatively, intracellular localization could also be a cell type-specific phenomenon. In line with a regulatory extranuclear role of SUMOylation in neuronal cells (Martin et al. 2007), Aos1 and Uba2 are found in the cytosolic synaptosomal fraction of hippocampal neurons (Fig. 24). Unfortunately, further analysis of the localization of Uba2 in tissue slides of murine brain did not allow determining precisely the intracellular localization of Uba2 (Fig. 25).

In conclusion, changes in the localization of Uba2 during embryogenesis of Drosophila melanogaster demonstrate that regulation of the distribution of Aos1/Uba2 exists. However, it remains to be elucidated whether the localization of E1 complex is subjected to additional regulations and which molecular mechanisms underly the regulated localization of the SUMO E1 observed in fruit fly.

\section{Can the localization of Aos1 and Uba2 be seperately regulated?}

In contrast to the monomeric ubiquitin E1 the SUMO E1 is a heterodimeric complex. This dimeric composition easily seduces to speculations about seperate regulations or even additional functions of the two subunits Aos1 and Uba2. Previous studies by the laboratory of Mary Dasso revealed differences in the levels of Aos1 during cell cycle, pointing towards a seperate regulation of the abundance of the two subunits (Azuma et al. 2001). Yet, differential regulation of the localizations or even additional functions of the single SUMO E1 subunits have to date not been reported. However, the identification of distinct functional NLSs in both Aos1 and Uba2 offers the possibility of seperate regulations of the intracellular localization of the subunits. A prerequisite for different localizations of the subunits would be the availability of the proteins as single subunits. Microinjection experiments with assembled E1 complex performed within this work documents a high stability of the Aos1/Uba2 complex (Fig. 
17B). E1 composed of wild type Aos1 and NLS mutant Uba2 did not accumulate in the nucleus after microinjection in the cytoplasm of cells. Since dissociation of the E1 complex would have resulted in import of the wild type Aos1 subunit, this result indicates that the Aos1/Uba2 heterodimer is a stable complex in the environment of a living cell. Additionally, gel filtration analysis of cell extracts revealed that Aos 1 and Uba2 co-migrate in a complex, demonstrating that the vast majority of both subunits exists in form of assembled E1 complex (Azuma et al. 2001). The conclusion that most cellular Aos1 and Uba2 exist in form of a stable heterodimeric E1 complex reasons that the accessibility of Aos1 and Uba2 as single subunits is very limited. Hence, interactions of single Aos1 or Uba2 with other proteins that could perhaps seperately regulate their localization or regulate other additional functions are rather unlikely. However, it remains possible that interactions with other proteins might induce the dissociation of the E1 complex and thereby enable so far unknown regulations or functions of the SUMO E1 subunits.

\section{Is cytoplasmic E1 a prerequisite for cytoplasmic SUMOylation?}

The amount of E1 in the cytoplasm is usually very small compared to the nuclear pool. In light of the fact that SUMOylation is an essential cellular process, the differences in the levels of E1 enzyme raised the fundamental question, whether the E1's intracellular distribution is important for cells. Possibly, the vast amount in the nucleus may be needed for nuclear SUMOylation or cells may only tolerate low amounts of E1 in the cytoplasm to avoid unspecific SUMOylation.

A previous study by the laboratory of Dohmen on the Uba2 homolog from Saccharomyces cerevisiae revealed that a fragment of S.c.Uba2 ${ }_{1-554}$ lacking 82 amino acids at the C-terminus, overexpressed under Gal1-promoter, is able to rescue the deletion of endogenous $U B A 2$, even though the fragment mislocalized to the cytoplasm (Dohmen et al. 1995). A more sensitive phenotypic analysis of the S.c.Uba2 ${ }_{1-602}$ fragment under the control of the natural UBA2 promoter was performed in the laboratory of Lima. Expression of the fragment that only lacked the last 34 residues of S.c.Uba2 (including both basic clusters according to the mapped NLS in human Uba2) fully rescued the deletion of endogenous UBA2 gene but resulted in slightly altered Smt3 conjugation pattern of cell lysate (Lois et al. 2005). In contrast to these studies, Del Olmo and his co-workers observed that partial 
disruption of the UBA2 gene, resulting in S.c.Uba2 ${ }_{1-594}$ - a fragment lacking 42 Cterminal residues, leads to a 2 fold slower growth and larger cells compared to wild type yeast strains (del Olmo et al. 1997).

In line with the previous findings by Dohmen and Lima, the data obtained with the NLS-mutant S.c.Uba2- $\Delta$ NLS strongly suggest that a predominant cytoplasmic localization of the $\mathrm{E} 1$ does not alter the viability of yeast under normal or stress conditions (Fig. 33, 34). However, even the variant S.c.Uba2- $\Delta$ NLS-NES, which contained a disrupted NLS and an additional NES, was partially detected in the nucleus of cells (Fig. 32). In line with this finding, the study of Dohmen demonstrated that the C-terminal deletion fragment of S.c.Uba2, which lacked both basic clusters corresponding to the mapped NLS in H.s.Uba2, localized to both nucleus and cytoplasm (Dohmen et al. 1995). Therefore it can be assumed, that a partial nuclear localization of Uba2 very likely also underlied the studies by Lima and Del Olmo, in which the intracellular localization of the Uba2 was not explicitly tested (del Olmo et al. 1997; Lois et al. 2005). Consequently, the residual nuclear amount of E1 enzyme may explain the consistent findings that yeast strains with predominantly cytoplasmic Uba2 were viable and had no significant changes in the Smt3-modification pattern. To study the effect of E1 solely present in the cytoplasm, I already began cloning of an Uba2 variant with a cytoplasmic membrane anchor. Such a variant would exclude the localization of any residual E1 in the nucleus and would therefore allow the phenotypic analysis of exclusively cytoplasmically localized E1.

If it proves true that the intracellular localization of SUMO E1 does indeed not significantly influence SUMOylation in the nucleus or in the cytoplasm, what does this mean for the mechanisms underlying SUMOylation in these two compartments? In the case of exclusively nuclear localized E1 (scenario A), SUMO-charged E2 enzyme Ubc9 would have to be exported into the cytoplasm to provide sufficient SUMOylating activity for modification of cytoplasmic targets (Fig. 37). Subsequently, the empty Ubc9 would be imported into the nucleus for another round of loading with SUMO by the nuclear localized E1. In the artificially created situation of exclusively cytoplasmic localized E1 (scenario B; tested in yeast strains), Ubc9 would have to be loaded in the cytoplasm and be imported into the nucleus for ensuring sufficent SUMO modifying activity in the nuclear compartment. After transfer of SUMO to a nuclear target, the empty Ubc9 would be exported to the cytoplasm for another round of SUMO transfer. If it proves true that both scenarios $A$ and $B$ do not cause drastic 
changes of SUMOylation in either compartment, it could be deduced that both uncharged Ubc9 as well as SUMO-charged Ubc9 can shuttle in and out of the nucleus. This attractive model has some precedence, as ubiquitin was reported to serve as an import signal for the class III ubiquitin E2 enzyme UbcM2 (Plafker et al. 2004). Nuclear import of UbcM2 and interaction with its import receptor importin 11 were shown to depend on the charging of UbcM2 with ubiquitin.

Interestingly, the transport receptor importin 13 that has been shown to mediate nuclear import of Ubc9 has also been reported to mediate nuclear export of the eukaryotic initiation factor elF1A (Mingot et al. 2001). This unusual double function would make importin 13 a potential candidate to mediate shuttling of Ubc9. However, since the presence of RanGTP has been shown to interfere with Ubc9/importin 13 complex formation, an alternative mechanism at least for the export of empty Ubc9 would be likely. Furthermore it would be interesting to analyze whether and how the according transport receptor(s) can distinguish between the empty and SUMOcharged form of Ubc9.

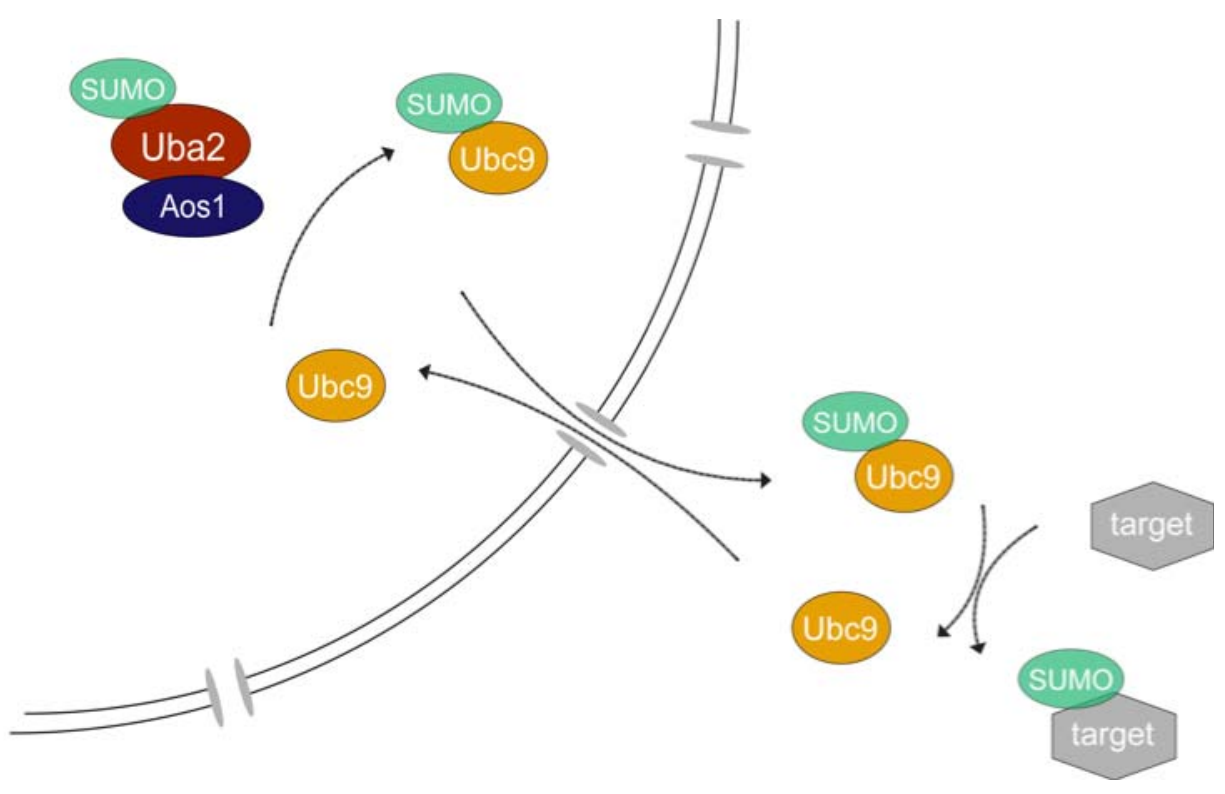

Figure 37: Working model of the nucleocytoplasmic transport of Ubc9. In the case of exclusively nuclear E1 the E2 Ubc9 is loaded with SUMO inside of the nucleus. Ubc9-SUMO is exported into the cytoplasm where it is required for SUMOylation of cytoplasmic targets. Subsequently, empty Ubc9 is imported to be again loaded with SUMO for another round of SUMOylation. 


\section{Perspectives}

Generally, the SUMO E1 enzyme Aos1/Uba2 is unequally distributed between the nuclear and the cytoplasmic compartment. The presented work provides a detailed picture of the generation of the major nuclear pool. However, intensive studies will be needed to clarify the potential roles of active nuclear export, inhibition of nuclear import or cytoplasmic retention in the generation of the cytoplasmic pool of Aos1/Uba2.

Interestingly, preliminary results indicate that the intracellular localization of Aos1/Uba2 might not be of key importance for SUMOylation, raising the possibility that Ubc9 may play a critical role providing activated SUMO in different cellular compartments. If this proves true, it will be very interesting to further investigate the mechanisms underlying the intracellular localization of the E2 Ubc9. Here, it would be particularly interesting to study the mechanisms underlying SUMOylation of targets in the plasma membrane of synapses. The long distance from the nucleus, where most E1 and E2 is located, points towards an additional yet unknown mechanism that assures the local presence of sufficient SUMOylating activity. There, detailed analysis will be needed to elucidate whether cell type-specific or signal-triggered differences in the intracellular localization of the SUMO E1 and E2 enyzems exist. 


\section{REFERENCES}

Adam, S. A., Marr, R. S. and Gerace, L. (1990). "Nuclear protein import in permeabilized mammalian cells requires soluble cytoplasmic factors." J Cell Biol 111(3): 807-16.

Amerik, A. Y. and Hochstrasser, M. (2004). "Mechanism and function of deubiquitinating enzymes." Biochim Biophys Acta 1695(1-3): 189-207.

Anckar, J., Hietakangas, V., Denessiouk, K., Thiele, D. J., et al. (2006). "Inhibition of DNA binding by differential sumoylation of heat shock factors." Mol Cell Biol 26(3): 955-64.

Andrews, E. A., Palecek, J., Sergeant, J., Taylor, E., et al. (2005). "Nse2, a component of the Smc5-6 complex, is a SUMO ligase required for the response to DNA damage." Mol Cell Biol 25(1): 185-96.

Arts, G. J., Fornerod, M. and Mattaj, I. W. (1998). "Identification of a nuclear export receptor for tRNA." Curr Biol 8(6): 305-14.

Askjaer, P., Bachi, A., Wilm, M., Bischoff, F. R., et al. (1999). "RanGTP-regulated interactions of CRM1 with nucleoporins and a shuttling DEAD-box helicase." Mol Cell Biol 19(9): 6276-85.

Azuma, Y., Tan, S. H., Cavenagh, M. M., Ainsztein, A. M., et al. (2001). "Expression and regulation of the mammalian SUMO-1 E1 enzyme." FASEB J 15(10): 1825-7.

Baake, M., Bauerle, M., Doenecke, D. and Albig, W. (2001). "Core histones and linker histones are imported into the nucleus by different pathways." Eur J Cell Biol 80(11): 669-77.

Baba, D., Maita, N., Jee, J. G., Uchimura, Y., et al. (2005). "Crystal structure of thymine DNA glycosylase conjugated to SUMO-1." Nature 435(7044): 979-82.

Baboshina, O. V. and Haas, A. L. (1996). "Novel multiubiquitin chain linkages catalyzed by the conjugating enzymes E2EPF and RAD6 are recognized by 26 S proteasome subunit 5." J Biol Chem 271(5): 2823-31.

Bailey, D. and O'Hare, P. (2004). "Characterization of the localization and proteolytic activity of the SUMO-specific protease, SENP1." J Biol Chem 279(1): 692-703.

Bayer, P., Arndt, A., Metzger, S., Mahajan, R., et al. (1998). "Structure determination of the small ubiquitin-related modifier SUMO-1." J Mol Biol 280(2): 275-86. 
Belaya, K., Tollervey, D. and Kos, M. (2006). "FLIPing heterokaryons to analyze nucleo-cytoplasmic shuttling of yeast proteins." RNA 12(5): 921-30.

Ben-Saadon, R., Zaaroor, D., Ziv, T. and Ciechanover, A. (2006). "The polycomb protein Ring1B generates self atypical mixed ubiquitin chains required for its in vitro histone H2A ligase activity." Mol Cell 24(5): 701-11.

Benson, M. D., Li, Q. J., Kieckhafer, K., Dudek, D., et al. (2007). "SUMO modification regulates inactivation of the voltage-gated potassium channel Kv1.5." Proc Natl Acad Sci U S A 104(6): 1805-10.

Bergink, S. and Jentsch, S. (2009). "Principles of ubiquitin and SUMO modifications in DNA repair." Nature 458(7237): 461-7.

Bernier-Villamor, V., Sampson, D. A., Matunis, M. J. and Lima, C. D. (2002). "Structural basis for E2-mediated SUMO conjugation revealed by a complex between ubiquitin-conjugating enzyme Ubc9 and RanGAP1." Cell 108(3): 34556.

Besnault-Mascard, L., Leprince, C., Auffredou, M. T., Meunier, B., et al. (2005). "Caspase-8 sumoylation is associated with nuclear localization." Oncogene 24(20): 3268-73.

Birnboim, H. C. and Doly, J. (1979). "A rapid alkaline extraction procedure for screening recombinant plasmid DNA." Nucleic Acids Res 7(6): 1513-23.

Boggio, R. and Chiocca, S. (2005). "Gam1 and the SUMO pathway." Cell Cycle 4(4): 533-5.

Boggio, R., Passafaro, A. and Chiocca, S. (2007). "Targeting SUMO E1 to ubiquitin ligases: a viral strategy to counteract sumoylation." J Biol Chem 282(21): 15376-82.

Bohnsack, R. N. and Haas, A. L. (2003). "Conservation in the mechanism of Nedd8 activation by the human AppBp1-Uba3 heterodimer." J Biol Chem 278(29): 26823-30.

Bonifacino, Dasso, Harford, Lippincott-Schwartz, et al. (2000). Current Protocols in Cell Biology, John Whiley \& Sons.

Bossis, G., Chmielarska, K., Gartner, U., Pichler, A., et al. (2005). "A fluorescence resonance energy transfer-based assay to study SUMO modification in solution." Methods Enzymol 398: 20-32.

Bossis, G. and Melchior, F. (2006). "Regulation of SUMOylation by reversible oxidation of SUMO conjugating enzymes." Mol Cell 21(3): 349-57. 
Bossis, G. and Melchior, F. (2006). "SUMO: regulating the regulator." Cell Div 1: 13.

Brownawell, A. M. and Macara, I. G. (2002). "Exportin-5, a novel karyopherin, mediates nuclear export of double-stranded RNA binding proteins." 156(1): 53-64.

Chaikam, V. and Karlson, D. T. "Response and transcriptional regulation of rice SUMOylation system during development and stress conditions." BMB Rep 43(2): 103-9.

Chelsky, D., Ralph, R. and Jonak, G. (1989). "Sequence requirements for synthetic peptide-mediated translocation to the nucleus." Mol Cell Biol 9(6): 2487-92.

Cheng, C. H., Lo, Y. H., Liang, S. S., Ti, S. C., et al. (2006). "SUMO modifications control assembly of synaptonemal complex and polycomplex in meiosis of Saccharomyces cerevisiae." Genes Dev 20(15): 2067-81.

Cheng, T. S., Chang, L. K., Howng, S. L., Lu, P. J., et al. (2006). "SUMO-1 modification of centrosomal protein hNinein promotes $\mathrm{hNinein}$ nuclear localization." Life Sci 78(10): 1114-20.

Chi, N. C., Adam, E. J. and Adam, S. A. (1995). "Sequence and characterization of cytoplasmic nuclear protein import factor p97." J Cell Biol 130(2): 265-74.

Chi, N. C. and Adam, S. A. (1997). "Functional domains in nuclear import factor p97 for binding the nuclear localization sequence receptor and the nuclear pore." Mol Biol Cell 8(6): 945-56.

Coligan, Dunn, Ploegh, Speicher, et al. (2003). Current Protocols in Protein Science, John Whiley \& Sons.

Conti, E. and Kuriyan, J. (2000). "Crystallographic analysis of the specific yet versatile recognition of distinct nuclear localization signals by karyopherin alpha." Structure 8(3): 329-38.

Coussen, F. and Choquet, D. (2007). "Neuroscience: wrestling with SUMO." Nature 447(7142): 271-2.

Cronshaw, J. M., Krutchinsky, A. N., Zhang, W., Chait, B. T., et al. (2002). "Proteomic analysis of the mammalian nuclear pore complex." J Cell Biol 158(5): 915-27.

Dadke, S., Cotteret, S., Yip, S. C., Jaffer, Z. M., et al. (2007). "Regulation of protein tyrosine phosphatase 1B by sumoylation." Nat Cell Biol 9(1): 80-5.

Dang, C. V. and Lee, W. M. (1988). "Identification of the human c-myc protein nuclear translocation signal." Mol Cell Biol 8(10): 4048-54. 
del Olmo, M., Mizrahi, N., Gross, S. and Moore, C. L. (1997). "The Uba2 and Ufd1 proteins of Saccharomyces cerevisiae interact with poly $(A)$ polymerase and affect the polyadenylation activity of cell extracts." Mol Gen Genet 255(2): 20918.

Desterro, J. M., Rodriguez, M. S. and Hay, R. T. (1998). "SUMO-1 modification of IkappaBalpha inhibits NF-kappaB activation." Mol Cell 2(2): 233-9.

Desterro, J. M., Rodriguez, M. S., Kemp, G. D. and Hay, R. T. (1999). "Identification of the enzyme required for activation of the small ubiquitin-like protein SUMO1." J Biol Chem 274(15): 10618-24.

Desterro, J. M., Thomson, J. and Hay, R. T. (1997). "Ubch9 conjugates SUMO but not ubiquitin." FEBS Lett 417(3): 297-300.

Di Bacco, A., Ouyang, J., Lee, H. Y., Catic, A., et al. (2006). "The SUMO-specific protease SENP5 is required for cell division." Mol Cell Biol 26(12): 4489-98.

Dingwall, C., Sharnick, S. V. and Laskey, R. A. (1982). "A polypeptide domain that specifies migration of nucleoplasmin into the nucleus." Cell 30(2): 449-58.

Dohmen, R. J., Stappen, R., McGrath, J. P., Forrova, H., et al. (1995). "An essential yeast gene encoding a homolog of ubiquitin-activating enzyme." J Biol Chem 270(30): 18099-109.

Donaghue, C., Bates, H. and Cotterill, S. (2001). "Identification and characterisation of the Drosophila homologue of the yeast Uba2 gene." Biochim Biophys Acta 1518(1-2): 210-4.

Duncan, L. M., Piper, S., Dodd, R. B., Saville, M. K., et al. (2006). "Lysine-63-linked ubiquitination is required for endolysosomal degradation of class I molecules." EMBO J 25(8): 1635-45.

Engelsma, D., Bernad, R., Calafat, J. and Fornerod, M. (2004). "Supraphysiological nuclear export signals bind CRM1 independently of RanGTP and arrest at Nup358." EMBO J 23(18): 3643-52.

Fabbro, M. and Henderson, B. R. (2003). "Regulation of tumor suppressors by nuclear-cytoplasmic shuttling." Exp Cell Res 282(2): 59-69.

Finley, D. (2009). "Recognition and processing of ubiquitin-protein conjugates by the proteasome." Annu Rev Biochem 78: 477-513.

Fischer, U., Huber, J., Boelens, W. C., Mattaj, I. W., et al. (1995). "The HIV-1 Rev activation domain is a nuclear export signal that accesses an export pathway used by specific cellular RNAs." Cell 82(3): 475-83. 
Fitzgerald, M. E. and Drohat, A. C. (2008). "Coordinating the initial steps of base excision repair. Apurinic/apyrimidinic endonuclease 1 actively stimulates thymine DNA glycosylase by disrupting the product complex." J Biol Chem 283(47): 32680-90.

Fornerod, M., Ohno, M., Yoshida, M. and Mattaj, I. W. (1997). "CRM1 is an export receptor for leucine-rich nuclear export signals." Cell 90(6): 1051-60.

Fraser, A. G., Kamath, R. S., Zipperlen, P., Martinez-Campos, M., et al. (2000). "Functional genomic analysis of C. elegans chromosome I by systematic RNA interference." Nature 408(6810): 325-30.

Fried, H. and Kutay, U. (2003). "Nucleocytoplasmic transport: taking an inventory." Cell Mol Life Sci 60(8): 1659-88.

Gallouzi, I. E. and Steitz, J. A. (2001). "Delineation of mRNA export pathways by the use of cell-permeable peptides." Science 294(5548): 1895-901.

Garcia-Dominguez, M. and Reyes, J. C. (2009). "SUMO association with repressor complexes, emerging routes for transcriptional control." Biochim Biophys Acta 1789(6-8): 451-9.

Geetha, T., Jiang, J. and Wooten, M. W. (2005). "Lysine 63 polyubiquitination of the nerve growth factor receptor TrkA directs internalization and signaling." Mol Cell 20(2): 301-12.

Geiss-Friedlander, R. and Melchior, F. (2007). "Concepts in sumoylation: a decade on." Nat Rev Mol Cell Biol 8(12): 947-56.

Gill, G. (2005). "Something about SUMO inhibits transcription." Curr Opin Genet Dev 15(5): 536-41.

Giorgino, F., de Robertis, O., Laviola, L., Montrone, C., et al. (2000). "The sentrinconjugating enzyme mUbc9 interacts with GLUT4 and GLUT1 glucose transporters and regulates transporter levels in skeletal muscle cells." Proc Natl Acad Sci U S A 97(3): 1125-30.

Golebiowski, F., Matic, I., Tatham, M. H., Cole, C., et al. (2009). "System-wide changes to SUMO modifications in response to heat shock." Sci Signal 2(72): ra24.

Gong, L., Li, B., Millas, S. and Yeh, E. T. (1999). "Molecular cloning and characterization of human AOS1 and UBA2, components of the sentrinactivating enzyme complex." FEBS Lett 448(1): 185-9. 
Gong, L., Millas, S., Maul, G. G. and Yeh, E. T. (2000). "Differential regulation of

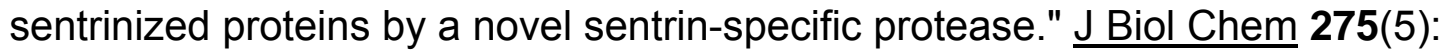
3355-9.

Gong, L. and Yeh, E. T. (2006). "Characterization of a family of nucleolar SUMOspecific proteases with preference for SUMO-2 or SUMO-3." $\mathrm{J}$ Biol Chem 281(23): 15869-77.

Gorlich, D., Pante, N., Kutay, U., Aebi, U., et al. (1996). "Identification of different roles for RanGDP and RanGTP in nuclear protein import." EMBO J 15(20): 5584-94.

Guo, D., Li, M., Zhang, Y., Yang, P., et al. (2004). "A functional variant of SUMO4, a new I kappa B alpha modifier, is associated with type 1 diabetes." Nat Genet 36(8): 837-41.

Haas, A. L. and Rose, I. A. (1982). "The mechanism of ubiquitin activating enzyme. A kinetic and equilibrium analysis." J Biol Chem 257(17): 10329-37.

Haas, A. L., Warms, J. V., Hershko, A. and Rose, I. A. (1982). "Ubiquitin-activating enzyme. Mechanism and role in protein-ubiquitin conjugation." $\underline{\mathrm{J} \text { Biol Chem }}$ 257(5): 2543-8.

Haglund, K., Di Fiore, P. P. and Dikic, I. (2003). "Distinct monoubiquitin signals in receptor endocytosis." Trends Biochem Sci 28(11): 598-603.

Haglund, K., Sigismund, S., Polo, S., Szymkiewicz, I., et al. (2003). "Multiple monoubiquitination of RTKs is sufficient for their endocytosis and degradation." Nat Cell Biol 5(5): 461-6.

Hang, J. and Dasso, M. (2002). "Association of the human SUMO-1 protease SENP2 with the nuclear pore." J Biol Chem 277(22): 19961-6.

Hardeland, U., Steinacher, R., Jiricny, J. and Schar, P. (2002). "Modification of the human thymine-DNA glycosylase by ubiquitin-like proteins facilitates enzymatic turnover." EMBO J 21(6): 1456-64.

Harreman, M. T., Kline, T. M., Milford, H. G., Harben, M. B., et al. (2004). "Regulation of nuclear import by phosphorylation adjacent to nuclear localization signals." J Biol Chem 279(20): 20613-21.

Hatfield, P. M. and Vierstra, R. D. (1992). "Multiple forms of ubiquitin-activating enzyme E1 from wheat. Identification of an essential cysteine by in vitro mutagenesis." J Biol Chem 267(21): 14799-803.

Hay, R. T. (2005). "SUMO: a history of modification." Mol Cell 18(1): 1-12. 
Hayashi, N., Shirakura, H., Uehara, T. and Nomura, Y. (2006). "Relationship between SUMO-1 modification of caspase-7 and its nuclear localization in human neuronal cells." Neurosci Lett 397(1-2): 5-9.

Hershko, A. and Ciechanover, A. (1998). "The ubiquitin system." Annu Rev Biochem 67: 425-79.

Hicke, L. and Dunn, R. (2003). "Regulation of membrane protein transport by ubiquitin and ubiquitin-binding proteins." Annu Rev Cell Dev Biol 19: 141-72.

Hochstrasser, M. (2000). "Evolution and function of ubiquitin-like protein-conjugation systems." Nat Cell Biol 2(8): E153-7.

Hochstrasser, M. (2001). "SP-RING for SUMO: new functions bloom for a ubiquitinlike protein." Cell 107(1): 5-8.

Hoege, C., Pfander, B., Moldovan, G. L., Pyrowolakis, G., et al. (2002). "RAD6dependent DNA repair is linked to modification of PCNA by ubiquitin and SUMO." Nature 419(6903): 135-41.

Hood, J. K. and Silver, P. A. (1998). "Cse1p is required for export of Srp1p/importinalpha from the nucleus in Saccharomyces cerevisiae." J Biol Chem 273(52): 35142-6.

Hsiao, H. H., Meulmeester, E., Frank, B. T., Melchior, F., et al. (2009). "'ChopNSpice," a mass spectrometric approach that allows identification of endogenous small ubiquitin-like modifier-conjugated peptides." Mol Cell Proteomics 8(12): 2664-75.

Hu, T., Guan, T. and Gerace, L. (1996). "Molecular and functional characterization of the p62 complex, an assembly of nuclear pore complex glycoproteins." $\underline{\mathrm{J} \text { Cell }}$ Biol 134(3): 589-601.

Huang, D. T., Hunt, H. W., Zhuang, M., Ohi, M. D., et al. (2007). "Basis for a ubiquitin-like protein thioester switch toggling E1-E2 affinity." Nature 445(7126): 394-8.

Hutten, S. and Kehlenbach, R. H. (2006). "Nup214 is required for CRM1-dependent nuclear protein export in vivo." Mol Cell Biol 26(18): 6772-85.

Hutten, S. and Kehlenbach, R. H. (2007). "CRM1-mediated nuclear export: to the pore and beyond." Trends Cell Biol 17(4): 193-201.

Ikeda, F. and Dikic, I. (2008). "Atypical ubiquitin chains: new molecular signals. 'Protein Modifications: Beyond the Usual Suspects' review series." EMBO Rep 9(6): 536-42. 
Jackson, P. K. (2001). "A new RING for SUMO: wrestling transcriptional responses into nuclear bodies with PIAS family E3 SUMO ligases." Genes Dev 15(23): 3053-8.

Jakel, S. and Gorlich, D. (1998). "Importin beta, transportin, RanBP5 and RanBP7 mediate nuclear import of ribosomal proteins in mammalian cells." EMBO J 17(15): 4491-502.

Janke, C., Magiera, M. M., Rathfelder, N., Taxis, C., et al. (2004). "A versatile toolbox for PCR-based tagging of yeast genes: new fluorescent proteins, more markers and promoter substitution cassettes." Yeast 21(11): 947-62.

Jin, L., Williamson, A., Banerjee, S., Philipp, I., et al. (2008). "Mechanism of ubiquitinchain formation by the human anaphase-promoting complex." Cell 133(4): 653-65.

Johnson, E. S. (2004). "Protein modification by SUMO." Annu Rev Biochem 73: 35582.

Johnson, E. S. and Blobel, G. (1997). "Ubc9p is the conjugating enzyme for the ubiquitin-like protein Smt3p." J Biol Chem 272(43): 26799-802.

Johnson, E. S. and Gupta, A. A. (2001). "An E3-like factor that promotes SUMO conjugation to the yeast septins." Cell 106(6): 735-44.

Johnson, E. S., Schwienhorst, I., Dohmen, R. J. and Blobel, G. (1997). "The ubiquitin-like protein Smt3p is activated for conjugation to other proteins by an Aos1p/Uba2p heterodimer." EMBO J 16(18): 5509-19.

Jones, D. H. and Matus, A. I. (1974). "Isolation of synaptic plasma membrane from brain by combined flotation-sedimentation density gradient centrifugation." Biochim Biophys Acta 356(3): 276-87.

Junn, E., Taniguchi, H., Jeong, B. S., Zhao, X., et al. (2005). "Interaction of DJ-1 with Daxx inhibits apoptosis signal-regulating kinase 1 activity and cell death." Proc Natl Acad Sci U S A 102(27): 9691-6.

Kadare, G., Toutant, M., Formstecher, E., Corvol, J. C., et al. (2003). "PIAS1mediated sumoylation of focal adhesion kinase activates its autophosphorylation." J Biol Chem 278(48): 47434-40.

Kaffman, A. and O'Shea, E. K. (1999). "Regulation of nuclear localization: a key to a door." Annu Rev Cell Dev Biol 15: 291-339.

Kagey, M. H., Melhuish, T. A., Powers, S. E. and Wotton, D. (2005). "Multiple activities contribute to Pc2 E3 function." EMBO J 24(1): 108-19. 
Kagey, M. H., Melhuish, T. A. and Wotton, D. (2003). "The polycomb protein Pc2 is a SUMO E3." Cell 113(1): 127-37.

Kalderon, D., Richardson, W. D., Markham, A. F. and Smith, A. E. (1984). "Sequence requirements for nuclear location of simian virus 40 large-T antigen." Nature 311(5981): 33-8.

Kang, J. S., Saunier, E. F., Akhurst, R. J. and Derynck, R. (2008). "The type I TGFbeta receptor is covalently modified and regulated by sumoylation." Nat Cell Biol 10(6): 654-64.

Kataoka, N., Bachorik, J. L. and Dreyfuss, G. (1999). "Transportin-SR, a nuclear import receptor for SR proteins." J Cell Biol 145(6): 1145-52.

Kehlenbach, R. H. (2009). Nuclear transport. Austin, Tex., Landes Bioscience.

Kehlenbach, R. H., Assheuer, R., Kehlenbach, A., Becker, J., et al. (2001). "Stimulation of nuclear export and inhibition of nuclear import by a Ran mutant deficient in binding to Ran-binding protein 1." J Biol Chem 276(17): 14524-31.

Kehlenbach, R. H., Dickmanns, A., Kehlenbach, A., Guan, T., et al. (1999). "A role for RanBP1 in the release of CRM1 from the nuclear pore complex in a terminal step of nuclear export." J Cell Biol 145(4): 645-57.

Kerscher, O., Felberbaum, R. and Hochstrasser, M. (2006). "Modification of proteins by ubiquitin and ubiquitin-like proteins." Annu Rev Cell Dev Biol 22: 159-80.

Kim, H. T., Kim, K. P., Lledias, F., Kisselev, A. F., et al. (2007). "Certain pairs of ubiquitin-conjugating enzymes (E2s) and ubiquitin-protein ligases (E3s) synthesize nondegradable forked ubiquitin chains containing all possible isopeptide linkages." J Biol Chem 282(24): 17375-86.

Kim, H. T., Kim, K. P., Uchiki, T., Gygi, S. P., et al. (2009). "S5a promotes protein degradation by blocking synthesis of nondegradable forked ubiquitin chains." EMBO J 28(13): 1867-77.

Kim, K. I., Baek, S. H., Jeon, Y. J., Nishimori, S., et al. (2000). "A new SUMO-1specific protease, SUSP1, that is highly expressed in reproductive organs." $\underline{J}$ Biol Chem 275(19): 14102-6.

Kodiha, M., Tran, D., Morogan, A., Qian, C., et al. (2009). "Dissecting the signaling events that impact classical nuclear import and target nuclear transport factors." PLoS ONE 4(12): e8420.

Komander, D. (2009). "The emerging complexity of protein ubiquitination." Biochem Soc Trans 37(Pt 5): 937-53. 
Koster, M., Frahm, T. and Hauser, H. (2005). "Nucleocytoplasmic shuttling revealed by FRAP and FLIP technologies." Curr Opin Biotechnol 16(1): 28-34.

Kotaja, N., Karvonen, U., Janne, O. A. and Palvimo, J. J. (2002). "PIAS proteins modulate transcription factors by functioning as SUMO-1 ligases." Mol Cell Biol 22(14): 5222-34.

Kunzler, M. and Hurt, E. C. (1998). "Cse1p functions as the nuclear export receptor for importin alpha in yeast." FEBS Lett 433(3): 185-90.

Kurepa, J., Walker, J. M., Smalle, J., Gosink, M. M., et al. (2003). "The small ubiquitin-like modifier (SUMO) protein modification system in Arabidopsis. Accumulation of SUMO1 and -2 conjugates is increased by stress." $\underline{\mathrm{J} \text { Biol }}$ Chem 278(9): 6862-72.

Kutay, U., Bischoff, F. R., Kostka, S., Kraft, R., et al. (1997). "Export of importin alpha from the nucleus is mediated by a specific nuclear transport factor." Cell 90(6): 1061-71.

Kutay, U., Lipowsky, G., Izaurralde, E., Bischoff, F. R., et al. (1998). "Identification of a tRNA-specific nuclear export receptor." Mol Cell 1(3): 359-69.

Kyhse-Andersen, J. (1984). "Electroblotting of multiple gels: a simple apparatus without buffer tank for rapid transfer of proteins from polyacrylamide to nitrocellulose." J Biochem Biophys Methods 10(3-4): 203-9.

Laemmli, U. K. (1970). "Cleavage of structural proteins during the assembly of the head of bacteriophage T4." Nature 227(5259): 680-5.

Lai, M. C., Lin, R. I. and Tarn, W. Y. (2001). "Transportin-SR2 mediates nuclear import of phosphorylated SR proteins." Proc Natl Acad Sci U S A 98(18): 10154-9.

Lee, G. W., Melchior, F., Matunis, M. J., Mahajan, R., et al. (1998). "Modification of Ran GTPase-activating protein by the small ubiquitin-related modifier SUMO-1 requires Ubc9, an E2-type ubiquitin-conjugating enzyme homologue." J Biol Chem 273(11): 6503-7.

Lee, Y. J., Miyake, S., Wakita, H., McMullen, D. C., et al. (2007). "Protein SUMOylation is massively increased in hibernation torpor and is critical for the cytoprotection provided by ischemic preconditioning and hypothermia in SHSY5Y cells." J Cereb Blood Flow Metab 27(5): 950-62. 
Lehmann, A. R., Niimi, A., Ogi, T., Brown, S., et al. (2007). "Translesion synthesis: Yfamily polymerases and the polymerase switch." DNA Repair (Amst) 6(7): 8919.

Li, S. J. and Hochstrasser, M. (1999). "A new protease required for cell-cycle progression in yeast." Nature 398(6724): 246-51.

Li, S. J. and Hochstrasser, M. (2000). "The yeast ULP2 (SMT4) gene encodes a novel protease specific for the ubiquitin-like Smt3 protein." Mol Cell Biol 20(7): 2367-77.

Li, S. J. and Hochstrasser, M. (2003). "The Ulp1 SUMO isopeptidase: distinct domains required for viability, nuclear envelope localization, and substrate specificity." J Cell Biol 160(7): 1069-81.

Lipowsky, G., Bischoff, F. R., Schwarzmaier, P., Kraft, R., et al. (2000). "Exportin 4: a mediator of a novel nuclear export pathway in higher eukaryotes." EMBO J 19(16): 4362-71.

Liu, G. H. and Gerace, L. (2009). "Sumoylation regulates nuclear localization of lipin1alpha in neuronal cells." PLoS ONE 4(9): e7031.

Lois, L. M. and Lima, C. D. (2005). "Structures of the SUMO E1 provide mechanistic insights into SUMO activation and E2 recruitment to E1." EMBO J 24(3): 43951.

Long, X. and Griffith, L. C. (2000). "Identification and characterization of a SUMO-1 conjugation system that modifies neuronal calcium/calmodulin-dependent protein kinase II in Drosophila melanogaster." J Biol Chem 275(52): 40765-76.

Love, K. R., Catic, A., Schlieker, C. and Ploegh, H. L. (2007). "Mechanisms, biology and inhibitors of deubiquitinating enzymes." Nat Chem Biol 3(11): 697-705.

Lund, E., Guttinger, S., Calado, A., Dahlberg, J. E., et al. (2004). "Nuclear export of microRNA precursors." Science 303(5654): 95-8.

Lyst, M. J. and Stancheva, I. (2007). "A role for SUMO modification in transcriptional repression and activation." Biochem Soc Trans 35(Pt 6): 1389-92.

Mahajan, R., Delphin, C., Guan, T., Gerace, L., et al. (1997). "A small ubiquitinrelated polypeptide involved in targeting RanGAP1 to nuclear pore complex protein RanBP2." Cell 88(1): 97-107.

Makkerh, J. P., Dingwall, C. and Laskey, R. A. (1996). "Comparative mutagenesis of nuclear localization signals reveals the importance of neutral and acidic amino acids." Curr Biol 6(8): 1025-7. 
Maniatis, Fritsch and Sambrook (1989). Molecular Cloning: A Laboratory Manual, Cold Spring Harbor Laboratory Press.

Mao, Y., Sun, M., Desai, S. D. and Liu, L. F. (2000). "SUMO-1 conjugation to topoisomerase I: A possible repair response to topoisomerase-mediated DNA damage." Proc Natl Acad Sci U S A 97(8): 4046-51.

Martin, S., Nishimune, A., Mellor, J. R. and Henley, J. M. (2007). "SUMOylation regulates kainate-receptor-mediated synaptic transmission." Nature 447(7142): 321-5.

Martin, S., Wilkinson, K. A., Nishimune, A. and Henley, J. M. (2007). "Emerging extranuclear roles of protein SUMOylation in neuronal function and dysfunction." Nat Rev Neurosci 8(12): 948-59.

Matic, I., van Hagen, M., Schimmel, J., Macek, B., et al. (2008). "In vivo identification of human small ubiquitin-like modifier polymerization sites by high accuracy mass spectrometry and an in vitro to in vivo strategy." Mol Cell Proteomics 7(1): 132-44.

Matunis, M. J., Coutavas, E. and Blobel, G. (1996). "A novel ubiquitin-like modification modulates the partitioning of the Ran-GTPase-activating protein RanGAP1 between the cytosol and the nuclear pore complex." $\underline{\mathrm{J} \text { Cell Biol }}$ 135(6 Pt 1): 1457-70.

Melchior, F. (2000). "SUMO--nonclassical ubiquitin." Annu Rev Cell Dev Biol 16: 591626.

Merril, C. R., Goldman, D., Sedman, S. A. and Ebert, M. H. (1981). "Ultrasensitive stain for proteins in polyacrylamide gels shows regional variation in cerebrospinal fluid proteins." Science 211(4489): 1437-8.

Merrill, J. C., Melhuish, T. A., Kagey, M. H., Yang, S. H., et al. "A role for noncovalent SUMO interaction motifs in Pc2/CBX4 E3 activity." PLoS ONE 5(1): e8794.

Meulmeester, E., Kunze, M., Hsiao, H. H., Urlaub, H., et al. (2008). "Mechanism and consequences for paralog-specific sumoylation of ubiquitin-specific protease 25." Mol Cell 30(5): 610-9.

Mingot, J. M., Kostka, S., Kraft, R., Hartmann, E., et al. (2001). "Importin 13: a novel mediator of nuclear import and export." EMBO J 20(14): 3685-94. 
Miyauchi, Y., Yogosawa, S., Honda, R., Nishida, T., et al. (2002). "Sumoylation of Mdm2 by protein inhibitor of activated STAT (PIAS) and RanBP2 enzymes." $\underline{\mathrm{J}}$ Biol Chem 277(51): 50131-6.

Mo, Y. Y., Yu, Y., Shen, Z. and Beck, W. T. (2002). "Nucleolar delocalization of human topoisomerase I in response to topotecan correlates with sumoylation of the protein." J Biol Chem 277(4): 2958-64.

Moldovan, G. L., Pfander, B. and Jentsch, S. (2007). "PCNA, the maestro of the replication fork." Cell 129(4): 665-79.

Mosammaparast, N., Ewart, C. S. and Pemberton, L. F. (2002). "A role for nucleosome assembly protein 1 in the nuclear transport of histones $\mathrm{H} 2 \mathrm{~A}$ and H2B." EMBO J 21(23): 6527-38.

Mosammaparast, N., Jackson, K. R., Guo, Y., Brame, C. J., et al. (2001). "Nuclear import of histone $\mathrm{H} 2 \mathrm{~A}$ and $\mathrm{H} 2 \mathrm{~B}$ is mediated by a network of karyopherins." $\underline{\mathrm{J}}$ Cell Biol 153(2): 251-62.

Mosesson, Y. and Yarden, Y. (2006). "Monoubiquitylation: a recurrent theme in membrane protein transport." Isr Med Assoc J 8(4): 233-7.

Muhlhausser, P., Muller, E. C., Otto, A. and Kutay, U. (2001). "Multiple pathways contribute to nuclear import of core histones." EMBO Rep 2(8): 690-6.

Mukhopadhyay, D., Ayaydin, F., Kolli, N., Tan, S. H., et al. (2006). "SUSP1 antagonizes formation of highly SUMO2/3-conjugated species." $\underline{\mathrm{J} \text { Cell Biol }}$ 174(7): 939-49.

Mukhopadhyay, D. and Dasso, M. (2007). "Modification in reverse: the SUMO proteases." Trends Biochem Sci 32(6): 286-95.

Mullis, K. B. (1990). "The unusual origin of the polymerase chain reaction." Sci Am 262(4): 56-61, 64-5.

Nacerddine, K., Lehembre, F., Bhaumik, M., Artus, J., et al. (2005). "The SUMO pathway is essential for nuclear integrity and chromosome segregation in mice." Dev Cell 9(6): 769-79.

Nakielny, S., Siomi, M. C., Siomi, H., Michael, W. M., et al. (1996). "Transportin: nuclear transport receptor of a novel nuclear protein import pathway." Exp Cell Res 229(2): 261-6.

Nishida, T., Tanaka, H. and Yasuda, H. (2000). "A novel mammalian Smt3-specific isopeptidase 1 (SMT3IP1) localized in the nucleolus at interphase." Eur J Biochem 267(21): 6423-7. 
Okuma, T., Honda, R., Ichikawa, G., Tsumagari, N., et al. (1999). "In vitro SUMO-1 modification requires two enzymatic steps, E1 and E2." Biochem Biophys Res Commun 254(3): 693-8.

Olsen, S. K., Capili, A. D., Lu, X., Tan, D. S., et al. "Active site remodelling accompanies thioester bond formation in the SUMO E1." Nature 463(7283): 906-12.

Ouyang, J. and Gill, G. (2009). "SUMO engages multiple corepressors to regulate chromatin structure and transcription." Epigenetics 4(7): 440-4.

Ouyang, J., Valin, A. and Gill, G. (2009). "Regulation of transcription factor activity by SUMO modification." Methods Mol Biol 497: 141-52.

Owerbach, D., McKay, E. M., Yeh, E. T., Gabbay, K. H., et al. (2005). "A proline-90 residue unique to SUMO-4 prevents maturation and sumoylation." Biochem Biophys Res Commun 337(2): 517-20.

Panier, S. and Durocher, D. (2009). "Regulatory ubiquitylation in response to DNA double-strand breaks." DNA Repair (Amst) 8(4): 436-43.

Papouli, E., Chen, S., Davies, A. A., Huttner, D., et al. (2005). "Crosstalk between SUMO and ubiquitin on PCNA is mediated by recruitment of the helicase Srs2p." Mol Cell 19(1): 123-33.

Paraskeva, E., Izaurralde, E., Bischoff, F. R., Huber, J., et al. (1999). "CRM1mediated recycling of snurportin 1 to the cytoplasm." J Cell Biol 145(2): 25564.

Park, J., Seo, T., Kim, H. and Choe, J. (2005). "Sumoylation of the novel protein $\mathrm{hRIP}\{$ beta $\}$ is involved in replication protein A deposition in PML nuclear bodies." Mol Cell Biol 25(18): 8202-14.

Pfander, B., Moldovan, G. L., Sacher, M., Hoege, C., et al. (2005). "SUMO-modified PCNA recruits Srs2 to prevent recombination during $S$ phase." Nature 436(7049): 428-33.

Pichler, A., Gast, A., Seeler, J. S., Dejean, A., et al. (2002). "The nucleoporin RanBP2 has SUMO1 E3 ligase activity." Cell 108(1): 109-20.

Pichler, A., Knipscheer, P., Oberhofer, E., van Dijk, W. J., et al. (2005). "SUMO modification of the ubiquitin-conjugating enzyme E2-25K." Nat Struct Mol Biol 12(3): 264-9. 
Pichler, A., Knipscheer, P., Saitoh, H., Sixma, T. K., et al. (2004). "The RanBP2 SUMO E3 ligase is neither HECT- nor RING-type." Nat Struct Mol Biol 11(10): 984-91.

Pichler, A. and Melchior, F. (2002). "Ubiquitin-related modifier SUMO1 and nucleocytoplasmic transport." Traffic 3(6): 381-7.

Pickart, C. M. (2001). "Mechanisms underlying ubiquitination." Annu Rev Biochem 70: 503-33.

Plafker, S. M., Plafker, K. S., Weissman, A. M. and Macara, I. G. (2004). "Ubiquitin charging of human class III ubiquitin-conjugating enzymes triggers their nuclear import." J Cell Biol 167(4): 649-59.

Pollard, V. W., Michael, W. M., Nakielny, S., Siomi, M. C., et al. (1996). "A novel receptor-mediated nuclear protein import pathway." Cell 86(6): 985-94.

Potts, P. R. and Yu, H. (2005). "Human MMS21/NSE2 is a SUMO ligase required for DNA repair." Mol Cell Biol 25(16): 7021-32.

Reverter, D. and Lima, C. D. (2005). "Insights into E3 ligase activity revealed by a SUMO-RanGAP1-Ubc9-Nup358 complex." Nature 435(7042): 687-92.

Rexach, M. and Blobel, G. (1995). "Protein import into nuclei: association and dissociation reactions involving transport substrate, transport factors, and nucleoporins." Cell 83(5): 683-92.

Ribbeck, K., Lipowsky, G., Kent, H. M., Stewart, M., et al. (1998). "NTF2 mediates nuclear import of Ran." EMBO J 17(22): 6587-98.

Robbins, J., Dilworth, S. M., Laskey, R. A. and Dingwall, C. (1991). "Two interdependent basic domains in nucleoplasmin nuclear targeting sequence: identification of a class of bipartite nuclear targeting sequence." Cell 64(3): 615-23.

Robert, T., Dervins, D., Fabre, F. and Gangloff, S. (2006). "Mrc1 and Srs2 are major actors in the regulation of spontaneous crossover." EMBO J 25(12): 2837-46.

Rosas-Acosta, G. and Wilson, V. G. (2008). "Identification of a nuclear export signal sequence for bovine papillomavirus E1 protein." Virology 373(1): 149-62.

Roscic, A., Moller, A., Calzado, M. A., Renner, F., et al. (2006). "Phosphorylationdependent control of Pc2 SUMO E3 ligase activity by its substrate protein HIPK2." Mol Cell 24(1): 77-89.

Roth, J., Dobbelstein, M., Freedman, D. A., Shenk, T., et al. (1998). "Nucleocytoplasmic shuttling of the hdm2 oncoprotein regulates the levels of the p53 
protein via a pathway used by the human immunodeficiency virus rev protein." EMBO J 17(2): 554-64.

Roukens, M. G., Alloul-Ramdhani, M., Vertegaal, A. C., Anvarian, Z., et al. (2008). "Identification of a new site of sumoylation on Tel (ETV6) uncovers a PIASdependent mode of regulating Tel function." Mol Cell Biol 28(7): 2342-57.

Sachdev, S., Bruhn, L., Sieber, H., Pichler, A., et al. (2001). "PIASy, a nuclear matrixassociated SUMO E3 ligase, represses LEF1 activity by sequestration into nuclear bodies." Genes Dev 15(23): 3088-103.

Sacher, M., Pfander, B., Hoege, C. and Jentsch, S. (2006). "Control of Rad52 recombination activity by double-strand break-induced SUMO modification." Nat Cell Biol 8(11): 1284-90.

Saitoh, H. and Hinchey, J. (2000). "Functional heterogeneity of small ubiquitin-related protein modifiers SUMO-1 versus SUMO-2/3." J Biol Chem 275(9): 6252-8.

Saitoh, H., Pu, R. T. and Dasso, M. (1997). "SUMO-1: wrestling with a new ubiquitinrelated modifier." Trends Biochem Sci 22(10): 374-6.

Sampson, D. A., Wang, M. and Matunis, M. J. (2001). "The small ubiquitin-like modifier-1 (SUMO-1) consensus sequence mediates Ubc9 binding and is essential for SUMO-1 modification." J Biol Chem 276(24): 21664-9.

Sanger, F., Nicklen, S. and Coulson, A. R. (1977). "DNA sequencing with chainterminating inhibitors." Proc Natl Acad Sci U S A 74(12): 5463-7.

Saracco, S. A., Miller, M. J., Kurepa, J. and Vierstra, R. D. (2007). "Genetic analysis of SUMOylation in Arabidopsis: conjugation of SUMO1 and SUMO2 to nuclear proteins is essential." Plant Physiol 145(1): 119-34.

Schiestl, R. H. and Gietz, R. D. (1989). "High efficiency transformation of intact yeast cells using single stranded nucleic acids as a carrier." Curr Genet 16(5-6): 339-46.

Schulman, B. A. and Harper, J. W. (2009). "Ubiquitin-like protein activation by E1 enzymes: the apex for downstream signalling pathways." Nat Rev Mol Cell Biol 10(5): 319-31.

Seeler, J. S. and Dejean, A. (2001). "SUMO: of branched proteins and nuclear bodies." Oncogene 20(49): 7243-9.

Seufert, W., Futcher, B. and Jentsch, S. (1995). "Role of a ubiquitin-conjugating enzyme in degradation of S- and M-phase cyclins." Nature 373(6509): 78-81. 
Shamsher, M. K., Ploski, J. and Radu, A. (2002). "Karyopherin beta 2B participates in mRNA export from the nucleus." Proc Natl Acad Sci U S A 99(22): 14195-9.

Sheeler, P. (1981). Centrifugation in Biology and Medical Science.

Shih, H. P., Hales, K. G., Pringle, J. R. and Peifer, M. (2002). "Identification of septininteracting proteins and characterization of the Smt3/SUMO-conjugation system in Drosophila." J Cell Sci 115(Pt 6): 1259-71.

Shirakura, H., Hayashi, N., Ogino, S., Tsuruma, K., et al. (2005). "Caspase recruitment domain of procaspase-2 could be a target for SUMO-1 modification through Ubc9." Biochem Biophys Res Commun 331(4): 1007-15.

Siomi, H. and Dreyfuss, G. (1995). "A nuclear localization domain in the hnRNP A1 protein." J Cell Biol 129(3): 551-60.

Siomi, M. C., Eder, P. S., Kataoka, N., Wan, L., et al. (1997). "Transportin-mediated nuclear import of heterogeneous nuclear RNP proteins." J Cell Biol 138(6): 1181-92.

Skaug, B., Jiang, X. and Chen, Z. J. (2009). "The role of ubiquitin in NF-kappaB regulatory pathways." Annu Rev Biochem 78: 769-96.

Smith, A., Brownawell, A. and Macara, I. G. (1998). "Nuclear import of Ran is mediated by the transport factor NTF2." Curr Biol 8(25): 1403-6.

Smith, P. K., Krohn, R. I., Hermanson, G. T., Mallia, A. K., et al. (1985). "Measurement of protein using bicinchoninic acid." Anal Biochem 150(1): 7685.

Sobko, A., Ma, H. and Firtel, R. A. (2002). "Regulated SUMOylation and ubiquitination of DdMEK1 is required for proper chemotaxis." Dev Cell 2(6): 745-56.

Spector, Goldman and Leinwand (1998). Cells: A Laboratory Manual, Cold Spring Harbor Laboratory Press.

Stade, K., Ford, C. S., Guthrie, C. and Weis, K. (1997). "Exportin 1 (Crm1p) is an essential nuclear export factor." Cell 90(6): 1041-50.

Stade, K., Vogel, F., Schwienhorst, I., Meusser, B., et al. (2002). "A lack of SUMO conjugation affects cNLS-dependent nuclear protein import in yeast." $\underline{\mathrm{J} \text { Biol }}$ Chem 277(51): 49554-61.

Stankovic-Valentin, N., Kozaczkiewicz, L., Curth, K. and Melchior, F. (2009). "An in vitro FRET-based assay for the analysis of SUMO conjugation and isopeptidase cleavage." Methods Mol Biol 497: 241-51. 
Steinacher, R. and Schar, P. (2005). "Functionality of human thymine DNA glycosylase requires SUMO-regulated changes in protein conformation." $\underline{\text { Curr }}$ Biol 15(7): 616-23.

Stelter, P. and Ulrich, H. D. (2003). "Control of spontaneous and damage-induced mutagenesis by SUMO and ubiquitin conjugation." Nature 425(6954): 188-91.

Stielow, B., Sapetschnig, A., Wink, C., Kruger, I., et al. (2008). "SUMO-modified Sp3 represses transcription by provoking local heterochromatic gene silencing." EMBO Rep 9(9): 899-906.

Stochaj, U., Rassadi, R. and Chiu, J. (2000). "Stress-mediated inhibition of the classical nuclear protein import pathway and nuclear accumulation of the small GTPase Gsp1p." FASEB J 14(14): 2130-2.

Stone, J., de Lange, T., Ramsay, G., Jakobovits, E., et al. (1987). "Definition of regions in human c-myc that are involved in transformation and nuclear localization." Mol Cell Biol 7(5): 1697-709.

Stuven, T., Hartmann, E. and Gorlich, D. (2003). "Exportin 6: a novel nuclear export receptor that is specific for profilin.actin complexes." EMBO J 22(21): 5928-40.

Takahashi, Y., Iwase, M., Strunnikov, A. V. and Kikuchi, Y. (2008). "Cytoplasmic sumoylation by PIAS-type Siz1-SUMO ligase." Cell Cycle 7(12): 1738-44.

Takahashi, Y. and Kikuchi, Y. (2005). "Yeast PIAS-type Ull1/Siz1 is composed of SUMO ligase and regulatory domains." J Biol Chem 280(43): 35822-8.

Takahashi, Y., Toh-e, A. and Kikuchi, Y. (2001). "A novel factor required for the SUMO1/Smt3 conjugation of yeast septins." Gene 275(2): 223-31.

Tang, Z., El Far, O., Betz, H. and Scheschonka, A. (2005). "Pias1 interaction and

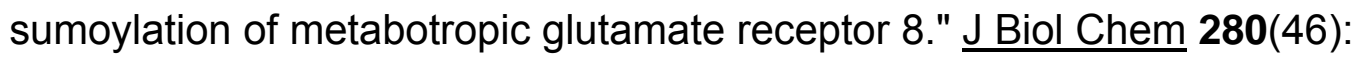
38153-9.

Tatham, M. H., Jaffray, E., Vaughan, O. A., Desterro, J. M., et al. (2001). "Polymeric chains of SUMO-2 and SUMO-3 are conjugated to protein substrates by SAE1/SAE2 and Ubc9." J Biol Chem 276(38): 35368-74.

Tatham, M. H., Kim, S., Jaffray, E., Song, J., et al. (2005). "Unique binding interactions among Ubc9, SUMO and RanBP2 reveal a mechanism for SUMO paralog selection." Nat Struct Mol Biol 12(1): 67-74.

Tempe, D., Piechaczyk, M. and Bossis, G. (2008). "SUMO under stress." Biochem Soc Trans 36(Pt 5): 874-8. 
Terry, L. J., Shows, E. B. and Wente, S. R. (2007). "Crossing the nuclear envelope: hierarchical regulation of nucleocytoplasmic transport." Science 318(5855): 1412-6.

Thrower, J. S., Hoffman, L., Rechsteiner, M. and Pickart, C. M. (2000). "Recognition of the polyubiquitin proteolytic signal." EMBO J 19(1): 94-102.

Ulrich, H. D. (2008). "The fast-growing business of SUMO chains." Mol Cell 32(3): 301-5.

Ulrich, H. D. (2009). "Regulating post-translational modifications of the eukaryotic replication clamp PCNA." DNA Repair (Amst) 8(4): 461-9.

Vertegaal, A. C. "SUMO chains: polymeric signals." Biochem Soc Trans 38(Pt 1): 469.

Vertegaal, A. C., Andersen, J. S., Ogg, S. C., Hay, R. T., et al. (2006). "Distinct and overlapping sets of SUMO-1 and SUMO-2 target proteins revealed by quantitative proteomics." Mol Cell Proteomics 5(12): 2298-310.

Vijay-Kumar, S., Bugg, C. E. and Cook, W. J. (1987). "Structure of ubiquitin refined at 1.8 A resolution." J Mol Biol 194(3): 531-44.

Walden, H., Podgorski, M. S., Huang, D. T., Miller, D. W., et al. (2003). "The structure of the APPBP1-UBA3-NEDD8-ATP complex reveals the basis for selective ubiquitin-like protein activation by an E1." Mol Cell 12(6): 1427-37.

Walsh, C. T., Garneau-Tsodikova, S. and Gatto, G. J., Jr. (2005). "Protein posttranslational modifications: the chemistry of proteome diversifications." Angew Chem Int Ed Engl 44(45): 7342-72.

Wang, Q. E., Zhu, Q., Wani, G., El-Mahdy, M. A., et al. (2005). "DNA repair factor XPC is modified by SUMO-1 and ubiquitin following UV irradiation." Nucleic Acids Res 33(13): 4023-34.

Watts, F. Z. (2006). "Sumoylation of PCNA: Wrestling with recombination at stalled replication forks." DNA Repair (Amst) 5(3): 399-403.

Wei, F., Scholer, H. R. and Atchison, M. L. (2007). "Sumoylation of Oct4 enhances its stability, DNA binding, and transactivation." J Biol Chem 282(29): 21551-60.

Wei, W., Yang, P., Pang, J., Zhang, S., et al. (2008). "A stress-dependent SUMO4 sumoylation of its substrate proteins." Biochem Biophys Res Commun 375(3): 454-9.

Weis, K. (2003). "Regulating access to the genome: nucleocytoplasmic transport throughout the cell cycle." Cell 112(4): 441-51. 
Welchman, R. L., Gordon, C. and Mayer, R. J. (2005). "Ubiquitin and ubiquitin-like proteins as multifunctional signals." Nat Rev Mol Cell Biol 6(8): 599-609.

Wen, W., Taylor, S. S. and Meinkoth, J. L. (1995). "The expression and intracellular distribution of the heat-stable protein kinase inhibitor is cell cycle regulated." $\underline{J}$ Biol Chem 270(5): 2041-6.

Werner, A., Moutty, M. C., Moller, U. and Melchior, F. (2009). "Performing in vitro sumoylation reactions using recombinant enzymes." Methods Mol Biol 497: 187-99.

Whitby, F. G., Xia, G., Pickart, C. M. and Hill, C. P. (1998). "Crystal structure of the human ubiquitin-like protein NEDD8 and interactions with ubiquitin pathway enzymes." J Biol Chem 273(52): 34983-91.

Wierenga, R. K. and Hol, W. G. (1983). "Predicted nucleotide-binding properties of p21 protein and its cancer-associated variant." Nature 302(5911): 842-4.

Wilken, N., Senecal, J. L., Scheer, U. and Dabauvalle, M. C. (1995). "Localization of the Ran-GTP binding protein RanBP2 at the cytoplasmic side of the nuclear pore complex." Eur J Cell Biol 68(3): 211-9.

Wohlwend, D., Strasser, A., Dickmanns, A., Doenecke, D., et al. (2007). "Thermodynamic analysis of $\mathrm{H} 1$ nuclear import: receptor tuning of importinbeta/importin7." J Biol Chem 282(14): 10707-19.

Wu, J., Matunis, M. J., Kraemer, D., Blobel, G., et al. (1995). "Nup358, a cytoplasmically exposed nucleoporin with peptide repeats, Ran-GTP binding sites, zinc fingers, a cyclophilin A homologous domain, and a leucine-rich region." J Biol Chem 270(23): 14209-13.

Xu, P., Duong, D. M., Seyfried, N. T., Cheng, D., et al. (2009). "Quantitative proteomics reveals the function of unconventional ubiquitin chains in proteasomal degradation." Cell 137(1): 133-45.

Yokoyama, N., Hayashi, N., Seki, T., Pante, N., et al. (1995). "A giant nucleopore protein that binds Ran/TC4." Nature 376(6536): 184-8.

Yunus, A. A. and Lima, C. D. (2009). "Structure of the Siz/PIAS SUMO E3 ligase Siz1 and determinants required for SUMO modification of PCNA." Mol Cell 35(5): 669-82.

Zhang, H., Saitoh, H. and Matunis, M. J. (2002). "Enzymes of the SUMO modification pathway localize to filaments of the nuclear pore complex." $\underline{\text { Mol Cell Biol }}$ 22(18): 6498-508. 
Zhao, J. (2007). "Sumoylation regulates diverse biological processes." Cell Mol Life Sci 64(23): 3017-33.

Zhao, X. and Blobel, G. (2005). "A SUMO ligase is part of a nuclear multiprotein complex that affects DNA repair and chromosomal organization." Proc Natl Acad Sci U S A 102(13): 4777-82.

Zhou, W., Ryan, J. J. and Zhou, H. (2004). "Global analyses of sumoylated proteins in Saccharomyces cerevisiae. Induction of protein sumoylation by cellular stresses." J Biol Chem 279(31): 32262-8.

Zunino, R., Schauss, A., Rippstein, P., Andrade-Navarro, M., et al. (2007). "The SUMO protease SENP5 is required to maintain mitochondrial morphology and function." J Cell Sci 120(Pt 7): 1178-88. 


\section{SUPPLEMENTARY MATERIAL}

Supplemantary Figure 1: Sequence alignment of Aos1. Amino acid sequences of Aos 1 from $H$. sapiens, M. musculus, $D$. rerio, $X$. laevis, $D$. melanogaster and S. cerevisiae were aligned using ClustalW2. Conserved clusters of basic amino acids potentially participating in nuclear import are underlined and highlighted in bold.
H. sapiens
M. musculus
D. rerio
$X$. laevis
D. melanogaster
S. cerevisiae
H. sapiens
M. musculus
D. rerio
$X$. laevis
D. melanogaster
S. cerevisiae
H. sapiens
M. musculus
D. rerio
$X$. laevis
D. melanogaster
S. cerevisiae
H. sapiens
M. musculus
D. rerio
$X$. laevis
D. melanogaster
S. cerevisiae
H. sapiens
M. musculus
D. rerio
$X$. laevis
D. melanogaster
S. cerevisiae
H. sapiens
M. musculus
D. rerio
$X$. laevis
D. melanogaster
S. cerevisiae
H. sapiens
M. musculus
D. rerio
$X$. laevis
D. melanogaster
S. cerevisiae

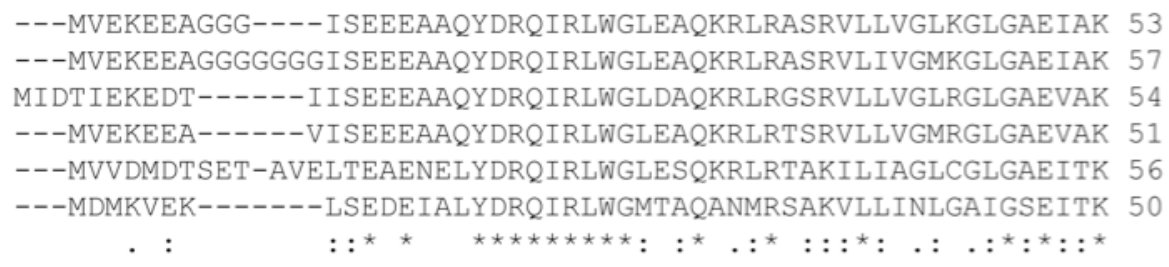
MIDTIEKEDT------IISEEEAAQYDRQIRLWGLDAQKRLRGSRVLLVGLRGLGAEVAK 54 ---MVEKEEA------VISEEEAAQYDRQIRLWGLEAQKRLRTSRVLLVGMRGLGAEVAK 51 ---MVVDMDTSET-AVELTEAENELYDRQIRLWGLESQKRLRTAKILIAGLCGLGAEITK 56 ---MDMKVEK-------LSEDEIALYDRQIRLWGMTAQANMRSAKVLLINLGAIGSEITK 50

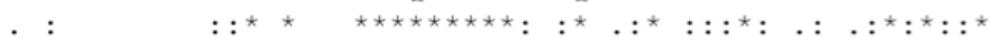

NLILAGVKGLTMLDHEQVTPEDPGAQFLIRTGSVGRNRAEASLERAQNLNPMVDVKVDTE 113 NLILAGVKGLTMLDHEQVSPEDPGAQFLIQTGSVGRNRAEASLERAQNLNPMVDVKVDTE 117 NLILAGVKGLTLLDHEQVTEESRRAQFLI PVDADGQNHAQASLERAQFLNPMVEVKADTE 114 NLILAGVKALTLLDHEQVSSEDSRAQFLIPSGSLGQNRAEASLNRARNLNPMVSVEADTE 111 NI I LSGVNSVKLLDDKDVTEEDFCSQFLVPRESLNTNRAEASLTRARALNPMVDISADRE 116 SIVLSGIGHLTILDGHMVTEEDLGSQFFIGSEDVGQWKIDATKERIQDLNPRVELNFDKQ 110 $.:{ }^{\star}:{ }^{\star}: \quad::^{\star \star} .{ }^{\star}:{ }^{\star} . \quad:^{\star \star}:: \quad$. $::^{\star}:{ }^{\star}:{ }^{\star \star}{ }^{\star} .:{ }^{\star}:$

DIEKKPESFFTQFDAVCLTCCSRDVIVKVDQICHKNSIKFFTGDVFGYHGYTFANLG-EH 172 DVEKKPESFFTKFDAVCLTCCSRDVIIKVDQICHRNSIKFFTGDVFGYHGYTFANLG-EH 176 PVESKPDDFFFQFDAVCLTRCSRDLMVRVDQLCASRNIKVFCGDVYGYNGYMFSDLGQEY 174 NINQKSDDFFTQFDVVCLTSCPSDLLVRVNHICHKHNIKFFTGDVYGYHGSMFADLG-EH 170 PLKEKTSEFFGQFDVVVVNGATNEELLRIDTICRDLGVKF IATDVWGTFGFYFASLQ-KH 175 DLQEKDEEFFQQEDLVVATEMQIDEAIKINTLTRKLNIPLYVAGSNGLFAYVEIDLI--- 167 $: .^{\star} \ldots{ }^{\star \star}:{ }^{\star \star} \star \quad . \quad:::: \quad: \quad .: . \quad .{ }^{\star} . .^{\star}$

EFVEEKTKVAKVSQGVEDGPDT KRAKLDSSE----------TTMVKKKVVFCPVKEALEV 222 EFVEEKTKVAKVSQGVEDGPEA KRAKLDSSE--------TTMV KKK VLFCPVKEALEV 226 HYVEEKPKVVKGSNEANDGPEAKKPKIDPNE---------TTMV KKTISFCSLKEALEV 224 EFVEEKAKVTKAKPLVEDGPEAKKAKIDPTE---------TILVKKKVQFCPLKDALEI 220 SYVEDVINHKVVAN-------SEKKKKYETV---------SIPTQRDVDYPGYSAWLDF 218 EFISEDEKLQSVRPTTVGPISSNRS I IEVTTRKDEEDEKKTYERIKTKNCYRPLNEVLST 227 $::: \quad::: \quad$ : $\quad: \quad:$. $^{\star}$.

DWSSEKAKAALKRTTSDYFLLQVLLKFRTDKGRDPSSDTYEEDSELLLQIRNDVLDSLGI 282 DWSGEKAKAALKRTAPDYFLLQVLLKFRTDKGRDPTSESYKEDAELLLQIRNDVFDSLGI 286 DWTTEKAKSSLKRIPADYFLLQVLLKFRTDKGRDPQPDSFAEDSQLLLQIRDDVLETMGL 284 DWRSEKAKSALKKTPTDYFLLQVLMKFRTDKGRDPQPSSYQEDSELLLQICSDVLDSLGV 280 DVTEPSYLRKLKRNGPGVLLLSVLQKFRTTHKRDPSYKTREADLELLRGIRDELLPNS-- 276 ATLKEKMTQRQLKR-VTS ILPLTLSILQYDLNQKGKAISFEQMKRDAAVWCENLGVPATV 286

SPDLLPEDFVRYCFSEMAPVCAVVGGILAQEIVKALSQRDPPHNNFFFFDGMKGNGIVEC 342 SPDLLPDDFVRYCFSEMAPVCAVVGGILAQEIVKALSQRDPPHNNFFFFDGMKGSGIVEC 346 SSDLLPNTFVSYCFSEMSPVCAVVGGVLGQEIVKALSQRDAPHRNFFFFDGLKGSGVVDY 344 SPDLLPKDFASYCFSEMAPVCAVVGGVLGQEIVKALSLRDAPHNNFFFFDGKTSNGIVDC 340 ---ILGDEALGLIFAQISPAVAVVGGVVAQEVIKVVTKLEAPHRNLFVFDPETCAGYVEA 333 VKDDYVQQFIKQKGIEFAPVAAI IGGAVAQDVINILGKRLSPLNNFIVFDGITLDMPLFE 346

$$
::{ }^{\star} .{ }^{\star}::{ }^{\star \star}:{ }^{\star}::::: \quad:{ }^{\star} \cdot{ }^{\star}:: .^{\star \star} \quad . \quad:
$$

LGPK 346

LGPQ 350

FSSK 348

LGSK 344

IGAK 337

F--- 347 


\section{ABBREVIATIONS}

\section{General abbreviations}

aa amino acids

Co-IP

co-immunoprecipitation

$\mathrm{Da}$ dalton

DMEM

Dulbeccos's modified Eagles medium

E.coli Escherichia coli

ECL enhanced chemical luminescence

FCS fetal calf serum

FLIP fluorescence loss in photobleaching

FRAP fluorescence recovery after photobleaching

FRET fluorescence reconance energy transfer

H.s. Homo sapiens

His- hexahistidine tag

IF immunofluorescence

LB Luria-Bertani

MCS multiple cloning site

NCS newborn calf serum

NES nuclear export signal

NLS nuclear localization signal

NPC nuclear pore complex

ORF open reading frame

PAGE polyacrylamide gel electrophoresis

PCR polymerase chain reaction

S.c. Saccharomyces cerevisiae

S.cerevisiae Saccharomyces cerevisiae

$\mathrm{v} / \mathrm{v} \quad$ volume per volume

w/v weight per volume

WB western blot

wt wildtype

$\triangle \mathrm{NLS} \quad$ mutated, unfunctional NLS 


\section{Proteins}

$\begin{array}{ll}\text { BSA } & \text { bovine serum albumine } \\ \text { CFP } & \text { cyan fluorescent protein }\end{array}$

E1 activating enzyme

E2 conjugating enzmye

E3 ligase

GAP GTPase acitvating protein

GFP gree fluorescent protein

imp importin

S1 SUMO1

S2/3 SUMO2/3

SUMO small ubiquitin related modifier

Ub ubiquitin

YFP yellow fluoresencent protein

Chemicals
APS
ammonium persulfate
ATP
adenosine-5'-tripphosphate
DMSO
dimethyl sulfoxide
dNTP
2'-desoxynucleoside-5'-triphosphate
DTT
dithiothreitol
EDTA
ethylenediaminetetraacetic acid
GDP
guanosine diphosphate
GTP
guanosine-5'- triphosphate
HEPES
[4-(2-hydroxyethyl)-1-piperazine]ethanesulfonic acid
IPTG
isopropyl- $\AA-D-1$-thiogalactopyranoside
LMB
leptomycin B
PBS
phosphate buffered saline
PMSF
phenylmethanesulphonylfluoride
SDS
sodium dodecyl sulfate
TEMED
tetramethylethylenediamine
Tris
tris(hydroxymethyl)aminomethane 
TRITC rhodamine isothiocyanate

Triton-X100 4-octylphenol polyethoxylate

Tween-20 polyoxyethylene (20) sorbitan monolaurate

Physical units

$\begin{array}{ll}{ }^{\circ} \mathrm{C} & \text { degree celsius } \\ \mathrm{g} & \text { gram } \\ \mathrm{g} & \text { acceleration of gravity on earth } \\ \mathrm{h} & \text { hour(s) } \\ \mathrm{I} & \text { liter } \\ \mathrm{m} & \text { meter } \\ \mathrm{M} & \text { molar (mol/l) } \\ \mathrm{min} & \text { minute(s) } \\ \mathrm{OD} & \text { optical density } \\ \mathrm{pH} & \text { potential hydrogen } \\ \mathrm{rpm} & \text { rotations per minute } \\ \mathrm{sec} & \text { second(s) }\end{array}$

\section{Prefixes}

$\begin{array}{lll}\mathrm{k} & \text { kilo- } & 10^{3} \\ \mathrm{C} & \text { centi- } & 10^{-2} \\ \mathrm{~m} & \text { mili- } & 10^{-3} \\ \mu & \text { micro- } & 10^{-6} \\ \mathrm{n} & \text { nano- } & 10^{-9} \\ \mathrm{p} & \text { pico- } & 10^{-12}\end{array}$

Code for amino acids
A
Ala
alanine
C
Cys
cysteine
D Asp
aspartate
$\mathrm{E}$
Glu
glutamate 


$\begin{array}{lll}\text { F } & \text { Phe } & \text { phenylalanine } \\ \text { G } & \text { Gly } & \text { glycine } \\ \text { H } & \text { His } & \text { histidine } \\ \text { I } & \text { Iso } & \text { isoleucine } \\ \text { K } & \text { Lys } & \text { lysine } \\ \text { L } & \text { Leu } & \text { leucine } \\ \text { M } & \text { Met } & \text { methionine } \\ \text { N } & \text { Asn } & \text { asparagine } \\ \text { P } & \text { Pro } & \text { proline } \\ \text { Q } & \text { Gln } & \text { glutamine } \\ \text { R } & \text { Arg } & \text { arginine } \\ \text { S } & \text { Ser } & \text { serine } \\ \text { T } & \text { Thr } & \text { threonine } \\ \text { V } & \text { Val } & \text { valine } \\ \text { W } & \text { Trp } & \text { tryptophane } \\ \text { Y } & \text { Tyr } & \text { tyrosine } \\ \text { X } & - & \text { any } \\ \text { Z } & - & \text { apolar residue } \\ \text { Y } & - & \text { bulky hydrophobic residue }\end{array}$




\section{ACKNOWLEDGEMENTS}

Ganz besonders danken möchte ich Frau Prof. Dr. Frauke Melchior für das vielseitige Thema, die herzliche Aufnahme in die Arbeitsgruppe und für Ihren Enthusiasmus mit dem sie mich immer wieder ansteckte wenn meiner mich verließ.

Für die gute Betreuung im Rahmen meines Thesis-Komitees bedanke ich mich bei Herrn Prof. Dr. Ficner und Herrn Prof. Dr. Doenecke. Herrn Prof. Dr. Ficner danke ich des Weiteren für die Übernahme des Korreferates und Herrn Prof. Dr. Doenecke gilt ein ganz besonderes Dankeschön für die stets sehr herzliche Hilfe in allen Angelegenheiten des von ihm geleiteten Graduiertenkollegs 521.

Der Deutschen Forschunggemeinschaft danke ich für die finanzielle Ünterstützung dieser Arbeit im Rahmen des GK 521.

Bedanken möchte ich mich auch bei Herrn Prof. Dr. Elmar Schiebel und Cornelia König für all die Hilfe bei den Hefe Experimenten und der gesamten Gruppe von Dr. Ralph Kehlenbach für die Unterstützung bei den Untersuchungen zum Kerntransport.

Danke an all meine lieben Kollegen der Melchior's und der Kehlenbach's für die unzählige fachliche Unterstützung, die wunderbare Atmosphäre und nicht zu vergessen für das ein oder andere Bier... Ein ganz persönlicher Dank geht an Annette Flotho, Inga Waldmann und Tina Lampe.

Abschließend danke ich Holger und den Menschen denen ich am meisten verdanke: meiner Schwester Anne sowie meinen Eltern Hans und Brigitte - Danke für alles. 


\section{CURRICULUM VITAE}

Personal data

Name: $\quad$ Marie Christine Moutty

Date of birth: $\quad$ 15.12.1981

Place of birth: Heide (Holstein), Germany

Nationality: German

\section{Education}

since 09/2006

Ph.D. student in the laboratory of Prof. Dr. Frauke Melchior, initially Biochemistry I at the University of Göttingen, then ZMBH at the University of Heidelberg

11/2005 - 07/2006 Diploma thesis in the laboratory of Prof. Dr. Gunter Fischer, Max Planck Research Unit for Enyzmology of Protein Folding in Halle (Saale)

10/2001 - 07/2006 Studies of Biochemistry at the University of Halle-Wittenberg in Halle (Saale)

Graduation of the Secondary School, Abitur

$1992-2001$

Secondary School, Gelehrtenschule in Meldorf

Selected presentations

12/2009, poster

09/2009, poster

03/2009, poster

10/2009, poster

08/2007, talk
ASCB in San Diego, California

Retreat of the DKFZ-ZMBH-Alliance in Heidelberg, Germany RUBICON Annual Meetin in Sesimbra, Portugal Retreat of the DKFZ-ZMBH-Alliance in Altleiningen, Germany Minisymposium on Membrane transport/Protein-Protein Interactions in Bad-Münster am Stein - Ebernburg, Germany 
Stipend

09/2006 - 09/2009 Stipend of the DFG within the GK521 on „Protein-ProteinInteractions During Intracellular Transport of Macromolecules"

\section{Publications}

Werner A, Moutty MC, Möller U, Melchior F. Performing in vitro sumoylation reactions using recombinant enzymes; Methods Mol Biol. 2009; 497:187-99.

Edlich F, Maestre-Martínez M, Jarczowski F, Weiwad M, Moutty MC, Malesević M, Jahreis G, Fischer G, Lücke C. A novel calmodulin-Ca2+ target recognition activates the Bcl-2 regulator FKBP38; J Biol Chem. 2007; 282(50):36496-504.

Edlich F, Erdmann F, Jarczowski F, Moutty MC, Weiwad M, Fischer G. The Bcl-2 regulator FKBP38-calmodulin-Ca2+ is inhibited by Hsp90; J Biol Chem. 2007; 282(21):15341-8.

Weiwad M, Edlich F, Kilka S, Erdmann F, Jarczowski F, Dorn M, Moutty MC, Fischer G. Comparative analysis of calcineurin inhibition by complexes of immunosuppressive drugs with human FK506 binding proteins; Biochemistry. 2006; 45(51):15776-84.

Edlich F, Weiwad M, Wildemann D, Jarczowski F, Kilka S, Moutty MC, Jahreis G, Lücke C, Schmidt W, Striggow F, Fischer G. J The specific FKBP38 inhibitor N( $N$ ', $N$ '-dimethylcarboxamidomethyl)cycloheximide has potent neuroprotective and neurotrophic properties in brain ischemia; Biol Chem. 2006; 281(21):14961-70. 